BNL-NUREG -21241-R2

INFORMAL REPORT

\title{
DEVELOPMENT OF SOLUTIONS TO BENCHMARK PIPING PROBLEMS
}

\author{
M. REICH, T. Y. CHANG, AND S. PRACHUKTAM
}

Structural ANALYsis GROUP

BROOKHAVEN NATIONAL LABORATORY

UPTON, NEW YORK 11973

AND

M. HARTZMAN

U.S. Nuclear Regulatory COMmission

WASHINGTON, DC 20555

DECEMBER 1977

DEPARTMENT OF NUCLEAR ENERGY BROOKHAVEN NATIONAL LABORATORY UPTON, NEW YORK 11973 


\section{DISCLAIMER}

This report was prepared as an account of work sponsored by an agency of the United States Government. Neither the United States Government nor any agency Thereof, nor any of their employees, makes any warranty, express or implied, or assumes any legal liability or responsibility for the accuracy, completeness, or usefulness of any information, apparatus, product, or process disclosed, or represents that its use would not infringe privately owned rights. Reference herein to any specific commercial product, process, or service by trade name, trademark, manufacturer, or otherwise does not necessarily constitute or imply its endorsement, recommendation, or favoring by the United States Government or any agency thereof. The views and opinions of authors expressed herein do not necessarily state or reflect those of the United States Government or any agency thereof. 


\section{DISCLAIMER}

Portions of this document may be illegible in electronic image products. Images are produced from the best available original document. 


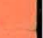

$\sqrt{2}$

NOTICE

This report was prepared as an account of work sponsored by the United States Government. Neither the United States nor the United States Nuclear Regulatory Commission, nor any of their employees, nor any of their contractors, subcontractors, or their employees, makes any warranty, express or implied, or assumes any legal liability or responsibility for the accuracy, completeness or usefulness of any information, apparatus, product or process disclosed, or represents that its use would not infringe privately owned rights. 
DEVELOPMENT OF SOLUTIONS TO BENCHMARK PIPING PROBLEMS*

M. Reich, T. Y. Chang, S. Prachuktam

Structural Analysis Group

Brookhaven National Laboratory

Upton, New York 11973

and

M. Hartzman

U. S. Nuclear Regulatory Commission Washington, DC 20555

- MOTIGE

MN ONLX

December 1977
P0FTIQNS 6F THIS REPORT ARE ILLEGIBLE. It has begen ren raduged from the best available Gavy to permit the broadest possible availability,

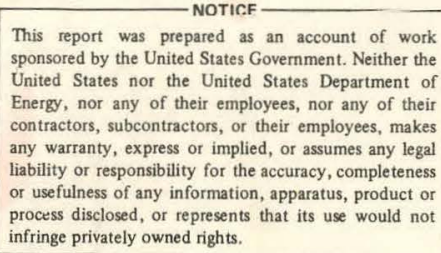

* Work sponsored by the Mechanical Engineering Branch, Division of Systems Safety, United States Nuclear Regulatory Commission. 
This report was prepared as an account of work sponsored by the United States Government. Neither the United States nor the United States Nuclear Regulatory Connission, nor any of their employees, nor any of their contractors, subcontractors, or their employees, makes any warranty, express or implied, or assumes any legal liability or responsibility for the accuracy, completeness or usefulness of any information, apparatus, product or process disclosed, or represents that its use would not infringe privately owned rights. 


\section{$\underline{\text { PAGE }}$}

LIST OF FIGURES

1. Static Analysis

2. Dynamic Analysis

2.1 Mode Superposition Method.

2.2. Direct Integration Method

2.3 Response Spectrum Analysis

Pröblem 1 - Static Response of Hovgaard Bend

Problem 2 - Dynamic Response of Hovgaard Bend

Problem 3 - Dynamic Analysis of Coffee Table

Problem 4 - Static Analysis of Spence's Pipe Work Problem 


\begin{tabular}{|c|c|c|}
\hline FIGURE & TITLE & PAGE \\
\hline $1-1 a$ & Local Coordinate Systems & 3 \\
\hline $1-1 b^{\prime}$ & $\begin{array}{l}\text { Sign Convention for Forces and } \\
\text { Moments }\end{array}$ & 10 \\
\hline $1-2$ & A Hovgaard Bend & 12 \\
\hline $2-1$ & Lumped Mass System for Hovgaard Bend & 25 \\
\hline $2-2$ & $\begin{array}{l}\text { Time History Acceleration of Imperial } \\
\text { Earthquake - El Centro Site - May 18, } \\
1940 \text { - SOOE Direction. }\end{array}$ & 33 \\
\hline $2-3$ & Earthquake spectrum & 35 \\
\hline $3-1 a$ & Isometric Sketch of Coffee Table Problem & 60 \\
\hline $3-16$ & $\begin{array}{l}\text { Finite Element Model and Lumped Mass } \\
\text { Locations for Coffee Table Problem }\end{array}$ & 61 \\
\hline $4-1$ & Isometric Sketch of Spence's Problems & 97 \\
\hline
\end{tabular}


Moments and Forces Acting on the Pipe (Problem 1)

1-2 : Forces and Moments from EPIPE

(Problem 1)

Forces and Moments (Analytical Method)

Pipe Stresses (Problem 1)

$1-5$

Hovgaard Problem Static Analysis (Case A) Node Displacements/Rotations (Case A)

Hovgaard Problem Forces and Moments (Case A)

Hovgaard Problem Pipe Stresses (Case A)

Input Data for Dynamic Response of Hovgaard Bend

Lumped Mass Data Dynamic Response of Hovgaard Bend

Time History. Acceleration for Hovgaard Problem

Acceleration spectrum

Natural Frequencies for Hovgaard Problem (Time History Analysis)

Mode Shapes (Time History Hovgaard)

Mode Shapes (Time History Hovgaard)

Mode Shapes (Time History Hovgaard)

Mode Shape (Time History Hovgaard) 


\section{LIST OF TABLES (CONTINUED)}

TABLE

2-9

$2-10$

$2-11 a$

$2-11 b$

$2-11 c$

$2-11 d$

$2-12$

$2-13$

$2-14$

$2-15$

$2-16$

$3-1$

3-2

$3-3 a$

$3-3$

$3-4$

$3-5$

TITLE

PAGE

Maximum Stress Components (Time History

Hovgaard)

Hovgaard Response Spectrum Analysis Natural

Frequencies

Hovgaard Response Spectrum Analysis Mode

Shapes

Hovgaard Response Spectrum Model

Displacement Components

Hovgaard Response Spectrum Model

Displacement Components

Hovgaard Response Spectrum Model Square Root of Sum of the Square of Displacement

Hovgaard Response Spectrum Model

Force Components

Hovgaard Response. Spectrum Stress

Components

Hovgaard Response Spectrum Displacements and Rotations

Hovgaard Response Spectrum Force Components

Hovgaard Response Spectrum Stress

Components

Input for Coffee Table Problem

Lumped Mass for Coffee Table Problem

Comparison of Natural Frequency Solutions

Coffee Table Time History Analysis Natural

Frequencies

Coffee Table Time History Analysis Mode

Shapes

68

Coffee Table Time History Analysis Maximum Displacements 
Coffee Table Time History Analysis Maximum Force Component

Coffee Table Time History Analysis Maximum Stress Components

Coffee Table Response Spectrum Model

Participation Factors and Earthquake spectrum

Coffee Table Response Spectrum Displacement Components

Coffee Table Response Spectrum Displacement Components

Coffee Table Response Spectrum Displacement Components

Coffee Table Response Spectra Analysis

Force Components

Coffee Table Response Spectra Analyis

Stress Components

Coffee Table step-by-Step Integration

Maximum Displacement

Coffee Table Step-by-step Integration Force Component Maxima

Coffee Table Step-by-step Integration Stress Component Maxima

Forces and Moments for Spence Pipework Problem 
Low Frequency Coffee Table Time History Analysis Nodal Coordinates

Low Frequency Coffee Table Time History Analysis Lump Mass Data

Low Frequency Coffee Table Time History Analysis Natural Frequencies

5-4a Low Frequency Coffee Table Time History Analysis Mode Shape

$5-4 b$

Low Frequency Coffee Table Time History Analysis Mode Shape

Low Frequency Coffee Table Time History Analysis Mode Shape

Low Frequency Coffee Table Time History Analysis Mode Shape

Low Frequency Coffee Table Time History Analysis Mode Shape

Low Frequency Coffee Table Time History Analysis Displacement Maxima

Low Frequency Coffee Table Time History Analysis Force Component Maxima

Low Frequency Coffee Table Time History Analysis Stress Component Maxima

Low Frequency Coffee Table Time History Analysis Modal Participation Factors and Earthquake Response 
Low Frequency Coffee Table Response Spectrum Force Components

$5-9 f$

Low Frequency Coffee Table Response Spectrum Force Components

Low Frequency Coffee Table Response Spectrum Stress Components

Low Frequency Coffee Table Displacement Maxima

Low Frequency Coffee Table Force

Component Maxima

$5-13$

Low Frequency Coffee Table Stress

Component Maxima 


\section{Introduction}

Dynamic structural analysis of piping systems is one of the most extensive engineering efforts required for the safety design of nuclear power plants. Such analysis is normally performed by using computer programs which can handle complex system geometries and various loading conditions, static or dynamic.

Applicants for nuclear power plant licenses are required to provide confirmation of the adequacy of their programs, as prescribed by the guidelines of the Standard Review Plan and Appendix.B, Section III, of 10CFR50.1. Of particular concern to the Mechanical Engineering Branch is the class of programs which are used by the industry to perform dynamic structural analyses of complex piping systems. There are generally large programs based on the finite element method, which consider the structures to be elastic over the entire deformation history and to experience small displacements and rotations (which may, however, contain gaps). Some of these programs are well known, general purpose programs, while others are more of a proprietary nature.

A standard method for verifying computer programs is by means of benchmark problems. By verification we define the assurance that these programs produce correct and valid results; in accordance with the numerical and theoretical methods on which they are based. The verification of these programs can also be considered as their qualification for solving problems of the kind for which they are designed. 
To verify and confirm the quality of these programs, the Mechanical Engineering Branch of the Division of Systeris Safety, in conjunction with the Structural Analysis Group of Brookhaven National Laboratory, has defined a set of suitable benchmark problems and developed the corresponding solutions. The direct objective of these benchmark problems is to provide assurance that programs perform according to the claims of their authors when applied to typical analysis problems encountered in the design of nuclear piping systems.

The purpose of this report is to describe the adopted benchmark problems and their solutions. The problems consist in calculating the static and dynamic response of selected piping structures subjected to a variety of loading conditions. The structures range from simple pipe geometries to a representative full scale primary nuclear piping system, which includes the various components and their supports. These structures are assumed to behave in a linear elastic fashion only, i.e., they experience small deformations and small displacements with no existing gaps, and remain elastic thru their entire response. The solutions were obtained by using the program EPIPE, which is a modification of the widely available program SAP IV. A. brief outline of the theoretical background of this program, and its verification is also included in this report. 


\section{Background}

The EPIPE program is a modified version of the general purpose program SAP $\mathrm{IV}^{4}$, specifically prepared for static and dynamic analysis of the class 1 nuclear piping according to the ASME requirements. ${ }^{5}$ The piping system may consist of straight elements, elbows and tees with spring hangers and anchor restraints. The loads can be either mechanical loads, weights, thermal loads, support movements, earthquakes, or any combination of the aforementioned conditions.

since the elastic piping analysis method is a well established procedure, in depth discussions of the theoretical development will not be included herein. Instead, only a brief outline of the theoretical considerations used in obtaining the static and dynamic solutions to be discussed later with this text, is included in this section.

1. Static Analysis

The static analysis of a piping system is carried out by use of the stiffness matrix method, in which the piping is represented by a network of basic elements (straight and curved beams, and one-dimensional elements) interconnected at the nodes. The formulation of stiffness matrices for straight pipe (beam) and curved pipe may be found in several references such as (6) and (7). For a curved pipe or tee, the flexibility factor and stress intensification factor must 
be considered in the.formulation of the stiffness matrix and in the stress calculations, respectively. These factors are generally functions of pipe geometry and internal pressure. 5,8

From the EPIPE program, the static response of a piping system is obtained in the form of nodal displacements, resultant forces, and stresses at designated locations.

2. Dynamic Analysis

The dynamic response of a piping system is described mathematically by the equations of motion

$$
[M]\{\ddot{u}\}+[c]\{\dot{u}\}+[K]\{u\}=\{R(t)\} \text {. }
$$

Only the lumped mass approximation is allowed. Thus, in equation (1) $M$ represents the diagonal mass matrix of the structure, $\ddot{u}$, the nodal acceleration vector; $\dot{u}$, the nodal velocity vector; $C$, a damping matrix, for which the Ragleigh damping is assumedile, $c=\alpha M+\beta K$, where both $\alpha$ and $e$ are the damping coefficients; $K$, the stiffness.matrix (same as the static problem), and $R(t)$, either a vector of arbitrary time-dependent loads or of effective loads resulting from earthquake motion.

The dynamic analysis may be performed by either the mode superposition method, the direct. integration method, or the response spectrum analysis. All three methods are available in the EPIPE program. 


\subsection{Mode Superposition Method}

In order to employ the mode superposition method, it is first necessary to compute the natural frequencies (or eigenvalues) and corresponding mode shapes of the piping system. A choice of two different solution procedures is available in the EPIPE program to find the eigenvalues: a determinant search technique, or a subspace iteration method. ${ }^{3}$ when the stiffness matrix of the structure can be stored in the computer code as a single block, it is more convenient to use the determinant search technique. However, when the structural stiffness has to be stored in several blocks, it is more efficient to use the subspace iteration method.

In the mode superposition method, the dynamic response of the piping is assumed to be adequately described by p lowest vibration modes. Subsequently, the equations of motion (1) are decomposed into $p$ uncoupled second order differential equations. Each equation is solved by numerical integration and the final response is obtained by superposition of the various contributions of the individual modes.

2.2 Direct Integration Method

The equations of motion (I) can be solved directly by step-by-step numerical integration. From this analysis a complete time-history response of the piping is obtained. 
In the EPIPE program, the wilson $\theta$ method is used, which is considered to be unconditionally stable. The numerical algorithm of this method is outlined in references (3) and (4)。

\subsection{Response spectrum Analysis}

The response spectrum analysis method is often used to determine the dynamic response of a piping system due to earthquake ecitations. It is usually offered as an acceptable substitute for the more complex (and hence more costly) time history analysis.

Similar to the mode superposition method, this method assumes that the response of a structure, can be adequately described by $p$ lowest vibration modes. The maximum displacement corresponding to mode $\mathrm{n}$ is obtained from the procedure outlined in (8). The total response for displacements and stress resultants at each node is calculated as the square root of the sum of the squares of the modal maximum responses. The response spectrum method normally gives higher (or more conservative) values than those obcained from the two other methods. 


\section{Benchmark Problems}

The EPIPE program was applied to the analyses of the following benchmark problems:

1. Static response of Hovgaard bend.

2. Dynamic response of Hovgaard bend.

Case $a, b$, and $c$

3. Dynamic response of a coffee table.

Case $a, b$ and $c$

4. Static analysis of Spence's pipework.

5. Dynamic response of a low frequency coffee table.

The static Hovgaard problem was solved analytically by

Hovgaard for a piping system under temperature load. The benchmark shown here compares this solution with one obtained by. Epipe.

For the dynamic problems, 3 and 5 , the piping systems were assumed to be subjected to ground motion of the Imperial Valley Earthquake, El Centro Site, May, 1940, in the S-E direction. A digitized acceleration time-history was obtained from reference (9) and corresponding to this accelerogram, the spectral acceleration was generated according to the procedure outlined in reference (10). This information was then used as the load input for the response spectrum analysis. The dynamic response for both problems were analyzed by all three methods outlined previously in section II, i.e., : 
Case a - Time-history analysis (mode superposition),

Case b - Response spectrum analysis,

Case c - step-by-step (direct) integration.

The output for the dynamic analysis are presented in the form of:

a) Natural frequencies and vibration modes.

b) Modal participation factors (for case b only).

c) Maximum nodal displacements and their occurrence time.

d) Maximum forces and moments at nodes.

e) Maximum stresses.

It is noted that the maximum forces, moments and stresses are the absolute maximum values.

Finally, the last benchmark solution is for a pipework problem provided to BNL by spence. 11 For this case EPIPE results are compared with those obtained from SPANDLE, a British piping program.

\section{Sign convention.}

Element forces and moments are calculated with respect to the local coordinates defined at the nodes $i$, and $j$ as shown in Fig. 1-1a and 1-1b. 

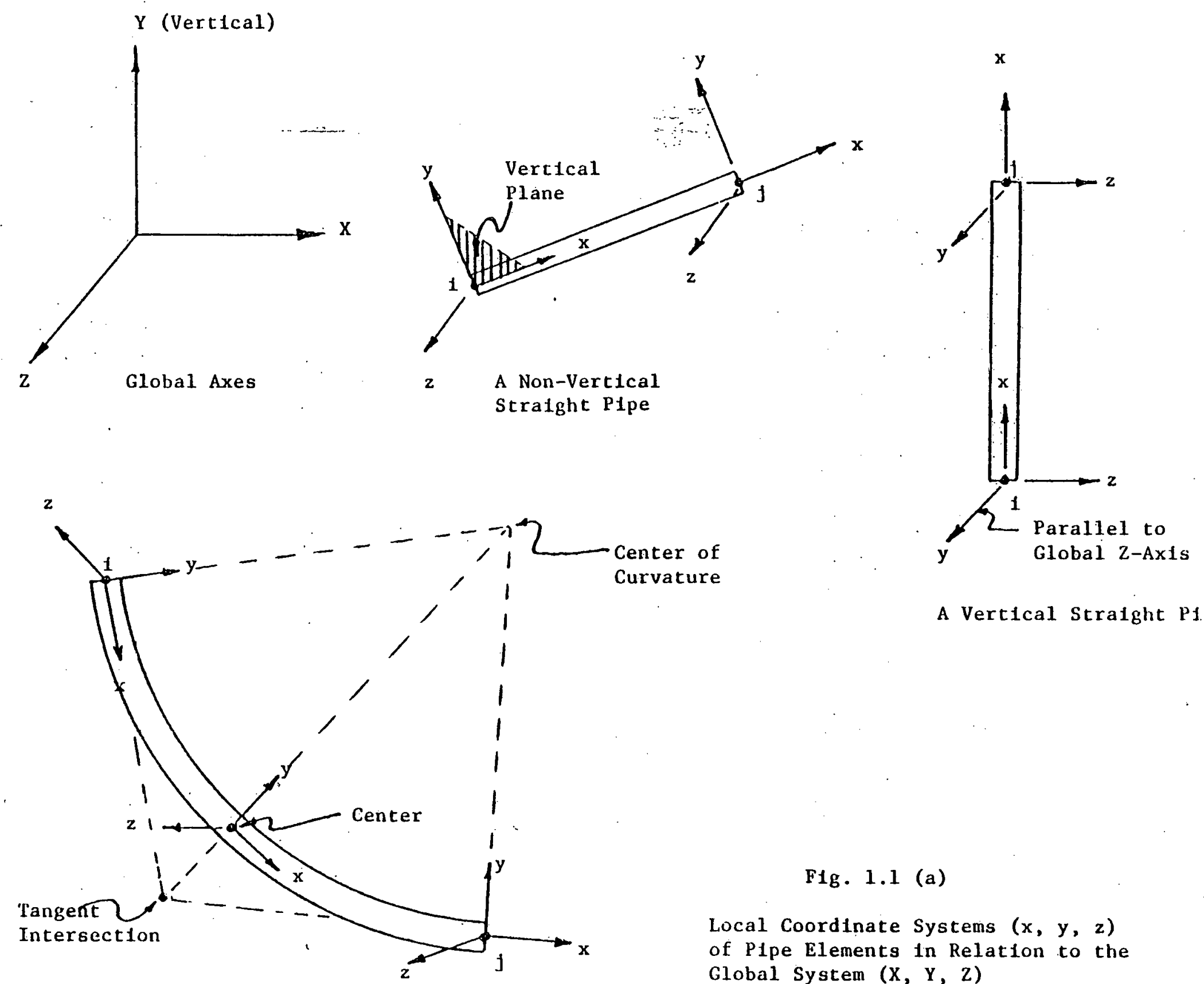

Center of

Curvature

A Vertical Straight Plpe

F1g. 1.1 (a)

Local Coordinate Systems ( $x, y, z)$ of Pipe Elements in Relation to the Global System $(X, Y, Z)$

A Curved Pipe 
Straight Pipe Element

Bend Pipe Element

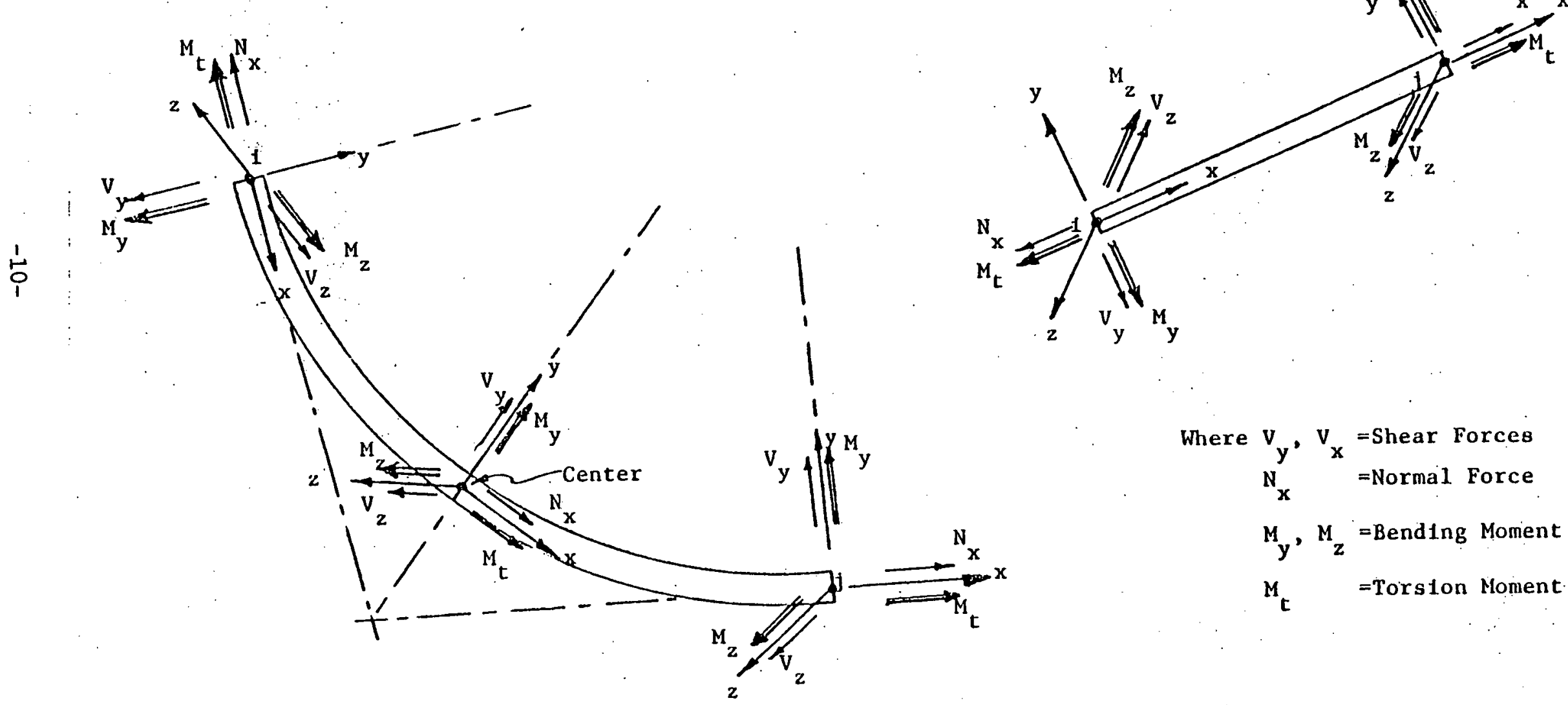

F1g. 1.1 (b)

Sign Convention for Forces and Moments 


\section{Problem.1 - Static Response of Hovgaard Bend}

The response of a three-dimensional three-arm pipe, known as 'Hovgaard bend' subjected to a temperature gradient, has been analyzed. The resultant moments and forces in the pipe were obtained analytically by Hovgaard, 12,13 and numerically by the computer program PIPDYN ${ }^{14}$. A comparison of these solutions is given in the data and tables that follow the problem description.

\section{Problem Description}

i. Geometry - The configuration of the Hovgaard bend, as shown in Fig. 1-2, is comprised of a three-arm pipe in which the arms are at right angles to each other and connected by 90 degree bends. Both ends of the pipe are fixed. The member properties are as follows:

Outside diameter (inches) 7.288

Wall thickness (inches) 0.241

ii. Material data -

Young's modulus (psi) 24.0 E6

Poisson's ratio $\quad 0.3$

Unit weight $\left(\mathrm{lb} /\right.$ inch $\left.^{3}\right) \quad 0.283$

Coefficient of thermal

expansion (in/in/ $\left./{ }^{\circ}\right) \quad 7.1 E-6$

iii. Loading conditions - The piping system is subjected to a uniform temperature of: $850^{\circ} \mathrm{F}$.

"No internal pressure was applied to the system" 


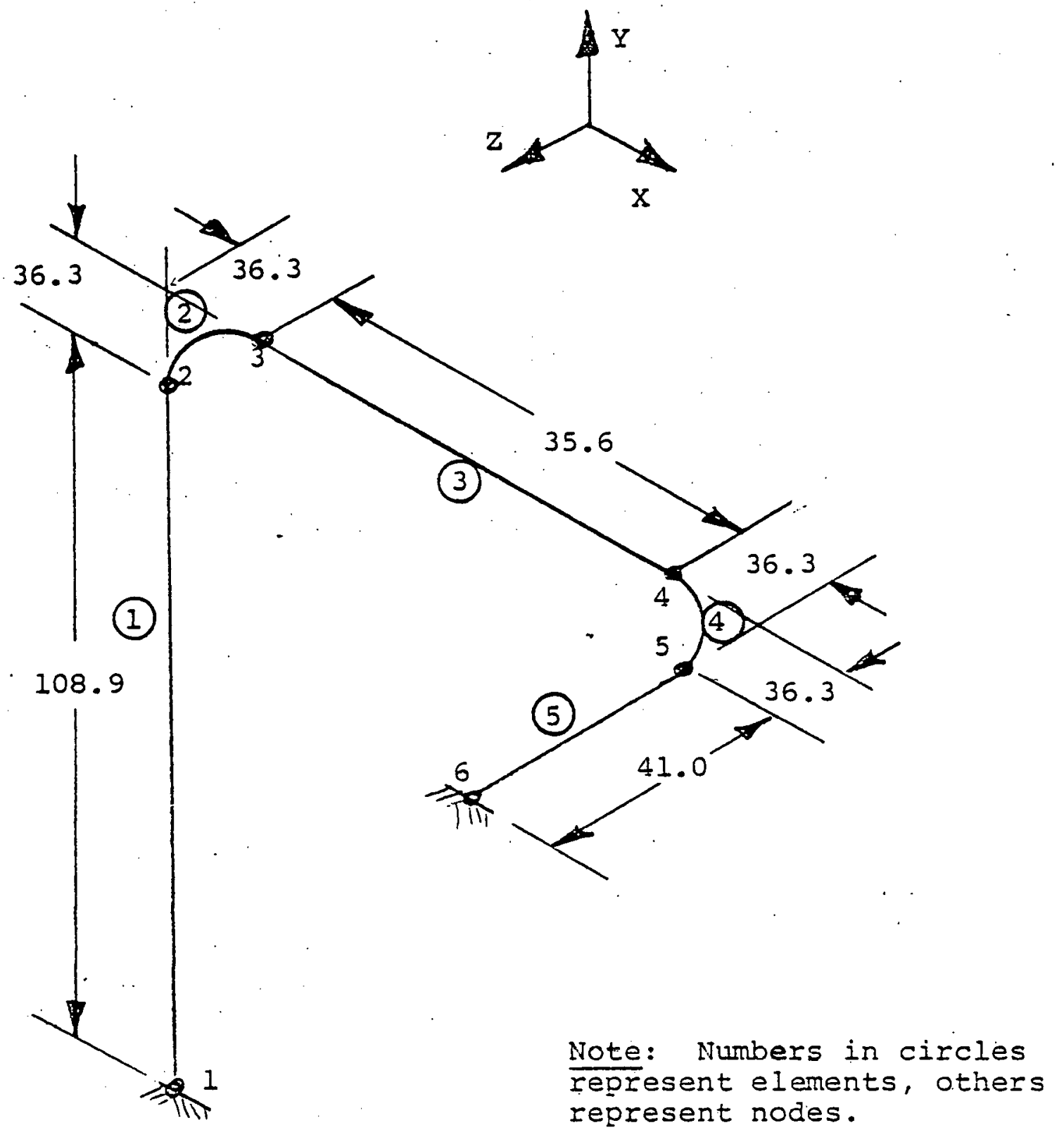

Fig. 1-2

A Hovgaard Bend

(Figure not to scale) 


\section{Analysis Results}

In Table 1-1, the end reactions at ends $A$ and $B$ are compared with those given in references $(12,13,14)$. Note that in the analytical method, the flexibility factor does not account for the effects of internal pressure. Furthermore, it should also be mentioned that the analytical results shown in the tables that follow were obtained from a computerized solution of equations $20,21,22,23,24$ and 25 , given in reference 13.

For the purpose of comparison, Tables $1-2$ and $1-3$ show the resultant forces of each member obtained by "EPIPE' program (Case, a) and by the analytical method, respectively. A comparison of the resulting stresses is shown in Table 1-4. As can be seen from the tables, the differences among the three different solutions are rather small and can be considered to be within an acceptable range.

rable 1-5 to $1-T$ show the computer output of the nodal displacements, resultant forces, and pipe stresses for the case under consideration. 
Table 1-1

Moments and Forces Acting on the Pipe from the Restraints

\begin{tabular}{|c|c|c|c|c|c|c|}
\hline & \multicolumn{2}{|c|}{ EPIPE } & \multicolumn{2}{|c|}{$\begin{array}{c}\text { Analytical Method } \\
{[18]}\end{array}$} & \multicolumn{2}{|c|}{$\begin{array}{l}\text { PIPDYN } \\
{[14]}\end{array}$} \\
\hline & End $A$ & End $B$ & End A & End B & End A & End B \\
\hline${ }_{x}^{M}(i n-1 b)$ & -54876.1 & .92504 .8 & -56040 & $+95295 *$ & -55624.8 & +93732.5 \\
\hline$M_{y}(i n-1 b)$ & $+14336: 0$ & -78555.0 & +14400 & $-80363^{\star}$ & +14494.8 & -79972.8 \\
\hline$M_{z}(i n-1 b)$ & +130165.2 & -62255.6 & +133080 & $-64173 *$ & +132303.6 & -62868 \\
\hline$E_{x}(1 b)$ & -1711.0 & +1711.0 & -1750 & +1750 & -1737.2 & +1737.2 \\
\hline$E_{y}(1 b)$ & -1666.9 & +1666.9 & -1710 & +1710 & -1686.3 & +1686.3 \\
\hline$F_{z}(I b)$ & -628.2 & +628.2 & -640 & +640 & -636.7 & +636.7 \\
\hline
\end{tabular}

* Evaluated from the static equilibrium based on the solutions at End-A from Ref. [18]. 
Table 1-2

Pipes Forces and Moments from 'EPIPE'

\begin{tabular}{|c|c|c|c|c|c|c|c|c|}
\hline Member & Type & Station & Axial & $\begin{array}{l}Y \text {-Axis } \\
\text { Shear }\end{array}$ & $\begin{array}{l}\mathrm{Z}-\mathrm{Axis} \\
\text { Shear }\end{array}$ & $\begin{array}{c}\text { Torsional } \\
\text { Moment }\end{array}$ & $\begin{array}{l}\text { Y-Axis } \\
\text { Moment }\end{array}$ & $\begin{array}{l}\text { z-Axis } \\
\text { Moment }\end{array}$ \\
\hline 1 & Tangent & $\begin{array}{l}\text { End-I } \\
\text { End-J }\end{array}$ & $\begin{array}{l}-1666.9 \\
-1666.9\end{array}$ & $\begin{array}{l}-628.2 \\
-628.2\end{array}$ & $\begin{array}{l}-1711.0 \\
-1711.0\end{array}$ & $\begin{array}{l}14336.0 \\
14336.0\end{array}$ & $\begin{array}{r}130165.2 \\
-56159.2\end{array}$ & $\begin{array}{r}-54876.1 \\
13539.6\end{array}$ \\
\hline 2 & Bend & $\begin{array}{l}\text { End-I } \\
\text { Center }\end{array}$ & $\begin{array}{l}-1666.9 \\
-2388.5\end{array}$ & $\begin{array}{r}-1711.0 \\
-\quad 31.2\end{array}$ & $\begin{array}{l}628.2 \\
628.2\end{array}$ & $\begin{array}{l}14336.0 \\
26390.5\end{array}$ & $\begin{array}{l}13539.6 \\
15562.7\end{array}$ & $\begin{array}{l}56159.2 \\
82353.9\end{array}$ \\
\hline & & End-J & -1711.0 & 1666.9 & 628.2 & 36344.9 & 8469.3 & 57759.6 \\
\hline 3 & Tangent & $\begin{array}{l}\text { End-I } \\
\text { End-J }\end{array}$ & $\begin{array}{l}-1711.0 \\
-1711.0\end{array}$ & $\begin{array}{l}-1666.9 \\
-1666.9\end{array}$ & $\begin{array}{l}-628.2 \\
-628.2\end{array}$ & $\begin{array}{l}36344.9 \\
36344.9\end{array}$ & $\begin{array}{r}8469.3 \\
-30897.6\end{array}$ & $\begin{array}{r}-57759.6 \\
1747.9\end{array}$ \\
\hline 4 & Bend & $\begin{array}{l}\text { End-I } \\
\text { Center }\end{array}$ & $\begin{array}{l}-1711.0 \\
-1654.1\end{array}$ & $\begin{array}{r}-628.2 \\
765.6\end{array}$ & $\begin{array}{l}1666.9 \\
1666.9\end{array}$ & $\begin{array}{l}363.44 .9 \\
44.657 .9\end{array}$ & $\begin{array}{r}1747.9 \\
18321.6\end{array}$ & $\begin{array}{l}30897.6 \\
28832.3\end{array}$ \\
\hline & & End-J & -628.2 & 1711.0 & 1666.9 & 62255.6 & 24162.8 & -8405.3 \\
\hline 5 & Tangent & $\begin{array}{l}\text { End-I } \\
\text { End-J }\end{array}$ & $\begin{array}{l}-628.2 \\
-628.2\end{array}$ & $\begin{array}{l}-1666.9 \\
-1666.9\end{array}$ & $\begin{array}{l}1711.0 \\
1711.0\end{array}$ & $\begin{array}{l}62255.6 \\
62255.6\end{array}$ & $\begin{array}{r}8405.3 \\
78555.0\end{array}$ & $\begin{array}{l}24162.8 \\
92504.8\end{array}$ \\
\hline
\end{tabular}


Table $1-3$

Pipes Forces and Moments (Analytical Method)*

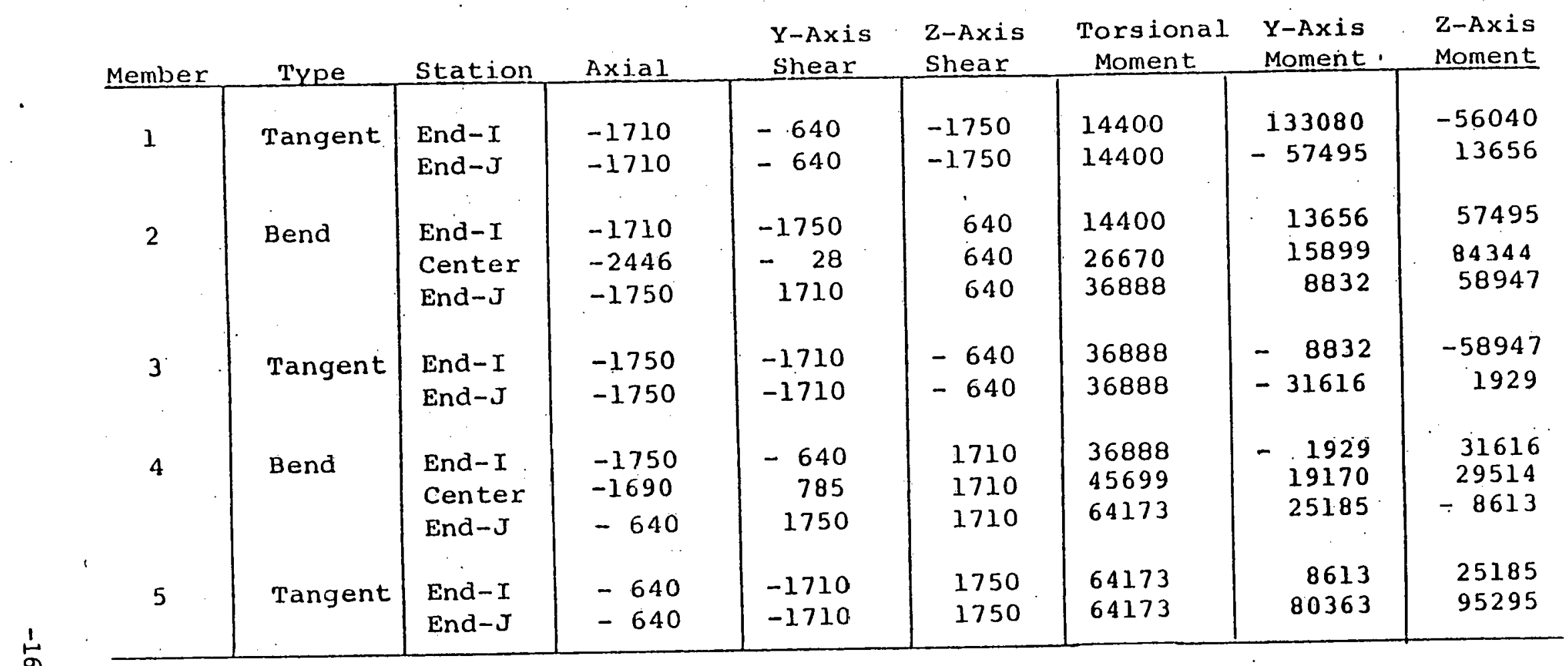

Evaluation based on the solution at End- $\mathrm{h}$ from Ref. 18 and static equilibrium of the system. 
Table 1-4

Pipes Stresses

a) Longitudinal Stress

\begin{tabular}{|c|c|c|c|c|c|c|c|c|}
\hline \multirow[b]{2}{*}{ Member } & \multirow[b]{2}{*}{ Type } & \multirow[b]{2}{*}{ station } & \multicolumn{2}{|c|}{ EPIPE } & \multicolumn{2}{|c|}{$\begin{array}{c}\text { Analtyical } \\
\text { Method }\end{array}$} & \multicolumn{2}{|c|}{ Difference } \\
\hline & & & Tension & Comp. & Tension & Comp. & Tension & Comp: \\
\hline 1 & Targent & $\begin{array}{l}\text { End-I } \\
\text { End-J }\end{array}$ & $\begin{array}{r}15212 \\
6036\end{array}$ & $\begin{array}{l}-15836 \\
-6661\end{array}$ & $\begin{array}{r}15548 \\
6174\end{array}$ & $\begin{array}{l}-16188 \\
-6814\end{array}$ & $\begin{array}{l}2.16 \\
2.24\end{array}$ & $\begin{array}{l}2.17 \\
2.24\end{array}$ \\
\hline 2 & Bend & $\begin{array}{l}\text { End-I } \\
\text { Center } \\
\text { End-J }\end{array}$ & $\begin{array}{r}6903 \\
10021 \\
6971\end{array}$ & $\begin{array}{l}-7528 \\
-10916 \\
-7612\end{array}$ & $\begin{array}{r}7083 \\
10257 \\
7139\end{array}$ & $\begin{array}{l}-7723 \\
-11173 \\
-7795\end{array}$ & $\begin{array}{l}2.54 \\
2.30 \\
2.35\end{array}$ & $\begin{array}{l}2.52 \\
2.30 \\
2.35\end{array}$ \\
\hline 3. & Tangent & $\begin{array}{l}\text { End-I } \\
\text { End-J }\end{array}$ & $\begin{array}{l}6095 \\
3080\end{array}$ & $\begin{array}{l}-\quad 6736 \\
-\quad 3722\end{array}$ & $\begin{array}{l}6222 \\
3153\end{array}$ & $\begin{array}{l}-6878 \\
-3809\end{array}$ & $\begin{array}{l}2.04 \\
2.31\end{array}$ & $\begin{array}{l}2.06 \\
2.28\end{array}$ \\
\hline 4 & Bend & $\begin{array}{l}\text { End-I } \\
\text { Center } \\
\text { End-J }\end{array}$ & $\begin{array}{l}3548 \\
3957 \\
3078\end{array}$ & $\begin{array}{l}-4186 \\
-4577 \\
-3313\end{array}$ & $\begin{array}{l}3640 \\
4093 \\
3215\end{array}$ & $\begin{array}{l}-4296 \\
-\quad 4725 \\
-\quad 3455\end{array}$ & $\begin{array}{l}2.53 \\
3.32 \\
4.26\end{array}$ & $\begin{array}{l}2.56 \\
3.13 \\
4.11\end{array}$ \\
\hline 5 & Tangent & $\begin{array}{l}\text { End-I } \\
\text { End-J }\end{array}$ & $\begin{array}{r}2694 \\
13219\end{array}$ & $\begin{array}{l}-2929 \\
-13455\end{array}$ & $\begin{array}{r}2805 \\
13579\end{array}$ & $\begin{array}{l}-3045 \\
-13819\end{array}$ & $\begin{array}{l}3.96 \\
2.65\end{array}$ & $\begin{array}{l}3.81 \\
2.63\end{array}$ \\
\hline
\end{tabular}


Table 1-4 (cont'd)

Pipes Stresses

b) Circumferential Stress

\begin{tabular}{|c|c|c|c|c|c|c|c|c|}
\hline \multirow[b]{2}{*}{ Member } & \multirow[b]{2}{*}{ Type } & \multirow[b]{2}{*}{ Station } & \multicolumn{2}{|c|}{ EPIPE' } & \multicolumn{2}{|c|}{$\begin{array}{l}\text { Analytical } \\
\text { Method } \\
\end{array}$} & \multicolumn{2}{|c|}{ z Difference } \\
\hline & & & Tension & Comp. & Tension & Comp. & Tension & Comp. \\
\hline 1 & Tangent & $\begin{array}{l}\text { End-I } \\
\text { End-J }\end{array}$ & $\begin{array}{l}0 \\
0\end{array}$ & $\begin{array}{l}0 \\
0\end{array}$ & $\begin{array}{l}0 \\
0\end{array}$ & $\begin{array}{l}0 \\
0\end{array}$ & $\begin{array}{l}0 \\
0\end{array}$ & $\begin{array}{l}0 \\
0\end{array}$ \\
\hline 2 & Bend & $\begin{array}{l}\text { End-I } \\
\text { Center } \\
\text { End-J. }\end{array}$ & $\begin{array}{l}10565 \\
15492 \\
10866\end{array}$ & $\begin{array}{l}-11935 \\
-17502 \\
-12275\end{array}$ & $\begin{array}{l}10453 \\
15334 \\
10716\end{array}$ & $\begin{array}{r}11809 \\
-17325 \\
-12108\end{array}$ & $\begin{array}{l}1.07 \\
1.03 \\
1.40\end{array}$ & $\begin{array}{l}1.15 \\
1.02 \\
1.38\end{array}$ \\
\hline 3 & Tangent & $\begin{array}{l}\text { End-I } \\
\text { End-J }\end{array}$ & $\begin{array}{l}0 \\
0\end{array}$ & $\begin{array}{l}0 \\
0\end{array}$ & $\begin{array}{l}0 \\
0\end{array}$ & $\begin{array}{l}0 \\
0\end{array}$ & $\begin{array}{l}0 \\
0\end{array}$ & $\begin{array}{l}0 \\
0\end{array}$ \\
\hline 4 & Bend & $\begin{array}{l}\text { End-I } \\
\text { Center } \\
\text { End-J }\end{array}$ & $\begin{array}{l}5812 \\
5424 \\
1581\end{array}$ & $\begin{array}{l}-6566 \\
-6127 \\
-\quad 1786\end{array}$ & $\begin{array}{l}5748 \\
5366 \\
1565\end{array}$ & $\begin{array}{r}-6494 \\
-\quad 6062 \\
-.1769\end{array}$ & $\begin{array}{l}1.11 \\
1.08 \\
1.02\end{array}$ & $\begin{array}{l}1.11 \\
1.07 \\
1.00\end{array}$ \\
\hline 5 & Tangent & $\begin{array}{l}\text { End-I } \\
\text { End-J }\end{array}$ & $\begin{array}{l}0 \\
0\end{array}$ & $\begin{array}{l}0 \\
0\end{array}$ & $\begin{array}{l}0 \\
0 .\end{array}$ & $\begin{array}{l}0 \\
0\end{array}$ & $\begin{array}{l}0 \\
0\end{array}$ & $\begin{array}{l}0 \\
0\end{array}$ \\
\hline
\end{tabular}


Table 1-4 (cont'd)

Pipes Stresses

c) Shear Stress

\begin{tabular}{|c|c|c|c|c|c|c|c|c|}
\hline \multirow[b]{2}{*}{ Member } & \multirow[b]{2}{*}{ Type } & \multirow[b]{2}{*}{ Station } & \multicolumn{2}{|c|}{ EPIPE } & \multicolumn{2}{|c|}{$\begin{array}{c}\text { Analytical } \\
\text { Method }\end{array}$} & \multicolumn{2}{|c|}{ \& Difference } \\
\hline & & & Max & Min & $\operatorname{Max}$ & Min & $\operatorname{Max}$ & $\operatorname{Min}$ \\
\hline 1 & Tangent & $\begin{array}{l}\text { End-I } \\
\text { End-J }\end{array}$ & $\begin{array}{l}1129 \\
1129\end{array}$ & $\begin{array}{l}446 \\
446\end{array}$ & $\begin{array}{l}1141 \\
1141\end{array}$ & $\begin{array}{l}443 \\
443\end{array}$ & $\begin{array}{l}1.05 \\
1.05\end{array}$ & $\begin{array}{l}0.70 \\
0.70\end{array}$ \\
\hline 2 & Bend & $\begin{array}{l}\text { End-I } \\
\text { Center } \\
\text { End-J }\end{array}$ & $\begin{array}{l}1129 \\
1568 \\
2331\end{array}$ & $\begin{array}{r}446 \\
1332 \\
1663\end{array}$ & $\begin{array}{l}1141 \\
1585 \\
2369\end{array}$ & $\begin{array}{r}443 \\
1545 \\
1685\end{array}$ & $\begin{array}{l}1.05 \\
1.07 \\
1.60\end{array}$ & $\begin{array}{l}0.70 \\
1.00 \\
1.31\end{array}$ \\
\hline 3 & Tangent & $\begin{array}{l}\text { End-I } \\
\text { End-J }\end{array}$ & $\begin{array}{l}2331 \\
2331\end{array}$ & $\begin{array}{l}1663 \\
1663\end{array}$ & $\begin{array}{l}2369 \\
2369\end{array}$ & $\begin{array}{l}1685 \\
1685\end{array}$ & $\begin{array}{l}1.60 \\
1.60\end{array}$ & $\begin{array}{l}1.31 \\
1.31\end{array}$ \\
\hline 4 & Bend & $\begin{array}{l}\text { End-I } \\
\text { Center } \\
\text { End-J }\end{array}$ & $\begin{array}{l}2331 \\
2798 \\
3869\end{array}$ & $\begin{array}{l}1663 \\
2110 \\
2973\end{array}$ & $\begin{array}{l}2369 \\
2863 \\
3984\end{array}$ & $\begin{array}{l}1685 \\
2159 \\
3068\end{array}$ & $\begin{array}{l}1.60 \\
2.27 \\
2.88\end{array}$ & $\begin{array}{l}1.31 \\
2.26 \\
3.10\end{array}$ \\
\hline 5 & Tangent & $\begin{array}{l}\text { End-I } \\
\text { End-J }\end{array}$ & $\begin{array}{l}3869 \\
3869\end{array}$ & $\begin{array}{r}2973 \\
2973\end{array}$ & $\begin{array}{l}3984 \\
3984\end{array}$ & $\begin{array}{l}3068 \\
3068\end{array}$ & $\begin{array}{l}2.88 \\
2.88\end{array}$ & $\begin{array}{l}3.10 \\
3.10\end{array}$ \\
\hline
\end{tabular}


Table 1-4

Pipes Stresses (cont'd)

\begin{tabular}{|c|c|c|c|c|c|c|c|c|}
\hline & & & Longituc & nal Stress & Circumfe & ial & Sh & Stress \\
\hline Member & Type & Station & EPIPE & $\begin{array}{l}\text { Analytical } \\
\text { Method }\end{array}$ & EPIPE & $\begin{array}{c}\text { Analytical } \\
\text { Method }\end{array}$ & EPIPE & $\begin{array}{c}\text { Analytical } \\
\text { Method }\end{array}$ \\
\hline 1 & Tangent & $\begin{array}{l}\text { End-I } \\
\text { End-J }\end{array}$ & $\begin{array}{r}17986.3 \\
9385.1\end{array}$ & $\begin{array}{r}17953.4 \\
8885.9\end{array}$ & $\begin{array}{l}5848.1 \\
5848.1\end{array}$ & $\begin{array}{l}5848.1 \\
5848.1\end{array}$ & $\begin{array}{l}1077.1 \\
1077.1\end{array}$ & $\begin{array}{l}1115.1 \\
1115.1\end{array}$ \\
\hline 2 & Bend & $\begin{array}{l}\text { End-I } \\
\text { Center } \\
\text { End-J }\end{array}$ & $\begin{array}{r}9699.4 \\
12667.3 \\
9817.4\end{array}$ & $\begin{array}{r}8812.3 \\
11574.3 \\
8969.3\end{array}$ & $\begin{array}{l}18657.4 \\
24261.5 \\
18880.2\end{array}$ & $\begin{array}{l}16308.5 \\
21175.6 \\
16572.7\end{array}$ & $\begin{array}{l}1077.1 \\
1534.0 \\
2373.0\end{array}$ & $\begin{array}{l}1115.1 \\
1536.5 \\
2303.6\end{array}$ \\
\hline 3 & Tangent & $\begin{array}{l}\text { End-I } \\
\text { End-J }\end{array}$ & $\begin{array}{l}9416.9 \\
6194.2\end{array}$ & $\begin{array}{l}8932.7 \\
5947.4\end{array}$ & $\begin{array}{l}5848.1 \\
5848.1\end{array}$ & $\begin{array}{l}5848.1 \\
5848.1\end{array}$ & $\begin{array}{l}2373.0 \\
2373.0\end{array}$ & $\begin{array}{l}2303.6 \\
2303.6\end{array}$ \\
\hline 4 & Bend & $\begin{array}{l}\text { End-I } \\
\text { Center } \\
\text { End-J }\end{array}$ & $\begin{array}{l}6455.9 \\
6224.0 \\
5760.1\end{array}$ & $\begin{array}{l}6004.0 \\
6025.2 \\
5499.4\end{array}$ & $\begin{array}{r}12820.5 \\
12382.7 \\
4369.0\end{array}$ & $\begin{array}{r}15583.3 \\
11600.0 \\
4045.6\end{array}$ & $\begin{array}{l}2373.0 \\
2768.7 \\
3844.8\end{array}$ & $\begin{array}{l}2310.6 \\
2749.0 \\
3827.4\end{array}$ \\
\hline 5 & Tangent & $\begin{array}{l}\text { End-I } \\
\text { End-J }\end{array}$ & $\begin{array}{r}5663.4 \\
16308.7\end{array}$ & $\begin{array}{r}5613.4 \\
16047.4\end{array}$ & $\begin{array}{l}5848.1 \\
5848.1\end{array}$ & $\begin{array}{l}5848.1 \\
5848.1\end{array}$ & $\begin{array}{l}3844.8 \\
3844.8\end{array}$ & $\begin{array}{l}3827.4 \\
3827.4\end{array}$ \\
\hline .. & & & & & & & & \\
\hline
\end{tabular}




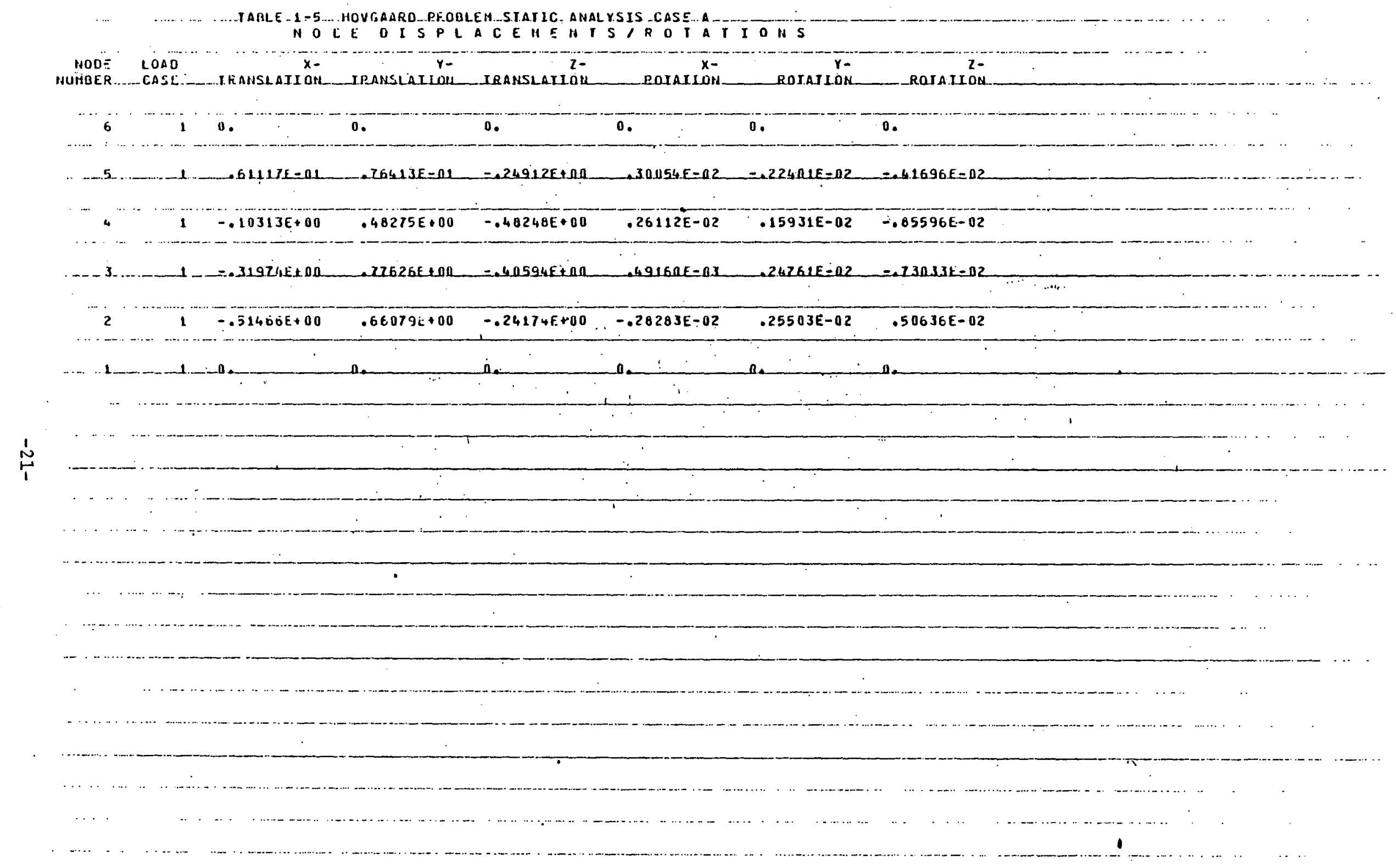


T. TARLE_ I-E HOVGAARO_PEORLEII S.TATIC AHALYSIS CASE A F O F C $S$ A HD HOHENTS

\begin{tabular}{|c|c|c|c|c|c|c|c|c|c|}
\hline $\begin{array}{l}\text { ELEMENI } \\
\text { WUMEEF }\end{array}$ & $\begin{array}{l}\text { BLEMENI } \\
\text { IYPE }\end{array}$ & $\begin{array}{r}\text { LOAO } \\
\text { LASE }\end{array}$ & STATION & $\begin{array}{r}A \times I A L \\
\text { EORCE }\end{array}$ & $\begin{array}{r}Y \text { Y-AXIS } \\
\text { SUEAR }\end{array}$ & $\begin{array}{r}2-A \times I S \\
\text { SHEAR }\end{array}$ & $\begin{array}{r}\text { TORSIONAL } \\
\text { MOMENL }\end{array}$ & $\begin{array}{l}\text { Y-AXIS } \\
\text { MOMEHL }\end{array}$ & $\begin{array}{l}Z \text {-AXIS } \\
\text { MOMENI }\end{array}$ \\
\hline 1 & AHCENT. & & $\begin{array}{l}\because N O=I \\
=N O-J\end{array}$ & $\begin{array}{l}-1 \in C E, 8 Z 8 \\
-16 \in E, 878\end{array}$ & $\begin{array}{l}-528.243 \\
-628.243\end{array}$ & $\begin{array}{l}=171.0 .968 \\
-1710.966\end{array}$ & $\begin{array}{r}14335.95 \\
14335.95\end{array}$ & $\begin{array}{r}130165.22 \\
-56159: 15\end{array}$ & $\begin{aligned}= & 54876.09 \\
& 13539.63\end{aligned}$ \\
\hline 2 & AE & 1 & $\begin{array}{r}\text { ENDI-1 } \\
\text { CENLEE } \\
\text { ENO-J }\end{array}$ & $\begin{array}{l}-166 E .878 \\
=2360.497 \\
-1710.960\end{array}$ & $\begin{array}{r}-1710.968 \\
=31.126 \\
1666.878\end{array}$ & $\begin{array}{r}628.243 \\
-628.243 \\
628.243\end{array}$ & $\begin{array}{r}14335.95 \\
2639.0 .51 \\
36344.06\end{array}$ & $\begin{array}{r}13539.63 \\
15.562 .65 \\
0469.29\end{array}$ & $\begin{array}{l}56159.15 \\
8235.3 .94 \\
57759.62\end{array}$ \\
\hline 3 & rAISGE & 1 & $\begin{array}{r}E H O-I \\
\text { END=J }\end{array}$ & $\begin{array}{l}-1710.960 \\
=1710.968\end{array}$ & $\begin{array}{l}-1666.878 \\
=1666.078\end{array}$ & $\begin{array}{l}-628.243 \\
=628.243 \\
\end{array}$ & $\begin{array}{r}36344.80 \\
36344.86\end{array}$ & $\begin{array}{r}-0469.29 \\
-308.97 .058\end{array}$ & $\begin{array}{r}-57759.62 \\
-1747.91\end{array}$ \\
\hline$\cdots$ & - BEHO & & $\begin{array}{l}\text { EUD }=1 \\
\text { CENTER } \\
\text { ENO }=J\end{array}$ & $\begin{array}{r}-1710.948 \\
-1654.072 \\
-6.28 .243\end{array}$ & $\begin{array}{r}=028.243 \\
765.602 \\
1210.968\end{array}$ & $\begin{array}{r}1666.028 \\
1666.878 \\
1666.878\end{array}$ & $\begin{array}{r}36344.86 \\
44657.94 \\
-62255.52\end{array}$ & $\begin{array}{r}174.919 \\
18321.64 \\
24162.79\end{array}$ & $\begin{array}{r}30892.58 \\
28832.27 \\
=84.05 .30\end{array}$ \\
\hline & TANCENT & -1 & $\begin{array}{l}F N D=I \\
E N D-J\end{array}$ & $\begin{array}{l}-628.243 \\
-628.243\end{array}$ & $\begin{array}{l}-1666.878 \\
-1666.878\end{array}$ & $\begin{array}{l}1710.968 \\
1710.968\end{array}$ & $\begin{array}{r}62255.57 \\
62255.57\end{array}$ & $\begin{array}{r}8405.30 \\
78554.97\end{array}$ & $\begin{array}{l}24162.79 \\
92504.77\end{array}$ \\
\hline
\end{tabular}


IBRLE $1-1$ HUVOAARU PAORLEH SIATIC ANALYSIS CASE A

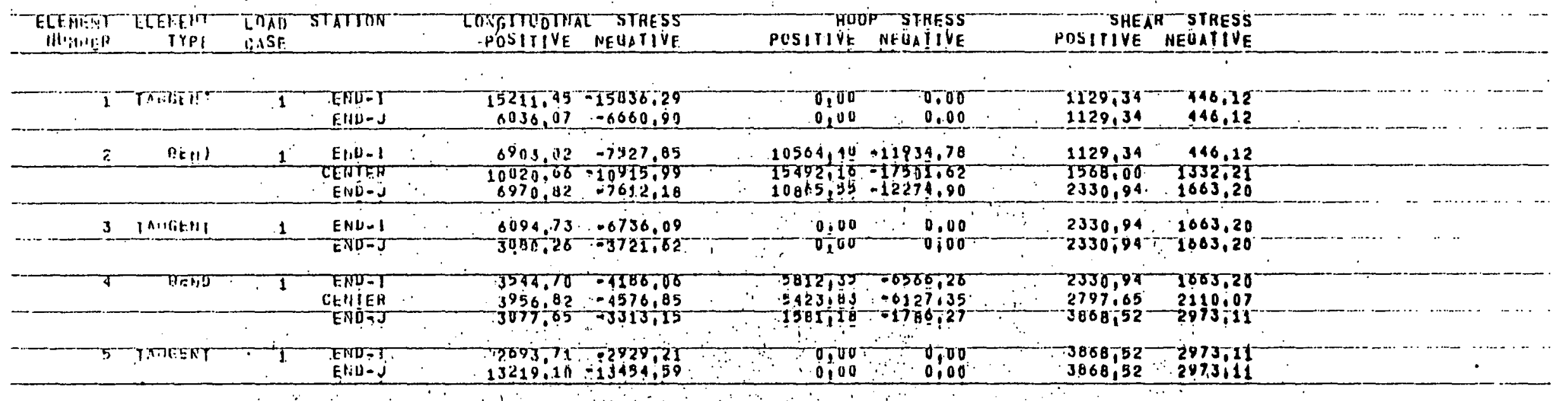

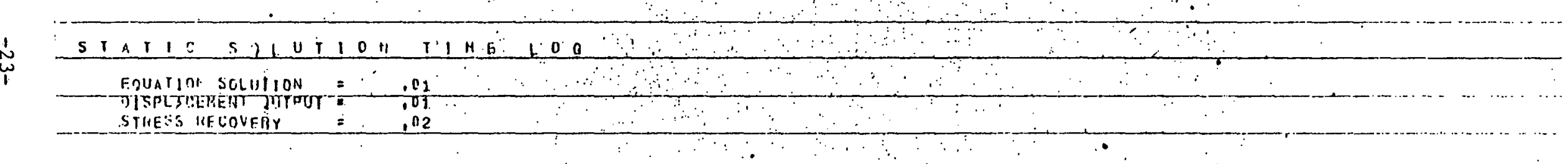

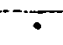




\section{Problem 2 - Dynamic Response of Hovgaard Bend}

The dynamic response of the same piping configuration as Problem $I$ has been analyzed. The piping system is assumed subjected to the $1940 \mathrm{El}$ Centro earthquake. Three different methods of analyses are employed, i.e.:

Case a - Time-history analysis using mode superposition.

Case b-Response spectrum analysis.

Case c - Direct step-by-step integrating method.

The answers gotten from the three different methods of solution are quite close. As expected, the results obtained from the response spectrum analysis are higher than those gotten from the other two methods.

\section{Problem Description}

i. Geometry - The piping geometry is the same as the one discussed previously in Problem 1. Table 2-1 shows the input nodal coordinates. In this analysis, the lumped mass method is used; each member is lumped at three points: one-half of the mass at the center points and one-fourth of the mass at both ends. The proportion of the masses at each point are as shown in Fig. 2-1. Table 2-2 shows the input masses applied the various individual at nodal points.

ii. Material data - same as given in Problem 1. 

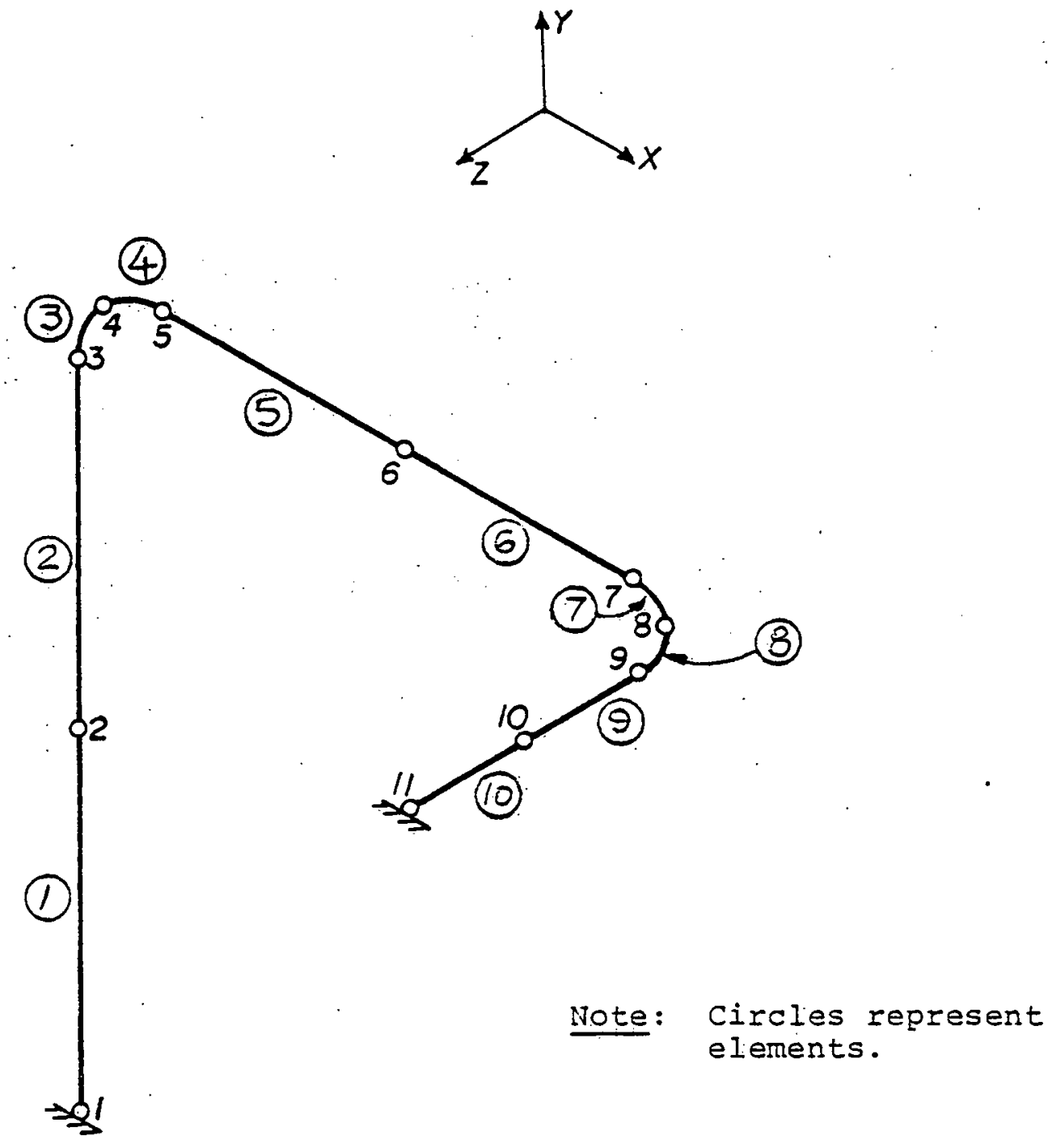

Fig. 2-1

Lumped Mass System for Hovgaard Bend 
IAfI I: ?-1 Input data for Dynamic Response of Hovgaard Bend

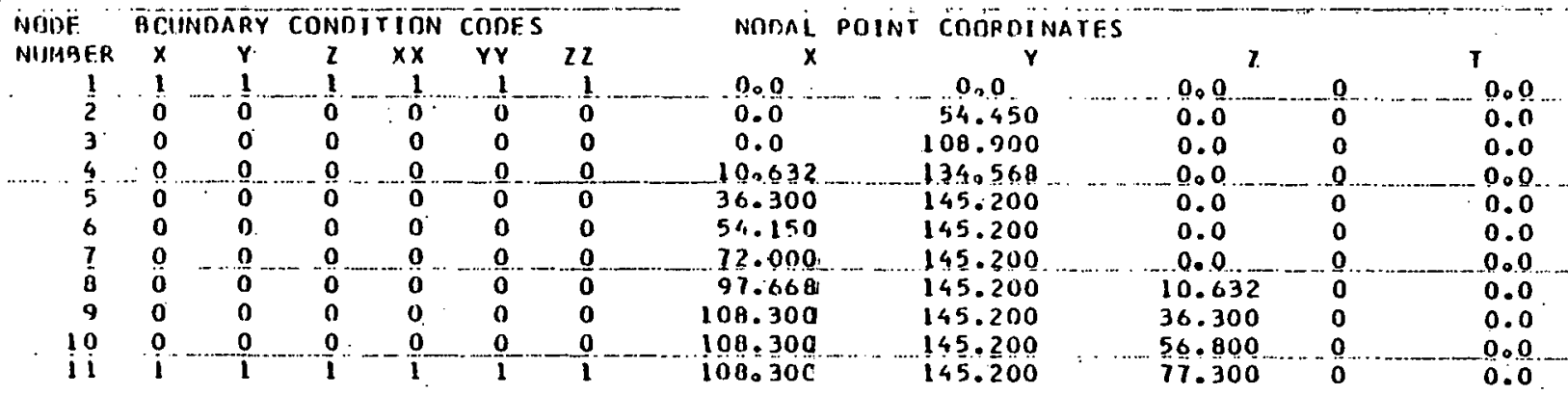




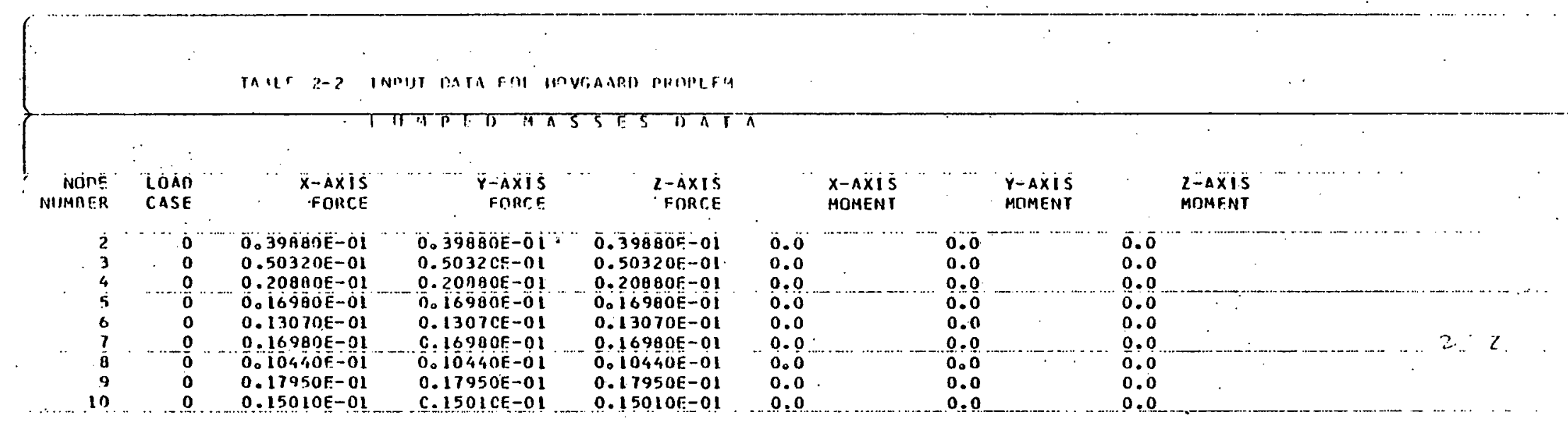


iii. Loading conditions - The piping system is subjected to the ground motion of the Imperial Valley Earthquake, El Centro site, May 1940, in S-E direction. The ground acceleration of the piping system is assumed in the $\mathrm{x}$-direction. Table $2-3$ shows the data of the accelerograph up to 16 seconds at equal intervals of 0.02 seconds. The corresponding accelerograph is plotted in Fig. 2-2.

The same acceleration time-history is used to calculate the spectral acceleration used for the response spectrum analysis. The spectral acceleration resulting from the time history is Iisted in Table 2-4. These values are shown as the dashed lines in Fig. 2-3. The dark lines in Fig. 2-3 show the modified spectrum as per NRC Reg. Guide 1.122. The input values shown in Table 2-10 correspond to those of the modified spectrum.

\section{Analyses Results}

The computer outputs for all three analyses are included in the tables that follow. Tables $2-5$ to $2-9$ give the results for the time-history analysis, including the natural frequencies of the first 5 modes, their associated mode shapes, the maximum displacements with the time of their occurrence, the maximum forces and moments, and the maximum stress components.

Results from the response spectrum analysis are given in Tables 2-10 to 2-13, where the natural frequencies, the mode. shapes, the modal participation factors, and the square root of the sum of the squares of the modal forces and the stresses are shown. Finally, tables 2-14 to 2-16 show the displacement, 
forces and stress results obtained from the step-by-step integration method. The solutions obtained from all three methods appear to be analogous except as noted previously, the response spectrum analysis gives generally higher resultant forces and stresses than those of the other two methods. It is to be noted that the outputs shown in the tables are maximum values over the specified time span. For some computer methods these values are dependent on the integration time step size as well as the print time step size. A complete discussion of this topic can be found in Appencix D of reference 18. For this particular analysis the time span was 16 seconds. with 800 integration steps of 0.02 seconds each. The printout time was consistent with the integration step time, i.e., every integration step was printed. 


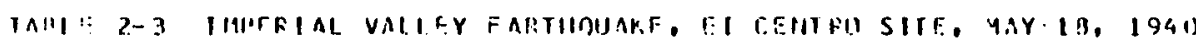

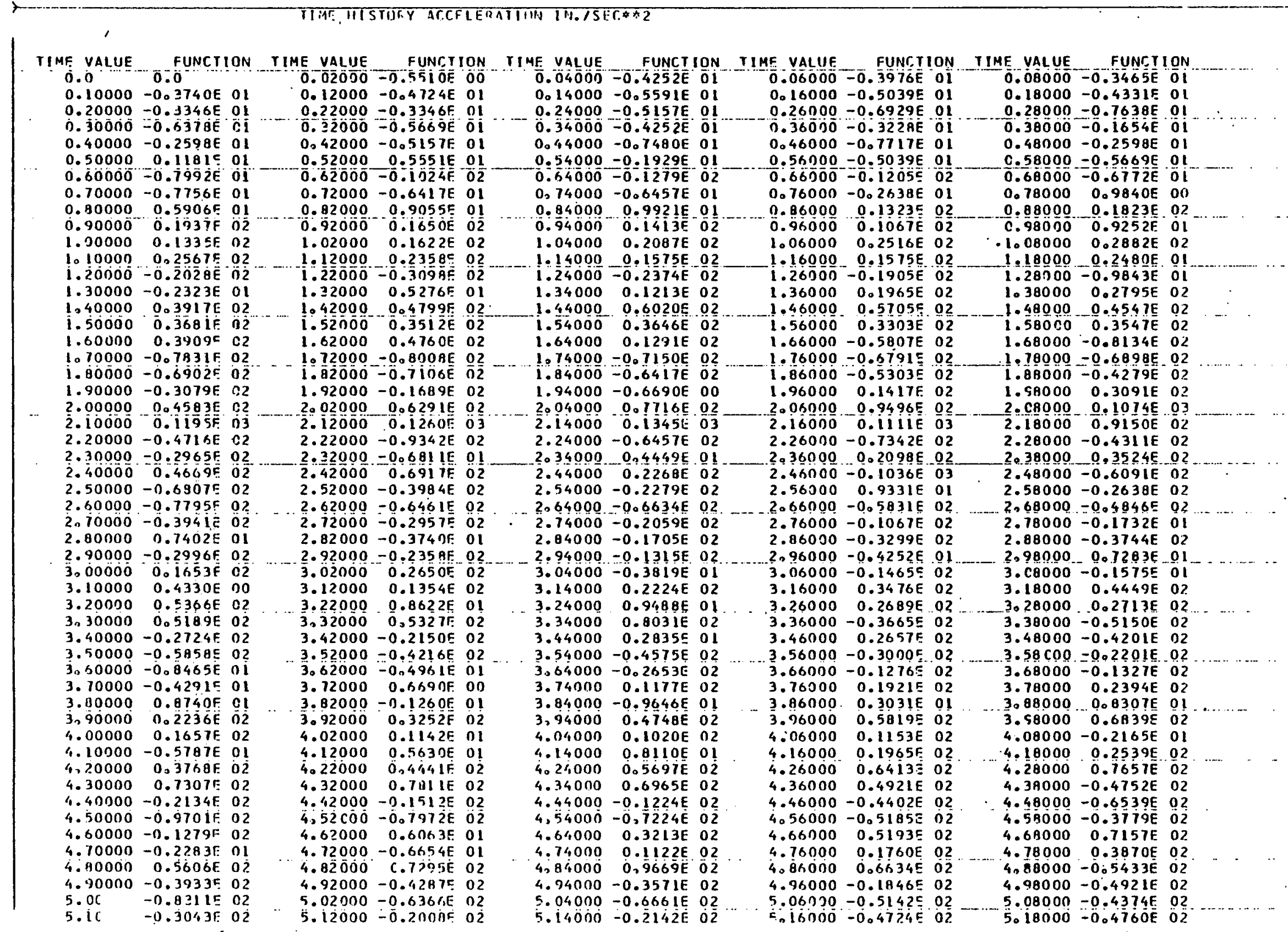




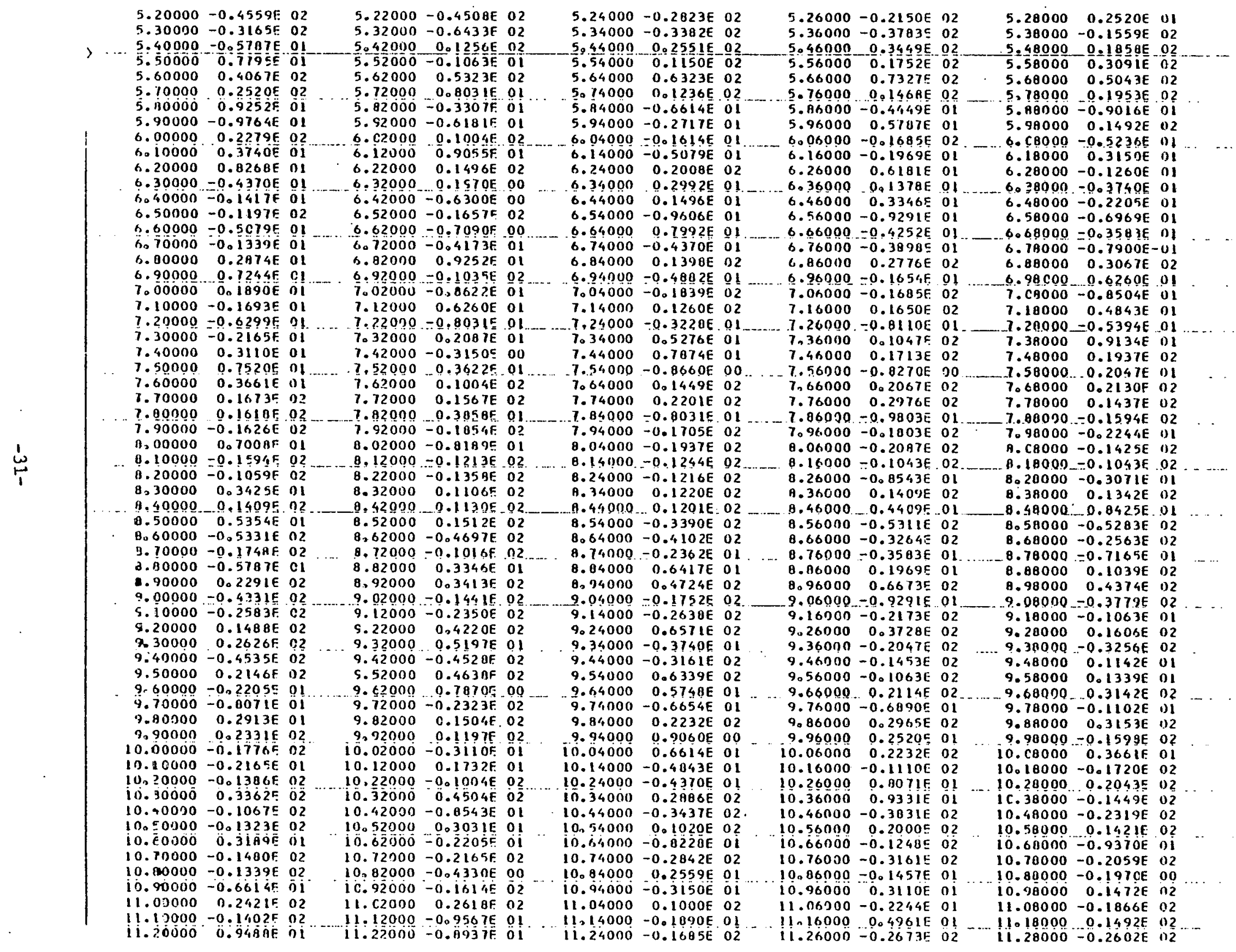


$11.30000-0.2323 E 0.2$ $11.40000-0.2130 E 02$ $11.50000-0.3031 E .02$

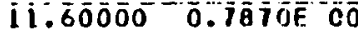
$11.70000 \quad 0.3673 F \quad 02$ $11.00000 \quad 0.5252 \mathrm{E} 02$

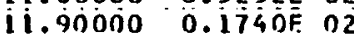
$12.00000-0.3974,02$ 12. $10000-0.2925 \mathrm{~F}$ i $2.20000-0.3437 \mathrm{TE}$ $12.30000 \quad 0.8465 F$ O 12.40000 0.1909E O? i 2.50000 0.7835E 12.60000 C.9409F O1 12. $70000-0.7480 E 00$ 12. B0000 =0.940 O 12.90000 0.2016F 12.90000 0.2016E 02 13,00000 . 0.1079E D2 13.10000 0.323CE 0 $13.20000-0.4843 E 0$ $13030000=0.1063 \mathrm{E} \mathrm{O2}$ $13.40000=0.3268$ E 0 $13.50000-0.3134 \mathrm{E} 02$ $\begin{array}{lll}13.60000 & -0.9882 E & 01 \\ 13.70000 & -0.2630 E & 01\end{array}$ $13.80000 \quad 0.3768 E$ O2 $13.90000-0.3177$ E 02 14.00000 0.1220E OI $14.10000-0.1409502$ 14.20000 0.24896 14.20000 .0248 . 14,30000 0.2957 0 $14.50000-0.7283 \mathrm{E} \mathrm{Cl}$ $14.50000-0.7283 \mathrm{E}$
14.60000
14.000 $14.70000-0.7008 \mathrm{~F} 01$ $14.70000-0.7008 \mathrm{O}$ OI 14.80000 0.1720E 02 $14090000-0.3189 E$ OI $15.00000-0.5567 E$ OI $15.10000 \quad 0.2449602$

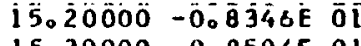
$15.30000-0.8504 E 01$ $15.40000 .0 .6693 \mathrm{E} 01$ i5.50000 $0.23660^{\circ} 02$ 15.50000 0.2571E 02

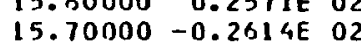
I $5.80000-0.5039 E$ O $15.80000-0.5039 \mathrm{E}$
$15.90000-0.4720 \mathrm{~F}$ $15.90000-0.4720 \mathrm{~F}$
$16.00000-0.1268 \mathrm{~F}$
$11.32000-0.202$ OF 02 $11.42000-0.2472 E 02$ $11.52000-0.2291 E$ ก? 1. 72000 0.8197E 11.72000 0.4197E 02 11. $5200000-0.6276 \mathrm{E}-02$ $12.02000-0.4905 E 02$ $12.02000-0.4905 E$ O2 $-12.12000-0.3185 E \quad 02$. $12.22000-0.3417 E 02$ $12.32000 \quad 0.9646 E .01$ $12.42000 \quad 0.2312 E \quad 02$ 12.52000 0.1937E $12.62000 \quad 0.3465 F$ O 12 . $72000 \quad 0.2953 \mathrm{E}$ O 1 $12.92000 \quad 0.1835 \mathrm{~F} 02$ 13.02000 0.154]E 02 13. 12000 $0.3138 \overline{0}$ O2 $13.22000-0.1366 E \quad 02$ $13032000 \quad 0.2913 E$ OI $\begin{array}{rrr}13.42000 & 0.5472 E & 01 \\ 13.52000 & -0.9882 E & 01\end{array}$ $13.52000-0.9882 \mathrm{E} 01$ $13.62000-0.1311 \mathrm{~F} 02$ 13. $72000-0.1496 \mathrm{E}$ o I $13.82000 \quad 0.3535 \mathrm{E} \quad 2$ $13.92000-0.2929 E \quad 02$ $14.02000000027 E$ OI $14.12000-0.2720 \mathrm{E} \quad 22$ $14.22000 \quad 0.3311 E 02$ $14.32000-00850$ O $14.42000 \quad 0.2142 \mathrm{E} 02$ $14.52000-0.7870 \mathrm{OE} 00$ $14.72000 \quad 0.1575 \mathrm{E} \mathrm{O1}$ 14.72000 0.1575E ol $14.82000 \quad 0.3583 E$ OI 14.92000
$15.02000-0.591 \mathrm{NE}$ OD $15.12000 \quad 0.1303 E^{02}$ $15.220000-0.433 i E$ Ö $15.32000-0.1854 \mathrm{E} \quad 02$ $15.42000-0.3150 \mathrm{E} \quad 01$ 15.52000 0.203 IE 15.62000 0.26896 02 15.62000 0.2689E $15.72000-0.1524 E 02$ $15.92000 \quad 0.5591 E$ 01
$11.34000-0.1606 \mathrm{E} 02$ $11.44000-0.3575 E \quad 02$ $11.54000-0.1862 E-02$ 11.74000 0.4449E 02 11.74000 0.4449E 02 ii. $94000-0.2622 \mathrm{E} 02$ $11.94000-0.2622 E$ O2 $12004000-0.4612 E 02$ 12.14000 =0.3346E 02 $12.24000-0.3484 E \quad 02$ $12.34000 \quad 0.2283 E 02$ $12.44000-0.2067 E .02$ 12.54000 0.1350E 02 $12.64000 \quad 0.3031 \mathrm{E} 01$ 12.74000 0.17325 01 12.84000 0.7165E OI $12.94000 \quad 0.1886 E \quad 02$ $13.04000 \quad 0.1994 E 02$ $13.140000 .3736 \mathrm{E} \mathrm{O2}$ $13.24000-0.1677 \mathrm{E} 02$ 13.34000 0.1685E 02 13.44000 O.1752E 02 $13.54000-0.5315 \mathrm{E}$ O1 13.64000 -0.1059E 02 13.1\%000 0.4134E OI $13.84000 \quad 0.7047 E$ OI $13.94000-0.2122 E .02$ $14.040000 .2000 E .02$ 14.34000 O $5024 \mathrm{E} 02$ 14. $34000-0.3465 \mathrm{E}$ 0 $14.44000 \quad 0.1571 E$ O2 $14.54000 \quad 0.2360 E_{00}$ $14.64000-0.2862 E 02$ 14.74000 0.3858E OI 14.84000 - $0.2157 E$ O 02 14.94000 0.1614E O2 $15.04000 \quad 0.9724 E$ OL $15.14000-0.5510 E 00$ $15.240000 .1969 \mathrm{E} 01$ $15.34000-0.1429 E \quad 02$ $15.44000 \quad 0.1970 \mathrm{E}$ 15.54000 O. ITO IE 02 15.64000 0.6772E 01 15.74000 . $0.6740 \mathrm{~T}$ I $15.94000 \quad 0.2756$ E 01
$11.36000-0.1216 E 02$ $11.46000-0.4358 \mathrm{E}$ $11.56000-0.1311$ $11.660000 .2413 \mathrm{E}$ 11.760000 .4673502 $1.86000 \quad 0.0020 \mathrm{E} 02$ $96000-0.2185 \mathrm{E}$ $2.06000-0.4134 \mathrm{E} 02$ $12.16000-0.3306 \mathrm{~F}$ $12.16000=0.3306 E$
$12.26000=0.2114 \mathrm{E}$ $12.26000-0.2114 E 02$ $12.36000 \quad 0.1236 E \quad 02$ $12.4600 \mathrm{C} \quad 0.1398 \mathrm{E} \quad 02$ $12.56000 \quad 0.1134 \mathrm{E} \mathrm{O2}$ $12.66000-0.5827 E$ OI $12.76000-0.5709 E$ $12.86000 \quad 0.1677 \mathrm{E}$ $12.96000 \quad 0.759$ AE 13.06000 0.227? $13.16000 \quad 0.1358 \mathrm{E} 02$ 13.16000 0.1358E $13.26000-0.1638 E$ O2 $13.46000=0.1063 \mathrm{~F}$ O 1 $13.56000 \quad 0.3110 E$ OI 13.56000 0.3115E 01 $13.66200-0.1105502$

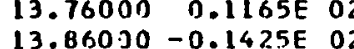
$13.96000-0.1299 E 0$ $14.06000-0.8660 E$ OO $14.16000-0.1461 \mathrm{E} \quad 02$ $14.26000 \quad 0.5465 \mathrm{E}$ $14.36000=0.8937 \mathrm{E}$ 14.46000 O.1772E D $4056000-0.4606 \mathrm{E}$ OI $14.66000-0.2279502$ $14.76000 \quad 0.5394 \mathrm{E}$ OI 14.76000 0.5394E O1 $14.86000=0.2185 \mathrm{E}$.

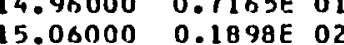
5.06000 0.1898E O2 $15.16000 .0 .7677 E$ O 15.260000 .94 A8E OI $15.36000-0.7677$ $15.46000 \quad 0.9055 E$ O $15.560000 .1354 E$ $15.76000-0.1299 \mathrm{E}$ O $15.86000-0.1319 E 0$ $15.96000-0.2480 E$ OI
$11.38000-0.1047 E 02$ $11.48000-0.3468$ 82 02 $11.58000-0.7835 E-01$ 11.78000 $0.4902 E$ O2 11.78000 0.490 02 $11.08000-0.46665 .02$ $1.5000-0.2728 E 02$ $12.08000-0.3622 E 02$ $12.18000=0.339 \mathrm{AE}$. 02 $12.2 \mathrm{AOOO} 0.2047 \mathrm{E} 01$ $12.380000 .9291 E$ OL 12.48000 .. 0.77560 01 $12.58000 \quad 0.1701 E$ O2 $12.68000-0.3031 E$ OI $12.78000-0.1244 E \quad 02$ $12.88000 \quad 0.1728 \mathrm{EE}$ $12.98000 \quad 0.8740 E$ OI $13.08000-0.2315 \mathrm{E} 02$ $13.18000-0.1772 \mathrm{E}$ OI $13.28000-0.1083 E 02$ $13.38000=0.1524 E \quad 02$ $13.40000-0.2744 E 02$ $13.58000-0.4528$ E 01 $13.60000 .0 .7974 \mathrm{E} 01$ 13.7A000 0.1354E 02 $13.88000-0.3913 E \quad 02$ $13.98000-0.5039 \mathrm{E} \mathrm{Ol}$ $14.08000-0.1925 E \quad 02$ $14.18000 \quad 0.3465 E$ O1 $14.28000 \quad 0.4697 \mathrm{fE}$ O2 $4.38000-0.322$ EE $4.48000-0.3228 E$ OI $14.68000=0.1047 \mathrm{E}$ 02 $14.78000 \quad 0.8701 \mathrm{E}$ 01 14.78000 0.8701E 0I $14.98000-0.1063 \mathrm{E}$ OI $15.08000 \quad 0.3083 E \quad 02$ $15.18000=0.9724 E 01$ $15.38000-0.7090 E$ OO $15.48000 \quad 0.1472 \mathrm{E} \quad 02$ 15.580000 .198802 $15.68000-0.2075 E$ O2 $15.78000 .0 .4685 E .01$ $15.88000-0.8583 E 01$ $15.98000-0.4724 \mathrm{E} 01$ 


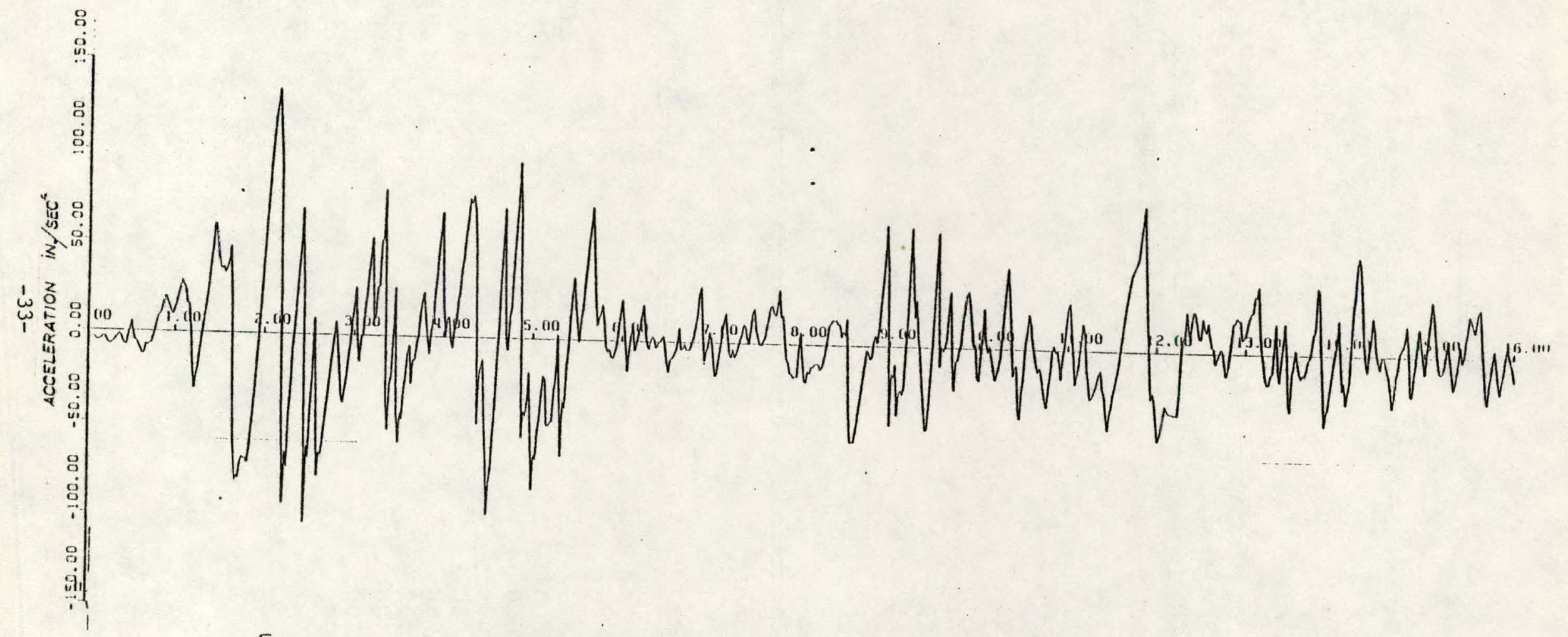

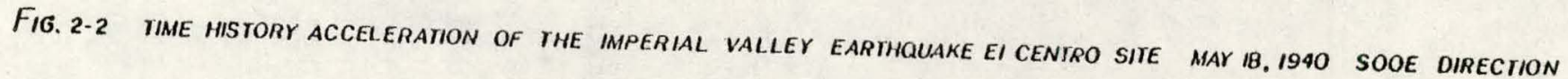


TABLE 2-4

Imperial Valley Earthquake, May 18, 1940

I. Acceleration Spectrum Before Modified (shown dotted Iine in Fig. 2-3)

Period

.0017

.0329

.0641

.0953

.1265

.1977

.1889

.2201

.2513

.2825
Frequency

588.24

30.39

15.60

10.50

7.91

6.34

5.29

4.54

3.98

3.54
Spectral Accel.

136.6073

333.9189

744.0145

342.2616

1186.5160

509.5554

665.5077

870.3499

631.5481

406.5282

II. Acceleration Spectrum After Modified (shown solid line in Fig. 2-3)

\section{Feriod}

.001698

.029600

.058000

.071000

.091000

.114000

.141000

.172000

.200000

.250000

.32300
Frequency

588.93

34.97

17.24

14.08

10.99

8.77

7.09

5.81

5.00

4.00

3.09
145.0

380.0

755.0

775.0

440.0

1188.0

1188.0

700.0

871.0

871.0

400.0 


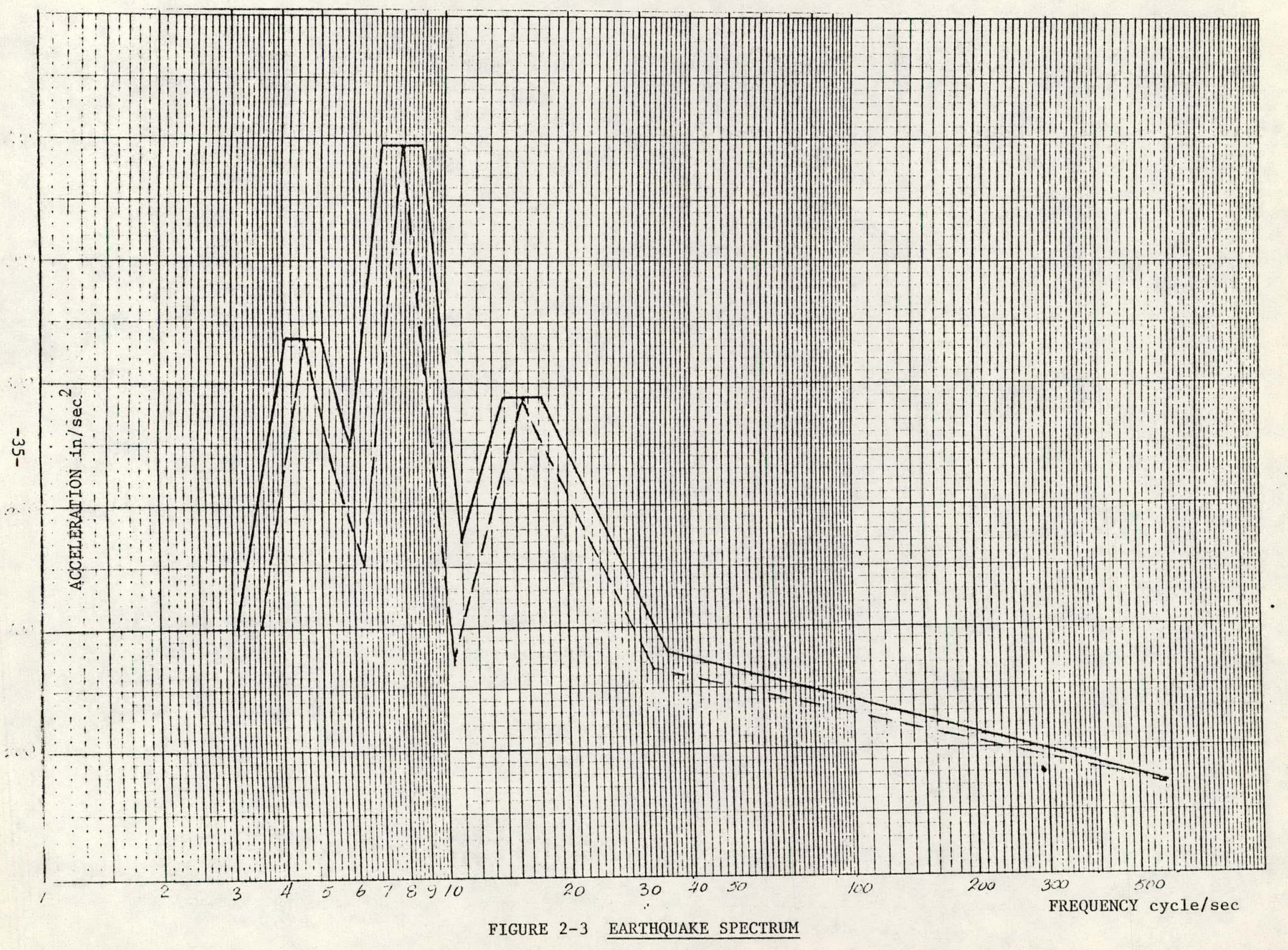


TAPLF 2-5 HOYGAAPI PROPLEY DYNAHIC CASE A TIIME HISTJRY ANALYSISI

NA I URAL FRE RUENGIE

\begin{tabular}{|c|c|c|c|}
\hline NUHASE & $\begin{array}{l}\text { FRI BUFNC. } \\
\text { IRAIISECI. }\end{array}$ & $\begin{array}{r}\text { FRINUFHCY } \\
\text { (CYCLIS/SFG) }\end{array}$ & $\begin{array}{l}\text { PEPIDO } \\
\text { (SCE) }\end{array}$ \\
\hline 1 & $.1743 \mathrm{~F}+113$ & .? $85,3 E+02$ & $.3504 E-01$ \\
\hline$? \cdots$ & $.35 f_{14}+5+03$ & $\cdots-5577=+02$ & -.17 \\
\hline 3 & $.5121 E+113$ & $-81511 F+02$ & $.1227 E-0 i$ \\
\hline ४ & . $3906 F+0.3$ & $.1417 E+03$ & $.71) 55 E-02$ \\
\hline 5. & AHE3E+0I & $\because 162 A E+13$ & $.5142 E-02$ \\
\hline
\end{tabular}


TAB.E 2-GA hOVGAARO PIPIING SYSTEM CASE A (TIMF MISTOPY)

MODE S IT APE

NOIJF R.1!NHEF 1

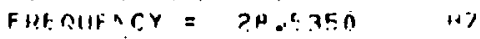

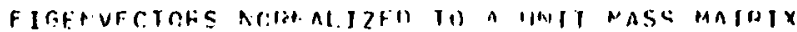

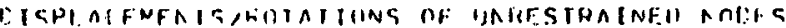

\begin{tabular}{|c|c|c|c|c|c|c|}
\hline $\begin{array}{c}\text { A:niHF } \\
\text { NUPAFAH }\end{array}$ & TlAM SLOTIOA- & 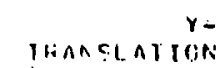 & Translotrinn & PUIAIICA & GrTAIJAN & - \\
\hline$=$ & 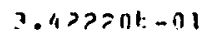 & $-\dot{c}$. hodrast. - na & 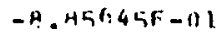 & $-? .704 .85 E-02$ & $-1.0 \cap \approx 4<E-\cap ?$ & $-1.0731 .36-0 \bar{\varepsilon}$ \\
\hline$?$ & $1 . n I A C D F+n 0$ & $-1,13+6+4-1)^{4}$ & $-2.5(.8798+00$ & $-3.37631 E-02$ & $-2.011490 \mathrm{n}-02$ & $2 ? E-0 \overline{6}$ \\
\hline 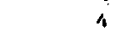 & 1. & -1 . Inckstit- -01 & $1065>r+00$ & $-7.55499 E-02$ & $7>273 \mathrm{~F}-02$ & IOTE - O? \\
\hline a & ח & $-7.7 n B_{1}<\geqslant 1-01$ & $-2 . A>144 F+00$ & -2. กค34.hE - O2 & $-3.671 \times 9 E-0 ?$ & $17444 E-02$ \\
\hline f. & $1.3 P>$ IAF & $-2.7 .2+03 t-01$ & $-1.447975+110$ & -1.116 & $-3.837 n 4 F-112$ & $1564 \mathrm{HE}-\mathrm{n} ?$ \\
\hline 7 & $1.2+164 E+0 n$ & $n \mid<t .-n\}$ & $-1.25435 F+0 n$ & $-1 \cdot n$ & $1 \leq 31 f-02$ & -03 \\
\hline $\boldsymbol{\mu}$ & 9. SRQOกF -01 & $-2.4 \times 1=40-01$ & $-3.11 .143 .35-n 1$ & $-1.74720 \mathrm{OF}-02$ & $-3.2 P \in \geq ?\{-\cap>$ & $-1.57579 F-103$ \\
\hline 9 & $3.57 \cap 79,0-01$ & $-1,2 \pi \rho, 2 \pi t-01$ & -1.3191 AF -0.3 & $-5.79075 F-03$ & $-1.61=>7 E-0>$ & 3E5 5 SAE -03 \\
\hline $\ln$ & $9 .\{c 5 \mid\}-n>$ & - & $: 4614 \mathrm{~F}-04$ & $-3.07759 \mathrm{~F}-03$ & $114 E-03$ & $\approx 77 \mu F-04$ \\
\hline
\end{tabular}

M(II)F NINHEF 1

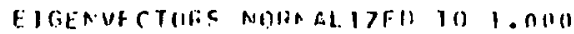

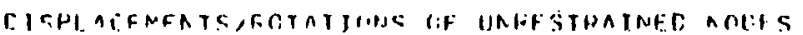

\begin{tabular}{|c|c|c|c|c|c|c|}
\hline $\begin{array}{l}\text { NuINF: } \\
\text { MINARED }\end{array}$ & 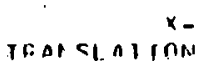 & 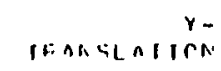 & $\begin{array}{l}7-. \\
\text { IUANSLATION }\end{array}$ & Porration & CTATION & 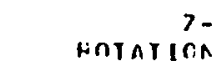 \\
\hline 1 & n. & r. & n. & n. & $\cdots$ & \\
\hline 2 & 1.07 (1605-D) & $-1.71049 t-0.5$ & $70 \in 5 F-01$ & $-A .14 G 23 E-03$ & $3+n+f-0.3$ & $-\cap 3$ \\
\hline 3 & $3.1415 \% F-01$ & $-z . c a: \subseteq \neg f,-0=$ & $-8.34905 F-111$ & $-1.1156255-112$ & $-6 . \bar{\varepsilon} \div \div 12 \mathrm{~F}:-0.3$ & 17598.03 \\
\hline 4 & $4.0+7.35(x-n)$ & $-3.4403 n t-0 z$ & $-1.0 n g n \cap F+00$ & $-H_{.}$T0549F -03 & $=78-n .3$ & $-7.94405 E-03$ \\
\hline 5 & $4 . ?=440 \mathrm{~F}-01$ & - - .ner.pif $-n>$ & $-F .2 n n 97 f-n]$ & $-6 . S 1790 E-03$ & eratt $-n$ ? & $-1, E 1 A$ \\
\hline$\theta$ & $4 . ?=4 \cap|F-n|$ & 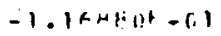 & $-6.09402 F-01$ & -5.41 & $-0 ?$ & $-1 . .3 n$ \\
\hline 7 & $4.3 .37 \mathrm{Ft}-\mathrm{nI}$ & $-1.2134 \mid+01$ & $-3.9741 \mathrm{OF}-01$ & -5.107 & -1.215 & $-1 . n n$ \\
\hline H & $3.1=40 C F-01$ & $-1 . n P D \cap I F-n 1$ & $-9.43003 F-n ?$ & $-3.4 n 173 f-03$ & 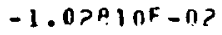 & $-4.9 ? 970 F-114$ \\
\hline ? & $1.117058-01$. & $-3.40 \leq 5 a t-1)$ & $-4.1>6025-114$ & - -"11 & $-5.042 P>E-0.3$ & $-4.3235 A A-04$ \\
\hline 10 & $7.00174 E-0 ?$ & $-1 . n=44-3 t-41>$ & $-2.010 .354 f-0.4$ & $-0.6779 .3 E-0.4$ & $-2.7+P O Q E-\cap 3$ & $-2.1 \times 7 P 9 F-n 4$ \\
\hline 11 & n. & $n$. & c. & $n$. & & $n$. \\
\hline
\end{tabular}


TARLE ?-G HOVGABRD PIPING SYSTEM CASE A (TIME HISTOAY)

MODE S I A P E

NOLIF. MLUHE E. ?

FHF DIFACY $=$ GE. $173 \% \quad \mathrm{~m}$

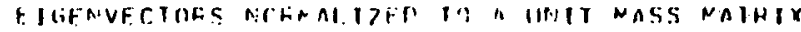

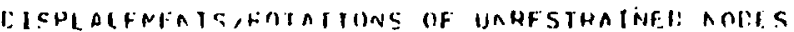

\begin{tabular}{|c|c|c|c|c|c|c|}
\hline $\begin{array}{l}\text { NOUNF } \\
\text { AINATFER }\end{array}$ & TEAR CLOYTUAT & 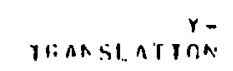 & miansiarmón & a & HCTAIION & מ- \\
\hline$?$ & $9.04 P 55 E-01$ & $-z .0114$ it. -24 & 7. ?.729nF-n1 & ?.133765F-02 & $1.39 / 1>F-n 2$ & $-2.545 H G F-02$ \\
\hline 3 & $2 . \approx 123 \mathrm{FF} \circ \cap n$ & -4.1 cnari.-14 & $1.7 f: 570 F+01)$ & $1 . “ P O P A F-0 ?$ & 2. IIISTCF -0 ? & $=-02$ \\
\hline 4 & 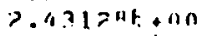 & $-5.91+14 t:-11\rangle$ & $1.569278+0 n$ & i. ..$H ? 64 F .04$ & $t 7 \equiv \cap \cap F-11 ?$ & $F-0 . ?$ \\
\hline 5 & $2.4>n A=1:+\infty n$ & $-3.34+3(4 t)-11)$ & $5.76747 F-01$ & $-1.4 A 105 f-03$ & $>S A \cap E-\cap ?$ & IODF- -03 \\
\hline 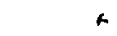 & $2.41 B>A F+O n$ & 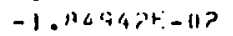 & $-1.94269 F-07$ & $-1.17404 \mathrm{~F}-03$ & ?. .75 .1 DE - O? & $I 7 E-n \dot{4}$ \\
\hline 7 & t:, no & $43 i-0 ?$ & $-n !$ & $-\mu . G_{7}$ & $E-n j$ & $019 \mathrm{E}-04$ \\
\hline 9 & z. Bansefon & 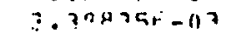 & $-4.7 F>i$ if $-0 i$ & $-3.09 \geqslant 0 \mathrm{OE}-04$ & $76535 F-07$ & $4 . \cap \cap 31$ OF -05 \\
\hline 9 & $1.1074 .7 F+00$ & $4.394 .394-07$ & Z. $9675 n F-0.3$ & $1.1 .36476-04$ & -4.4 C.A5E - त? & $-4.559+0 F-05$ \\
\hline 10 & $3.735117-111$ & $1.617 \div 28:-117$ & $1.4 \times 3975-113$ & $1.19205 E-04$ & $-3.07772 .8-n 2$ & 79 HOF -05 \\
\hline
\end{tabular}

NOIIF NII NAHR:

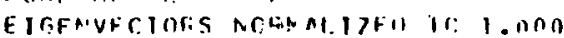

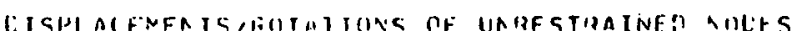

\begin{tabular}{|c|c|c|c|c|c|c|}
\hline 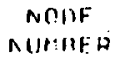 & 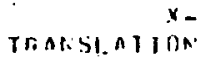 & 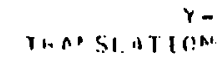 & JHANSLATIUIN & portation & $\begin{array}{l}r- \\
\text { I IOR }\end{array}$ & " \\
\hline 1 & 11. & $n$ & n. & $n$. & 0 . & $n$. \\
\hline ? & $3.1217 \% \mathrm{t}-\mathrm{M1}$ & $-F .514$ at -05 & $2.97493 F-111$ & n. $364<$ nft -03 & $5.7 .34118-03$ & $-1.04713 E-0 ?$ \\
\hline 3 & 1. nsigant $-n 1$ & -1.7.71:E-94 & $7.26 \geq 41 F-01$ & 5.44 .39 AE - 03 & $1.1 \angle+P=F-0 ?$ & $-7.36 .25 n E-n 2$ \\
\hline 4 & $1 . n n n n c e+0 n$ & $-\overline{6} .433 ? 40,-02$ & $6.4544 \mathrm{MF}-01$ & К.4Pח7IE-กS & $1.51032+0$ ? & ?.7635E-n4 \\
\hline 5 & $0.50, f<C l-n)$ & $-1.275 ; 8 \leq-112$ & 2.372 Iff $-n 1$ & $-6.03 \mid t \in f-04$ & $1.4517 \mathrm{PF}-07$ & $1 \leq H 2 A E-04$ \\
\hline r & B. SAF 4 GF $-(1)$ & $-7 . G r+7$ HE -07 & $-7.579 n A F-03$ & $-4.4 . \supset A 9 I F-04$ & $1 . \ddot{c}>\leq \geq \geqslant F-n ?$ & P.APA?KF -04 \\
\hline 7 & $0.933744 \mathrm{t}-\mathrm{nI}$ & $-=314+1<+-113$ & $-1.9 R \cap 7 y=01$ & $-? .5 \mathrm{G}+16 \mathrm{~F}-04$ & B. $2114.3 E-0.3$ & $=.0 \div 7 \times 1 E-\cap 4$ \\
\hline $\mathbf{p}$ & $4.2+179,5-111$ & 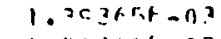 & $-1.67076 F-01$ & $-1.27166 f-04$ & -1.3350 FF - D? & $1.64650 E-n 5$ \\
\hline ; & $4.5=\sin 5 \dot{E}-n 1$ & 1. $001 i_{0} ; 4-0$ ? & $1.37055 F-117$ & 4.0 .751 UP. -05 & $-1.81 \equiv \mid[F-n\rangle$ & $-1.275 .39 F-n 5$ \\
\hline in & 1.5JH> IF $-n 1$ & $t .+501725-04$ & c. $10765,5-04$ & 4. HAIHAF-1)E & $-1.2+5, p \mu F-0>$ & $-9.37694 E-n E$ \\
\hline 11 & $n$. & $r$. & 0.. & 11. & $n$. & $n$. \\
\hline
\end{tabular}




\section{TAGLE 2-6C HOVGAAAD PIPINO SYSTEM CASE A (TIME HISTORY)}

MO DE S H A P E - ...............

MOIIE NINHEF ?

FHF UIIEACY $=41.6$ OIO

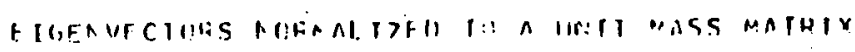

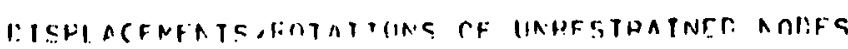

\begin{tabular}{|c|c|c|c|c|c|c|}
\hline None. & $x-$ & $\gamma-$ & - & $x-$ & HCIATION & Hothat \\
\hline AtJNIAER & THAl. St Q O TOR: & THIASI : I IUUPi & THANSSLATION & $\begin{array}{r}\text { HOTATTOA } \\
01245 F-0 Z\end{array}$ & $\begin{array}{l}\text { HCIATION } \\
-4 . \text { SR:TECF }-04\end{array}$ & $1.97079 E-\cap \bar{E}$ \\
\hline$?$ & -9.1175 ?E -01 & $-1.9 ? 450$ - 02 & 4. คคว5|F-111 & $\begin{array}{r}1.01245 F-02 \\
-1.1717 A F-03\end{array}$ & $-9.93 \leq>95-04$ & $1.5554 \mathrm{AE}-0 ?$ \\
\hline 3 & 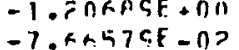 & 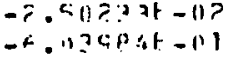 & $\begin{array}{l}5.7043 A F-n 1 \\
2.54054 F-n ?\end{array}$ & $\begin{array}{l}-1.0717 G 5-02 \\
-3.6007 G F=02\end{array}$ & $2.31 \geq 275-03$ & ดว $9 \mathrm{~F}-\mathrm{n}$ \\
\hline 5 & $0.5 n 45 \times 1-01$ & 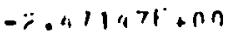 & $-4.491 \cap n F-01$ & $-5 .>19+5 E-02$ & G. $P$ P PQE -05 & TARIL $-\cap ?$ \\
\hline 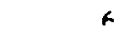 & 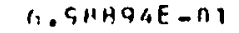 & $-? . G A 1(14 F+\cap n$ & $-4.1+1915-01$ & $-6.1) A A \cap B F .02$ & $-7.77 F+? F-n 3$ & $-5.4119 \mathrm{AF}-\cap \mathrm{Z}$ \\
\hline 7 & A. SPOCAF -01 & $-4.75<51 t+0.2$ & $-3.90744 F-111$ & $-3.45650 F-02$ & $-5.60373 F-03$ & $-7.90026 F-0 \bar{E}$ \\
\hline N & S.PO96:3F-OI & $-? .9 \pi ? n$ at +0 (1) & $-1.3961 / 4 F-111$ & $-8.6 \times 5 ? 7 F-02$ & $-1.27 \div 04 \mathrm{E}-0 ?$ & $1.211255-04$ \\
\hline c) & $\because 0451=t-01$ & $7<\{95 t+13 n$ & $-8.91145 \mathrm{E}-619$ & $-4.95974 F-02$ & $-1.030 ? \cap F-n$ ? & $053 F-n 3$ \\
\hline 10 & $1.6714 P E-112$ & $-5.776764-01$ & $-4.407 .365 .-114$ & $162 F-02$ & 0.1 & -112 \\
\hline
\end{tabular}

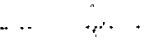

NODF in:1 Mititia 3

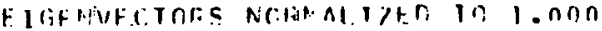

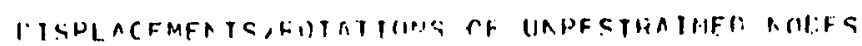

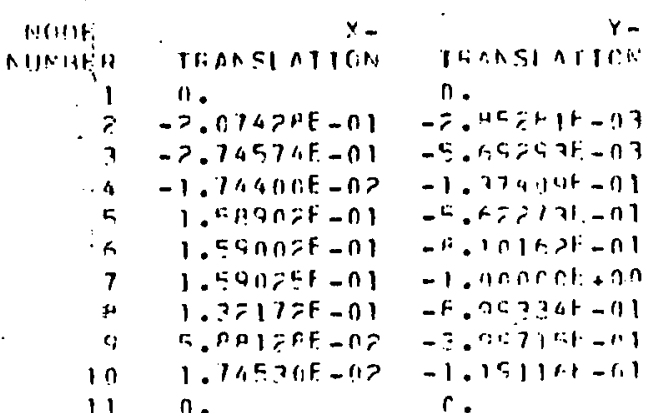

TIAASLATIIIN

1. OR $5>9 F-01$

$1.4) 15(1) F-111$

$5 . \sin 033 F-0.3$

$-1.11272 \%-01$

$-1.0 n=0,1 F-01$

$-8.116 G$ HHF -0 ?

$-3.176>211 \mathrm{~F}-0$ ?

$-i . n n 476 F-114$

$n$.
Wothtion

?. $30337 \mathrm{E}-03$ $-1.45912 \mathrm{~F}-03$

$-1 ! .2 F \geq 9>F-03$

-1.1 AILOF-OR

- 1. 3aSin ZF-OZ

- 1. जн264F-0?

$-1.071345-0 ?$

- 1 . TP. 3 Bf - n2

-1 . $1 \sin 4 F-0 \bar{c}$

$n$.
hCt TATIOAM 0 .

-1.1301GE-O4 $-2 . \overline{2} \cdot 0.73 \mathrm{~F}-\mathrm{n} 4$ S.CA=POF $-O 4$ 1. $56 .=5>F-05$ $-6.32151 F-04$

$-1.295 ? 5 F-03$ - 2. HOZ9FF-113 - 2. 34 ? 9 QF -0.3 $-1.40194 F-0.7$ 0 .
.4P365E- ก - $.6 Z 9 A \cap F-0 ?$ $-1.5 ? \backslash 17 F-n$ ? $-1.45671 E-n$ ? $-1 . ? 3125 \mathrm{~F}-n$ ? - . 973 PAF -03 5. $7794.35-04$ 2. $98092 F-04$ $n$. 
TABLE 2-60 HOVGAARO PIPING SYSTEM CASE A (TIME HISTOAY)

NOE S I A P E

Nothe nilmeitis a

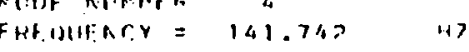

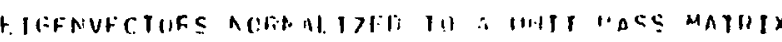

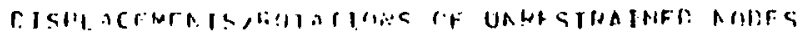

\begin{tabular}{|c|c|c|c|c|c|c|}
\hline $\begin{array}{l}\text { Mustr. } \\
\text { numented }\end{array}$ & TraAsin T100: & 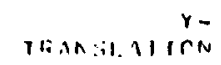 & ranaslatrós: & 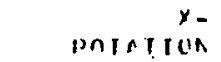 & Herarina & מotion \\
\hline ק & 1.t.Jn|SF-(1) & -t. 3rasunt:-0? & $-7.50570 F+100$ & $-J .47 K>7 F=02$ & $-2.7+00.75-n ?$ & $-2.10 \cap \cap 2 E-03$ \\
\hline$?$ & P.4PIRFE -111 & $-1.25=12 t-11>$ & $-1.71 \%$ \&AF $+10 n$ & $7.0>014 t-n 2$ & n? & -04 \\
\hline 4 & ?.1211??E-01 & $-6 .>141>1 . .07$ & $1.790155+110$ & $1.1071 .35-111$ & $6>35-3 ?$ & $693005-03$ \\
\hline 5 & $4.74 B 7+F-(1)$ & $-\Xi, 1 \in(., P(3+.,-1)$ & ?.7n?5KF. On & A. $.34444 F=02$ & 1. $C P+A F E-\cap ?$ & $-1.344 h 4 f-02$ \\
\hline 6 & $4 . B 1$ ACFE $-\mathrm{OI}$ & $-f . n=67 n 1-11)$ & ?.11?Pns + nn & h. 4 ABOIF -02 & $7774 A F-0 ?$ & ก \\
\hline 7 & $4 .|P>37 E-0|$ & $-7.52797 E-01$ & E.0? IOAF $+11 n$ & 4.467539 & $6: t 35420 t-0 ?$ & $-5.575 \times 35-0 ?$ \\
\hline 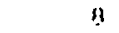 & I. nCac7t in & $-r_{.}|\subset| n>E-0 \mid$ & $\bar{E} .21 .237 \mathrm{~F}-\mathrm{nI}$ & $9.954 ? 15.03$ & $4.495 \subseteq \cap E-\cap ?$ & $1: \cap 4 B 46 F-\cap \bar{\varepsilon}$ \\
\hline $\mathbf{q}$ & 1.1514 & $-5.5+23$ & $\overline{2.2 A+7.3 F-118}$ & -1.208 & $-3.04 P>P F-n 2$ & $0 \in 7.375-02$ \\
\hline 10 & $4.85617 E-D)$ & $\Rightarrow$ - $\Rightarrow$ r. & $1.144465-10 ?$ & $-1.5761 A F=02$ & 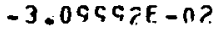 & $=.31184 F-n 3$ \\
\hline
\end{tabular}

molif Nindigk 4

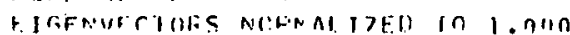

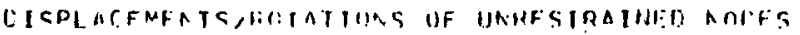

\begin{tabular}{|c|c|c|c|c|c|c|}
\hline $\begin{array}{l}\text { Ne!n)F } \\
\text { NUI:HF }\end{array}$ & of & r- & $\begin{array}{r}\ddot{7}- \\
\sin \operatorname{tin}\end{array}$ & $\begin{array}{l}x- \\
\text { Iun }\end{array}$ & $\begin{array}{l}Y- \\
O N\end{array}$ & ind \\
\hline 1 & n. & r. & 0. & $\pi$ & & 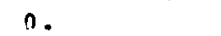 \\
\hline ? & ¿.4nวमFF-n? & $-1.7 n 314:-03$ & $-7 . \cap>|A \cap F-n|$ & $-1.04705 F-02$ & $\{=439 F-n 3$ & $-p$ \\
\hline 3 & H. $=4 \cap S T F-n>$ & $-2.34 \div 300^{\circ}-0.3$ & $-4.6>\& 4 a,-\cap 1$ & 1. HO946F. $=n 2$ & 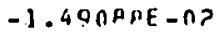 & $-1.4 \subseteq 730 E-04$ \\
\hline 4 & H. A5 $45 N E-O=$ & $-1.47+40 i-n 2$ & $4.117491 \mathrm{~F}-01$ & 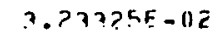 & $-1.65459 \mathrm{~F}-0 ?$ & $E-n 2$ \\
\hline 5 & $1.147 c=E-01$ & $-1.1117>4 t-111$ & $1.0 \cap n \cap \| F+n 0$ & $P .254 / 9 t-02$ & $27 F:-0.3$ & $-n ?$ \\
\hline A & 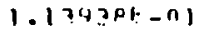 & $-1 . A=\mid 7 a f-111$ & $9.4 n$ & 1.73 & 1.200 .37 & - P. P. \\
\hline 7 & $1.1=0<c+-n i$ & 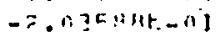 & $5.45 A R \mid E-(1)$ & $1.2 n+A+1 F-n 2$ & 1.e $4 \times 14 F-n ?$ & $-1 . A 1302, F-n ?$ \\
\hline$n$ & 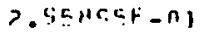 & ו & +.1 & 7.581147 & $1 . \bar{\varepsilon} 1$ & $F-n ?$ \\
\hline c & 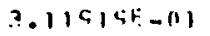 & - & $\therefore .176$ nat -07 & $-4.144645 t-03$ & $-A .2 .17$ & $.2+97$ \\
\hline 111 & $1.14 ; 5=5-111$ & -5.. $\div \leq 77 t-1>$ & $3 . n$ noquF - 13 & $-4 .>47 n>5-113$ & $-A .37 \div 37 \mathrm{~F}-0.3$ & $1.434 \mathrm{~K} 4 \mathrm{~F}-\mathrm{n} 3$ \\
\hline 11 & & $r$. & & $n$. & n. & \\
\hline
\end{tabular}


MONF NM HETFE

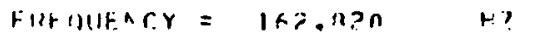

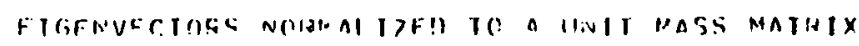

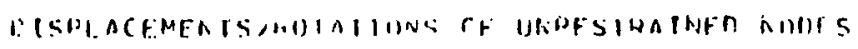

\begin{tabular}{|c|c|c|c|c|c|c|}
\hline 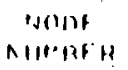 & TEAnCtaIJOAN & 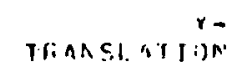 & TUNASLATUUNA & $\begin{array}{r}x=- \\
\text { HUITITIOA }\end{array}$ & PCIATION & poration \\
\hline$?$ & $-3.5 \times 947 E+0 n$ & $1.457=11-03$ & $-2.507>4 F-11$ & $->$. GOIก?F-03 & $5.530 .53 f-n 3$ & $1.04174 F-n 2$ \\
\hline כ & $-1.541<.2 E+n n$ & $? .4 .8144 F-0.7$ & $-1.7 n 99 \mid F-n ?$ & $1 . n+414 F-n 2$ & $1.1 n+1 \mid E-0 ?$ & $-\rho .775 .39 F-n \hat{\varepsilon}$ \\
\hline 4 & $1.145>1 E+10 n$ & $-1 . n+51$ At. $011 n$ & $n F-i l$ & -02 & n? & $8 F-n E$ \\
\hline 5 & $1.4973 .46+010$ & $-1.2 n$ ar $1:$ inn & $1.550745-01$ & $? .41 \geq 3.3 F-02$ & $1.4 n \angle>A F-n ?$ & $=.91941 \mathrm{~F}-02$ \\
\hline a & $1.5046+5+00$ & $1.2+1, f)-(:)$ & $-P . .45964 F-0 ?$ & ?. . $\because \cap 020 E-02$ & $1 . \varepsilon>t>3 F-0 ?$ & P. $34935 E-02$ \\
\hline 7 & $1.511911 E+00$ & $1.7 ? 411 A F+11 n$ & $-2.7+960 F-01$ & 3.147 & $E-n 3$ & IE-n? \\
\hline 4 & $1.44624 E+00$ & $2.19723 \mathrm{~F}+00$ & $-7 .>A \cap 86 F-01$ & $5.49143 .3 F-02$ & $Z E-0 ?$ & $5.48752 E-0 \hat{\varepsilon}$ \\
\hline 3 & 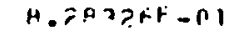 & $1.820731+0 \pi$ & $-6.0746: 45-11 d$ & G. $月>44 \Omega F-02$ & 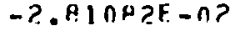 & $2.72014 \mathrm{~F}-02$ \\
\hline & $.73 \times 365-111$ & $t .>4[$ \&ARt $-n]$ & $04115 F-n 4$ & $.90 .7 n 6 F-02$ & $15497 E-07$ & $1.36043 \mathrm{~F}-\mathrm{n} ?$ \\
\hline
\end{tabular}

Nilile militith

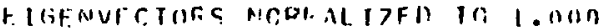

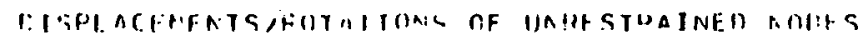

\begin{tabular}{|c|c|}
\hline $\begin{array}{l}\text { Nonit } \\
\text { M(NetFB }\end{array}$ & 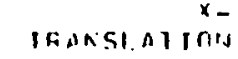 \\
\hline 1 & n. \\
\hline 7 & $-1 . n \cap \cap n \cap t+n \cap$ \\
\hline 3 & $-10.71400 F-111$ \\
\hline${ }^{4}$ & $? .50 ? 7 \subset \mathrm{F}-\mathrm{nI}$ \\
\hline a & $4.579755-111$ \\
\hline+ & 4. $\{n ?|<E-n|$ \\
\hline 1 & $4 .+|57+1:-0|$ \\
\hline ! & $4.4>34 f E-101$ \\
\hline a & $\partial . \leq \partial x=t-n$ \\
\hline $\ln$ & 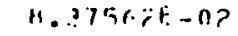 \\
\hline
\end{tabular}

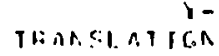
$n$.

$5.0 .00478-04$ $1-1=\operatorname{annt}-13$ -2.67 hisit:-71 $4 \cdot 1<\ln n t-n>$ c. ondirtat $-n$ c. $7 a_{14}: 1:-1 .-41$

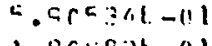
r.

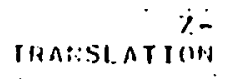

$-7.943935-0 ?$ -5. ? P A OOE - 0.3 Q. 3?H?7F-n 4.743 Int $-n$ ? $-? .7 \cap 9 A] F-0$ ? $-\mu .4 \%$ UOF - O $-6.97 t$ ?LF $-n$ $-1.55790 F-014$ $-5.30165 F-115$ n.

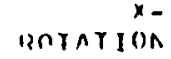
$n$.

$-P .20017 F-04$ 3. 315SGL-03 h. $477+4 F-03$ 7. $1,033.3 F-03$ I.กก.วगमF-ก $1.14623 \mathrm{3F}-02$ 1. HAI T? - OE ว. $118149 F-02$ 1. $5>71 \mathrm{At}-02$ $n$.
HCTATIOA 0 . . $69157 F-n ?$ 3. $3 P ?$ ? $4 E-n 3$ 4.035 4F -03 $4.295 \cap A F-03$ $3.7=051 \mathrm{f}-03$ 2.4 !: 45, > -03 $-4.34+34 F-03$ - $-5.5971 \times E-07$ $-6.541)$ GF. -03 n.
$7-$ $n$. ?.?0!544F-n2 1.P $1 \cap+3 F-n \hat{E}$ 2. $55373 F-n \bar{E}$ $=.7 \ln 41 F-0$ ? $1.67841 \mathrm{~F}-02$

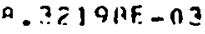
4. 1f.099F $-n$ ? $n$.

$-$

\section{2}

.




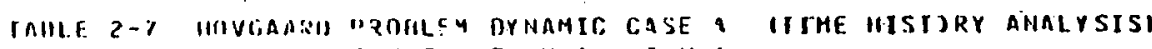

O I S L A : E IIE Y T HAXIH

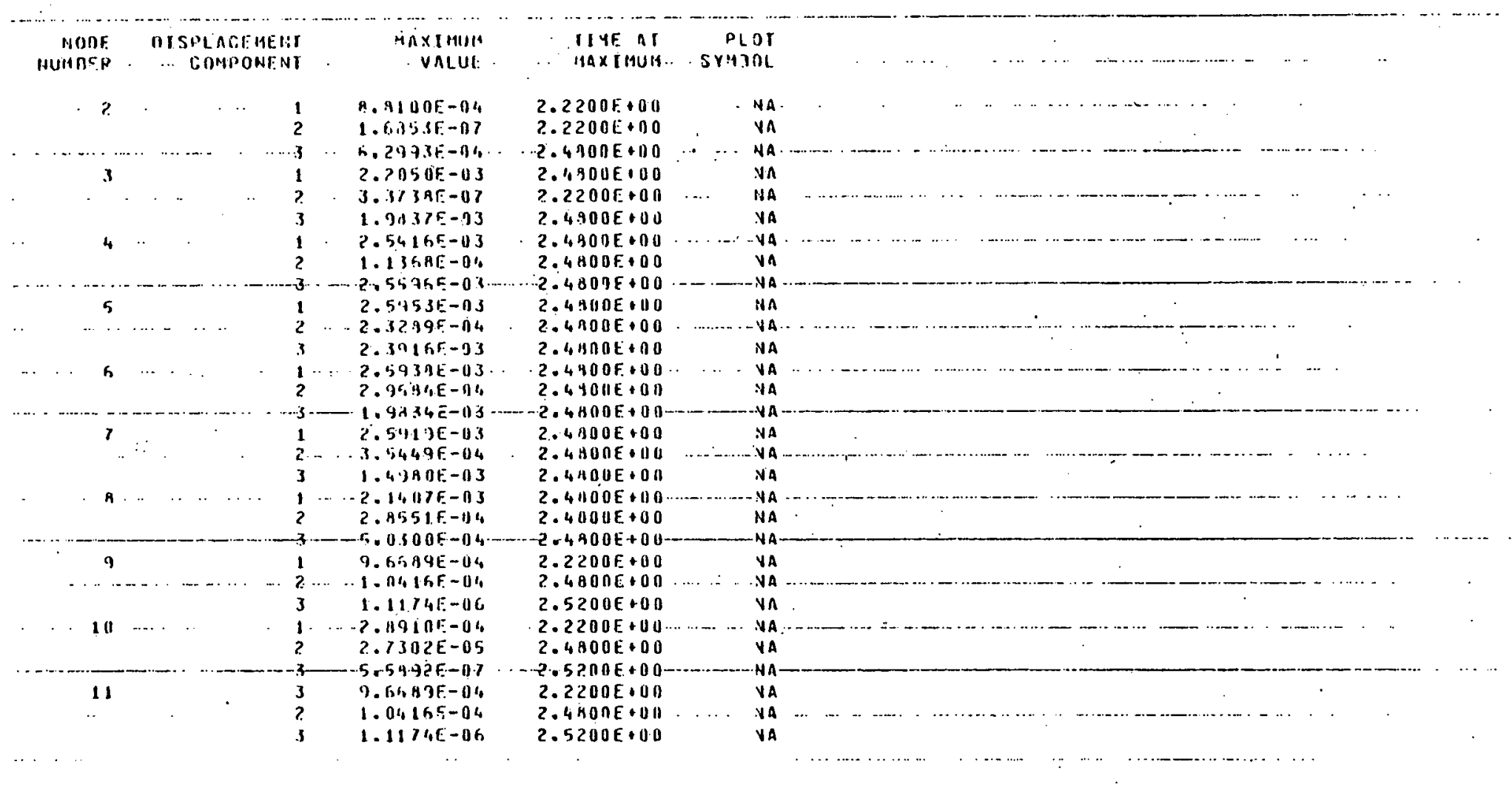


IAILL I-H IIIVGANRI PPOILEY OYNAMIC CASE I IIIHE HISIJRY AHALYSISI

$$
\text { F. O C F C OPUNEN T M.A.XIMA. }
$$

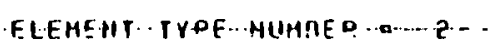

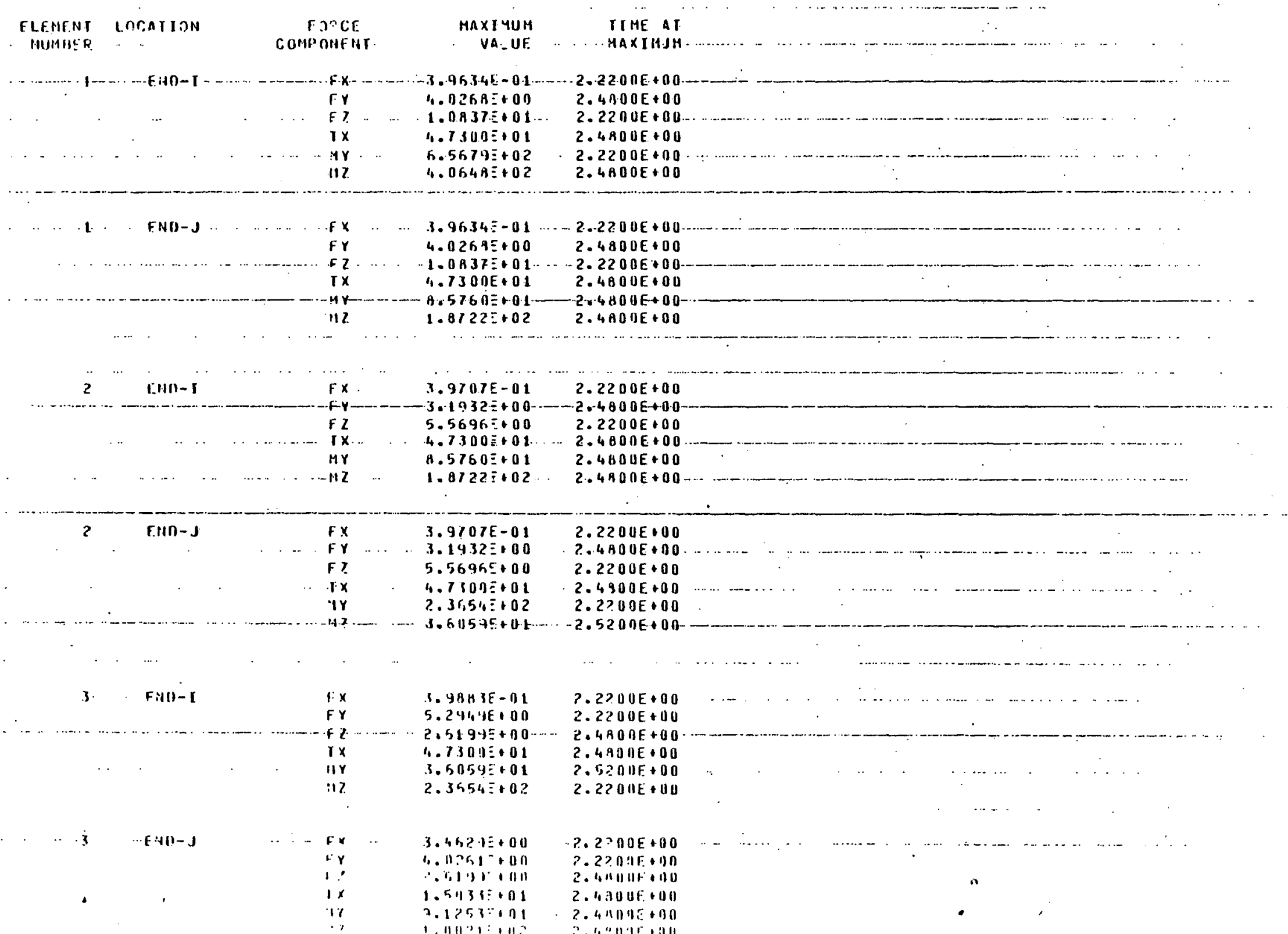




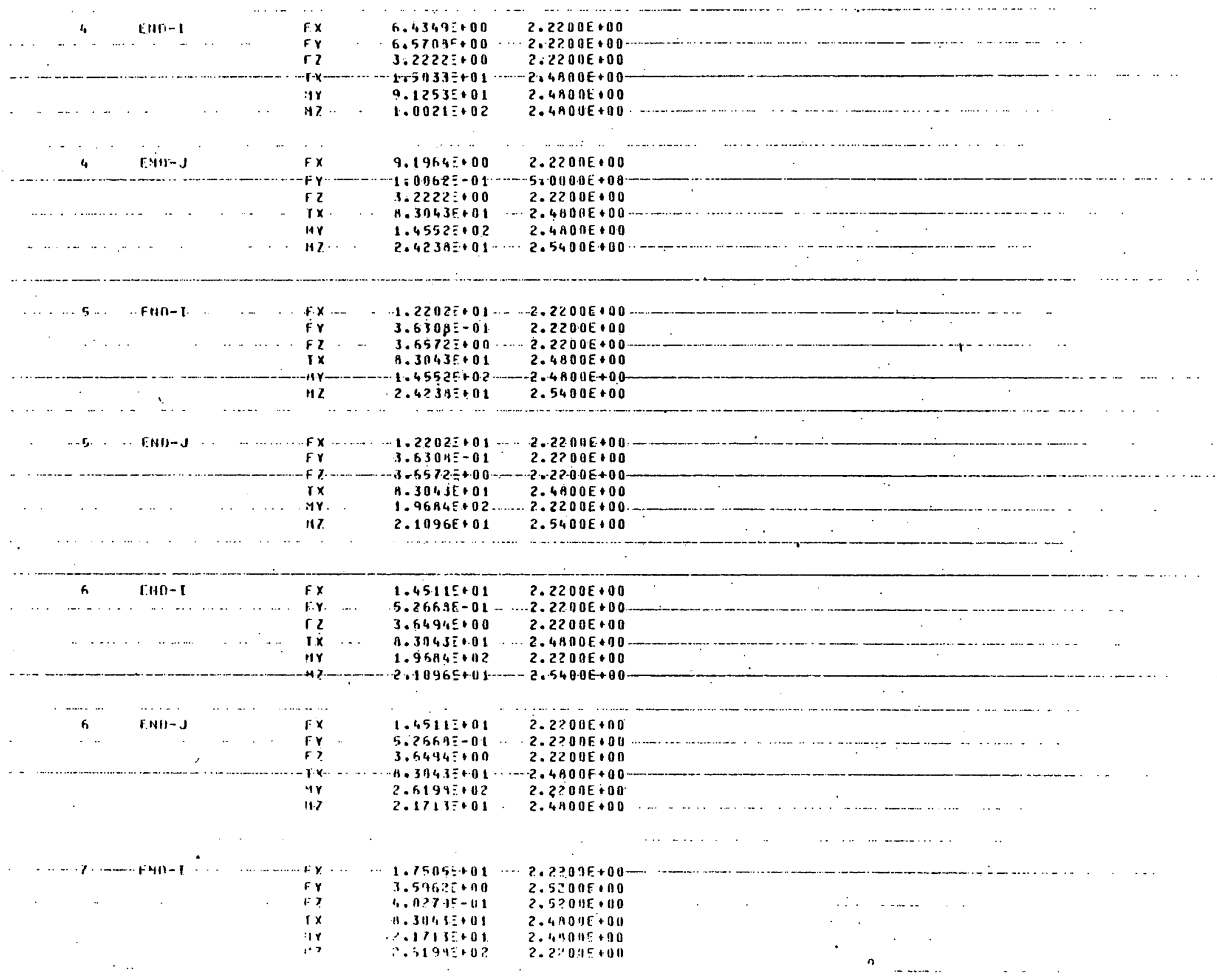




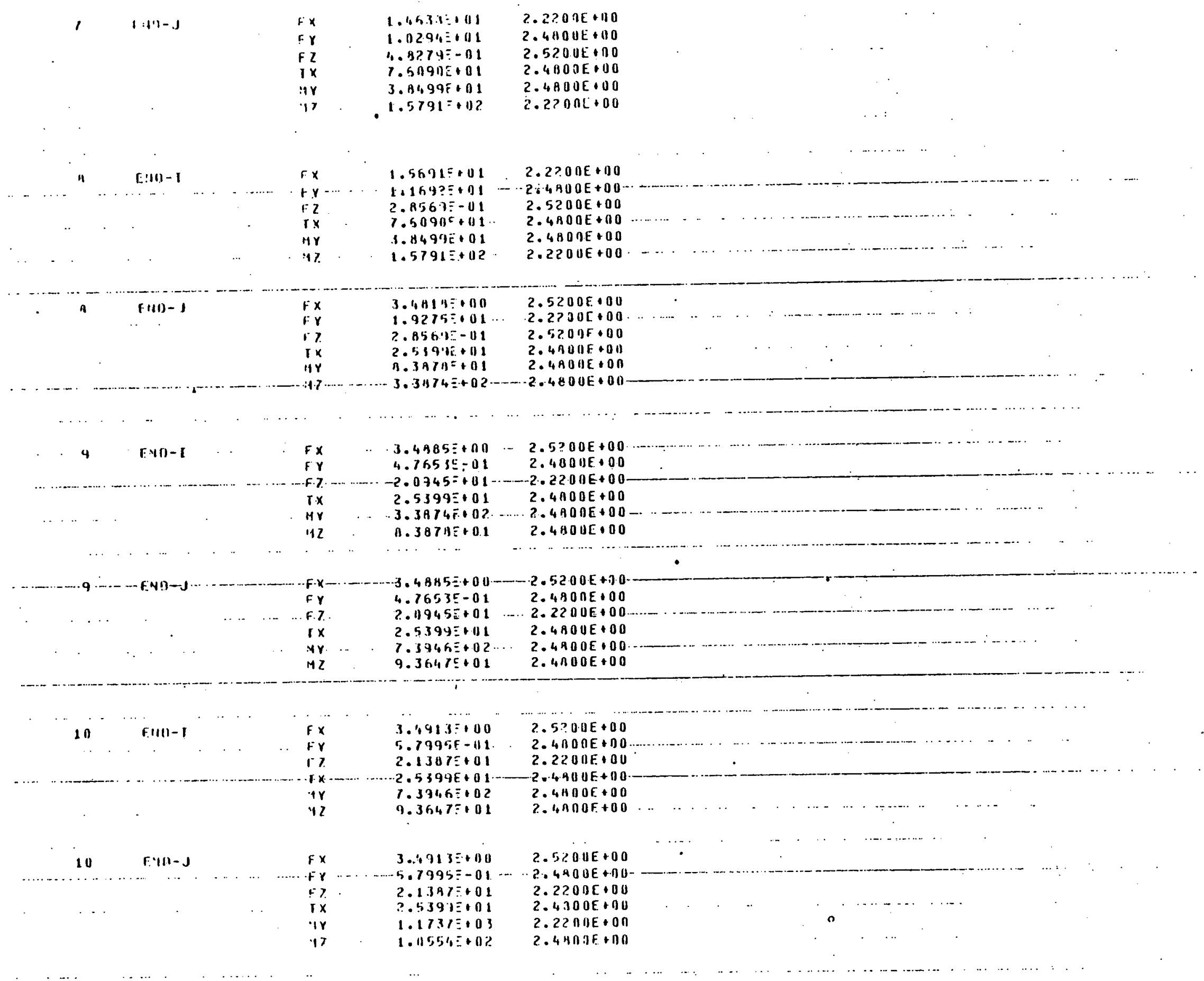


IAIIL 2-9 HJUGAARO PROBLEY MYHAHIC CASE A IIINE HISIJRY ANALYSISI

$$
S 1 \text { KES S R O H P O NENT HA X-I M.A. }
$$

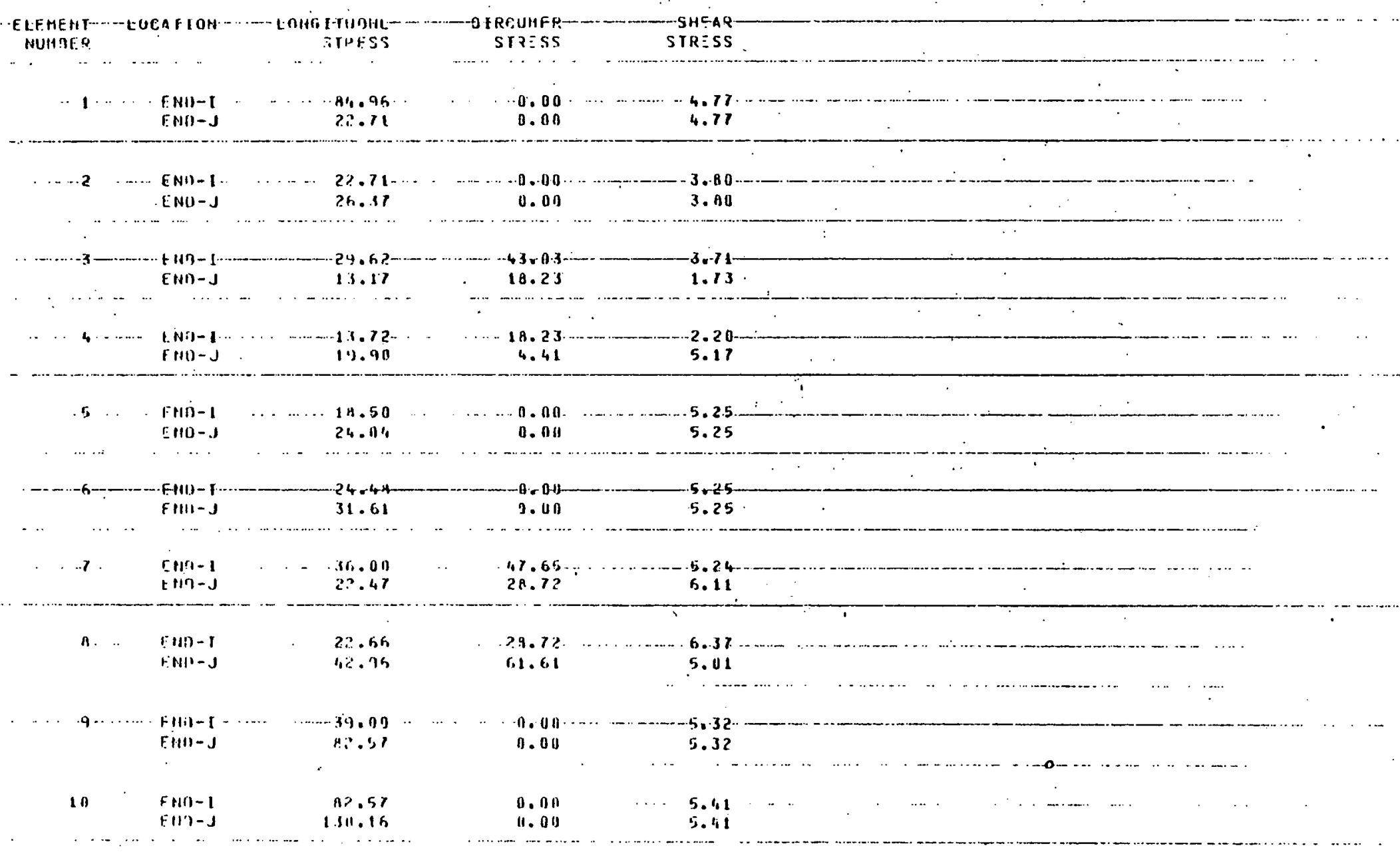


TAGLE 2-I0 HOVGAARO PIPIAG SYSTEN CASF B (RESPONSE SPECTAUM)

MCDAL PARTICIPATION FACTORS AND EARTHOUAKE RESPONSE -

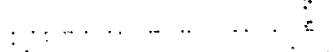

\section{DIPECTION FACTOFS}

$$
x=1.000 \mathrm{C}=-0.0000 \quad 7=-0.0000
$$

INDICATOR FCA UISPLACEMENT OH ACCELERATION SPECTMIM =

\begin{tabular}{|c|c|c|c|}
\hline $\begin{array}{l}E 0.0 \\
E O .1\end{array}$ & $\begin{array}{l}\text { D1SPLACEMEKT } \\
\text { ACCELEAATICN: }\end{array}$ & & . \\
\hline & & & . \\
\hline MODAL & PARTICIPATION & FACTORS & \\
\hline MODE & $X$ XIAECTION & Y-OINFCIJOA & Z-OIRECTION \\
\hline 1 & $.1752 E+0 n$ & $-.259 .3 \mathrm{~F}-\mathrm{nI}$ & $-. .3 .30 A E+0 \cap$ \\
\hline 2 & $.3 F 28 E+00$ & $-. ? 074 \mathrm{~F}-n 2$ & $.147 \mathrm{BF}+00$ \\
\hline 3 & $\because .5397 E-01$ & $-. P 5 A 2 E+n \cap$ & $.2793 E-01$ \\
\hline 4 & $. A 361 E-01$ & $-.52 A A F=01$ & $-.1174 E-01$ \\
\hline 5 & $\because 7975 E-01$ & $-6 \times n 5 E-n 1$ &.$- i\{15 E-0)$ \\
\hline
\end{tabular}

SFECTAUM TAPLE IIMPEFIAL VALLEY E.o. ELCENTAO 1940 SE

NUMAEA OF PCINTS: $=1$

SCALE FACTOF : :

\begin{tabular}{|c|c|c|}
\hline IAPUT & & SPECTHLN \\
\hline POINT & PEFION & VALLE \\
\hline$i$ & IAQAE-O? & $.145 \cap E+0.3$ \\
\hline 2 & - $P P G \cap E-01$ & $.3 H \cap \cap E+0.3$ \\
\hline 3 & . $500 \cap E-01$ & $.7750,0+03$ \\
\hline 4 & .7 IOOE-0I & $.7750 E+93$ \\
\hline 5 & $.9 \mid 00 E-01$ & $.440 .0 E+03$ \\
\hline 6 & $.1140 E+0 \pi$ & IIAPE 04 \\
\hline 7 & $.1410 E$ in & .11 DPE 04 \\
\hline Q & .172 OF A. On & $.7 \cap 0 \cap E+\cap 3$ \\
\hline 9 & ? OOOE $\triangle 00$ & $.071 D E+03$ \\
\hline 10 & $. ? 500 E+0 n$ & $.871 \cap E+03$ \\
\hline 11 & 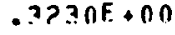 & $.4 \cap 0 \cap E+n 3$ \\
\hline
\end{tabular}




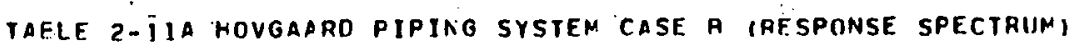
RESPOA SF. SFECTRUM CISPLACEMENT COMPONENTS

RESPONSE FCE MOCE
FREDUEACY $=$ TA.

CISPLACFMENTSAHITATIONS OF UARFSTRAINED AORFS

\begin{tabular}{|c|c|c|c|c|c|c|}
\hline $\begin{array}{l}\text { NONE } \\
\text { AUMHF.R }\end{array}$ & TRANSLATTON & ATION & $\begin{array}{l}\text { 7:- } \\
\text { RANSLATION }\end{array}$ & POTATION & FOTATION & ROTATIOA- \\
\hline 2 & B.7ก5フAE-ח4 & $1 . A B, 09 E-07$ & $6 E-0.3$ & $-7.1117 \mathrm{BE}-05$ & -2.5490 AE -0.5 & $-2,7 \overline{2} 979 \varepsilon-05$ \\
\hline 3 & $6696 E-03$ & -07 & 3 & 05 & GE -05 & $-3.19546 E-05$ \\
\hline 4 & & 04 & & & $4 \mathrm{AF}-05$ & $\begin{array}{l}31254 E-05 \\
31625 E-05\end{array}$ \\
\hline 5 & $6.31 E-0.3$ & $-7.3 \times 46,3 F-04$ & $-6 . \operatorname{ARB3}$ & -5.29948 & -9.33 .9 A $9 F-05$ & -1.31 \\
\hline 6 & $-\ln 3$ & -04 & -4 & -4. & $\begin{array}{l}\text { 74.04AE-05 } \\
8 \text { OP?TE-05 }\end{array}$ & $\begin{array}{l}-1.05731 E-05 \\
-A .16467 E-06\end{array}$ \\
\hline 7 & 3.5 & $\begin{array}{l}-1.11674 F-03 \\
-8.8 C 454 E-04\end{array}$ & $\begin{array}{l}075 E-0.3 \\
\text { i7) } E-04\end{array}$ & $\begin{array}{l}-4.1 \\
-3.17\end{array}$ & $-8.355 \times 2 E-05$ & $-4.00842 E-06$ \\
\hline 9 & $9.0832=E-04$ & $-2.17550 E-04$ & & & -4 & $-3.52452 E-06$ \\
\hline in & $P .4407 E E-04$ & $.32305 E-05$ & $9790 \mathrm{DE}-06$ & 12AG $3 E$ & $151 f-05$ & 06 \\
\hline
\end{tabular}

CJSPLACEMEATSARTATIOHS OF UNGESTHÁTNED AOLES

\begin{tabular}{|c|c|c|c|c|c|c|}
\hline $\begin{array}{l}\text { NODE. } \\
\text { NUMBER }\end{array}$ & TFANSLATION & THANSLATION & IRANSLATION & ROTATION & RGTATION & FoTATIOA \\
\hline 2 & 7.KATOSE-04 & $-1.7+02 \cap E-07$ & $6.12064 E-04$ & $1.72317 F-05$ & 1.1812AE-05 & $-2.15718 E-05$ \\
\hline 3 & 1.8745 P. -03 & $-3.51689 \mathrm{E}-07$ & $1.49613 E-03$ & $1.20391 E-05$ & $6256 E-05$ & -05 \\
\hline 4 & ?.0AOก9E -0.3 & $-E .01291 E-05$ & $1.3296 \mathrm{AE}-03$ & $1.42574 E-07$ & $1139 E-05$ & 06 \\
\hline 5 & 2.051 ? IE -0.3 & $-\overline{C A} 3319 \mathrm{~F}-05$ & 4.AR691F-104 & $5494 E-06$ & $A \cap E-05$ & 07 \\
\hline 6 & $2.0490 \in E-03$ & $-1.56707 E-05$ & $-1.56136 F-05$ & 07 & 5242AE -05 & $5.95008 E-07$ \\
\hline 7 & ?.04614 EE-0.3 & $-A . A S 32 H \mathrm{HE}-06$ & $-4.0 .3941 F-04$ & 4663 & $9172 E-05$ & 4.17 \\
\hline A & $1.93274 E-0.3$ & Z.A7 $105 E-0 \mathrm{~A}$ & -04 & -7 & 14t55E-05 & $3.39194 E-0 Q$ \\
\hline 9 & $1.01461 E-03$ & $3.7 \div 34 \mathrm{KE}-\cap \mathrm{K}$ & $2.51445 E-n B$ & $9.63131 E-08$ & $-3.73320 E-05$ & $3.86348 E-\cap 8$ \\
\hline 10 & G $4 R \in E$ & -06 & & & & , ne \\
\hline
\end{tabular}


TABLE ?-IIB HOVGAARD PIPING SYSTEM CASE A (RESPONSE SPECTRUM)

RF SPONSE SPECTRUM DISPLACEMENT COMPONENTS

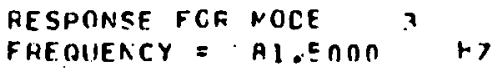

CISPLACEMENTS,FOTATIONS OF UNRESTIRAINEO NODES

\begin{tabular}{|c|c|c|c|c|c|c|}
\hline $\begin{array}{l}\text { NOIIE } \\
\text { UMBER }\end{array}$ & IRANSLATION & $\begin{array}{r}Y- \\
\text { THARSLAIION }\end{array}$ & TRANSLATION & ROTATION & $\begin{array}{r}\text { Y- } \\
\text { ACTATION }\end{array}$ & ROTATIOA \\
\hline 2 & $=4.4540$ PE -05 & $f .1=579 E-07$ & $2.29749 F-05$ & $4.9459 B F-07$ & -2.4267 & $0.62767 E-n 7$ \\
\hline 3 & E & 4.35 & $3090 E-05$ & & OR & .07 \\
\hline $\begin{array}{l}4 \\
5\end{array}$ & 3. & & & & $\begin{array}{l}07 \\
.09\end{array}$ & $E=06$ \\
\hline 6 & 05 & $65 F-04$ & -05 & -2 & -1 & 06 \\
\hline 7 & .05 & -04 & -05 & & 07 & \\
\hline B & 2 & -04 & & & 07 & .09 \\
\hline 9 & $\begin{array}{l}1.2628 Q E-05 \\
3.74766 E-06\end{array}$ & $\begin{array}{l}-8.5 \text {.501E } \\
-\bar{C} .55776 E\end{array}$ & $\begin{array}{l}04 A O E-08 \\
530 A E-0 A\end{array}$ & $\begin{array}{l}9996 E-06 \\
795 A E-06\end{array}$ & $\begin{array}{l}33 P 1 E-07 \\
0361 E-07\end{array}$ & $\begin{array}{l}1.24109 E-07 \\
6.20547 E-n B\end{array}$ \\
\hline
\end{tabular}

$\underset{\substack{1 \\ 1}}{+}$

RESPONSE FCR MOCE

FAEDUENCY $=141.747^{\circ} \quad 17$

DISPLACFNEATS,FOTATIONS OF UNRFSTRAINED NODES

\begin{tabular}{|c|c|c|c|c|c|c|}
\hline $\begin{array}{l}\text { NODE } \\
\text { RUMAER }\end{array}$ & TFANSLATION & TFAASLATION & TRANSLATIONO & $\begin{array}{r}x_{-} \\
\text {ROTATION }\end{array}$ & ROTAIION & Potation \\
\hline خ & $.29595 E-06$ & $7496 \mathrm{E}-\mathrm{n} 7$ & $-5.25629 E-05$ & $-7 . A 3 B 11 E$ & $-5.5 A 02 . A E-\cap 7$ & $-A .2 B 3 A 4 E-08$ \\
\hline $\begin{array}{l}3 \\
4\end{array}$ & & & & & & \\
\hline 5 & 06 & -7.8 & $485 B 9$ & 6 & -07 & $\begin{array}{l}-07 \\
-07\end{array}$ \\
\hline 6 & & $\begin{array}{l}-1 . \\
-1 .\end{array}$ & & & $\begin{array}{l}-07 \\
-06 \\
-07\end{array}$ & $\begin{array}{l}07 \\
07 \\
07\end{array}$ \\
\hline 10 & $\begin{array}{l}2.21504 E-05 \\
2.33499 E-05 \\
\text { P.60520E-06 }\end{array}$ & $\begin{array}{l}-1.8594 \mathrm{BE}-05 \\
-1.2054 \mathrm{AE}-105 \\
-4.21130 \mathrm{O}-0 \mathrm{~A}\end{array}$ & $\begin{array}{l}4.57410 E-n 6 \\
4.62335 F-n 7 \\
2.3) 3 A P F-n 7\end{array}$ & $\begin{array}{r}2.01256 E-01 \\
-3 . A 2 A O D E-07 \\
-3.1 \text { A } 26 E-017\end{array}$ & $\begin{array}{l}9.09747 E-117 \\
-6.16306 E-07 \\
-6.26747 E-07\end{array}$ & $\begin{array}{l}2.14791 E \\
1.07396 E\end{array}$ \\
\hline
\end{tabular}


TADLE 2-IIC HOVGaARD PIPING SYSTEM CASE h (AESPONSE SPECTRUM) AESPONSE SPECIRUM CISPLACEMENT COMPONENTS

RESPONSE FCA MOCE 5
FAEOUFACY $=168$. A?O

CISPLACEMFNTS,HOIATIONS OF UNAESTAAINED AOERS

\begin{tabular}{|c|c|c|c|c|c|c|}
\hline $\begin{array}{l}\text { NONEE } \\
\text { NUMAER }\end{array}$ & TRANSLA IOSN & TFAMSLATION & THANSLATION & $\begin{array}{r}x- \\
\text { HOIATION }\end{array}$ & RCTATION- & POTATIOA \\
\hline ? & $-4.55 \cap 9 E E-\cap 5$ & 2.5P51 2E-OR & $-3.615225-06$ & -3.731 PE-OB & $7.69919 E-D A$ & $5 . R 25 A B E=07$ \\
\hline 3 & $573 E-0.5$ & $5.12435 E-\cap A$ & -2.3TATIE-กT & $1.50907 E-07$ & 1.E3964F-07 & $-1.22149 E-06$ \\
\hline 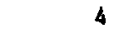 & $1.59407 E-05$ & $-1.49824 E-05$ & $3.79472 E-06$ & $335 F-07$ & BE -07 & $3 E-n E$ \\
\hline 5 & ?.09431E-05 & $-1.67287 E-05$ & $2.1585 \pi f-0 h$ & $.7 .91530 E-07$ & $1.95466 \mathrm{GE}-07$ & P. $24004 E-07$ \\
\hline 6 & $2.09440 E-05$ & I. . .94 PAE - OS & $-1.23321 E-06$ & $4.56585 E-07$ & $1.7 D E A \triangle E-07$ & $1.16218 E-n 6$ \\
\hline 7 & $2.10060 E-05$ & $2.41309 E-0.5$ & $-3 . A 55 \mathrm{I} .3 \mathrm{E}-06$ & $5.21639 E-07$ & $11703 E-07$ & $49 E-06$ \\
\hline A & $2.01308 \mathrm{E}-05$ & $4.43+59 E-05$ & $-3.17483 E-06$ & $7.65 .3378-07$ & -07 & -07 \\
\hline 9 & $1.15 ?$ & -05 & -19 & $9.50000 E-07$ & $-3.9125 \mathrm{NE}-07$ & $3.78726 E-07$ \\
\hline 10 & P1167te-06 & $.6 P G Q Z E-06$ & $4.23311 E=09$ & $.95 n 06 t-0.7$ & 895 KnE -0. & $1.49363 k=01$ \\
\hline
\end{tabular}




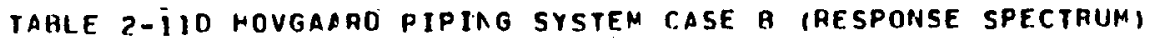

Response Spectrum Displacement Components

SOHARE ROOT OF IIF SUM GF THE SBUAFES OF THE NOUAL DEFLECTIONS

DISPLACEMFNTSIFOTATIGNS OF UNRESTRAJNED NODES

\begin{tabular}{|c|c|c|c|c|c|c|}
\hline $\begin{array}{l}\text { NODE } \\
\text { IJMAER }\end{array}$ & TFANSLATION & TFAASLA $Y$ ION & TRANSLATION & ROTATION & RCTATION & FOTATIOA \\
\hline 2 & 1.1617 PE-03 & $6.6+3 P O E-07$ & $2.33544 E-03$ & $7.31807 E-05$ & $810 B 7 E-05$ & $3.48104 E=0$ \\
\hline 3 & $3.19537 E-03$ & $1.3=98 \mid E-06$ & 6. & 5 & 05 & 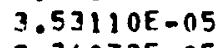 \\
\hline 4 & $3.2964 J E-03$ & 2. $.4754 i \equiv-04$ & $\theta$ & 6. & 05 & 4 \\
\hline 5 & $4.07107 E-03$ & $1.45424 \bar{E}-04$ & & 5 & & \\
\hline 6 & $4.06965 E-03$ & S. SE $366 E-04$ & $2 F=0 . ?$ & $E-05$ & ?. $1 E-04$ & \\
\hline $\begin{array}{l}7 \\
8\end{array}$ & $\begin{array}{l}4.067 A E E-0.3 \\
3.19 ? 74 E-03\end{array}$ & $\begin{array}{l}i .1375 \text { AE-03 } \\
8.0267 C E-04\end{array}$ & $\begin{array}{l}3.21653 E-03 \\
e .40526 E-04\end{array}$ & $\begin{array}{l}4.16904 E-05 \\
3.20176 E=05\end{array}$ & $\begin{array}{l}\text { O2 A OE }-04 \\
\text { A } 343 \mathrm{JE}-05\end{array}$ & $\begin{array}{l}A .48 \\
4.08\end{array}$ \\
\hline 9 & ].3G? IOE -0.3 & 3. उC2A=E. -04 & $4.218 A 4 F-06$ & $1.51495 E-05$ & 5465 PE - 05 & 06 \\
\hline in & $3.99709 E=04$ & P.7711 $\times E-05$ & $2.10960 F-05$ & A. $16288 E-06$ & $3.44615 E-05$ & 7684 \\
\hline
\end{tabular}


TAGLE 2-12 hovgadRo PIPING SYSTEM CASE a (AESPONSE SPECTRUM)

SGUARE HOOT OF THE SLP OF THE SGUARES OF THE MDDAL FORCES (FOH ALL FLENENTS)

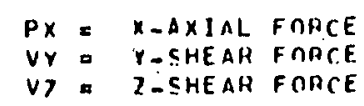

IX $=X$-TKISTING MONEAT

MY $=Y$ - EENOINE MONERT

Y - SHEAR FARCE.

$M Z=$ Z - PENDINE MONEAT

$I=$ INITIAL NONE

$C=$ CFNTER RODE IFOR PIPE BEAD ONLY

$J=$ FINAL NOUE

element trae izic P I P E

$i, 1$, ELENENT NIJMBER 11

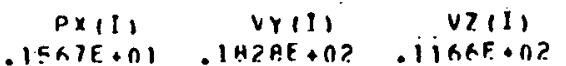
IXII) MYII) MZII)

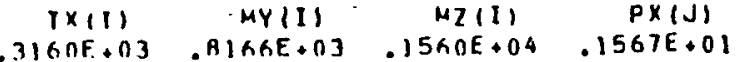
WXII) VYII) VZII) IXIII MYIII MZ(I) PXI

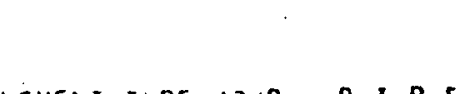

ELEMEAI TYPF IJ/C P I P F.

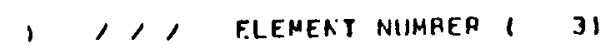

IX(1) MY(I) MZ(I) PXIC) $\begin{array}{ccc}M 160 F .03 & .2559 E+03 & .2644 E+03\end{array}$

VY (J)

$\operatorname{vin}(3)$

.5
$1 \times(7)$
$.31600+03$

HY(J)
$.2644 E+03$

T59E.03 $P \times(C)$
$3006 E+01$ $V Y(C)$
$57: 3 ? E+0$ $v 2(C)$
$.5626 E+01$

ELEMEAT TYPF I3/C P I P E

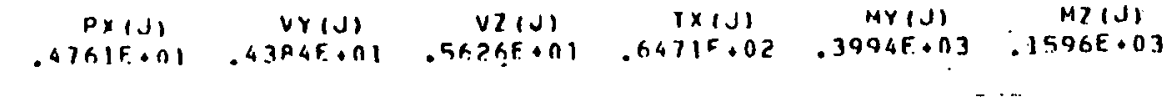

elemeat typF $13 / \mathrm{C}$ P I PE

1,1, FLEMENT NIIMBEA 141

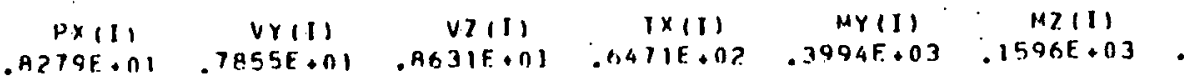

$\operatorname{Px}(\mathrm{C})$

VY(C)

$v z(c)$

$T \times(C)$

$\operatorname{Mr}(C)$

$M Z(C)$

ELENEAT TYPE 13/0 F I P F.

1

ELENEAT NIIMHER

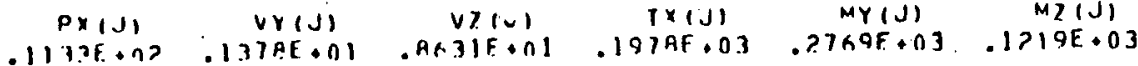

ELEREA TYPF IJ/R F 1 P E

1,1, ELENENT NIIMAER. 151

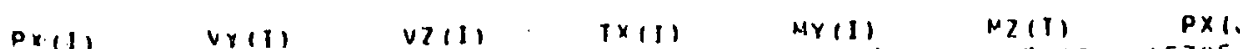

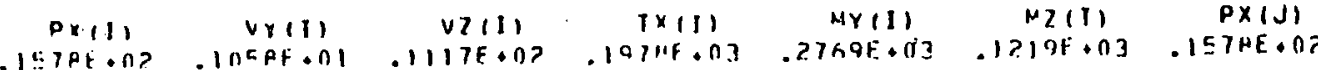




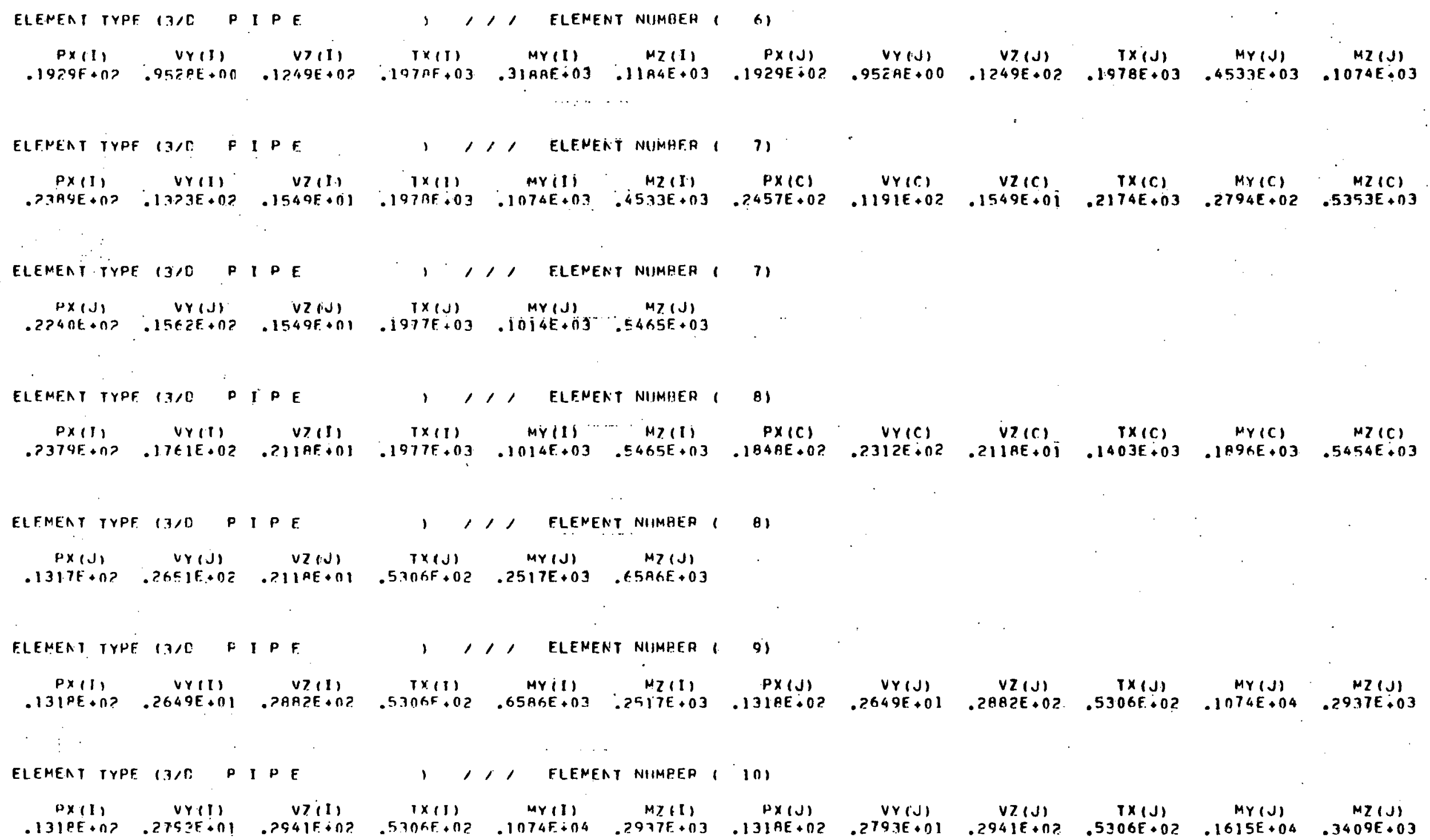


TARIE 2-13 MOVGAARO PIÖHG SYSTEN CASE O IAESPONSE SPECTRUM, RF SPONSE SPECTRUM STRESS COMPONENTS

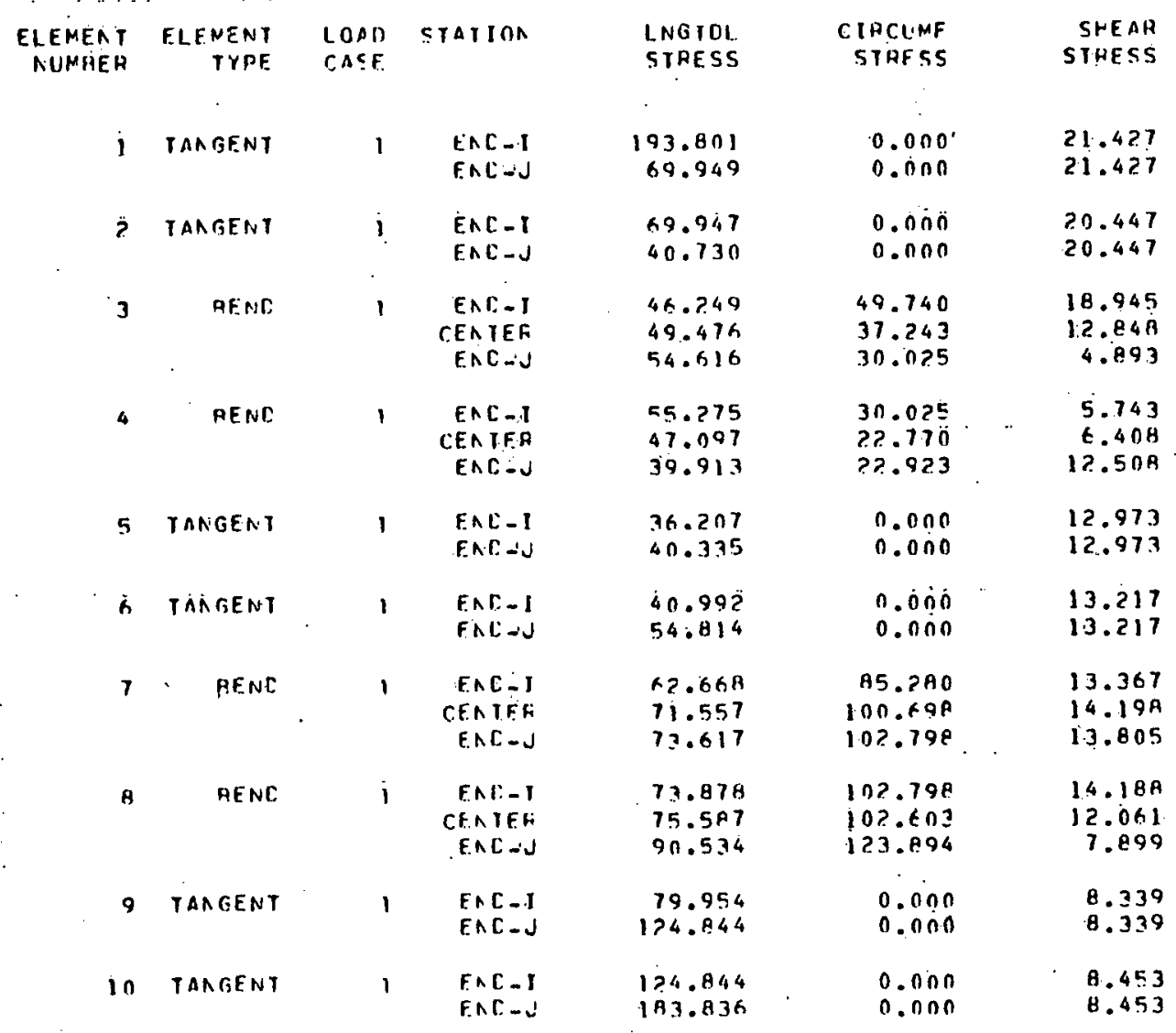




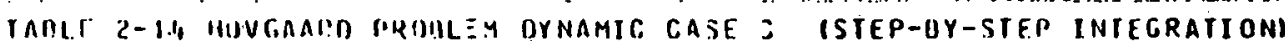

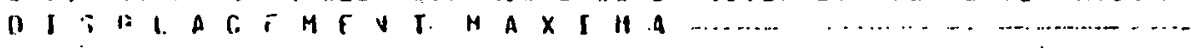

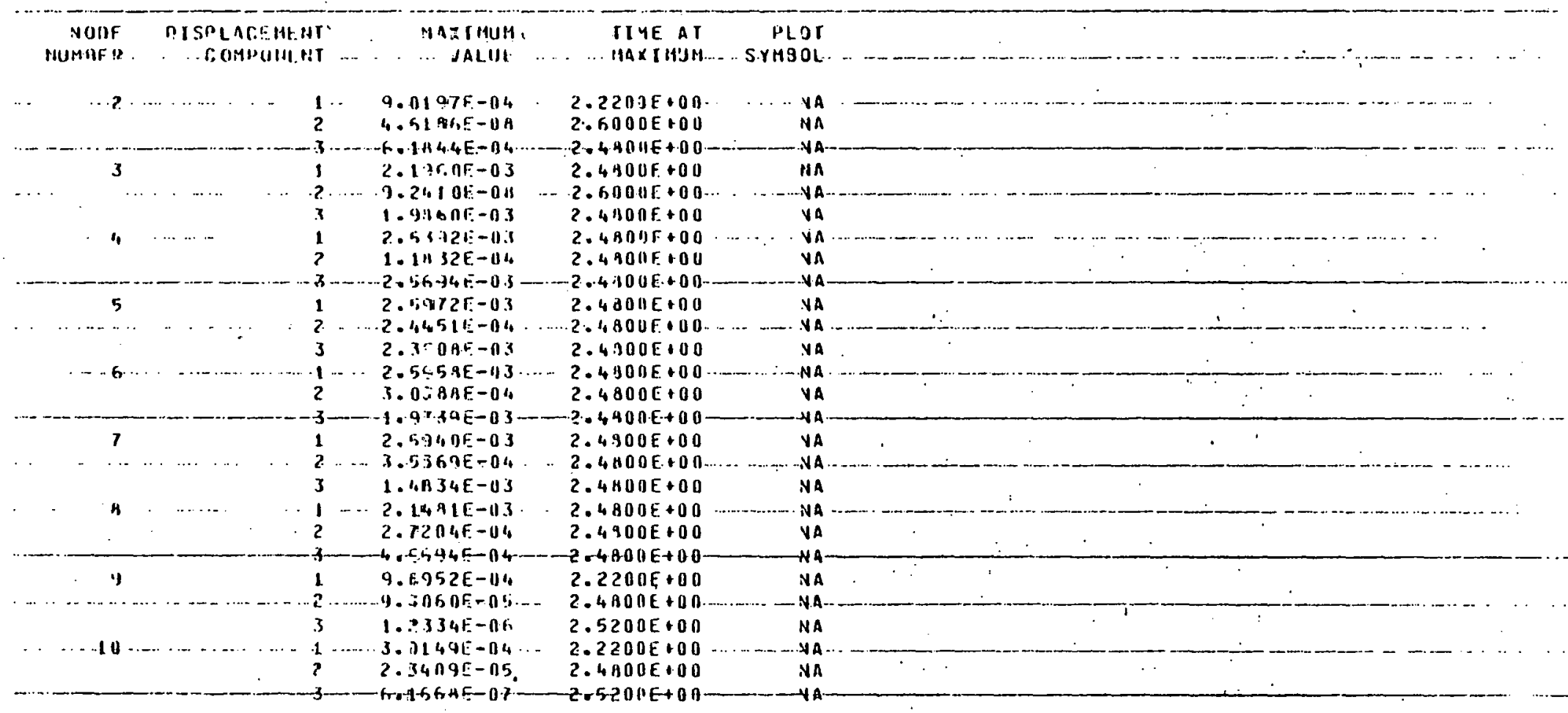

Note: Displacement components 1,2 , and 3 respectively correspond to $x, y$, and $z$. 


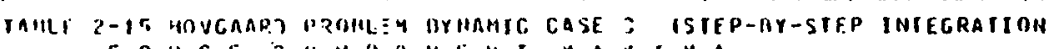

$$
\text { FOUCF-O }
$$

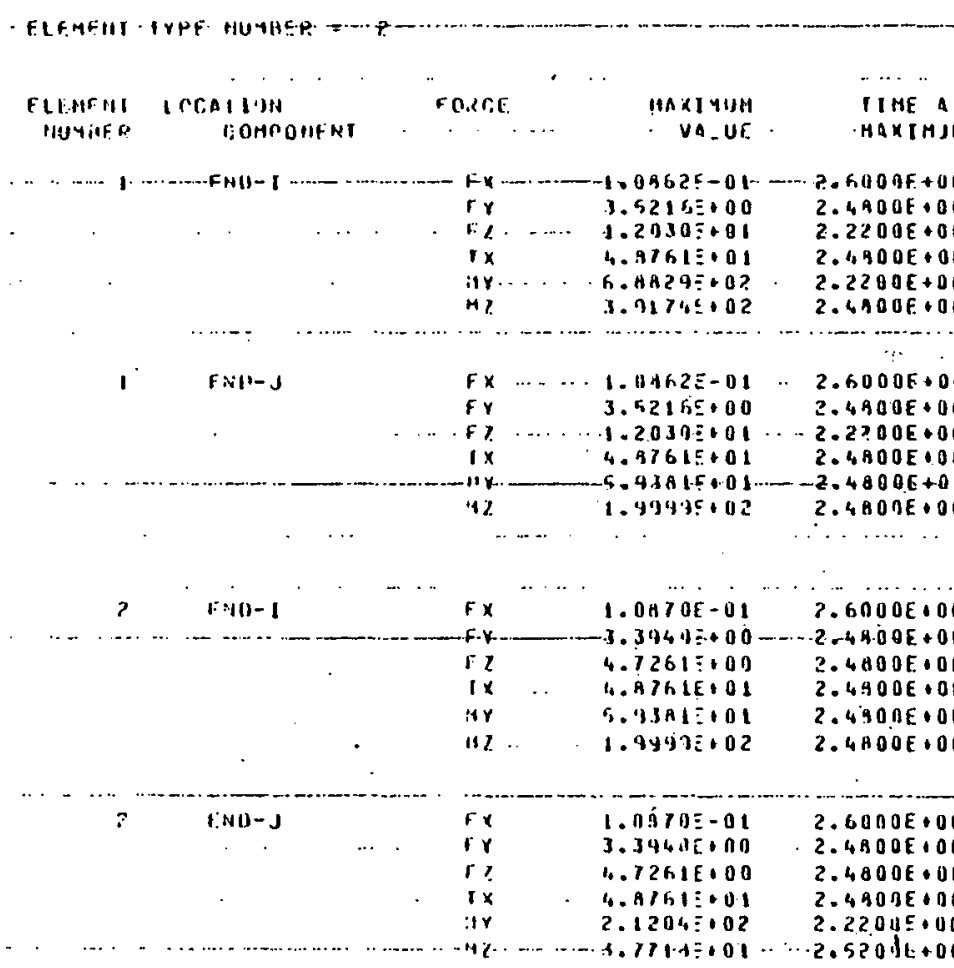

I rasi-I

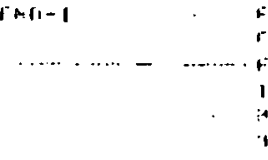

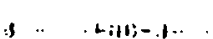

$x x$
$r y$
$y$
$1 x$
$i+y$
11

P. 0A4?:-0 $4.51155=010$ $2.41945+011$ $3.1714:+0$ 2.12045020

\begin{tabular}{|c|c|}
\hline & \\
\hline$r_{Y}$ & $1.116 .4,10: 00$ \\
\hline , & $2,9.8+1=0$ \\
\hline Ix & 1.3121600 \\
\hline "rr & 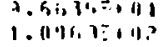 \\
\hline
\end{tabular}

$2 \cdot 60005+90$

2.4nORF:00

2. $4.40,0.000$

$2.5200 E 000$
$2.2=006000$

\section{2?000000}

2.220115110

$2.430 .1 \mathrm{f} .0 \mathrm{OA}$

?.4ARBE I gU

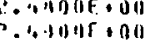




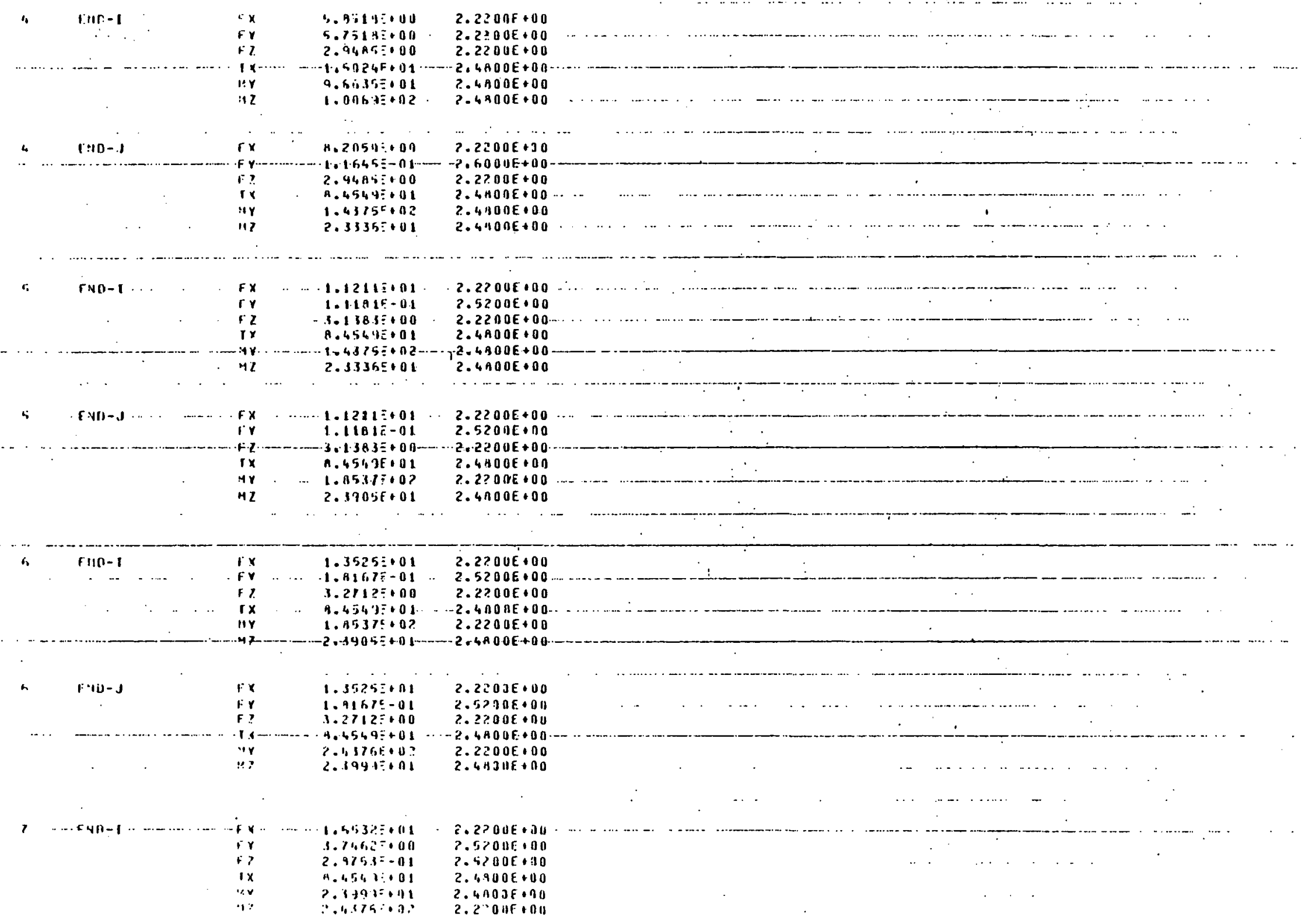




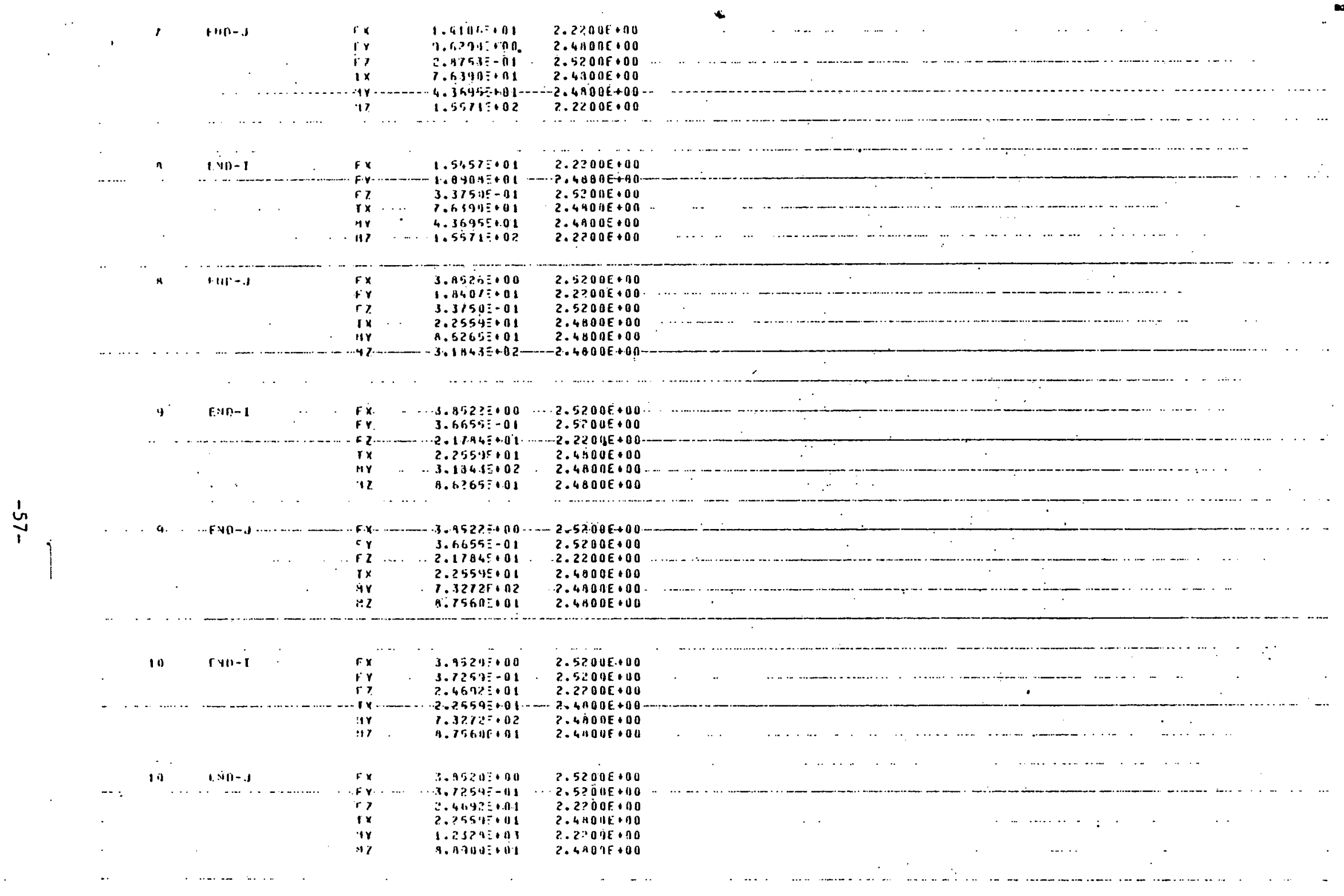




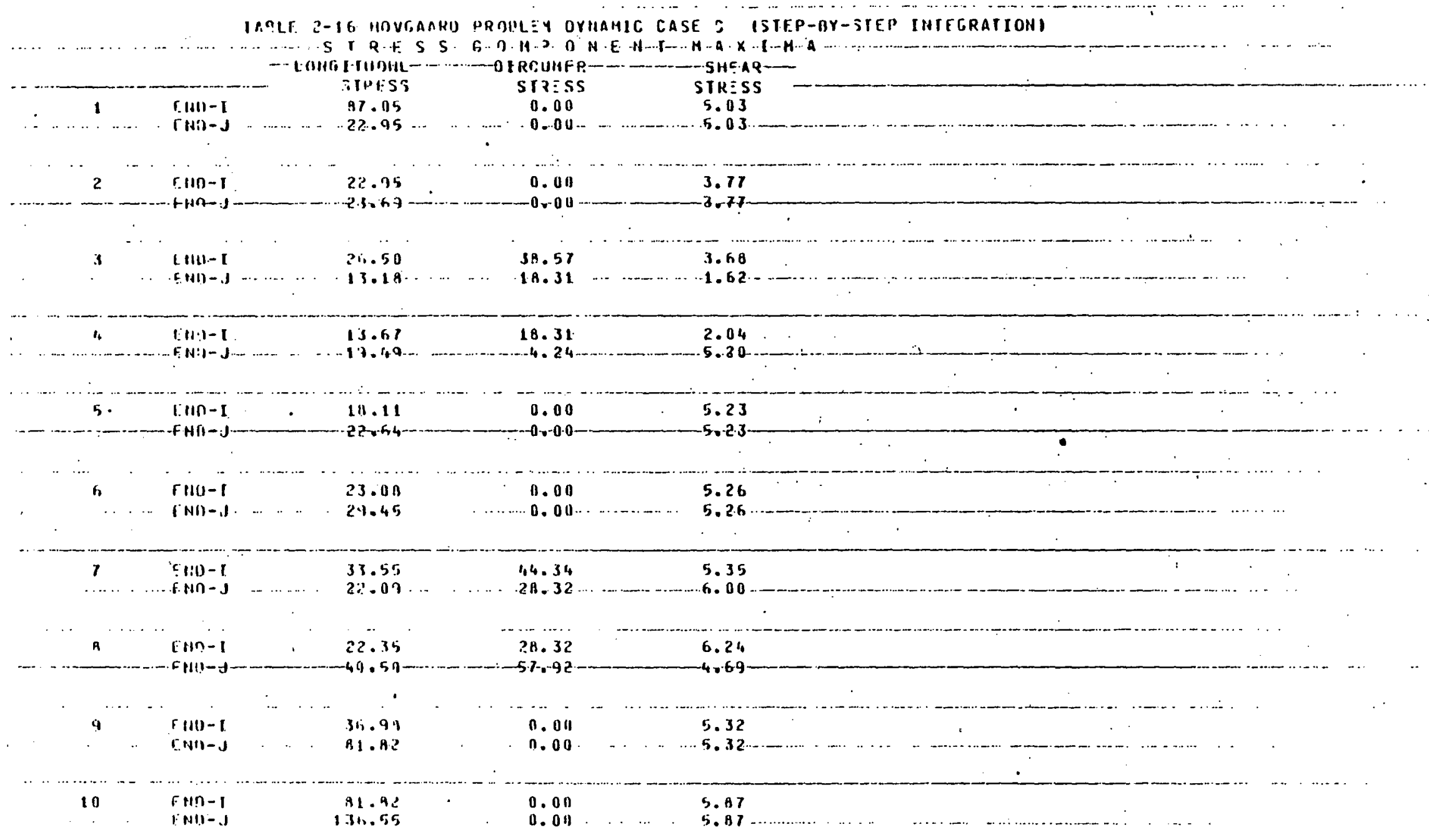




\section{Problem 3 - Dynamic Analysis of a Coffee Table}

The third problem to be investigated was the dynamic response of a coffee table subjected to the identical earthquake motion discussed previously for Problem 2. Results gotten for the three different methods of analysis are given in the tables that follow shortly.

\section{Problem Description}

i. Geometry - The configuration is as shown in Fig. 3-1a. The structure is comprised of lengths of 2 in. steel pipes with internal lining of damping material, and four $23 / 4$ in. steel cubes welded with the pipes at the corners as shown in the figure. The entire assembly is fixed to a heavy base. The lumped mass system used for the analysis is shown in Fig. 3-1b. The system is the same as the one used by swanson ${ }^{16}$, in which the mass of the cube plus three adjacent $3-5 / 8$ in. lengths of the piping were lumped at each corner. Intermediate mass points $M_{2}$ and $M_{1}$ respectively, comprising the masses of 10 in. and 5 in. segments of the piping were positioned as shown in the figure. The horizontal dimensions of the pipes are considered only between the outside edges of two adjacent cubes. The input nodal coordinates and the values used for the lumped masses are shown in Table $3-1$ and Table $3-2$, respectively.

ii. Material data - The member properties are as follows: 


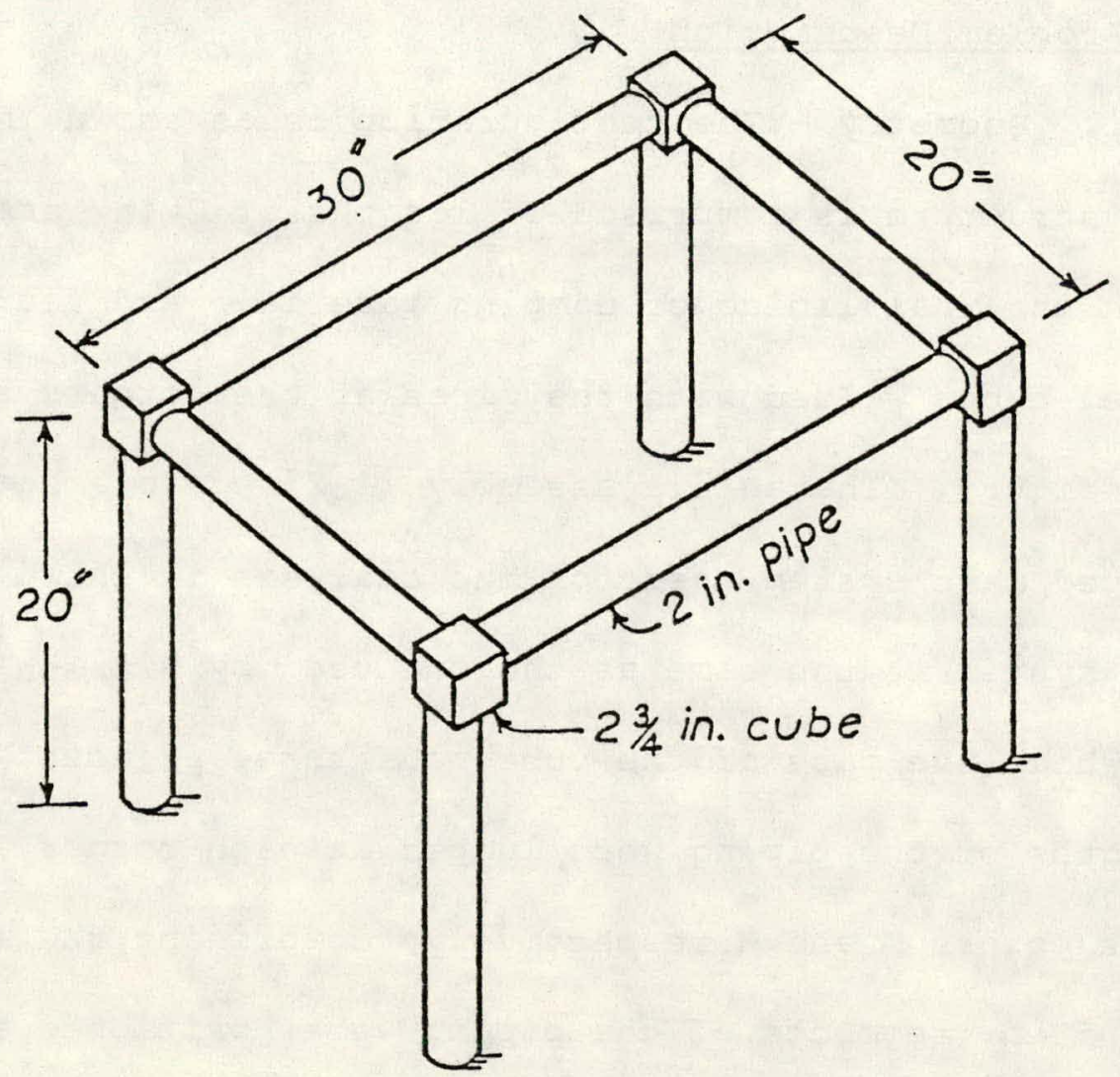

FIGURE 3-la

ISOMETRIC SKETCH OF COFFEE TABLE 

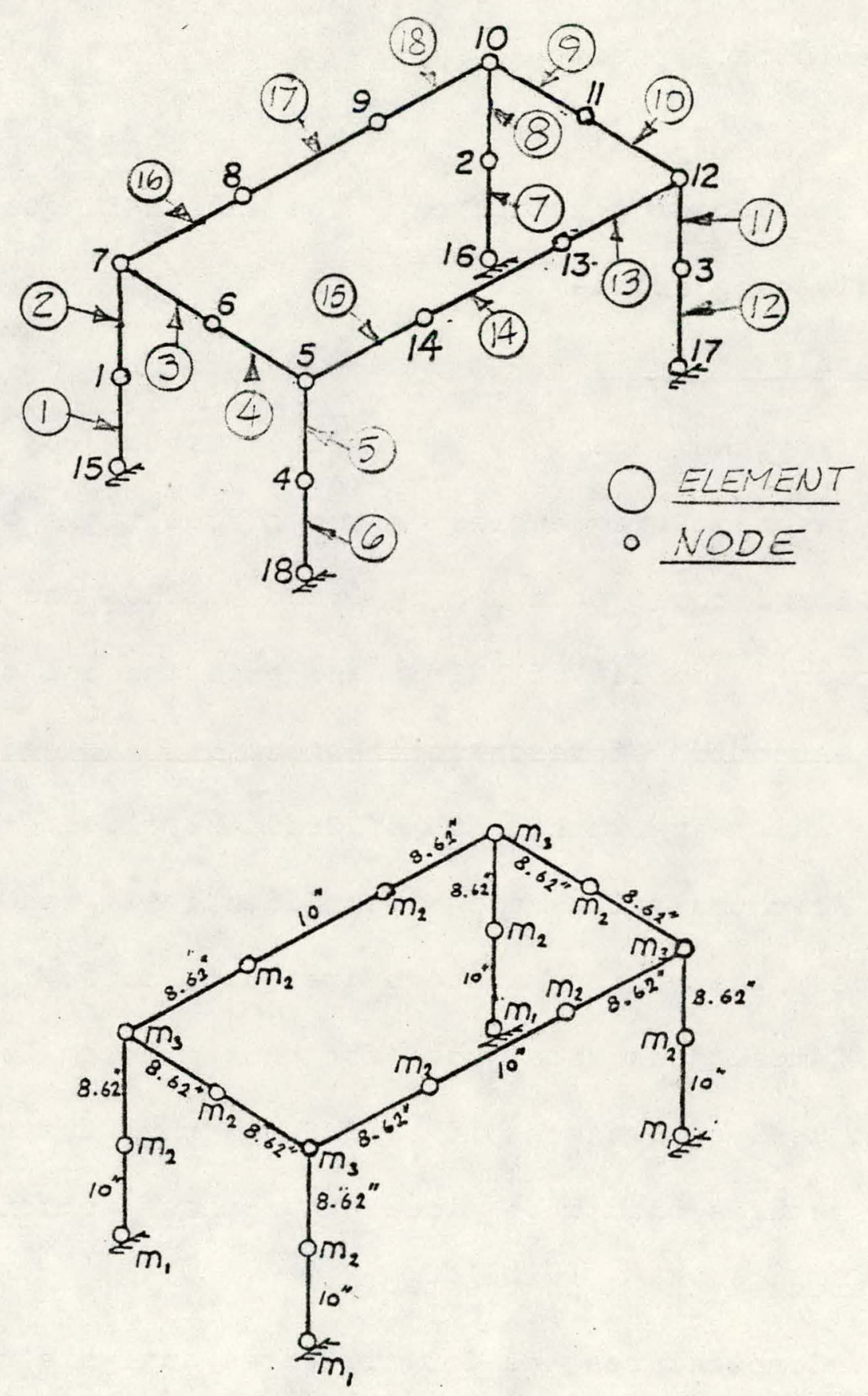

FIGURE 3-1b

FINITE ELEMENT MODEL AND LUMPED NASS

LOCATIONS FOR COFFEE TABLE PROBLEM 


$\begin{array}{ll}\text { Pipe outside diameter (inches) } & 2.375 \\ \text { Pipe wall thickness (inches) } & 0.154 \\ \text { Young's modulus (psi) } & 27.9 \pm 6 \\ \text { Poisson's ratio } & 0.3 \\ \text { Unit weight (1b per in. }{ }^{3} \text { ) } & 0.34517\end{array}$

iii. Loading condition - The same earthquake response as given in Problem 2 .

\section{Analyses Results}

For the purpose of comparison Table $3-3 a$ shows values for resonant frequencies of the coffee table obtained from EPIPE and those obtained by Crede ${ }^{15}$, Tuba and Wright ${ }^{17}$, Swanson $^{16}$ (by use of ANSYS) and experimental means. ${ }^{15}$ With the exception of resonant frequencies obtained for the 5 th mode which are within $13 \%$ of Crede's values, $5 \%$ of Tuba and Wright's values and almost identical with Swanson's values, the various answers are quite close. As mentioned, the lumped mass model used for this problem is the same as that used by Swanson with ANSYS and thus the results of the two methods should be closer than those gotten by the other references shown in the table.

Computer results for all three analysis cases are shown in Tables $3-3$ to $3-14$. Tables $3-3$ to $3-7$ respectively 1 ist the 
natural frequencies, mode shapes, maximum displacements, resultant forces, stresses, and their time of occurrence obtained from the time-history analysis. Similarly, tables 3-8 and 3-9 show the modal participation factors, displacments and relations, and the square root of the sum of the squares, or root mean square (RMS), of the modal forces and stresses obtained from the response spectrum analysis. Finally, the maximum displacements, forces and stresses and the time of occurrence from the step-by-step integration solution are given in Tables 3-10 to 3-14. Again, for this problem the numerical results from all three methods appear to be similar with the response spectrum analysis yielding generally higher resultant forces and stresses. For this problem, the time span was 16 seconds with 800 integration steps of 0.02 seconds each. Unlike the Hovcaara problem, only every fifth ingegration step was printea. 
TABLF ?-I INPUI DATA FOR COFFEF TAHLE PRORLFM NODAL C. OTRRDINATES

$+$

\begin{tabular}{|c|c|c|c|c|c|c|c|c|c|c|c|}
\hline \multirow{2}{*}{$\begin{array}{l}\text { NONE } \\
\text { NUMB ER }\end{array}$} & \multicolumn{2}{|c|}{ HCUNAARY } & \multicolumn{2}{|c|}{ CONEDITION } & \multicolumn{2}{|c|}{ COIISES } & \multicolumn{4}{|c|}{ NODAL POINT COORDINAIES } & \multirow[b]{2}{*}{$\mathbf{r}$} \\
\hline & $x$ & $\mathbf{v}$ & 2 & $x x$ & YY & 22 & $x$ & 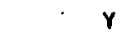 & I & & \\
\hline 1 & o & $\mathbf{0}$ & $\mathbf{0}$ & n & n & $\underline{n}$ & 0.0 & 10.000 & 0.0 & $\Omega$ & 0.0 \\
\hline 2 & 0 & 0 & 0 & 1) & n & 0 & 27.2 .50 & 10.000 & 0.0 & 0 & 0.0 \\
\hline 3 & $\mathbf{0}$ & $\mathbf{u}$ & n & u & 0 & 0 & 27.250 & 10.000 & 11.250 & 0 & 0.0 \\
\hline 1 & 0 & $n$ & 0 & 0 & (1) & n & -2.2 & 10.002 & 11.250 & $a$ & 0.0 \\
\hline 5 & 0 & 0 & 0 & 0 & 0 & 0 & 0.0 & 18.025 & 17.250 & $n$ & 0.0 \\
\hline 6 & 0 & $\mathbf{0}$ & 0 & 0 & 0 & 0 & $0: 0$ & 10.6 .25 & P. 625 & 0 & 0.0 \\
\hline 1 & 0 & i & 0 & n & n & 0 & 0.0 & in.625 & L.u. & 0 & 0.0 \\
\hline 11 & 0 & 0 & 0 & 10 & 1) & 0 & 0.625 & 18.625 & 0.0 & 0 & 0.0 \\
\hline 9 & o & 0 & 0 & () & n & 0 & 113.1 .25 & $14.6,25$ & 0.0 & 0 & 0.0 \\
\hline 10 & 0 & 0 & 0 & II & $\underline{0}$ & n & 21.250 & 16.625 & 2.0 & 0 & 0.0 \\
\hline II & 0 & 0 & 0 & 0 & 0 & 0 & 27.250 & 18.625 & 18.625 & 0 & 0.0 \\
\hline 12 & n & 0 & 0 & 0 & 0 & 0 & 27.250 & 18.625 & 17.250 & 0 & 0.0 \\
\hline 13 & 0 & 0 & 0 & 0 & 0 & e & $10 . \times 25$ & 10.625 & 17.250 & 0. & 0.0 \\
\hline 14 & 0 & 0 & 0 & 0 & 0 & 0 & A. 625 & 18.625 & 17.250 & 0 & 0.0 \\
\hline 1.5 & I & I & 1 & 1 & 1 & 1 & 0.0 & 0.0 & 0.0 & 0 & 0.0 \\
\hline 16 & 1 & 1 & $i$ & 1 & 1 & $i$ & 27.250 & 0.0 & Q1) & 0 & 0.0 \\
\hline 17 & .1 & I & 1 & i & I & 1 & 27.250 & 0.0 & 17.250 & 0 & 0.0 \\
\hline 18 & I & 1 & 1 & 1 & 1 & 1 & 0.0 & 0.0 & 17.250 & o & 0.0 \\
\hline
\end{tabular}

! 
IAAI + 3-? IMPUT DAYA fOK COFfEF TARLE PRDBLEM

L UH PED MASSFS DATA

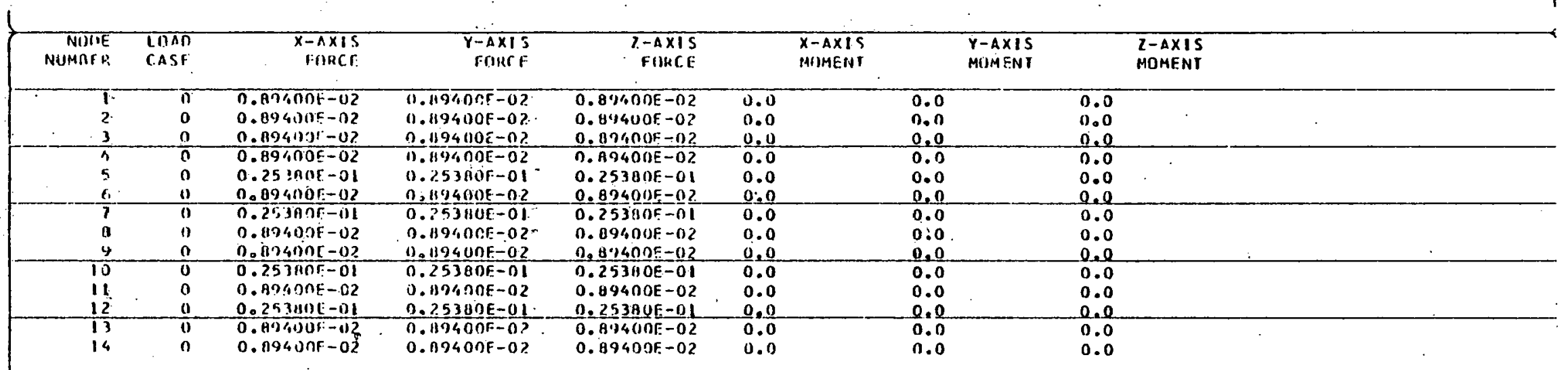




\section{TABLE $3-3 a$}

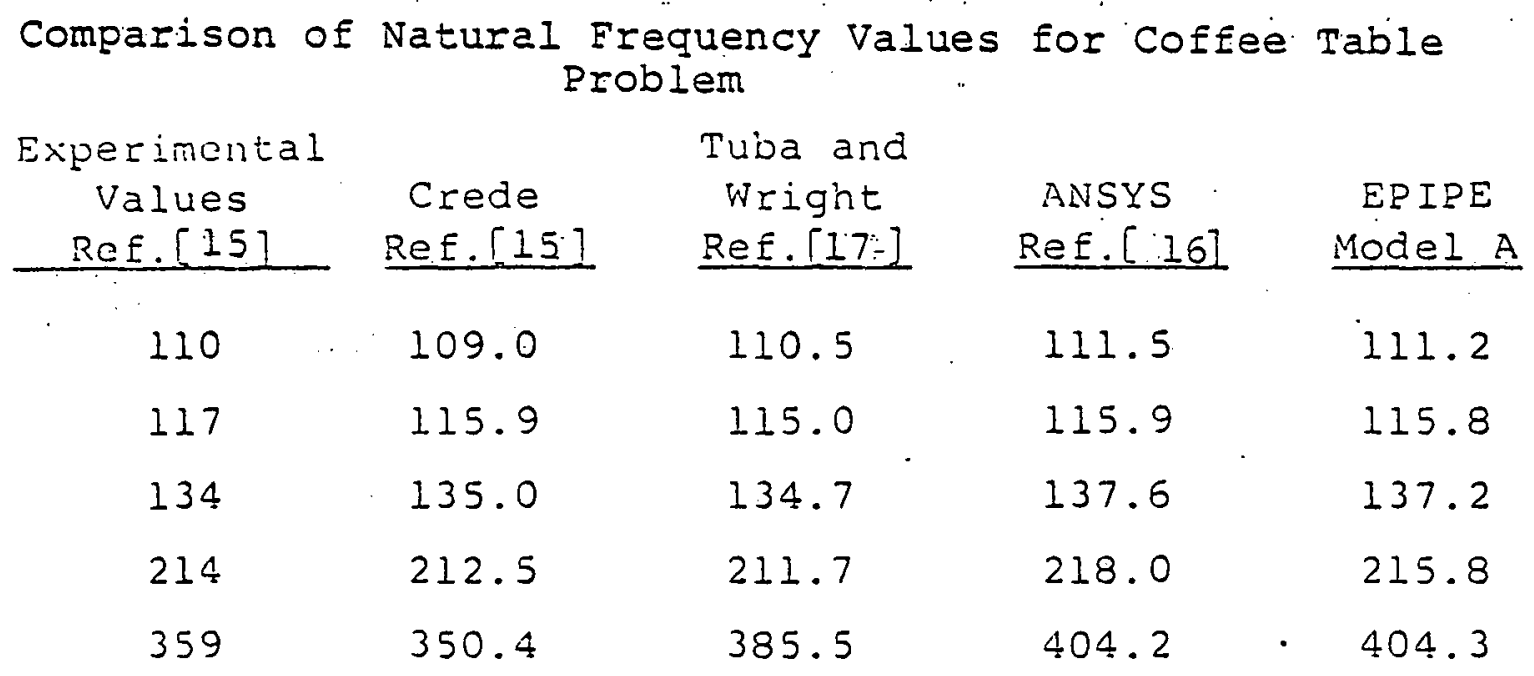




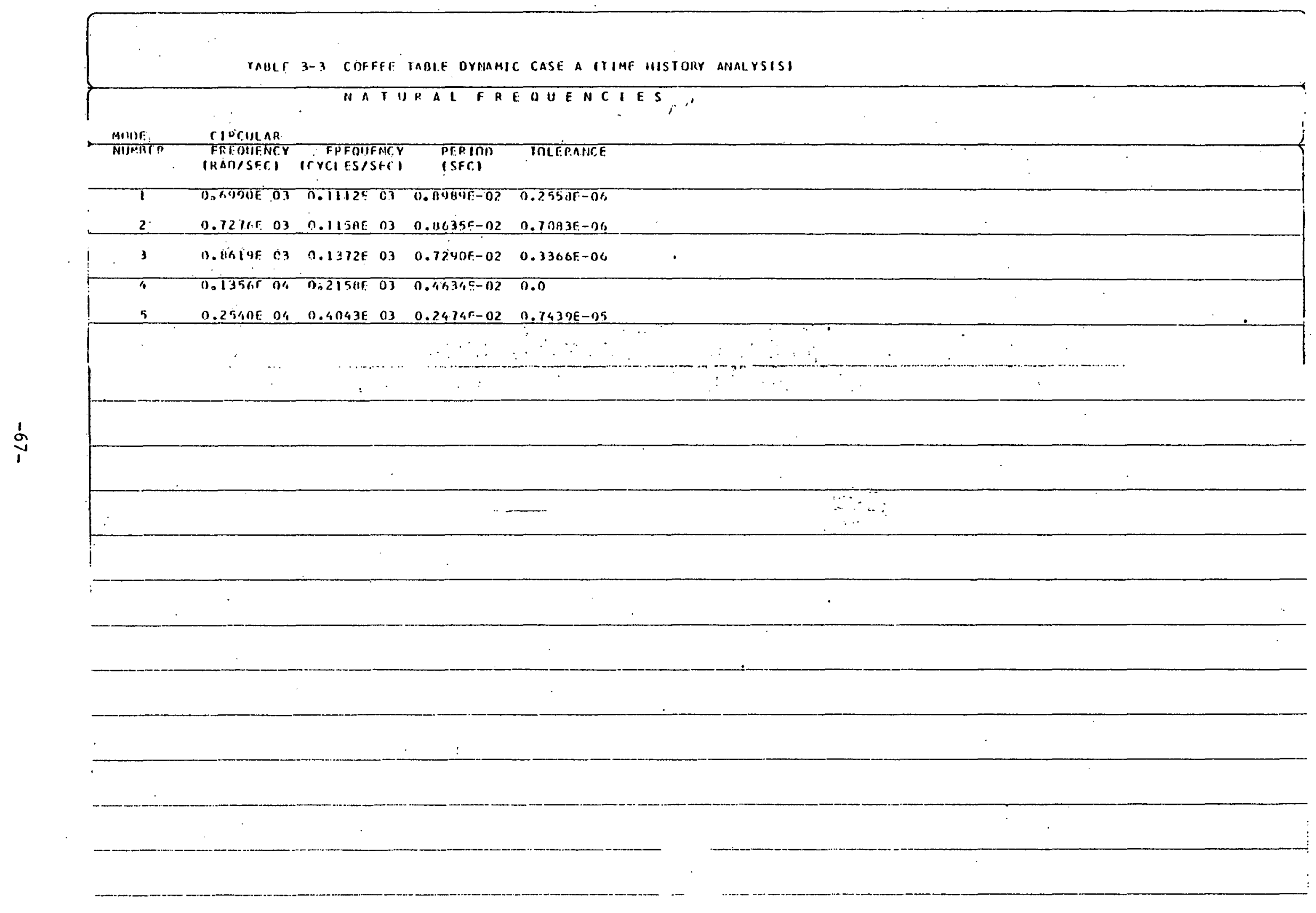




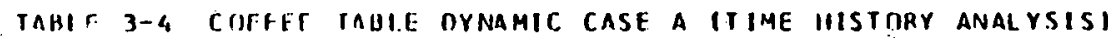

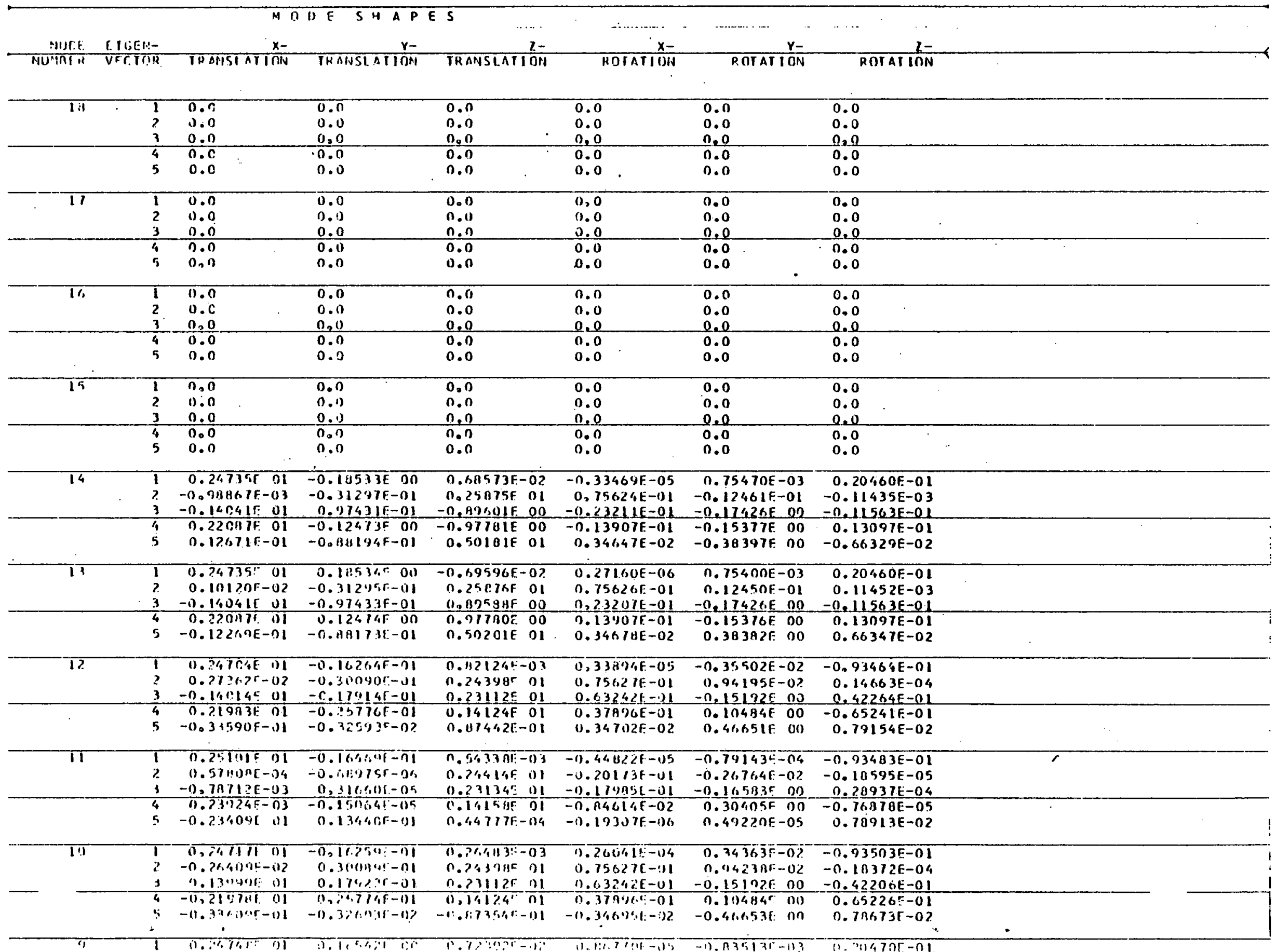




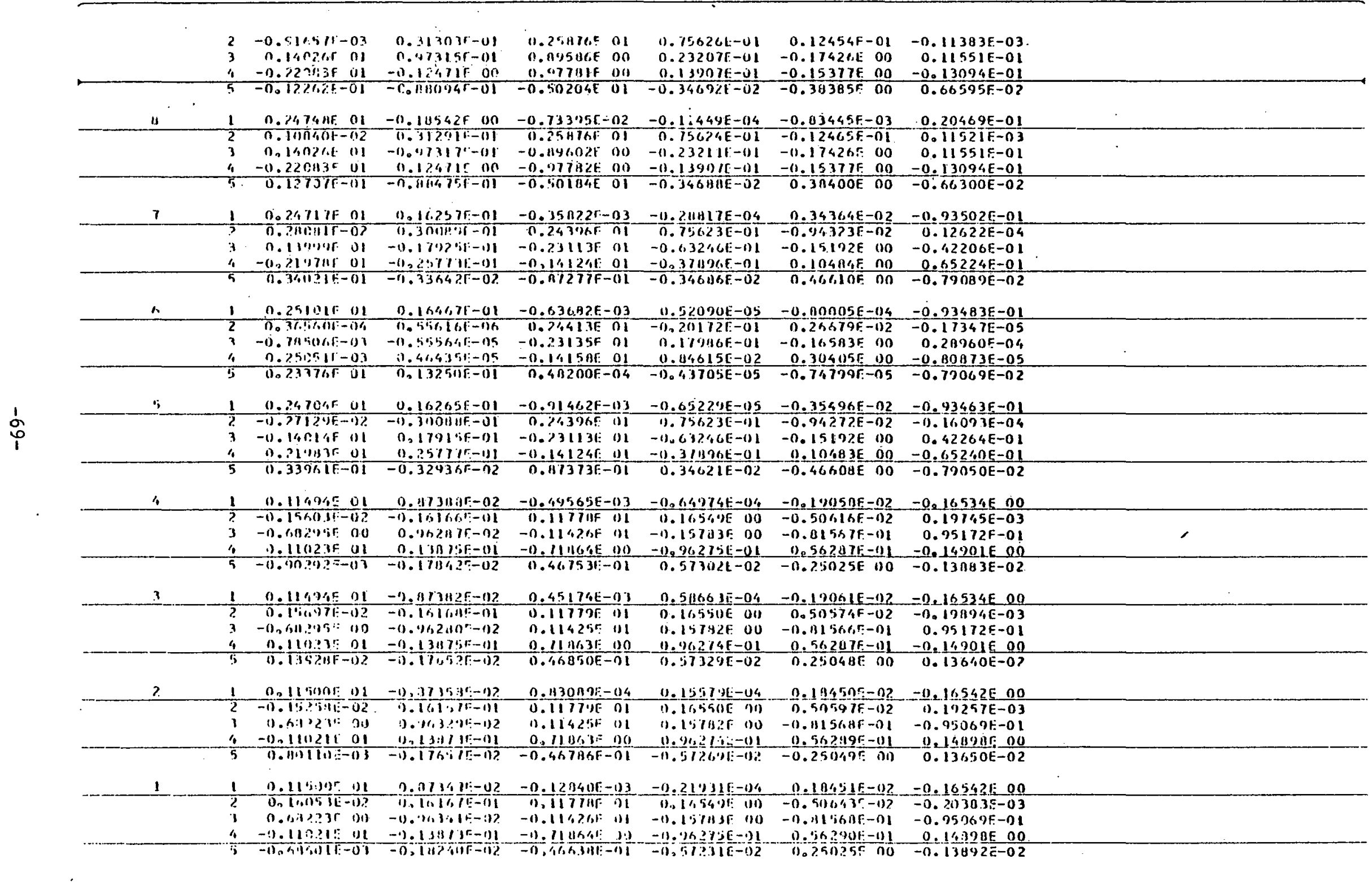

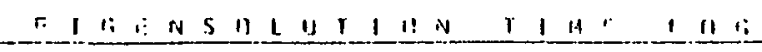

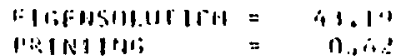


TABLF. 3-5 CrIfFer table DYNAMIC CASE a ITIME HISTORY anAI.YSISI

D I S P I. A C E HENT MAXIMA _..

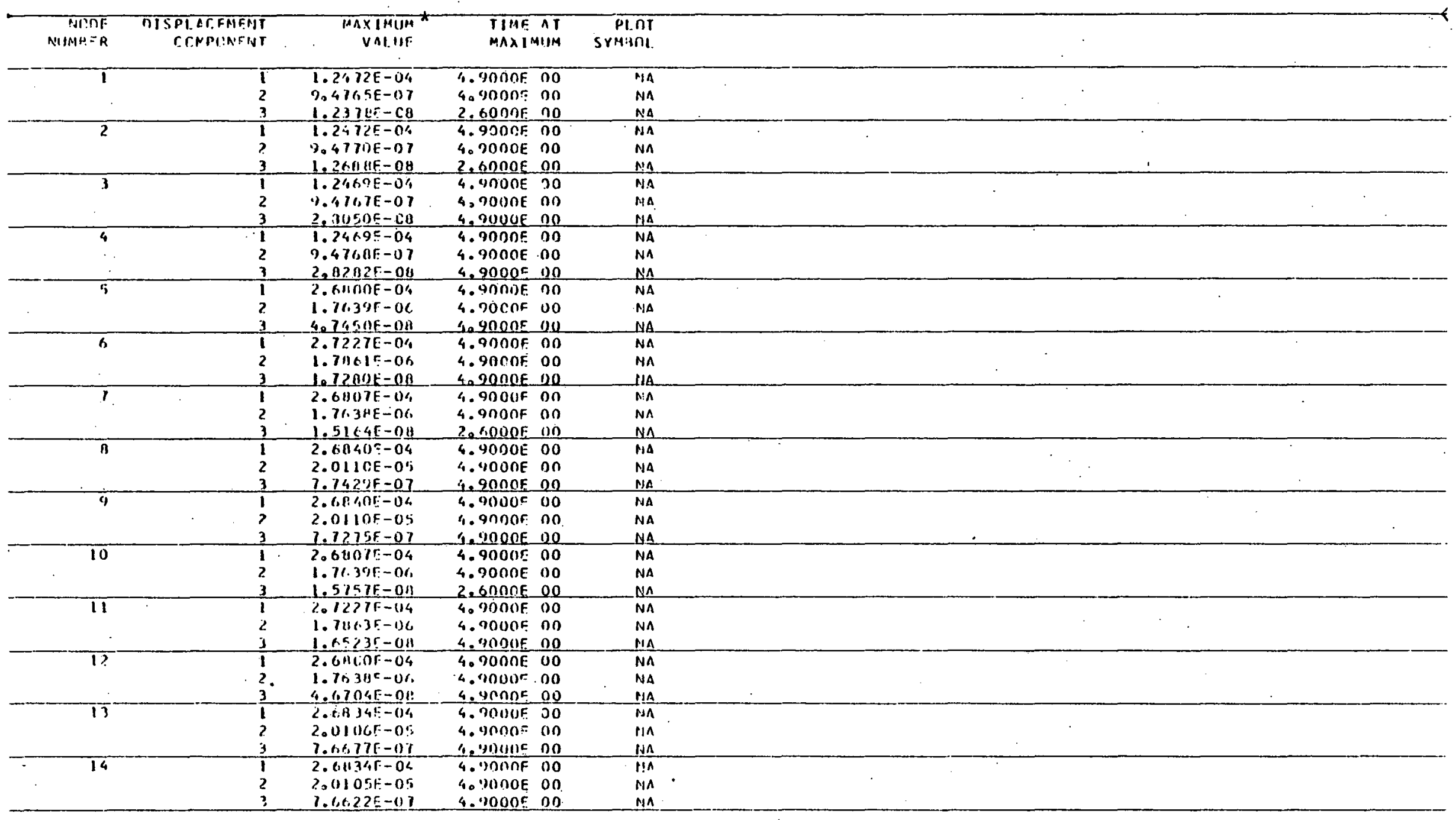

*A1l values represent the absolute maximum

Note: Displacement Components 1,2 , and 3 respectively correspond to $x, y$, and : 


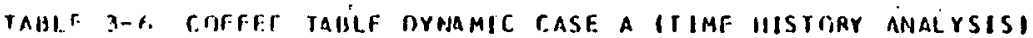

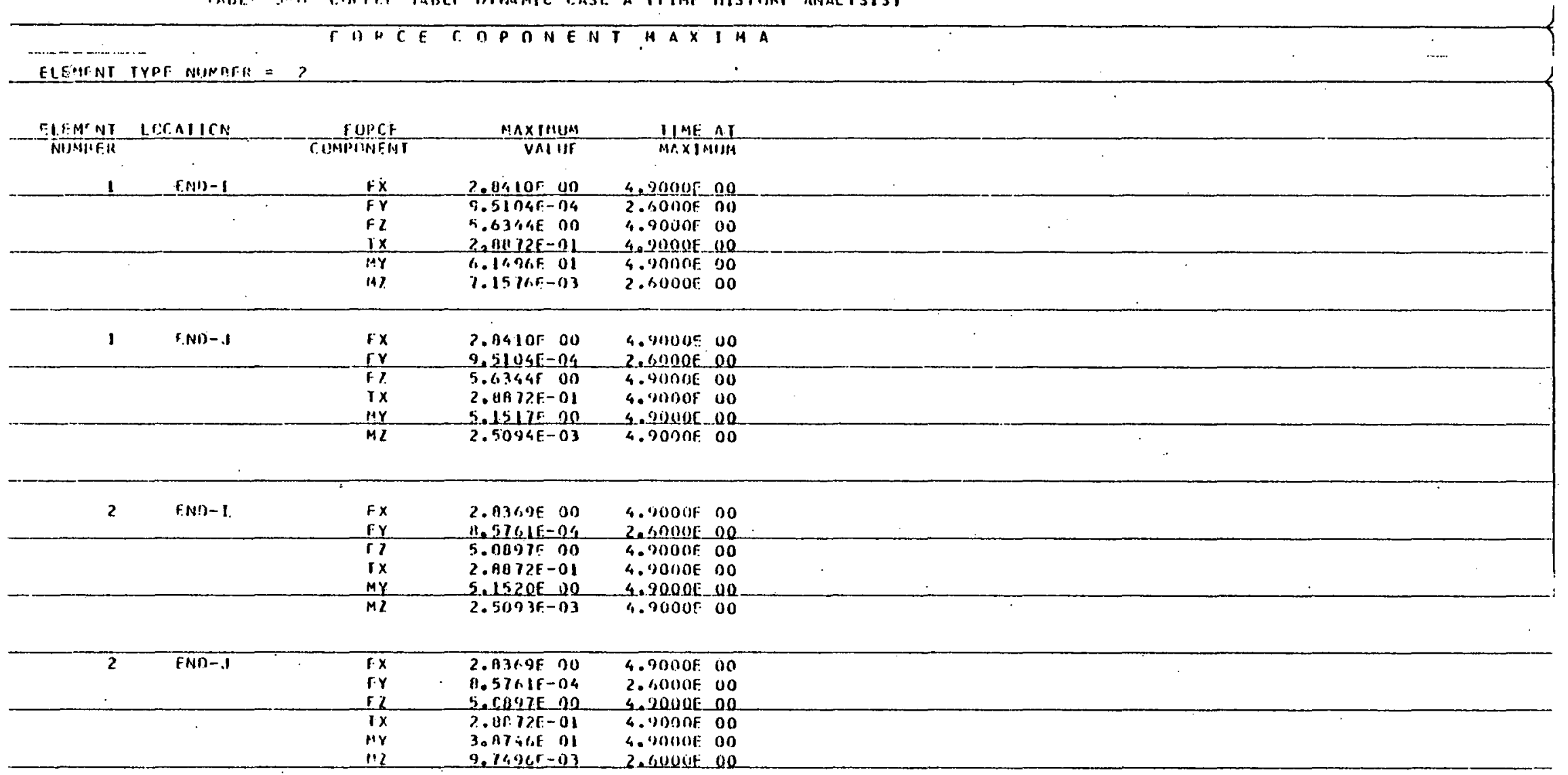

\begin{tabular}{|c|c|c|c|c|}
\hline 7 & SAPE-1 & $\begin{array}{l}F x \\
F y \\
1: z\end{array}$ & 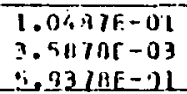 & $\begin{array}{ll}4.90010 \mathrm{r} & 00 \\
4.2000 \mathrm{O} & 00 \\
4.2000 \mathrm{e} & 00\end{array}$ \\
\hline & & $\begin{array}{l}\text { Ix } \\
\text { Mr } \\
\text { Ni }\end{array}$ & $\begin{array}{l}1.61: 74 \mathrm{E}-1) 3 \\
1.7 .410 \mathrm{O} \\
1.1301 \mathrm{E}-07\end{array}$ & $\begin{array}{l}4.9000600 \\
4.9000 F \text { 00 } \\
4.40010 E \text { Do }\end{array}$ \\
\hline 3 & FN(1)-J & $1 \cdot x$ & $1,04 \Omega 7 F-01$ & - sougr 01 \\
\hline & & $\begin{array}{l}F y \\
1: 7 \\
1 x\end{array}$ & $\begin{array}{l}3.5 n 79 F-0.3 \\
509378 F-01 \\
1.03745-03\end{array}$ & 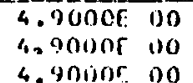 \\
\hline & & $\begin{array}{l}n y \\
117\end{array}$ & 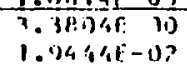 & 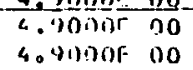 \\
\hline
\end{tabular}


$B \quad[(N)-1$

$\begin{array}{r}3 \quad(N)-1 \\ \hline\end{array}$

9

(N)

\begin{tabular}{r|r|}
$9 \quad$ (NO-J \\
\hline
\end{tabular}

9

\begin{tabular}{c}
$9 \quad$ (ND-1 \\
\hline \\
\hline
\end{tabular}

9

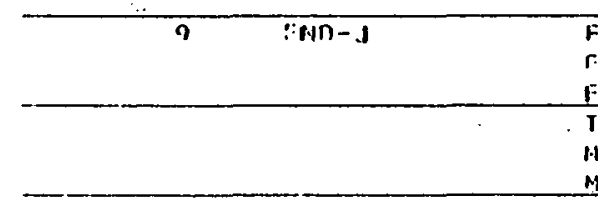

\begin{tabular}{|c|c|c|}
\hline $\begin{array}{l}F X \\
F Y\end{array}$ & $\begin{array}{l}1.04916-01 \\
3.02575-03\end{array}$ & $\begin{array}{l}4.9000 F \text { OO } \\
4.9000 E\end{array}$ \\
\hline $1=2$ & $5.93 \cap 3 E-0.1$ & $4.9000 \mathrm{~F}$ \\
\hline IX & $1 . \sin 045=03$ & 4.90005 \\
\hline$n y$ & 1.1415820 & .20000 \\
\hline
\end{tabular}

$\begin{array}{lll}1.1415 \mathrm{~F} 20 & 4.2000 \mathrm{E} 00\end{array}$

\begin{tabular}{|c|c|c|}
\hline $\begin{array}{l}F x \\
r y\end{array}$ & $\begin{array}{l}1.0491 E-01 \\
3.5251 E-03\end{array}$ & $\begin{array}{l}4.9000 \% \\
4.9000 F \\
400\end{array}$ \\
\hline Fl & $5.32 B 3[-01$ & $4.200 U E$ OO \\
\hline$T X$ & $1.66,04 \div-03$ & 4.9000500 \\
\hline HY & 3.3803500 & 4.9DODE DO \\
\hline$M l$ & $1.95 \mathrm{R2E}=02$ & $4.2900 \mathrm{E} 00$ \\
\hline
\end{tabular}

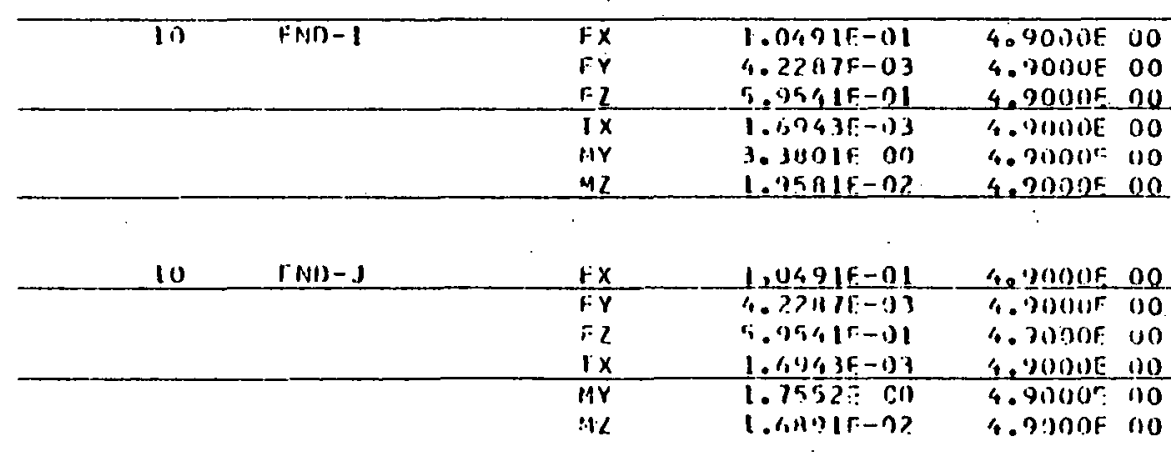

2.8370F CO 4.90OUF 00

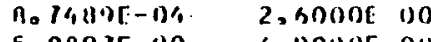

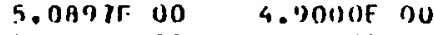

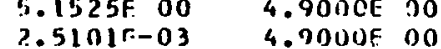

2.8370r no 4.9000F 00

A.74B9E-04 2.30OOF 00

5.01378 no 4.90006 00

2. BBSGE-OI 4.900OF 110

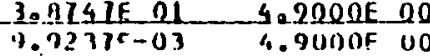

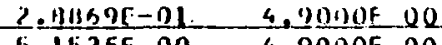

\begin{tabular}{|c|c|c|c|c|c|}
\hline 11 & (NN-1 & & $5 x$ & $3.13(, 05: 0)$ & $4.21300 \mathrm{r}$ \\
\hline & & & $\overline{i: \gamma}$ & $1.6170(-1) ?$ & $9.9600 \mathrm{n}$ \\
\hline & & & 17 & $5,11145 \div 010$ & 4. Domo: \\
\hline & $\therefore$ & $\therefore$ & $\cdot x$ & $\because=23+21-01$ & 4.9ongur \\
\hline
\end{tabular}




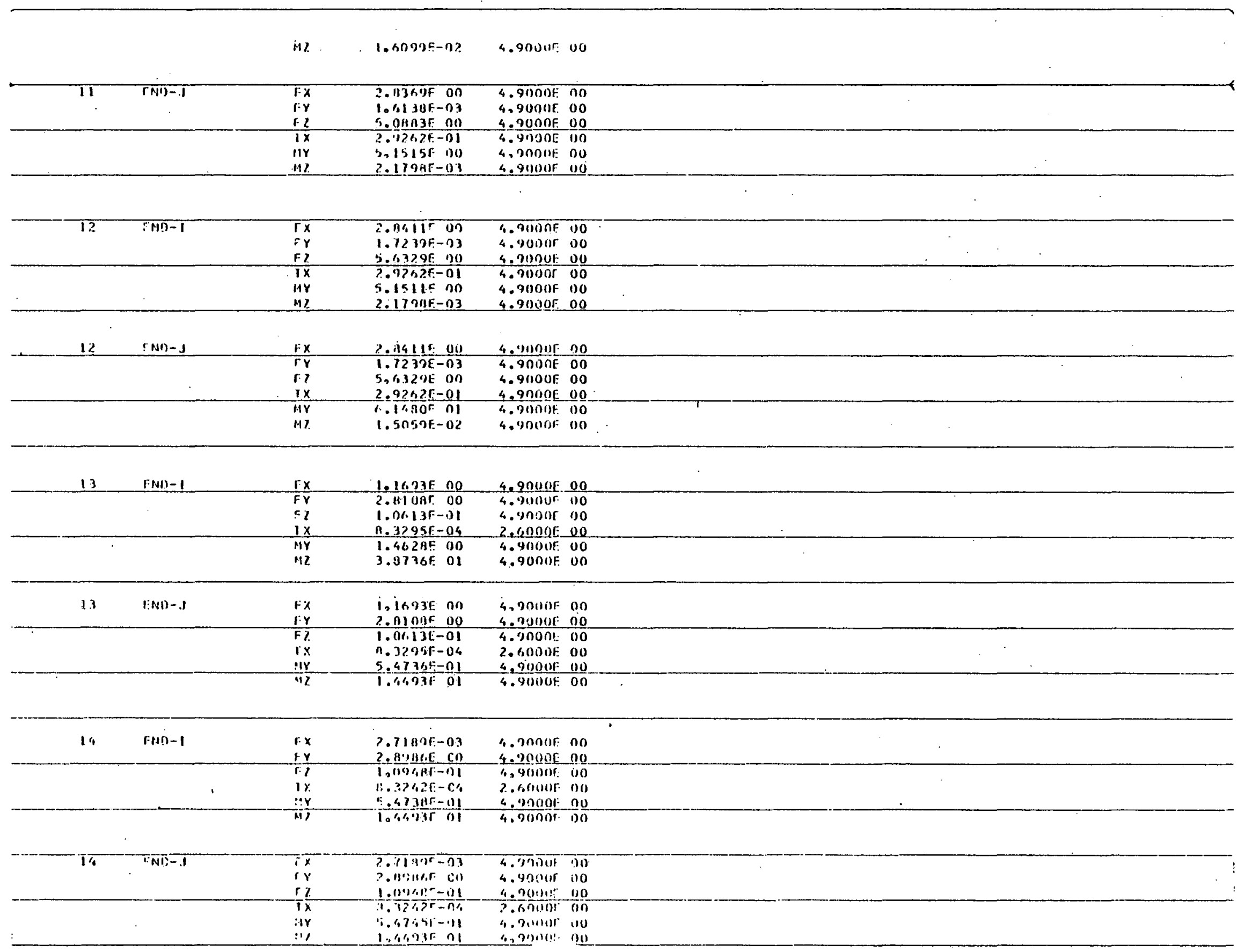




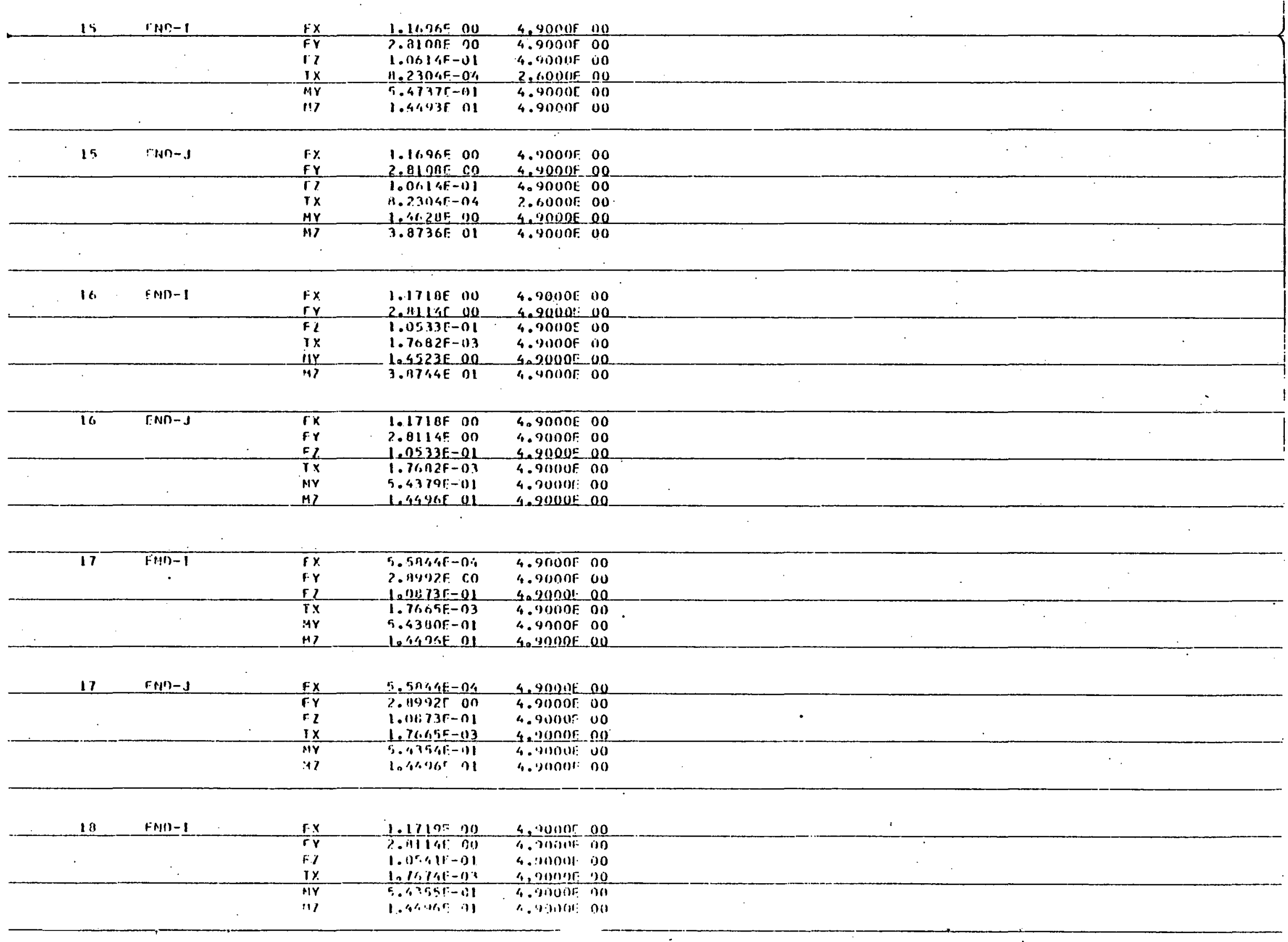




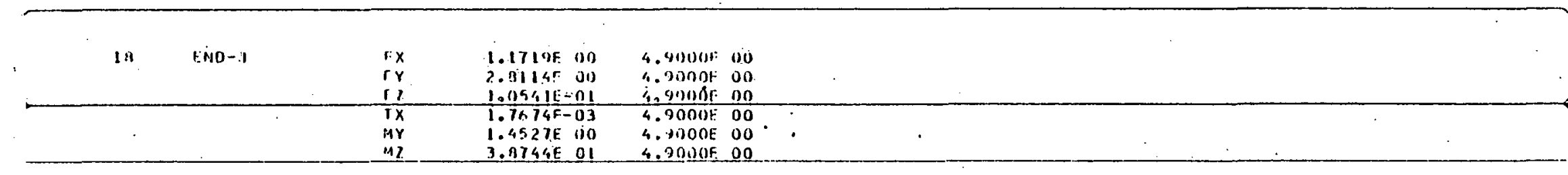


TAHI T-7 COMFFE TABLE UYHAHIC CASE A ITIME IISIOHY AHALYSISI

5 THFS.S C OMPONENT MAXIMA.

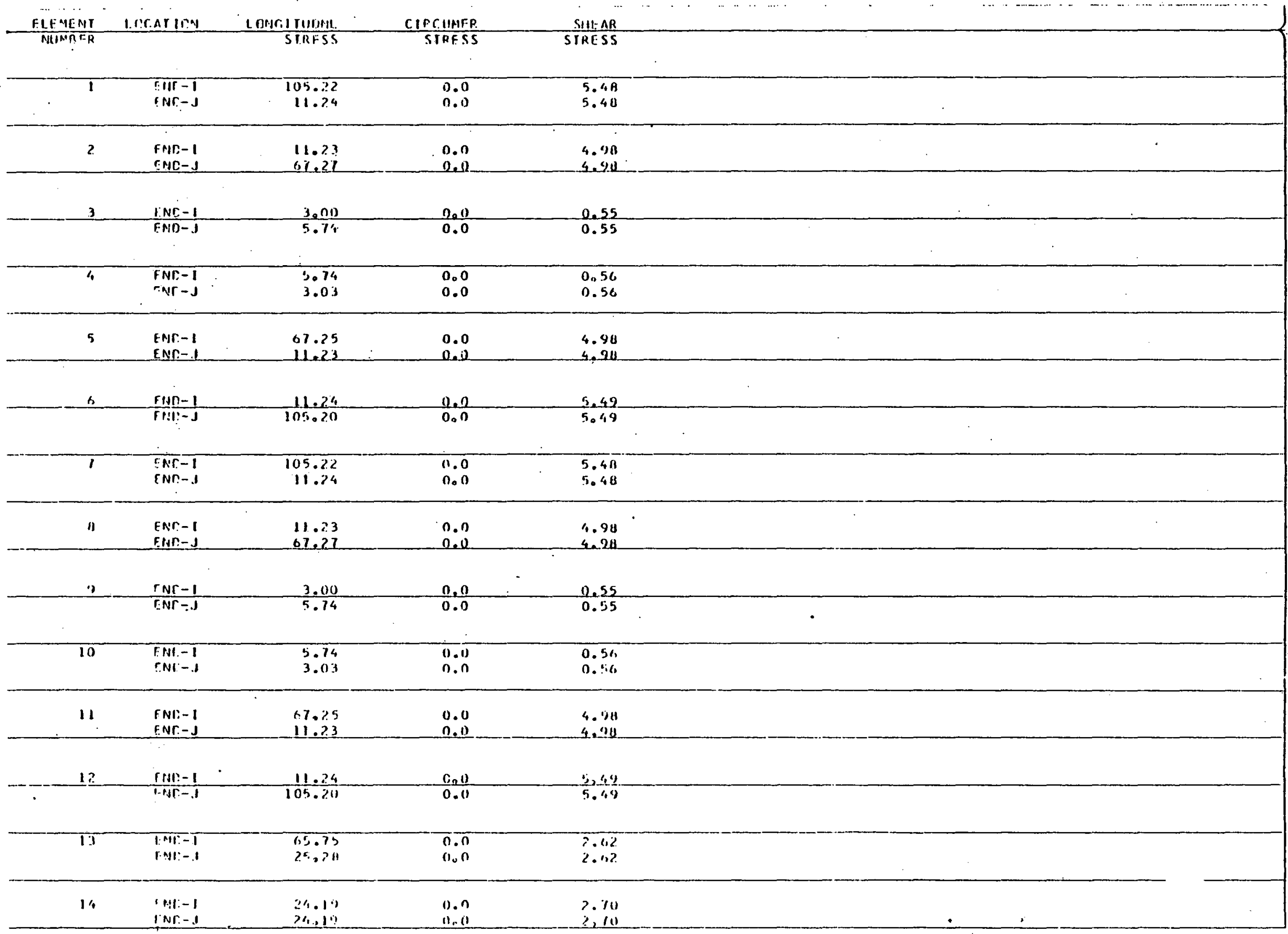




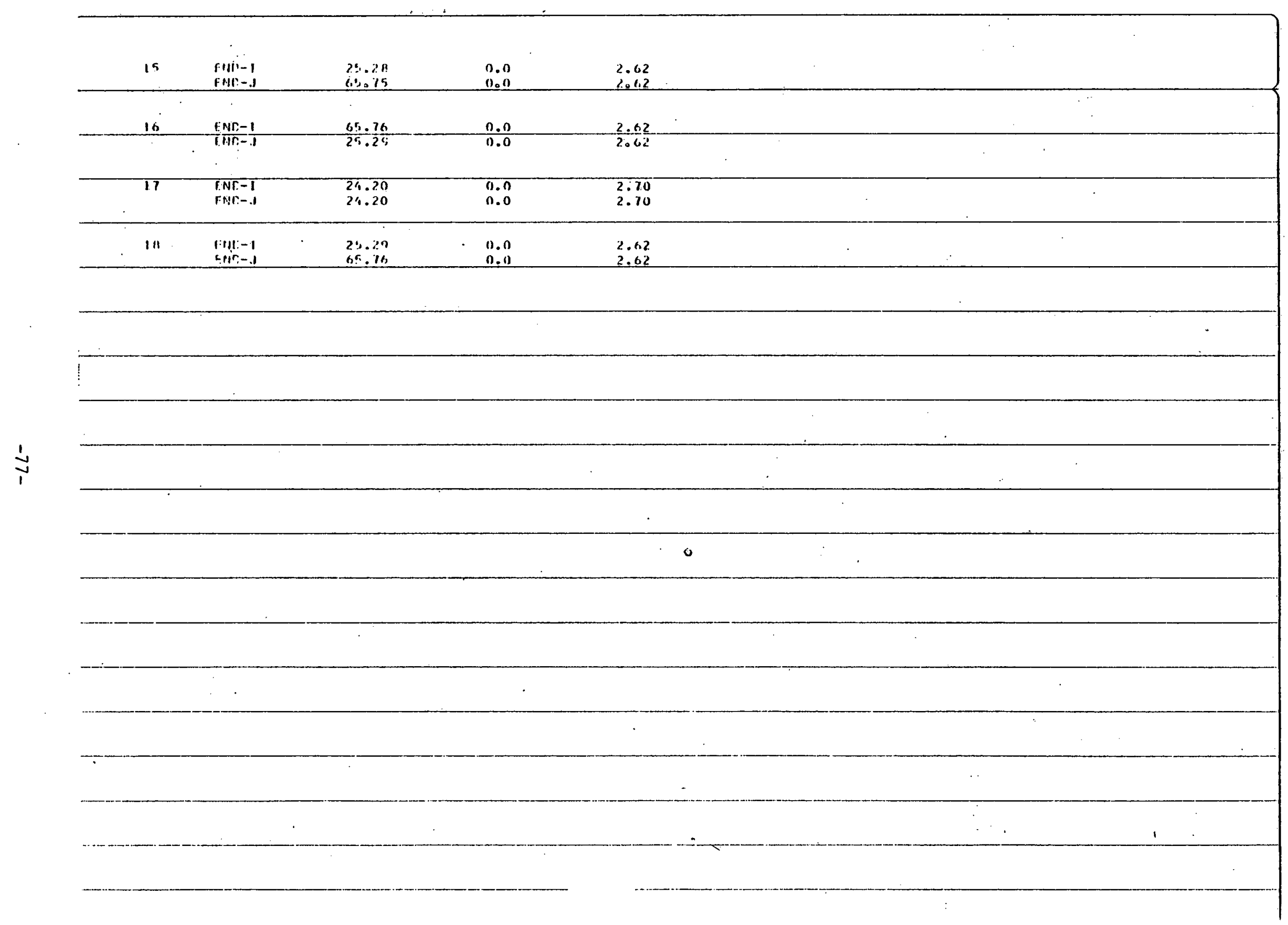




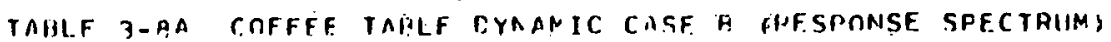

MGDOL FAHIICIPATIOA FACTORS AND EAFTHOUAKF. SPECTRUM

DIRECIION FACTOFS

$$
x=1.000 C \quad y=-0.0000 \quad T=-0.0000
$$

INDICATOR FCA DISPLACEMENT OF ACEFIEAATION SPECTRIIM =

EQ.0 DISPLACEMEAT

\begin{tabular}{|c|c|c|c|}
\hline MODAL & PAPTICIPATIUN & FACIOAS & . \\
\hline MODE & $X=$ DIFECTICN & YODIFFCTIOA & Z-DINECIION \\
\hline$i$ & $\because 4>53 F+00$ & $.5967 f-15$ & $.1297 E-07$ \\
\hline 2 & $.0129 F-19$ & $.50 .31 F-14$ & $.42 A O E+0 O$ \\
\hline 3 & $\because 1074 \mathrm{E}-11$ & $.4753 f-15$ & $.3487 E-\mathrm{I} 0$ \\
\hline 4 & $.4508 \mathrm{~F}-17$ & - $5645 F-1=$ & $.5>40 E-i .3$ \\
\hline 5 & $.1041 E-13$ & -.331 KE - $0 ?$ & $.73 .34 \mathrm{E}-17$ \\
\hline
\end{tabular}

SPECTAUM TAFLE IIMPEFIAL VALIEY F.O. FLCFATHO 1940 SE

NLMBEP CF PCINTS:E 11

SCALE FACTOF := $\quad$ IOOCOE+OI

\begin{tabular}{|c|c|c|}
\hline INPUT & & SPECTHLN \\
\hline POINT & PEFIOU & VAILLE \\
\hline 1 & .169AE-0? & $.1450 E-0.3$ \\
\hline$?$ & . 2 PKOE-OI & $: 390 \cap E-\cap 3$ \\
\hline 3 & SAROE-01 & .775 NE. O3 \\
\hline 4 & $.71 O O E-O I$ & $.775 \mathrm{nF} .03$ \\
\hline 5 & .910 OE-OI & .440 OE, OB \\
\hline$a$ & $.1140 \mathrm{~F}+00$ & DIAQE \# $\cap 4$ \\
\hline 7 & 161OF, ON & $.11 \cap \rho E \rightarrow 04$ \\
\hline B & .1720 Of $A n$ & $.700 \cap F+0.3$ \\
\hline 9 &. OONOE +ON & $.87 \mid C E+\cap 3$ \\
\hline 10 & $.750 \mathrm{NF}+00$ & $. A 7] C F \cap \cap 3$ \\
\hline 11 & $.323 n F+n n$ & $.4 \cap \cap \cap E+\cap ?$ \\
\hline
\end{tabular}


TAMLE 3-9a COFfFE TAPLE CYMANIC CASF B (DESPONSE SPECTRUM)

RFSPONSF SFECTRUM CISFLACEMENT CCNHTINENTS

RESPONSF FCE MOCE 1 H
FAEDUENCY

CISPIACFMENTS,AOTATIUNS DF UNGESTRAINEU NODFS

\begin{tabular}{|c|c|c|c|c|c|c|}
\hline $\begin{array}{l}\text { NONE } \\
\text { AUMHER }\end{array}$ & TRANSLATION- & THANSIATION & THANSLATION & POTATION & RCTATION & Fotation \\
\hline 1 & $-7 . \cap 9945 F-04$ & - 1.5P7AIE-OK & $3.33297 E-0 A$ & $-3.90 B 65 E-09$ & -3.407 & $3.00562 E-05$ \\
\hline 2 & $4449 \mathrm{E}-04$ & IE-OG & $E-\cap A$ & & & -05 \\
\hline 3 & $-2.08949 E-04$ & aIE-OK & $3 E-O B$ & -.3 & 3.407 & 3.00 \\
\hline 4 & -2.0 П94 SE - П४ & - I. SPTחIE-OK & $7 E-\cap A$ & $3.9 ! 048 E-09$ & $3.40757 E-07$ & $3 . n$ \\
\hline 5 & -4.490 & -2. & B & -2 & 6.3 .4 & -05 \\
\hline 6 & -4.56 & & & & 14 & -05 \\
\hline 7 & $-4.49095 E=04$ & -2.05530 & $-0 R$ & ?.0?0ZHE-09 & $-6.346 K 1 E-07$ & -05 \\
\hline ด & $-4.49660 E-1$ & 3.36 & & 7.416 & 1.943 & -06 \\
\hline 9 & -4.49 & $-3.3 f$ & -1. & -7. & $1.4436 \mathrm{RE}-07$ & -06 \\
\hline 10 & $-4.49 n 95 E-n 4$ & $\therefore .9553 \cap E-06$ & 5.0 & -2.01945 & -6.34 & -05 \\
\hline 1.1 & $-4 \cdot 5$ & & & -1 & $-1.49 E \cap A E-14$ & $.69 \mathrm{B9BE}-05$ \\
\hline 12 & $-4.49095 E-04$ & $2.4553 n F_{-}-76$ & $-5.05300 E-O A$ & $? .020286$ & $346 \times 1$ & \\
\hline 1. &. .4 & & & & -1. & \\
\hline 14 & $-4.496 \rightarrow 0 E-74$ & $3.36914 F_{-}-05$ & $-1.29980 E-06$ & -7.4 ก $17 E-10$ & $-1.443 .6 \mathrm{BE}-07$ & $T 1931 E-06$ \\
\hline
\end{tabular}

CISPLACEMENTS,FOTMIIONS OF UNHESTAAINED MODES

\begin{tabular}{|c|c|c|c|c|c|c|}
\hline $\begin{array}{l}\text { NODE } \\
\text { NUMAEA }\end{array}$ & IFANSLATION & THAASLATION. & TRANSLATIUUN & ROTATION & RCTATION & POTATIOÁ \\
\hline 1 & $3.77584 E-16$ & 2. $144.7 \cap E-15$ & 2. $A \cap 40 \cap F-13$ & $3.93976 E-14$ & $-1.20476 E-15$ & $7182 A E-17$ \\
\hline$r$ & $-3.7>\operatorname{SAgE}-16$ & 2. $.44 A 7 \cap F-15$ & .40400 & $3.93976 E-14$ & $20476 E-15$ & \\
\hline 3 & $.7 .72594 \mathrm{E}-16$ & $=2.94$ A TRE -15 & $\mathrm{HO}$ & 3.9797 KE-14 & 14 & -17 \\
\hline 4 & $-3.775 A 4 E-16$ & $-3.9497 \cap F-15$ & 2. $A \cap 4 \cap 0 E-13$ & $3.9 .3976 E-14$ & -1 & 17 \\
\hline 5 & - -4 . P5P PE - 16 & $-7.16296 E-15$ & $5.8 \cap 7 A 7 F-13$ & 1. AOn32E-14 & 4 ?A7E-15 & -3.67 \\
\hline 6 & A. . 1 ก97E - ?? & E. F. A OAR - 24 & -13 & $-4 . A \cap 230 E-15$ & 614 & -3. \\
\hline 7 & $6.4 P 59 n E-16$ & $7.1629 \mathrm{At}-15$ & -1.3 & 1.. BOO3 $2 \mathrm{E}$ & 15 & \\
\hline 8 & & F. -15 & 13 & $E-14$ & -2.965 & 17 \\
\hline 9 & $-2.3 A 13 E E-16$ & $7.450+4 E-15$ & $6.1599 ? F-13$ & $1 . H \cap 032 E-14$ & 15 & \\
\hline 10 & $-6.4 A 5$ & 7.16 & & $1 . A \cap C$ & 15 & -18 \\
\hline 11 & P. $(1) \cap 41 E-? ?$ & $-5.6437 \mathrm{HE}-$ & 5.9 & $-4 . A \cap 230 E-15$ & $-6.36143 E-16$ & -3.20688 \\
\hline 17 & 6. 4 ASESE-16 & $-7.1+296 \mathrm{At}-15$ & 5. & $1 . A_{0}$ & & \\
\hline 13 & $3.3 P_{13}$ & $-7.45044 E-15$ & $? F-1.7$ & $1.80032 E-14$ & $2.5+5+6 E-15$ & $2.72594 E-17$ \\
\hline 14 & $-7.34133 E-16$ & $-7.45044 t-15-$ & $6.15997 E-13$ & $1 . A \cap n 32 F-14$ & P.56GE-15 & -2.72593 \\
\hline
\end{tabular}


TAHLF 3-9P COFFEF TAPLE CYMAMIC CASF a (DESPONSE SPECTRUN)

RF SHOASF SFECTHUM CISFLACEMENT CCNPANENTS

FESPONSE FCA NCC.E 3
FREOUEACY $=137.161^{3}+7$

CISPLACFMENTS, FOTATIUNS OF UNHESTRAINFO NOCES

\begin{tabular}{|c|c|c|c|c|}
\hline $\begin{array}{l}\text { NOIJE } \\
\text { AUMAER }\end{array}$ & TEANSLATION & $\begin{array}{r}Y- \\
\text { THAASLATION }\end{array}$ & THÁINSLATION & nOtATIOA \\
\hline 1 & $-1.91415 E-1 \mathrm{~A}$ & $2.7 \cap 035 E-18$ & $3.203625-16$ & $4.4 ? 525 F-17$ \\
\hline 2 & $-1.91415 E-16$ & $-\bar{Z}-7 \cap 035 E-I A$ & $-3.2 n 3625-16$ & $-4.42525 E-17$ \\
\hline 3 & $1.91415 E-16$ & 2.7nO35E-1B & $-3.2036 ? E-16$ & $\begin{array}{r}-4.42525 E-17 \\
4.42525 E-17\end{array}$ \\
\hline 4 & $1.91415 E-16$ & $-\bar{E} .1 C 035 E-18$ & $62 F-16$ & $\begin{array}{l}4.47525 E-17 \\
1.77333 E-17\end{array}$ \\
\hline 5 & $\begin{array}{r}\text { 3.92779E-16 } \\
-1.7735 \text { AE -27 }\end{array}$ & $\begin{array}{l}-5.02424 E-18 \\
-1.47456 E-79\end{array}$ & $\begin{array}{l}6.48054 E-16 \\
6.4 A G 74 E-16\end{array}$ & $-5.04306 E-18$ \\
\hline 7 & $-3.9 ? 779 E-16$ & $5.024 ? 4 E-1 A$ & 6.4A054F-J6 & 1.77333 \\
\hline A & $3531 E-16$ & $=.73 n E>P F-17$ & $2.51210 F-16$ & $6.50763 E-18$ \\
\hline 9 & $-3.93531 E-16$ & $-\hat{\varepsilon} .7 ? 06 ? E-17$ & $-2.51210 E-16$ & -6.50763 \\
\hline 10 & 2775 & $-5.02424 F-1 n$ & $54 F-16$ & -1.7733 \\
\hline 11 & $-1.00951 E-27$ & $7.46775 t-30$ & $-6.4 A 674 F-16$ & 5.04306 \\
\hline 12 & 3 & $5 . n 2424 \mathrm{E}-1 \mathrm{~A}$ & $-6.4 A \cap 54 E-16$ & 77333 \\
\hline 13 & $3.9353, E-16$ & $2.73062 E-17$ & $-2.51210 E-16$ & $-6.5 n 7 \in 3$ \\
\hline 14 & $3.93531 E-16$ & $-2.7 .206>P E-17$ & $.51 .210 E-16$ & $6.5 n 763 E-18$ \\
\hline
\end{tabular}

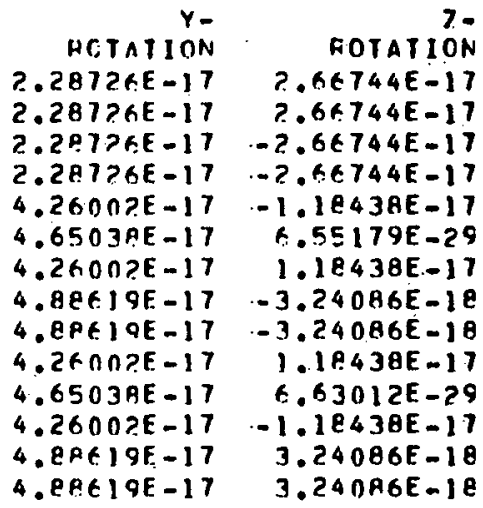

FESPONSE FCR MCCE

FREDUENCY $=215.797$

$+$

CISPLACEMENTS,FOTATIONS OF UNRESTRAINFD AOCES

NODE.
AUMAER
1
2
3
4
5
6
7
9
9
10
11
12
13
14

$X-$
TEANSLAIION
$4.4117 S E-17$
$4.61179 E-17$
$-4.61175 E-17$
$-4.61179 E-17$
$-9.19704 E-17$
$3.26624 E-2 A$
$9.19704 E-17$
$9.24773 E-17$
$9.24073 E-17$
$9.19704 E-17$
$-9.1926 E E-? 9$
$-9.19704 E-17$
$-9.24073 E-17$
$-9.74072 E-17$

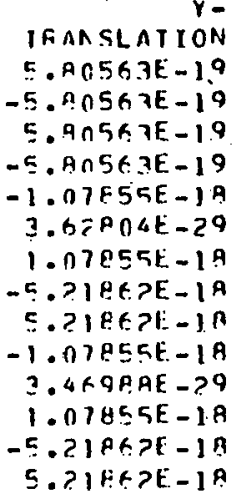

$Z-$
THANSLATIUN
$3.00727 E-17$
$-3.00727 E-17$
$-3.00727 E-17$
$3.00727 F-17$
$5.91067 F-17$
$5.97467 F-17$
$5.91067 E-17$
$4.091695-17$
$-4.091695-17$
$-5.91067 F-17$
$-5.97467 E-17$
$-5.91067 E-17$
$-4.09169 F-17$
$4.09164 F-17$

ROTATIOA

HOTATION $-2.35511 E-1 A$ 4. O? $-4 . \cap 2 A P 3 E-18$ $-4 . \cap 2 A A 3 E-18$ $4.02 A B 3 F-18$ $1.59584 E-18$ $-3.54101 E-19$ 1. 5 A $584 E-18$ 5. $1960 E-19$ $-5.81960 E-19$ $-1.5 A 5 A 4 E-18$ $3.54101 E-19$ 3.54 तIE- 19 5. 1960 196-19 $-2.35511 E-1 A$ $-2.35511 E-1 B$ - 4 U - I. Z7? PIE-17 4. JPE JPE-1A $6.4345 \mathrm{nE}-1 \mathrm{~A}$ $5.43450 E-1 B$ -4 . उ В 3 AF -1 A $-4.38+3 R E-1 A$ $6.4345 \cap E-1 \mathrm{~A}$ $6.4345 \cap E-1 A$ 
IAHLF 3-GC COFFEF TAPLF CYMANTC RACF G IRESPONSE SPECTRUM,

HFSPONSF SFECTRLIM RISFLATEMERT CCNPOAENTS

PESPONSE FCA NOCE 5
FREOUFACY $=404.275^{2}+7$

PISPLACENENTS, FOTATIONS OF UNAESTHAINEN MONFS

\begin{tabular}{|c|c|c|c|}
\hline $\begin{array}{l}\text { ONF. } \\
\text { A AE } R\end{array}$ & $\begin{array}{r}x= \\
\text { IFANSLAIION! }\end{array}$ & JIAASLATION & TRANSLATION \\
\hline 1 & $-2.23 \cap 4 \cap E-2 ?$ & $-4.37079 E-22$ & $-1.145 ? 7 F-201$ \\
\hline 2 & $2.53 \cap 40 E-27$ & $-4.37079 F_{-}-2 ?$ & $-1.14527 F-20$ \\
\hline 3 & ?.2304OE-?? & $-4.370796-7 ?$ & $1.14527 F-20$ \\
\hline 4 & $-2.23040 E-7 ?$ & $-4.370795-7 ?$ & $1.14527 f-20$ \\
\hline 5 & $0.29472 f-21$ & $-P .0 \% 80 K E-2 ?$ & $2.13986 E-20$ \\
\hline$\ddot{n}$ & $5.732 \geqslant 7 E-19$ & 3. . F $789 E_{-}-21$ & $9 \mid F-31$ \\
\hline 7 & A.?9672E-21 & -P.NEBORE -22 & -2.1 .798 KE - 20 \\
\hline A & 3.OK? 5 SE -DI & $-z .16>2>F-20$ & $-1 . \geqslant 296, F-19$ \\
\hline 9 & $-3.06 ? 59 E-? 1$ & $-2 . i+222 E-20$ & $796, F-18$ \\
\hline 10 & $-H .2947 ? E-2 !$ & 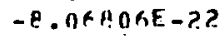 & $-2.13986 F .-20$ \\
\hline in & $-5.7 .72727 E_{-19}$ & $3 .>4.72$ AE -21 & -3.0365 AF -.31 \\
\hline i? & $-8.29472 E-21$ & 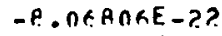 & $2.13986 E-? 0$ \\
\hline 13 & $-3.06259 E-21$ & $-2.1+2.2>E-20$ & $1.22996 \mid E-19$ \\
\hline 14 & $3.00 ? 59 E-21$ & $-5.1 \times 2 ? 2 t-20$ & $1.2796, E-1 H$ \\
\hline
\end{tabular}

\begin{tabular}{|c|c|c|}
\hline Rotarion & RCIATION & ROTATIOA- \\
\hline $\mid 312 F-21$ & B. $1340 ? E-20$ & $-3.30229 E-22$ \\
\hline$-1.4 \mathrm{~B} 312 \mathrm{E}-21$ & $-6.134 n 25-20$ & $3.36229 E-22$ \\
\hline $1.4 n 312 F-21$ & $6.134 n>E-? .0$ & .72 \\
\hline 0.31 & $\begin{array}{l}12 E-20 \\
A E-19\end{array}$ & $\begin{array}{l}-22 \\
-21\end{array}$ \\
\hline-3 & InOE - 32 & $-1.93594 \mathrm{E}-21$ \\
\hline$-A .49 ? 29 E-22$ & $1.14246 E-19$ & $93594 E-71$ \\
\hline$-8.49 ? 2$ & 9.4047 AE -20 & $-1.62699 E-21$ \\
\hline-8.492 & 7 PE -20 & 1.62 \\
\hline$-A .49229 F-22$ & $-1.14 \approx 46 E-19$ & $1.93594 E-21$ \\
\hline 1. KOAA IE-33 & -8.0390 P.F -33 & $1.93594 E-21$ \\
\hline $9 E-2.2$ & OATAE - ? 0 & $62699 E-21$ \\
\hline B. $49 ? 29 E-? Z$ & -9.4047 PF. -20 & $-1.62699 E-?$ \\
\hline
\end{tabular}

SOUARE ROUT OF HTF. SUM GF THF SOUARES OF THE HODAL DEFLECIIONS

CISFLACENFATSARTTATIONS OF UNHFSTRATMEO AODES

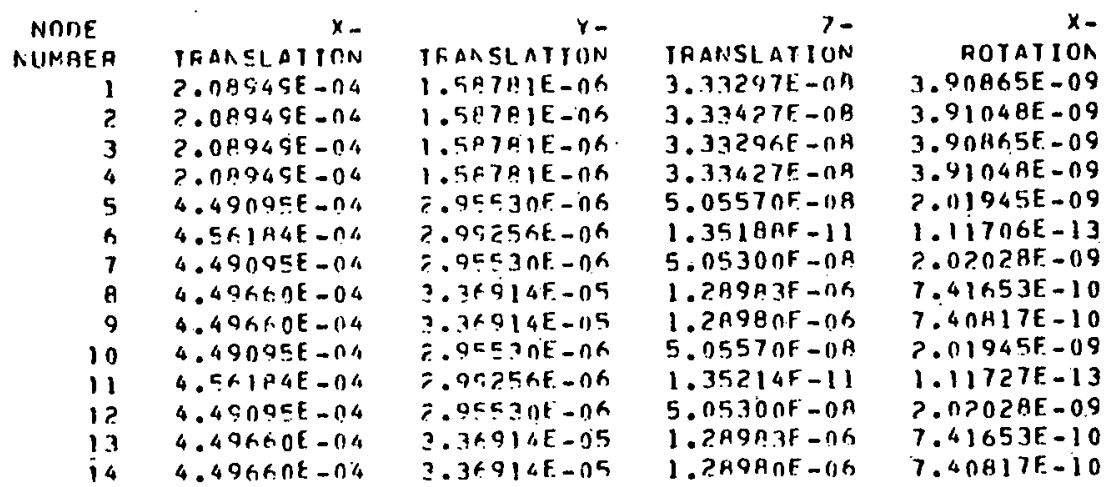

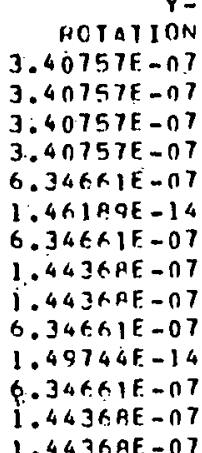

ROTATIOA $3.00562 E-05$ $3.00562 E-05$ $3.10562 E-05$ $3.00562 E-05$

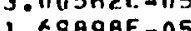
$1.09898 \mathrm{~F}-05$ $1.69898 F_{.}-05$ $1.69 B 9 B E-05$ $3.71931 E-06$ $3.71931 E-06$ 1. GSA9AE-05 1.69A9HE - 05 $1.6989 A E-05$ $3.71931 E-n 6$ 3.71931 F. 06 
TABLF 3-10 CRFFEE TAPLE CYAAMTC CACF. A (AESPONSE-SPECTRUM)

RF SPONSE SFECIPUM FCRCE CUMPONENIS

SCUAHE BOOT OF ItF SUN OF THF SGUARES OF THE NODAL. FORCES

(FOH ALL. ELFNENIS)

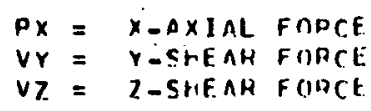

IX $=$ X-TWISTING MONFIAT

$V Z=$ Z-SHEAH FOBCE

MY $=Y$ - PENCINE MONEA

$M Z=2-$ AENCING MONEAT

$I=$ INITIAL NONE

$C=$ CENTER NOCF (FOH PIFE HEAD ONLY)

$J=$ FINAL NONE

ELEMEAT TYPF $13 / C$ P IDE

1,1 ELENENT NIIMGER $(11$

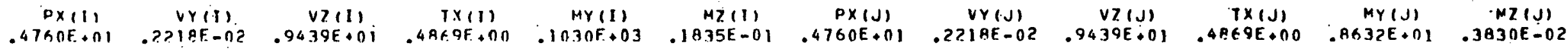

ELEMENT TYPF $13 /$ C F I P F

1,1, ELEMENT NIJMPEA 121

DXII) VYII VIII

IXII) MYII) MZ(I) $P X(⿻)$

$.4753 E+01.2073 E-02.8527 F+01$ $4 B 69 F .00 \quad$ A63

$.4753 \mathrm{E}+01$

VY (J)
$2073 E-02$

VI. (J)

$1 \times(J)$
40696.00

MY (J)

$M z(J)$

L: ELEMEN TYPF $13 / C$ P I P F

1,1, ELEMENT NIJMPEP , 3)

PXII) VY(I) VZII)

IXII) MY(I) $M z(I) \cdot P \times(J)$

0.07
$V Y(J)$

V. (J)

(1)

$.6491 E+02 \quad .2171 E-01$

$.1757 E+n \cap$. BS?ZE-RE .996OF+RO

$7149 F-10$.29?RE+01 .23A2E-01 .1757E+DO .6533E-02

$9960 E+00 \quad .7149 E-10$

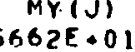

$M(2)(J)$

$.3253 E-01$

ELEMFA TYHE $13 / 0$ F I P F.

1,1, FLENEAT NIIMBER 141

$P \times(l)$

vyil

VIII)

$(x)(1)$

$M Y(1) \cdots M Z(1)$

$\operatorname{Px}(2)$

$V Y(J)$

V) ( $)$

$M Y(J)$
$M P E+01$

M2(J)

ELEREAT TYPF $13 / \mathrm{B}$ P I P E

$1 \because 1$, ELEMENT MUIMEER 151

$P \times(1)$
$.4753 E+01$

VY(I)

V. (1)

(X) (1)

MY (I)

MI (T)

PX(J)

527E+ OI

.4 ee $9 E+100$

MY (J)

$.8632 E+01$

$M 2(J)$ $.4969 F+0) .6491 F+02 \quad .2171 E-01 \quad .4753 \mathrm{E}+01$.207.3E-n?

.

ELEMEAT TYPF 13,0 F I PE

1,1, FLENEAT NIIMHEF 161

$P \times(1)$
$.4760 E+n$

VYII)

vili

$(x \times(1)$

$\operatorname{liY}(1)$

$M>(1)$

$P \times(J)$

VY(J)

$.0439 F+01$

4AG DE. AO

Bค. $32 F_{-}+01$

. ?A TOE-0?

$.4760 E+01$

$.2219 E-n$ ?

$.97(J)$
$.9430 E+01$

$T \times(J)$
$4 e E 9 E+100$

$M r(J)$
$1030 E+0.3$$\quad$ if -01 


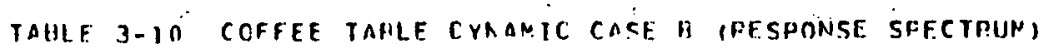

AFSPONSE SFECTRUM FCACE COMPONENTS

Element TYPF 130 P I "

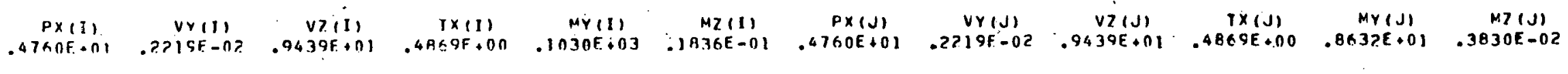

Element type 13,0 f

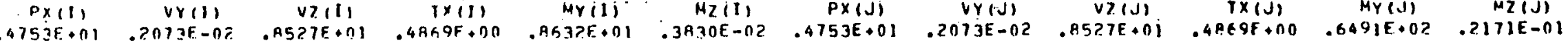

ELEMENT TYPF (3/R P I P F , , , ELEMENT NIJMAFA 19$)$

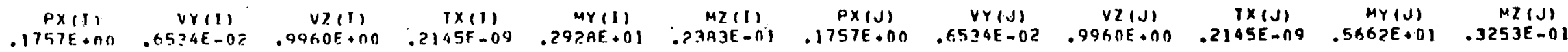

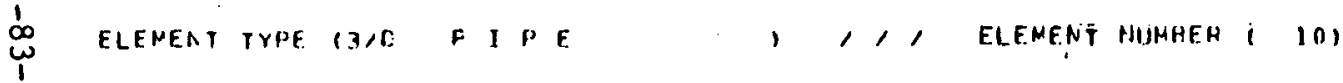

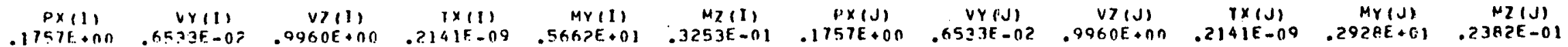

Element trPe 13/6 P I PE

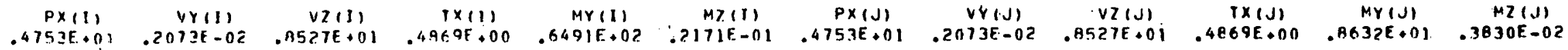

elemeat trPe 13, P I P F

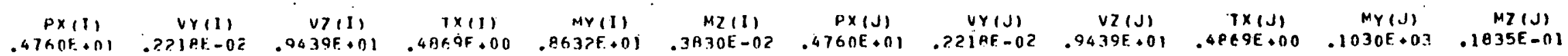

ELEMEN T TYPE $13 / 0$ P I P F $, 1,1$ FLENEAT NIMAER 1 (3)

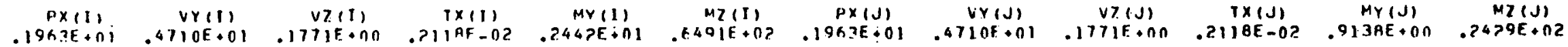

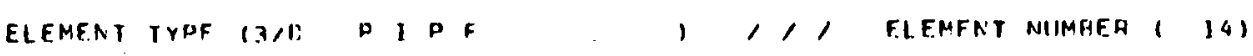

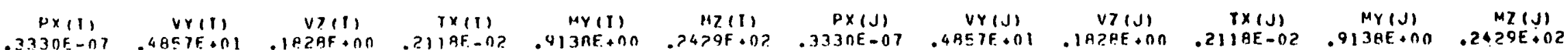


TAHLE 3-10 COFFEF TABLF CYMANTC CASE H (RESOONSE SFECTRUM)

HFSPOASE SFECTPUM F.CACE CONFONENIS

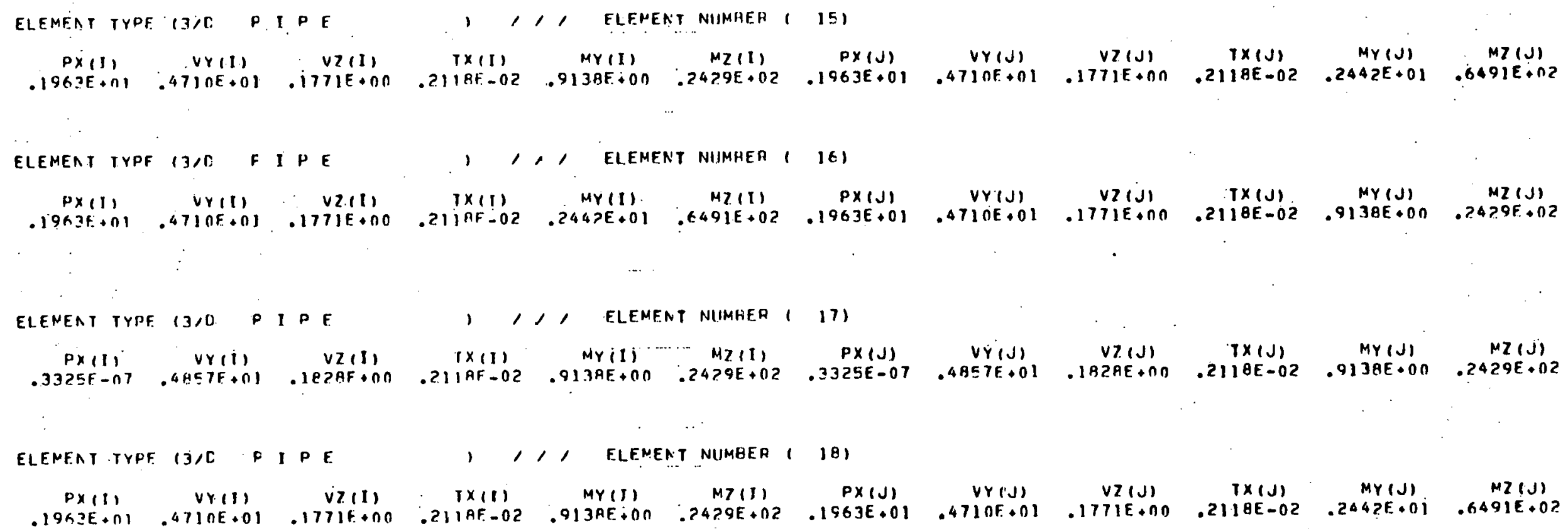




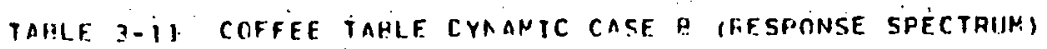
RF SPONSE SPECTRUM STRESS CONPONFNIS

\begin{tabular}{|c|c|c|c|c|c|c|}
\hline $\begin{array}{l}\text { ELFMENT } \\
\text { NUMAEA }\end{array}$ & $\begin{array}{r}\text { ELENENT } \\
\text { TYPE }\end{array}$ & $\begin{array}{l}\text { LCAO } \\
\text { CASE }\end{array}$ & STATIOA & $\begin{array}{l}\text { IH:GTOL } \\
\text { STRESS }\end{array}$ & $\begin{array}{l}\text { CIACI:MF } \\
\text { STHESS }\end{array}$ & $\begin{array}{r}\text { SHEAA } \\
\text { SIHESS }\end{array}$ \\
\hline 1 & TAAGENT & 1 & $\begin{array}{l}E \cap C-1 \\
E \cap C-D\end{array}$ & $\begin{array}{r}19.19 ? \\
19.8 ? 7\end{array}$ & $\begin{array}{l}0.0 n n \\
0.0 n 0\end{array}$ & $\begin{array}{l}9.219 \\
9.219\end{array}$ \\
\hline 2 & TAAGENT & $\mathrm{i}$ & $\begin{array}{l}\text { EAC-1 } \\
\text { EAC:- }\end{array}$ & $\begin{array}{r}19.921 \\
120.205\end{array}$ & $\begin{array}{l}0.000 \\
0.000\end{array}$ & $\begin{array}{l}8.369 \\
8.369\end{array}$ \\
\hline 3 & TAMGFNT & 1 & $\begin{array}{l}\text { EA }[-1 \\
\text { EA }:-J\end{array}$ & $\begin{array}{r}5.387 \\
1.0 .263\end{array}$ & $\begin{array}{l}0.0 n n \\
0.0 n 0\end{array}$ & $\begin{array}{l}.927 \\
.927\end{array}$ \\
\hline 4 & TAAGENT & 1 & $\begin{array}{l}E \cap[-I \\
\operatorname{En}[-J\end{array}$ & $\begin{array}{r}1.0 .263 \\
5.387\end{array}$ & $\begin{array}{l}0.0 n n \\
0.0 \cap 0\end{array}$ & $\begin{array}{l}.927 \\
.927\end{array}$ \\
\hline 5 & TANGENT & 1 & $\begin{array}{l}E \wedge[-I \\
E \wedge C-J\end{array}$ & $\begin{array}{r}120.205 \\
1: 9.821\end{array}$ & $\begin{array}{l}0.0 \cap 0 \\
0.000\end{array}$ & $\begin{array}{l}8.369 \\
8.369\end{array}$ \\
\hline 6 & TAAGENT & 1 & $\begin{array}{l}E \cap C-1 \\
\operatorname{EnC}-J\end{array}$ & $\begin{array}{r}19.827 \\
198.197\end{array}$ & $\begin{array}{l}0.0 n n \\
0.0 n 0\end{array}$ & $\begin{array}{l}9.219 \\
9.219\end{array}$ \\
\hline 7 & TAMGEHI & 1 & $\begin{array}{l}E \wedge C-1 \\
F \wedge C 2]\end{array}$ & $\begin{array}{r}198.192 \\
1.9 .827\end{array}$ & $\begin{array}{l}0.000 \\
0.000\end{array}$ & $\begin{array}{l}9.219 \\
9.219\end{array}$ \\
\hline $\boldsymbol{A}$ & TAAGENT & 1 & $\begin{array}{l}E \cap C=I \\
\text { EAC }=J\end{array}$ & $\begin{array}{r}19.821 \\
120.205\end{array}$ & $\begin{array}{l}0.000 \\
0.0 \cap 0\end{array}$ & $\begin{array}{l}8.369 \\
8.369\end{array}$ \\
\hline 9 & TANGENT & 1 & $\begin{array}{l}\text { FAC I I } \\
\text { FAC }- \text { J }\end{array}$ & $\begin{array}{r}5.387 \\
10.263\end{array}$ & $\begin{array}{l}0.0 \cap 0 \\
0.0 n 0\end{array}$ & $\begin{array}{l}.927 \\
.927\end{array}$ \\
\hline 10 & TANGENT & 1 & $\begin{array}{l}E \cap[-1 \\
E \cap C-J\end{array}$ & $\begin{array}{r}10.263 \\
5.387\end{array}$ & $\begin{array}{l}0.0 n n \\
0.0 n n\end{array}$ & $\begin{array}{l}.927 \\
.927\end{array}$ \\
\hline 11 & TANGENT & 1 & $\begin{array}{l}\text { EAL-I } \\
\text { EAC-J }\end{array}$ & $\begin{array}{r}1 \geqslant 0.205 \\
19.8 ? 1\end{array}$ & $\begin{array}{l}0.000 \\
0.000\end{array}$ & $\begin{array}{l}8.369 \\
8.369\end{array}$ \\
\hline 12 & TANGEHT & 1 & $\begin{array}{l}E \wedge C-I \\
E \wedge C-J\end{array}$ & $\begin{array}{r}14.827 \\
188.19 ?\end{array}$ & $\begin{array}{l}0 . \operatorname{lnn} \\
0 . \operatorname{nno} 0\end{array}$ & $\begin{array}{l}9.219 \\
9.219\end{array}$ \\
\hline 13 & TAAGENT & 1 & $\begin{array}{l}E \wedge C-I \\
E \wedge C-J\end{array}$ & $\begin{array}{r}117.690 \\
45.177\end{array}$ & $\begin{array}{l}0.000 \\
0.0 n \pi n\end{array}$ & $\begin{array}{l}4.388 \\
4.3818\end{array}$ \\
\hline 14 & IAAGENI & 1 & $\begin{array}{l}\text { EAC:- } \\
\text { FAR:-J }\end{array}$ & $\begin{array}{r}43.350 \\
43.350\end{array}$ & $\begin{array}{l}n .0 n 0 \\
0.0 n n\end{array}$ & $\begin{array}{l}4.525 \\
4.52 .5\end{array}$ \\
\hline 15 & TAAGENT & 1 & $\begin{array}{l}\text { EAL-I } \\
\text { EAC-J }\end{array}$ & $\begin{array}{r}45.177 \\
117.690\end{array}$ & $\begin{array}{l}0.0 \cap 0 \\
0.0 \cap n\end{array}$ & $\begin{array}{l}4.388 \\
4.388\end{array}$ \\
\hline 16 & TAAGENT & 1 & 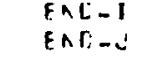 & $\begin{array}{r}17.650 \\
45.177\end{array}$ & $\begin{array}{l}0.0 n n \\
0.0 n n\end{array}$ & $\begin{array}{l}4.388 \\
4.38 B\end{array}$ \\
\hline 17 & TANGENS & 1 & $\begin{array}{l}\text { EAr. }-1 \\
\text { Etic }-J\end{array}$ & $\begin{array}{r}4.3 .350 \\
4.3 .350\end{array}$ & $\begin{array}{l}n .0 n n \\
0.0 n n\end{array}$ & $\begin{array}{l}4.525 \\
4.525\end{array}$ \\
\hline IA & TAAGENY & 1 & $\begin{array}{l}E \wedge C-1 \\
F \cap i-J\end{array}$ & $\begin{array}{r}45.177 \\
117.501\end{array}$ & $\begin{array}{l}n .0 n n \\
n .0 n n\end{array}$ & $\begin{array}{l}4.3 B A \\
6.3 H A\end{array}$ \\
\hline
\end{tabular}


TAPI + 3-12: C, GFFFF TABLF DYNAMIC CASE C ISTEP-BY-SIEP INTEGRATIONI

DISPI.AC. F M F N MAXIMA

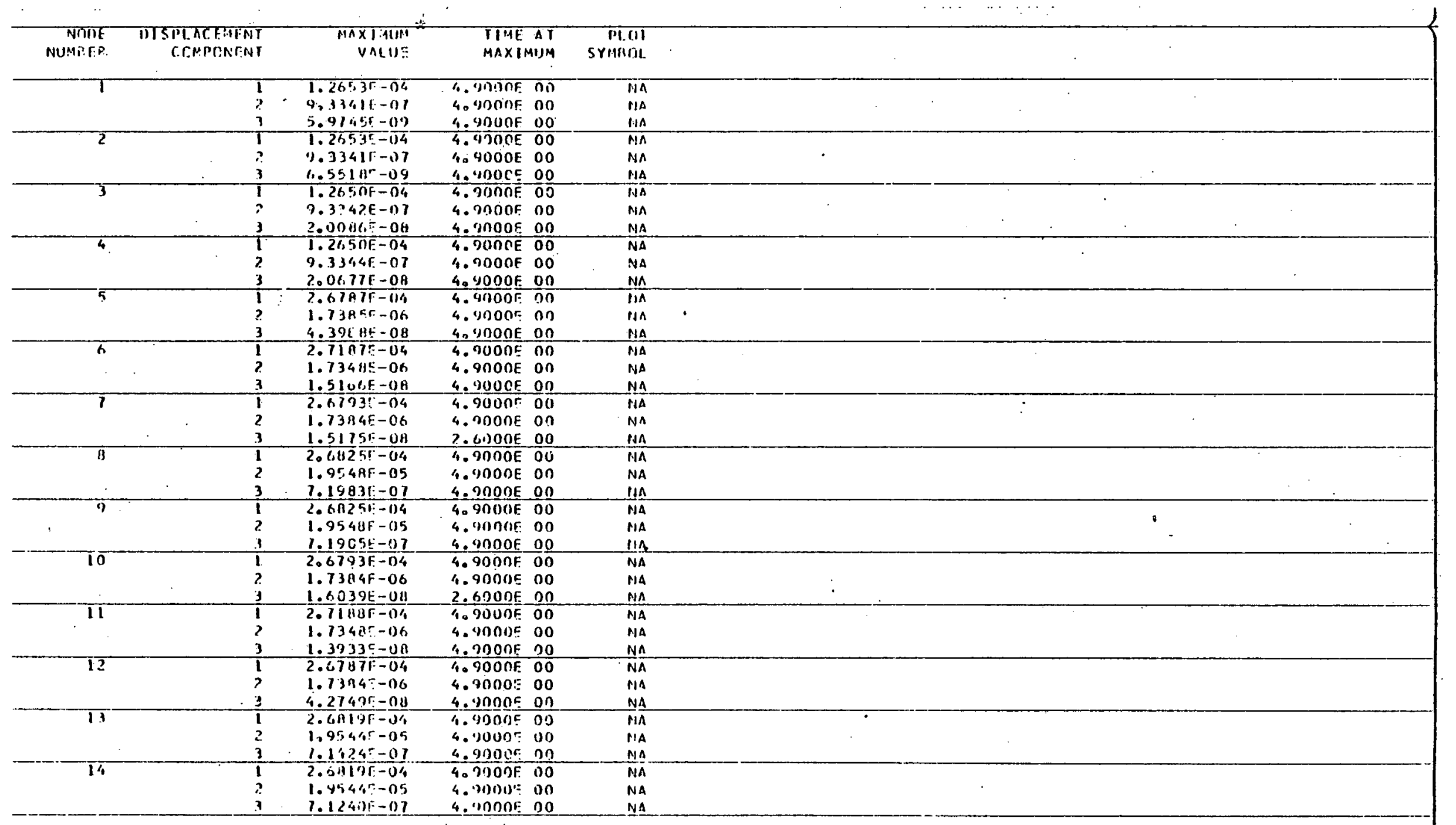

* A11 values represent the absolute maximum. 


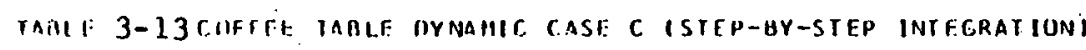

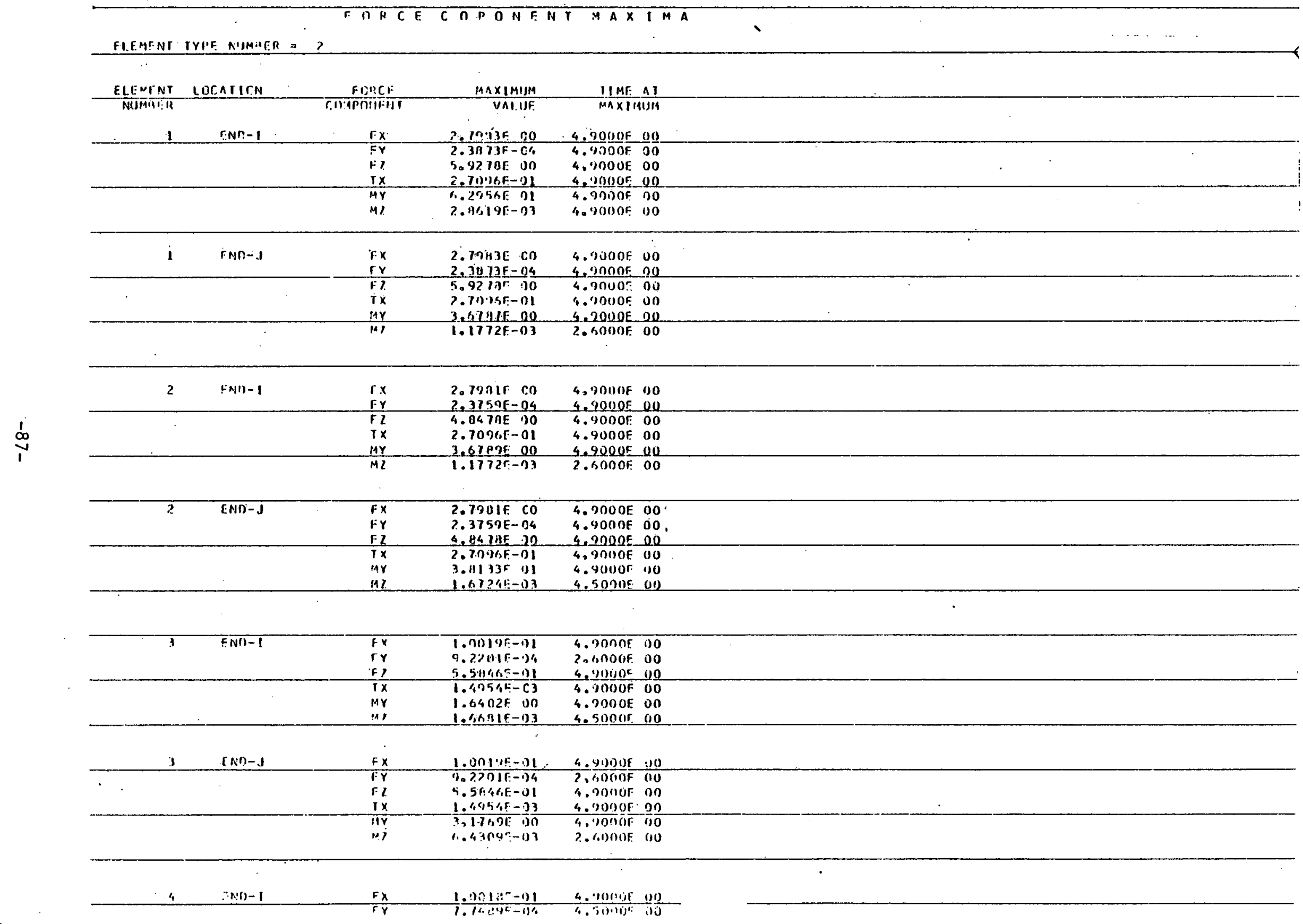




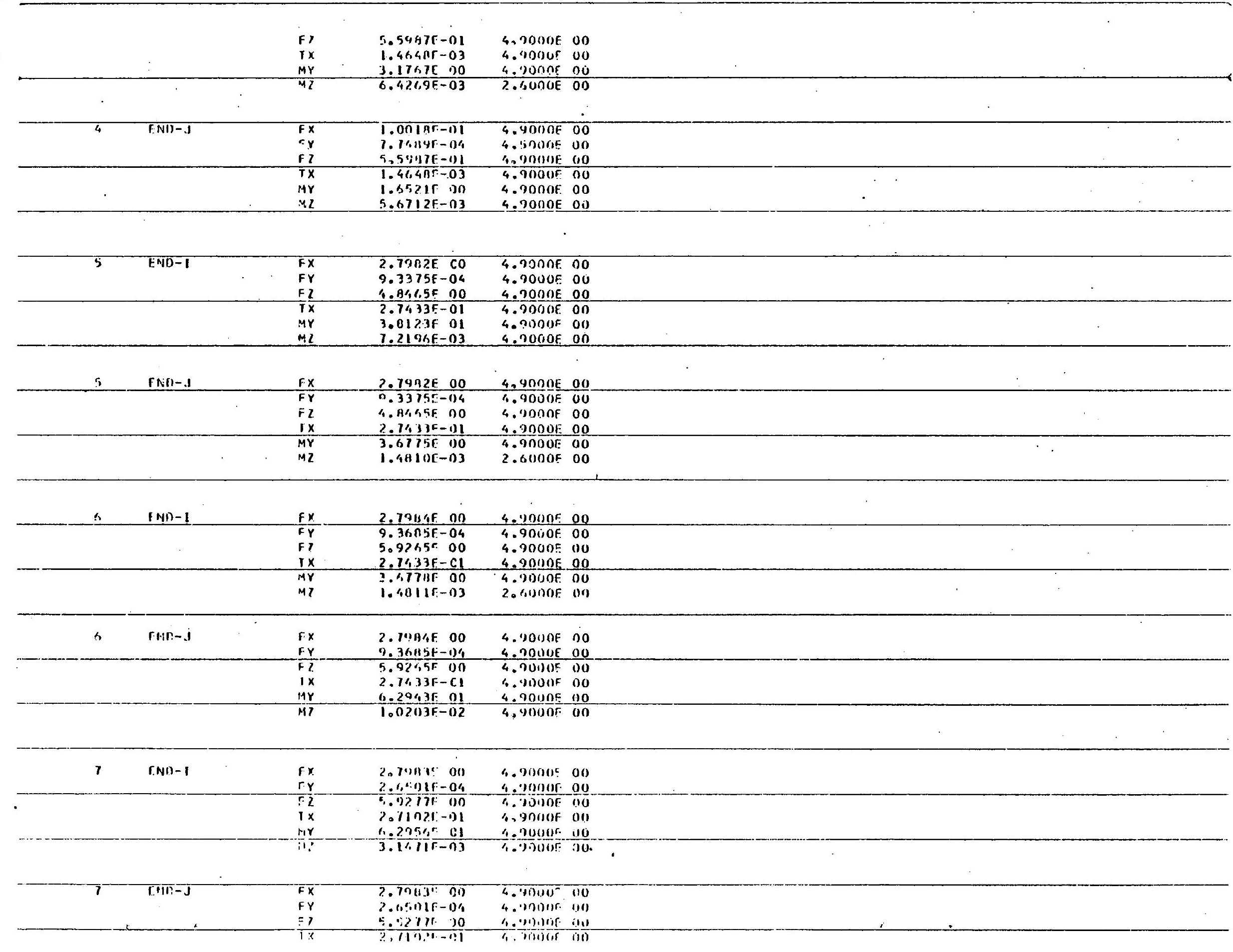




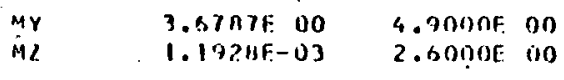

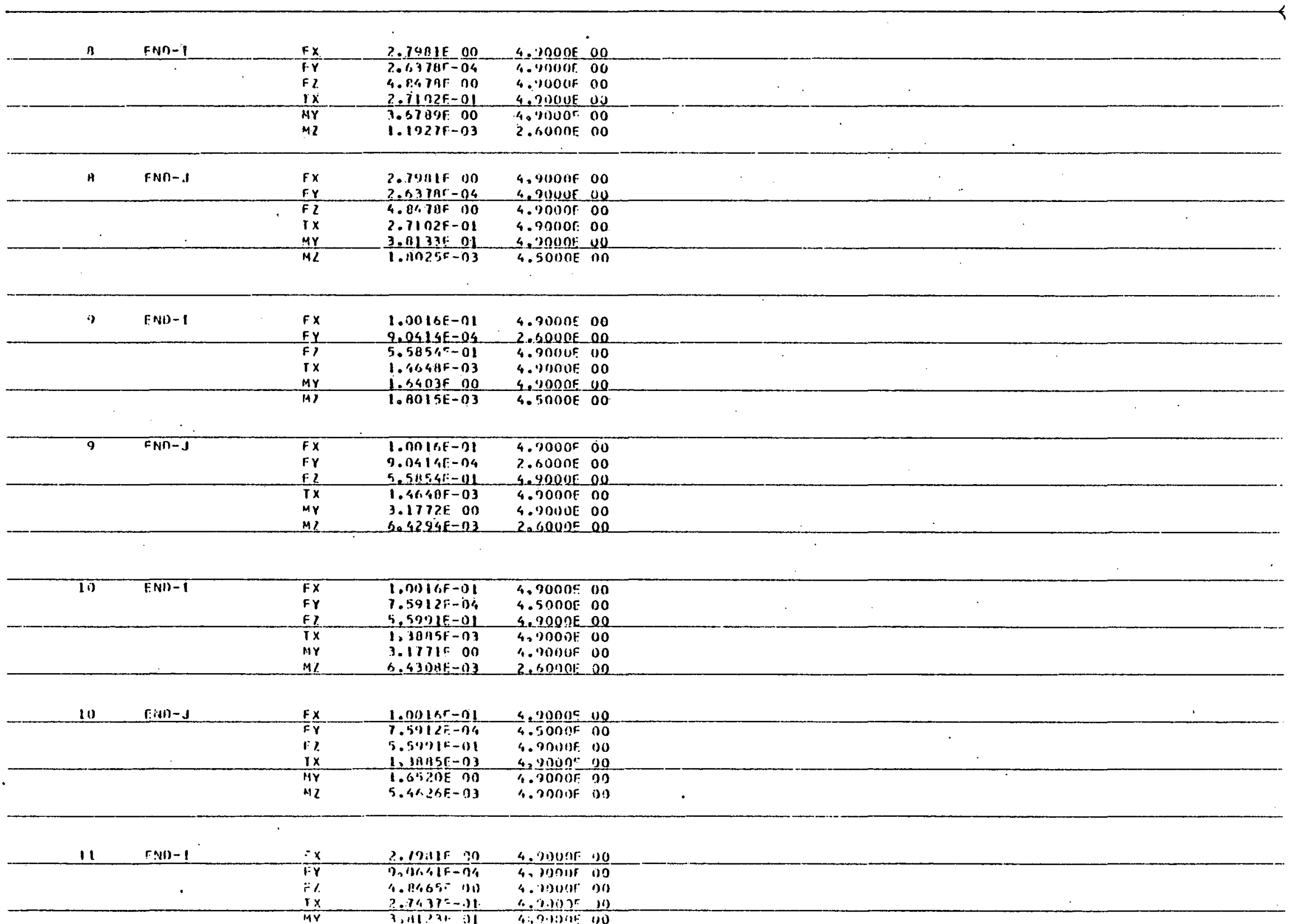




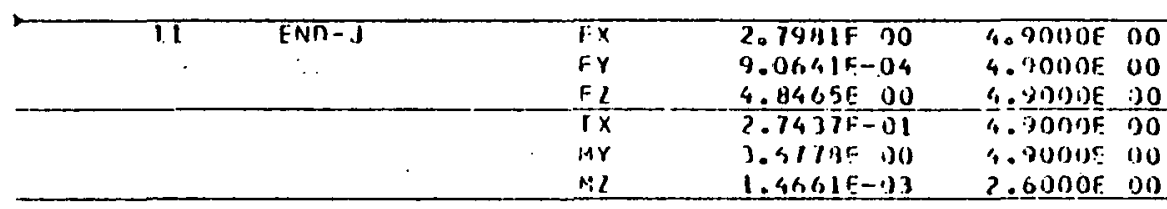

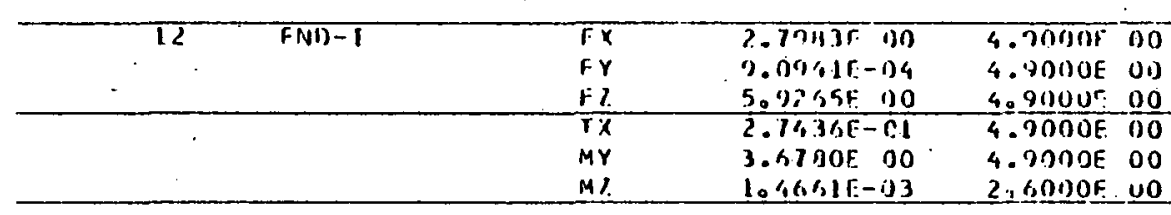

\begin{tabular}{|c|c|c|c|c|}
\hline 12 & I:NO-. & $F X$ & 2. ponar co & 4.20010 ก0 \\
\hline & & $F Y$ & $9.09216-04$ & $4.4000 E 00$ \\
\hline & & F. 2 & $5097,651: 20$ & $4.91100 E$ UO \\
\hline & & I $\underline{x}$ & $2.7436 F-01$ & 4,20000 on \\
\hline & & $\begin{array}{l}\overline{M r} \\
M V\end{array}$ & 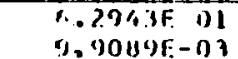 & $\begin{array}{l}4.9000 \mathrm{OF} \text { un } \\
6,4000 \mathrm{~s}\end{array}$ \\
\hline
\end{tabular}

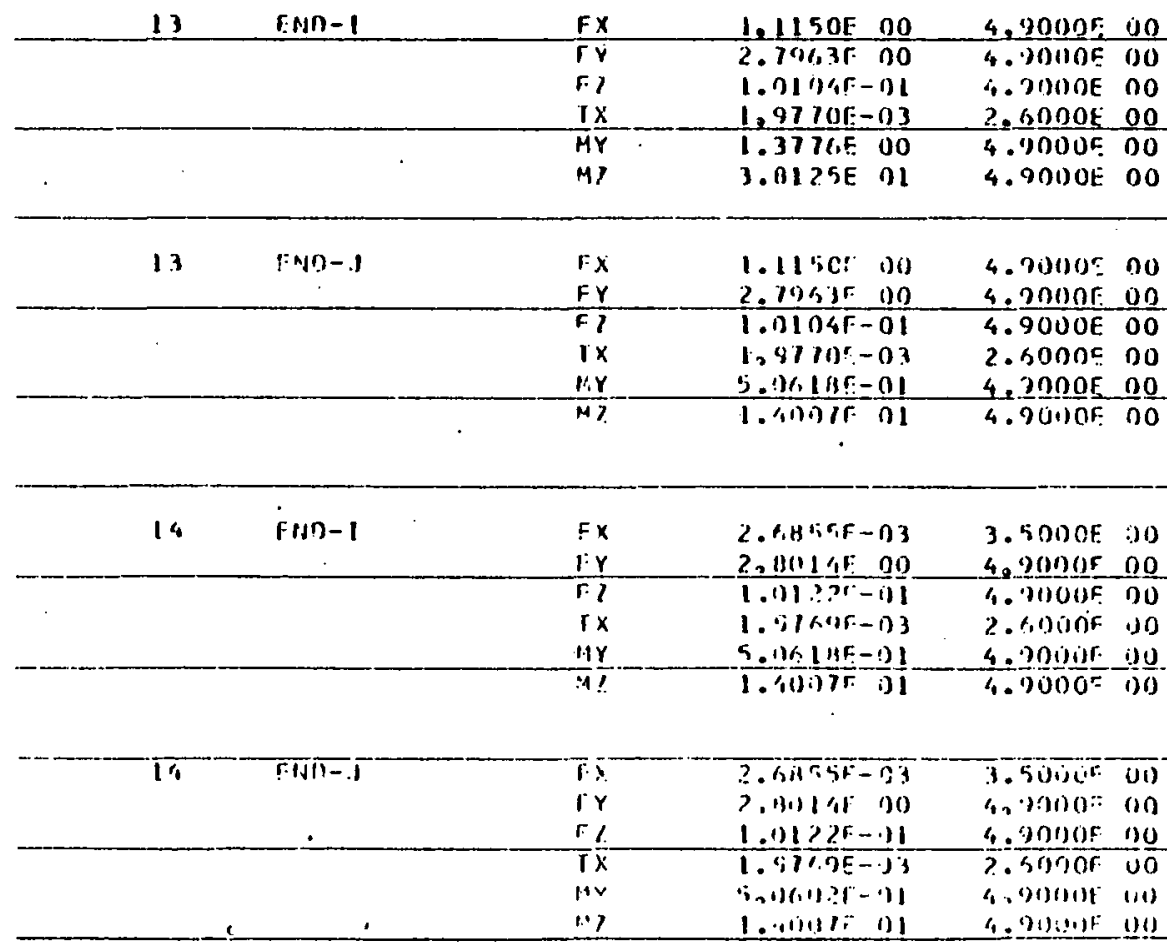




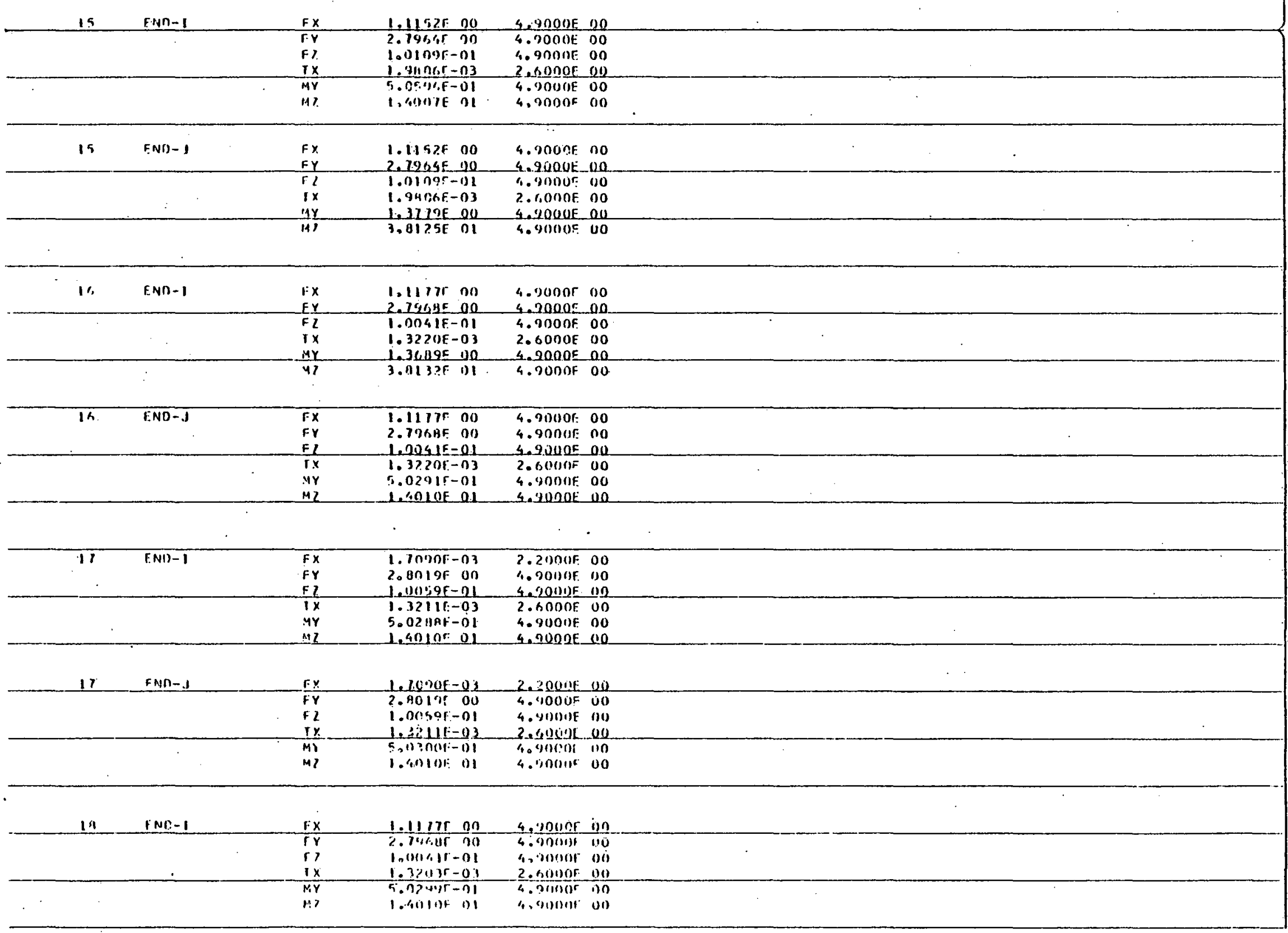


in FND-1

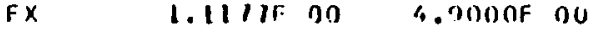

2.79681: OO $4.900 \mathrm{OJF} 00$

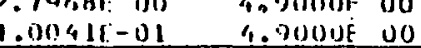

$\frac{1.00418-01}{1.321) 35-03}-\frac{1.9000 \mathrm{f}}{2.500}$

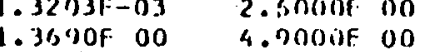

3.81321:01 4.9000r 00 


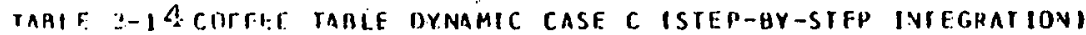

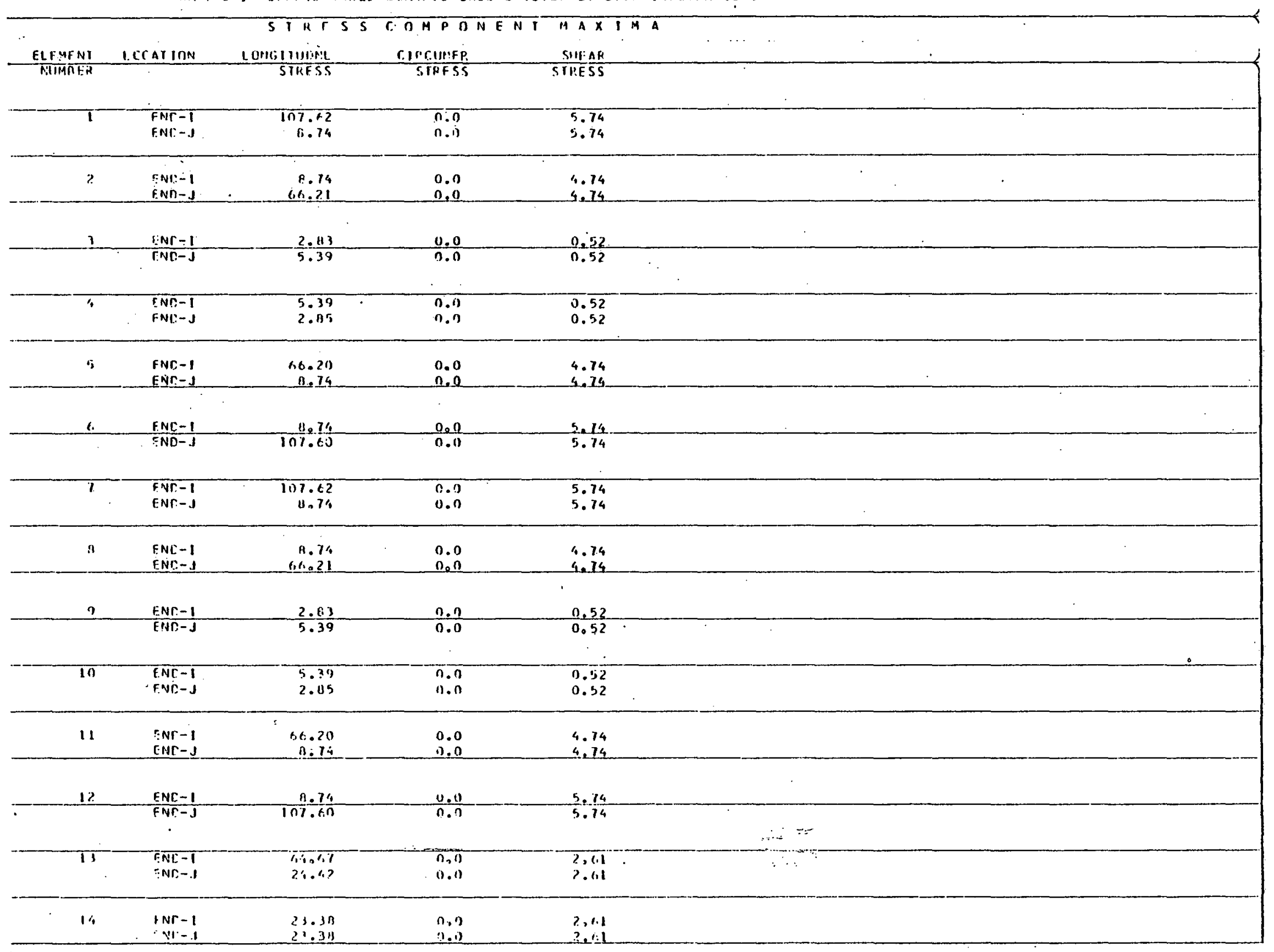




\begin{tabular}{|c|c|c|c|c|}
\hline 15 & $\begin{array}{l}\text { ENI:-1 } \\
\text {-NIT-J }\end{array}$ & $\begin{array}{l}21.48 \\
64.67 \\
\end{array}$ & $\begin{array}{l}0.0 \\
0.0 \\
\end{array}$ & $\begin{array}{l}2.61 \\
2.61 \\
\end{array}$ \\
\hline 16 & $\frac{\text { ENT-1 }}{\text { ENT:-D }}$ & $\frac{64.69}{240.42}$ & $\frac{0.0}{n, n}$ & $\frac{2.61}{2.6 .1}$ \\
\hline 17 & $\begin{array}{l}\text { FNF -1 } \\
\text { ENT -.. }\end{array}$ & $\begin{array}{l}23.34 \\
23.39\end{array}$ & $\begin{array}{l}0.0 \\
0.0\end{array}$ & $\begin{array}{l}2.61 \\
2.661\end{array}$ \\
\hline 18 & $\begin{array}{l}\text { ENr - - } \\
\text { ENC - }\end{array}$ & $\begin{array}{l}24.42 \\
64.69 \\
\end{array}$ & $\begin{array}{l}0.0 \\
0.0 \\
\end{array}$ & $\begin{array}{l}2 .+1 \\
2,61\end{array}$ \\
\hline
\end{tabular}


Problem 4 - Static Analysis of Spence's Pipe Work Problem

In this problem, a piping system provided by spence

was analyzed. Comparisons are made between results obtained

from the 'EPIPE' program and those obtained by spencell, from the SPANDIE program.

\section{Problem Description}

i. Geometry - The layout of this piping system is shown in Fig. 4-1. The piping elements 2-3 and 10-11 are stiffened by taking the internal diameter of the pipe as zero. Table 4-1 shows the input nodal coordinates data. The element properties of the pipe are as follows:

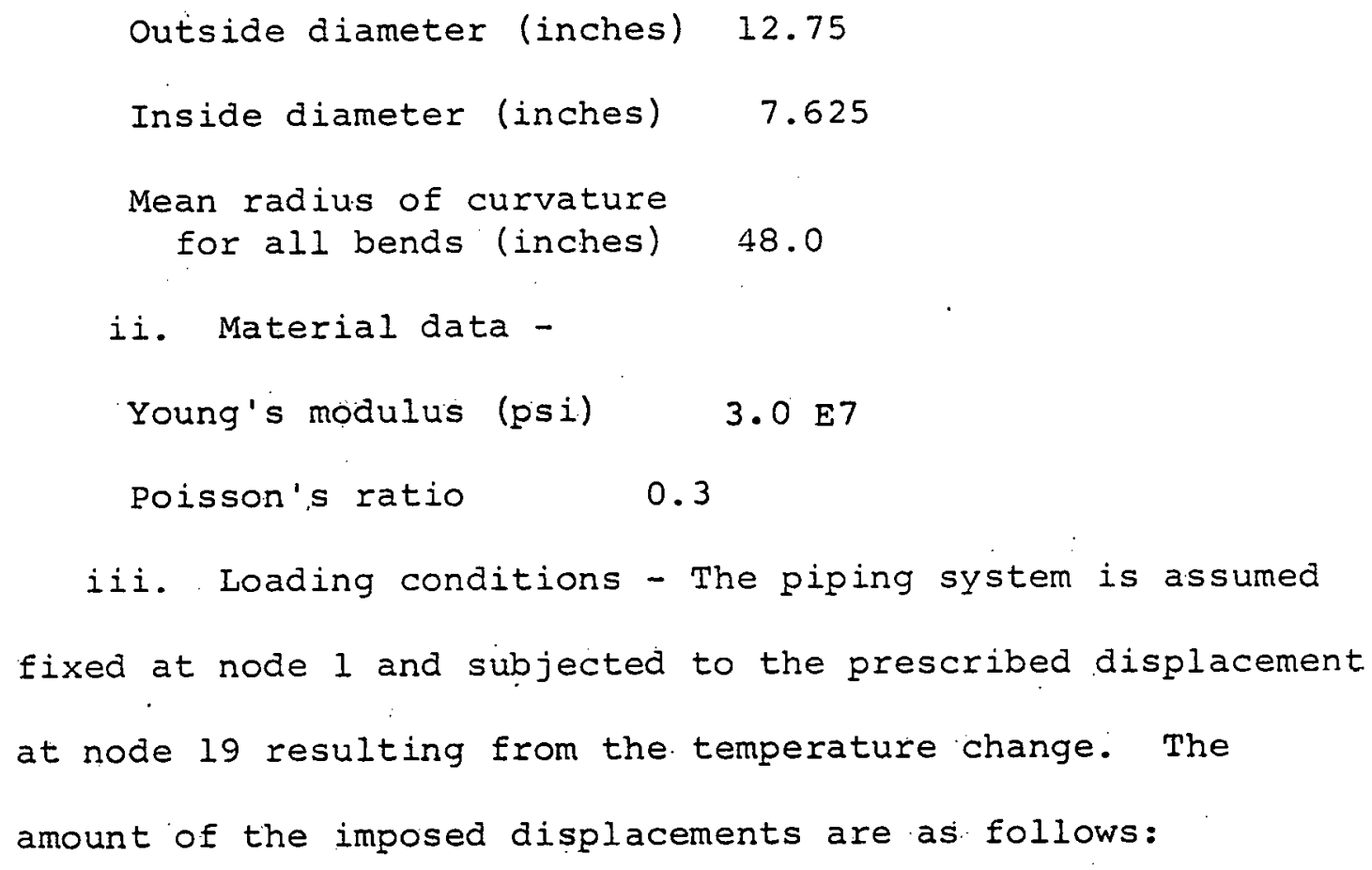




$$
\begin{aligned}
& x-\text { displacement }=6.8526 \mathrm{in} . \\
& y-\text { displacement }=1.9602 \mathrm{in} \\
& z \text { - displacement }=2.6487 \mathrm{in} . \\
& \text { Analysis Results }
\end{aligned}
$$

The computer output is given in Tables 4-2 to 4-4, showing the nodal displacements/rotations, pipe forces and moments, and pipe stresses respectively. It is to be noted that due to extreme bending different fiber stresses result at the same crosssection of the tubes. These ard shown as the positive and negative values given in Table 4-4. A comparison of the results are made on a selected number of nodes and elements as shown in Tables 4-5 to 4-6. The results obtained from the EPIPE agree quite well with those obtained from SPANDLE. 


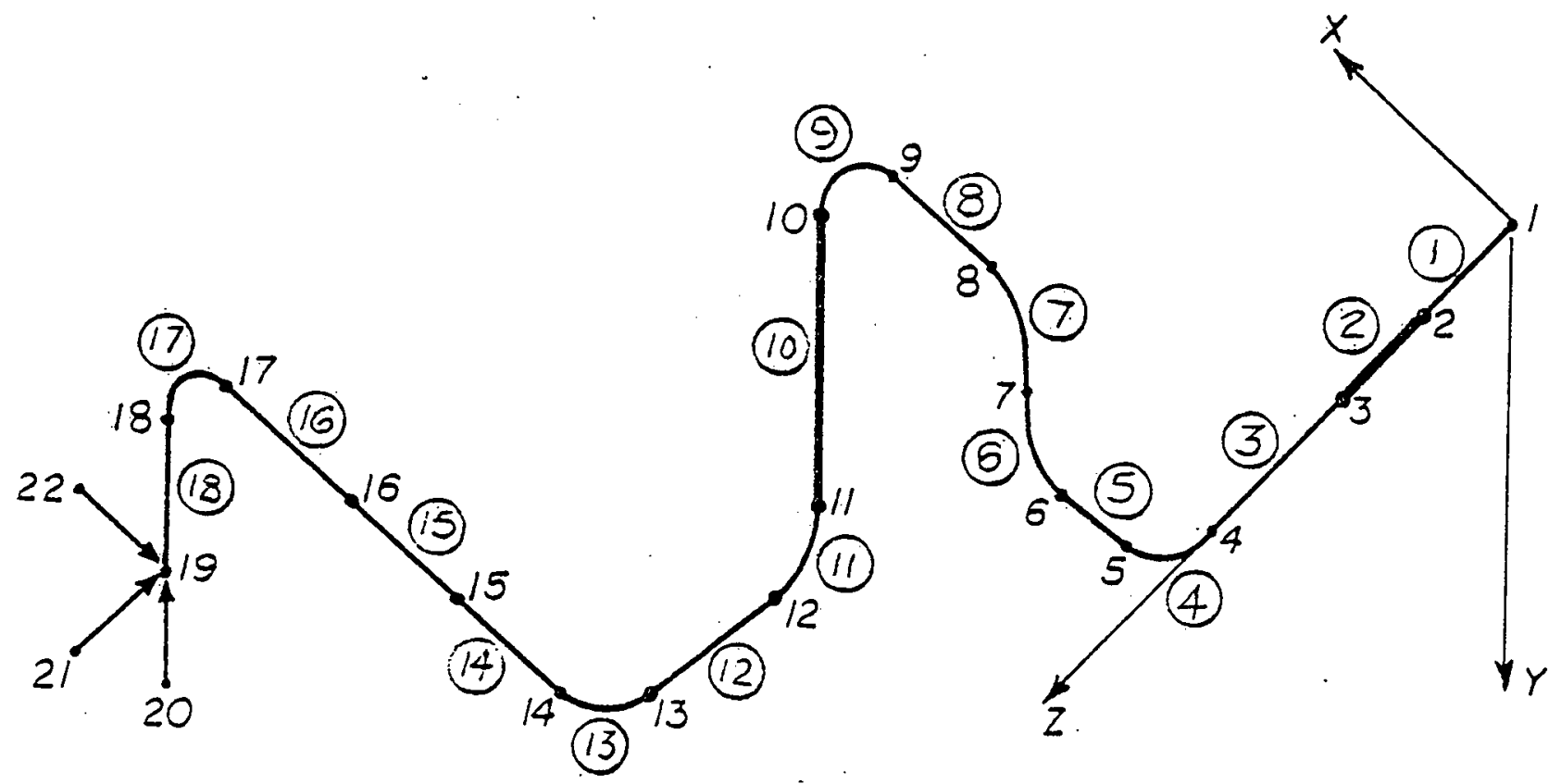

$$
\begin{gathered}
\text { Fig. } 4-1 \\
\text { Isometric Sketch of Spence's Problem }
\end{gathered}
$$




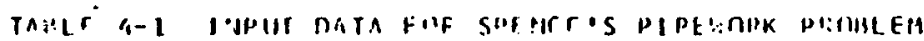

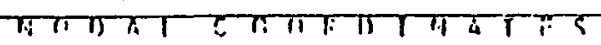

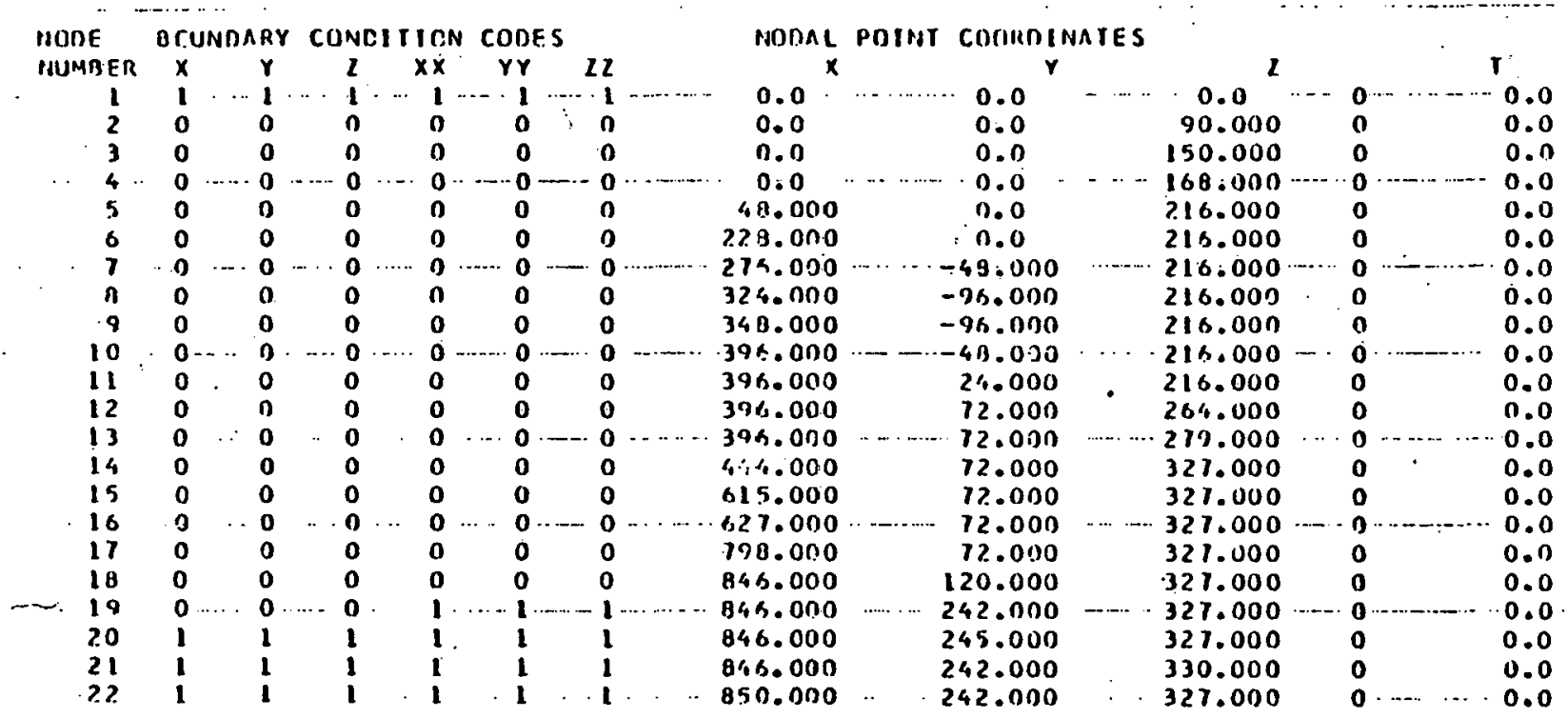

1
$\infty$
1 


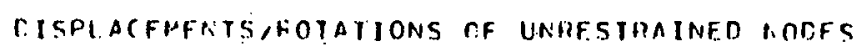

\begin{tabular}{|c|c|c|c|c|c|c|}
\hline $\begin{array}{l}\text { NOINF } \\
\text { RIJPISER }\end{array}$ & TFANSLAIION & IFANSLATION & THANSLATIONN & DOInTION & vetation & hotarion \\
\hline$?$ & $-5.5 \times 334 \mathrm{~F}=01$ & $-1.5374>E-01$ & -3.0 KA?? \&F. -04 & $3 . \ln 3125-03$ & - ] O OF 4 PQE - O? & $-4.97600 F_{-}-03$ \\
\hline 3 & $-1.26 G 11 E+n n$ & $-3.72 ? ? .3 \mathrm{E}-01$ & $-5.25909 F-04$ & $4 . \cap A 3 H \cap F-\cap 3$ & $-1.2219 n E-0 ?$ & $-7 . A 7042 F-03$ \\
\hline 4 & -1. AP55TE+ ON & $-4.4 A \& 15 E-n 1$ & $-5.99554 E-04$ & $4.27395 f-03$ & $-1.193 ? 5 E-0 ?$ & $-P . P+5 A O F-\cap 3$ \\
\hline 5 & $-7, n \cap 12 \sigma E+O N$ & $-1.1 \subseteq 40 I E+00$ & $4.536855-01$ & 4.1634 กf. -03 & JOAOE-O3 & $-1 . ? 2469 F-02$ \\
\hline 6 & $=2.0 \cap 4 \backslash 4 E+0 \cap$ & $-? .7 \div 315 E+10$ & 9.975 AAF-01 & $3.93544 E-03$ & -3.7 .31 POE -05 & $-1.46 .915 E-n ?$ \\
\hline 7 & $-? . E E .16 E E+00$ & $-4.4 n 949 E+00$ & e. $011533 F-01$ & $3.25691 E-03$ & $5.277915 .-04$ & -1.79 \\
\hline 4 & $-3.13 \cap 07 E+0 \cap$ & $-4.7+45.3 E+0 n$ & $6.5379 ? F-01$ & $1.1 n 56: 1 E-03$ & $3.95750 E-04$ & $-3.94996 E-\cap 3$ \\
\hline 9 & $=3.13 n 45 . E+0 n$ & $-4 . A 1692 E+00$ & $6.4 \mathrm{AP} 30 \mathrm{OF}-\mathrm{nI}$ & $1.744 \mathrm{~A} 3 \mathrm{E}-04$ & $-3.2 A+9, E-D K$ & $-4 .+5$ H? 3F - 04 \\
\hline 10 & $-3.4 \div 58$ & $-A, A \leq 3 A I E+O N$ & $6.12935 \mathrm{~F}-01$ & $-2.21525 F=03$ & $-2.75455 E-03$ & $4 a F-03$ \\
\hline 11 & $-4.279305+00$ & $-4.6540 ? F+0 n$ & $225-01$ & $-2.57212 \mathrm{k}-03$ & -5.516 & 1.???7HE- - 2 \\
\hline 1? & $-5 .\ulcorner 5090 E \div 00$ & $-4.55540 E+00$ & $3.1 A 596, F-01$ & $-1.7 .3394 E-03$ & $-7.45 P \cap 7 E-0.3$ & 1.05 ? R OE - त? \\
\hline 13 & $-5.37 n 50 F+00$ & $-4.5306 / E+0 n$ & $3.185345-01$ & $-1 . h \cap 9.31 E-03$ & $-7.0 .340 .3 E-03$ & $.006365-02$ \\
\hline 14 & $-5 . t .3365 t+n n$ & $-4.0 P 7 \cap ? E+00$ & $5.0721 .3 F-n 1$ & $-1.89972 F-03$ & $-1.71419 E-03$ & $.5[2] 4 E-103$ \\
\hline 15 & $-5 . t 3643 \mathrm{E} \cdot 0 \mathrm{n}$ & $-? .>$ S P O $\cap F+0 n$ & $-1.5563 P F-01$ & $-2.54149 E-03$ & 7.9787 OE -03 & $.7 .74952 \mathrm{~F}-\mathrm{n3}$ \\
\hline 16 & $-5.6365 \in E+0 n$ & $-3.25376 E+00$ & $-2.53433 F-01$ & -2. GAGP 9F. - 03 & 9. $.2 A T A A E-0.3$ & $3.70076 E-03$ \\
\hline 11 & $-5.679 .33 \mathrm{~F}+0 \mathrm{n}$ & $-\bar{\varepsilon} .37290 E+00$ & $-1.8 \geq 3 \cap 4 E+00$ & -3.4 ?AGGE-03 & $8.69 S \cap 5 E-03$ & $7.57431 \mathrm{E}-03$ \\
\hline 18 & $-K, O A>C, 1 E+O \cap$ & $-1.959 .85 .+00$ & -2.3 . 54 SE. $+0 \cap$ & $-.7 .5 .37\} 95-03$ & 5. HQP 7 D.E -0.3 & $9.52453 F-03$ \\
\hline 10 & $-6.95 ? 600+00$ & $-1.9 E n \geq 0 E+0 n$ & $-7.64 B 70 F+00$ & 0 & n & $n$ \\
\hline
\end{tabular}




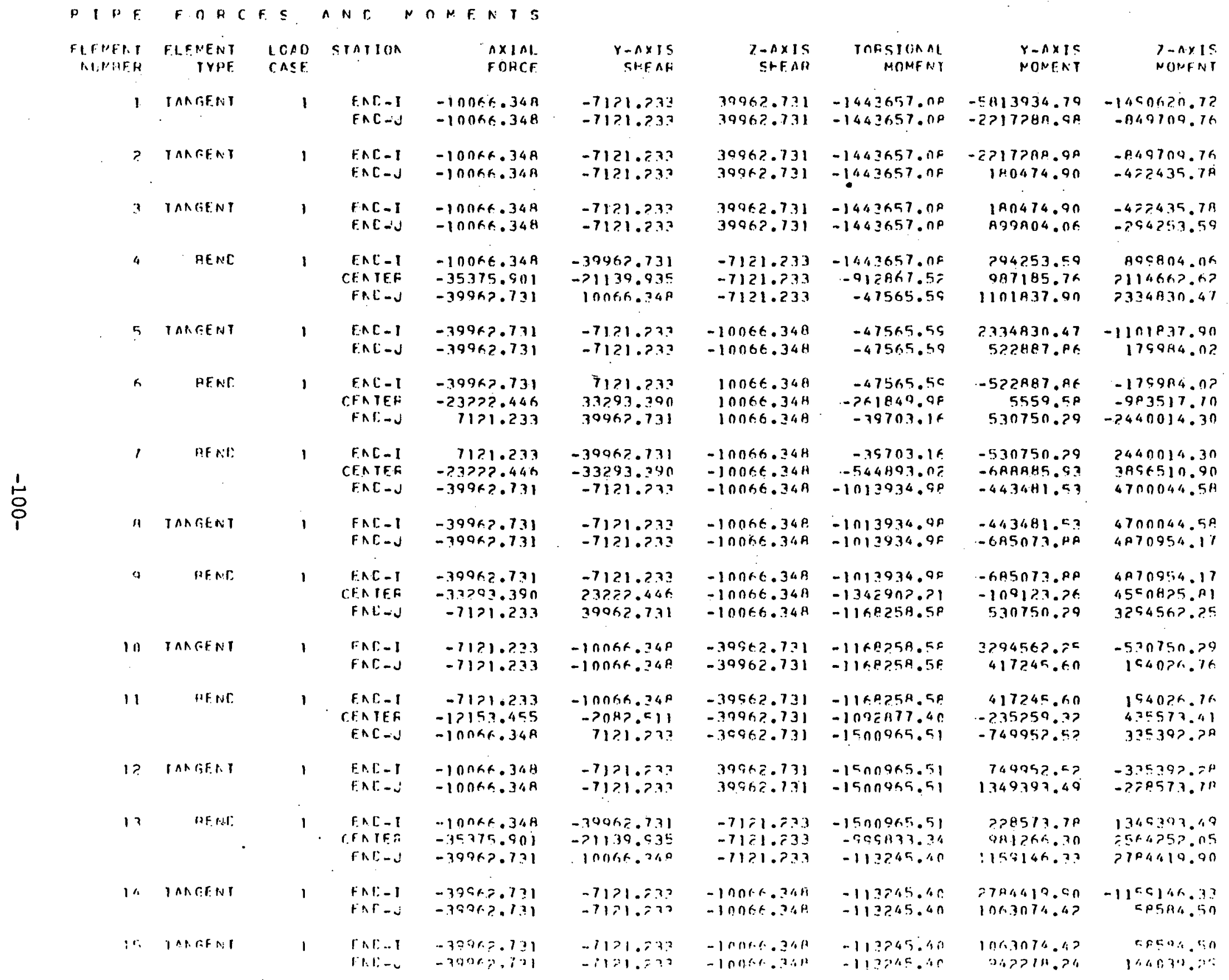


TAULF 4-? AAMI.YSIS OF SFFACENS PIPIAG SYSTEM

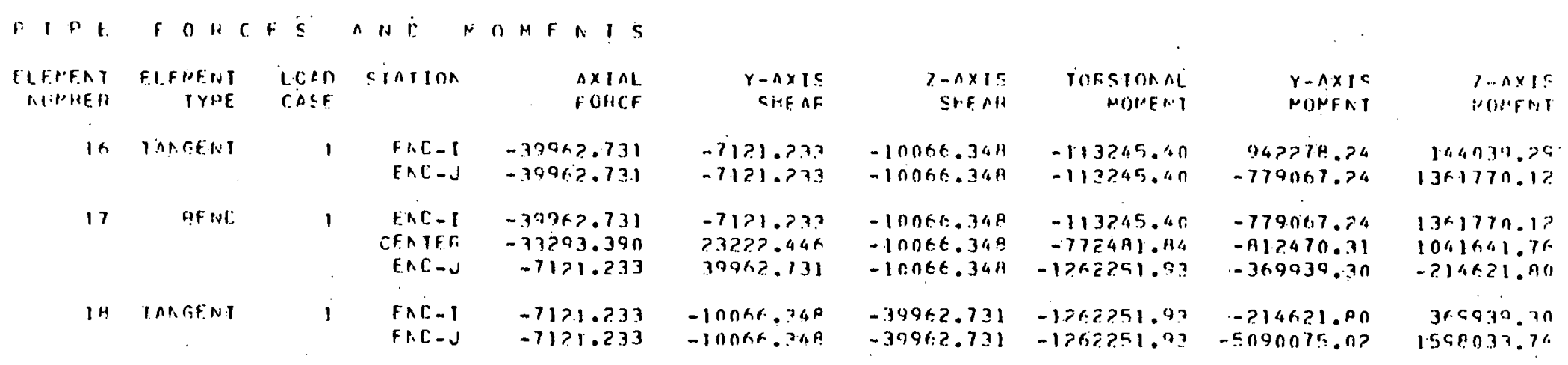




\begin{tabular}{|c|c|c|c|c|c|c|}
\hline & $\begin{array}{l}\text { FIFUAT } \\
\text { hHARK }\end{array}$ & $\begin{array}{r}\text { FLFWFNT } \\
\text { TYFF }\end{array}$ & $\begin{array}{l}1 . c \wedge n \\
r A \leq r\end{array}$ & STAIIna & $\begin{array}{l}\text { I. CNGITHOIHA } \\
\text { PCSITIVF }\end{array}$ & $\begin{array}{l}\text { STHFSS } \\
\text { MESinilive }\end{array}$ \\
\hline & 1 & IANGENI & 1 & $\begin{array}{l}\text { FAl: }-1 \\
\text { FAl:- }\end{array}$ & $\begin{array}{l}336.29 .70 \\
13250.27\end{array}$ & $\begin{array}{l}-3.3945 .10 \\
-1.3503 .70\end{array}$ \\
\hline & ? & $T A R$ TER T & $\mathbf{I}$ & $\begin{array}{l}\operatorname{EnC}-I \\
\operatorname{FAC}-J\end{array}$ & $\begin{array}{r}11590.51 \\
2.178 .69\end{array}$ & $\begin{array}{r}-11740.14 \\
-2336.3 \mathrm{H}\end{array}$ \\
\hline & 3 & I IAnA:FA:I & 1 & $\begin{array}{l}\text { E.t. } C-I \\
\text { EAC }-J\end{array}$ & $\begin{array}{l}2465.92 \\
5212.04\end{array}$ & $\begin{array}{l}-2711.40 \\
-5457.57\end{array}$ \\
\hline & 4 & DFAr. & 1 & $\begin{array}{l}\text { EAL }-I \\
\text { CEAIFF } \\
\text { FAL-J }\end{array}$ & $\begin{array}{r}5712.09 \\
12719.76 \\
14761.49\end{array}$ & $\begin{array}{r}-5457.57 \\
-135.97 .46 \\
-150.36 .04\end{array}$ \\
\hline & $<;$ & TANEENT & 1 & $\begin{array}{l}\operatorname{fac}-1 \\
\text { fac }-\mathrm{J}\end{array}$ & $\begin{array}{r}14061.49 \\
26.28 .9 R\end{array}$ & $\begin{array}{r}-15036.04 \\
-360 ? .53\end{array}$ \\
\hline & G & PFAC & 1 & $\begin{array}{l}\text { EAL }-I \\
\text { CFAIFF } \\
\text { EAC }-2\end{array}$ & $\begin{array}{r}2679.9 A \\
5259.26 \\
14159.36\end{array}$ & $\begin{array}{r}-3603.53 \\
-5 \% ? 5.54 \\
-1.39 \text { S. }^{-70}\end{array}$ \\
\hline & 7 & "urer. & 1 & $\begin{array}{l}\text { FAL }-1 \\
\text { CFA IFF } \\
\text { FAC-J }\end{array}$ & $\begin{array}{l}14158.36 \\
22015.03 \\
26116.12\end{array}$ & $\begin{array}{l}-1.3994 .7 n \\
-22591.25 \\
-27090.67\end{array}$ \\
\hline & $"$ & TAARENI & 1. & $\begin{array}{l}\text { tac- } \\
6 \cdot c-1\end{array}$ & $\begin{array}{l}26116.12 \\
27231.74\end{array}$ & $\begin{array}{l}-27090.67 \\
-28206.79\end{array}$ \\
\hline & 4 & UF AC & 1 & $\begin{array}{l}\text { ERL-I } \\
\text { CERIER } \\
\text { FAL-J }\end{array}$ & $\begin{array}{l}? 7231.74 \\
25244.29 \\
18718.10\end{array}$ & 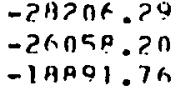 \\
\hline & $1 n$ & IAACENT & 1. & $\begin{array}{l}\text { FAC-I } \\
\text { Fac }-J\end{array}$ & $\begin{array}{r}1971 A .10 \\
7506.23\end{array}$ & $\begin{array}{r}-18 \times 91.76 \\
-2679.49\end{array}$ \\
\hline & 11 & RENC & 1 & $\begin{array}{r}\text { EAC-I } \\
\text { RARTER } \\
\text { ARI:-J }\end{array}$ & $\begin{array}{l}2506.23 \\
2641.5 n \\
4509.77\end{array}$ & $\begin{array}{l}-2679.84 \\
-2931.89 \\
-1752.26\end{array}$ \\
\hline & 17 & $T \wedge A F F N T$ & 1 & $\begin{array}{l}{[A:-1} \\
\operatorname{cac}[-]\end{array}$ & $\begin{array}{l}4506.77 \\
75,19.7 n\end{array}$ & $\begin{array}{l}-4757.86 \\
-79.35 .14\end{array}$ \\
\hline & 1.3 & PFP:C: & 1 & $\begin{array}{l}\text { FAC - I } \\
\text { CFATFE } \\
\text { FAC - }\end{array}$ & $\begin{array}{l}74119.7 n \\
15010.65 \\
\text { IGSOA.AB }\end{array}$ & $\begin{array}{r}-7 a 75.111 \\
-15902.34 \\
-174119.111\end{array}$ \\
\hline & 11 & $1 \cap A C: F A: 1$ & 1 & $\begin{array}{l}\text { F.C. }-1 \\
\text { Fac... }\end{array}$ & $\begin{array}{l}1+509.185 \\
5: 18.047\end{array}$ & $\begin{array}{r}-176113.111 \\
-1.4117 .011\end{array}$ \\
\hline
\end{tabular}

\begin{tabular}{|c|c|c|c|}
\hline hicer & SIfits? & \multicolumn{2}{|r|}{ SIDFs: } \\
\hline FosITIUf. & AFGAIIUF & IECSITJVF & AF(INIIVE. \\
\hline $0 . n n$ & $0 . c .0$ & -3577.71 & $-65 f_{1}>0.1$ \\
\hline n. no & 0.00 & -3577.71 & $-4.5 k>.+1$ \\
\hline$n .0) n$ & 0.00 & $-2 ? 29.4 ?$ & $-3 \mu K 5.2 H$ \\
\hline 0.00 & $0 . n n$ & -.328 .9 .47 & $-34 \times 5.2 A$ \\
\hline$n . n n$ & $0.0 n$ & $-357>.71$ & $-454 ? .61$ \\
\hline $0.0 n$ & 0.00 & -3577.71 & $-45 h ? \cdot+1$ \\
\hline $105 \% .4 \%$ & $-213 \mu .5 \beta$ & -.2577 .71 & $-4 \vdots \xi>.+1$ \\
\hline 2447.43 & -5075.45 & $-2.3 n n .11$ & $-2.844 \cdot 1 \cdot n$ \\
\hline 2.746 .01 & -5545.82 & 16.33 & $-74 \% .37$ \\
\hline n. & 0.00 & 14.3 .3 & $-2.94 . .77$ \\
\hline 0.00 & $n .0 n$ & 16.33 & $-284 . .77$ \\
\hline 211.71 & -427.77 & 16.3 .3 & -2.94 .37 \\
\hline 1356.135 & -23.37 .54 & -31.69 & $-\mid 161.89$ \\
\hline 2870.14 & -5795.81 & 390.63 & -614.36 \\
\hline $2 P 7 n .14$ & -5795.81 & 290.63 & -614.36 \\
\hline $45 A 3.38$ & $-9 ? 50 . P G$ & -1.111 .10 & -1859.40 \\
\hline $552 P .56$ & $-11170.6 \%$ & -27 in .57 & $-3 \cap 07 . ? ?$ \\
\hline$n .0 n$ & 0.00 & $-2.7 n+.5 ?$ & $-3 n n 7.7 ?$ \\
\hline$n .00$ & 0.00 & $-87 n \pi .5 ?$ & $-3 n n 7.27$ \\
\hline 5729.60 & $-1157 t . P A$ & $-270 x .5 ?$ & -30n7.77 \\
\hline 5.353 .14 & -IORIt. . II & -3475.16 & $-4 \cap 92.3 A$ \\
\hline 3475.72 & $-78.3 n .83$ & $-27 \times 0.20$ & -.3704 .19 \\
\hline 0.00 & $n, n n$ & -7790.20 & $-3794,19$ \\
\hline $0.0 n$ & $0 . n n$ & -2790.20 & -3794.10 \\
\hline $220.8 ?$ & $-4 h 1.15$ & -2790.20 & -3794.19 \\
\hline $51 \overline{2.24}$ & - In & $-2591 . .36$ & $-356.7 . ?$ \\
\hline I94.594 & -797.17 & -27.74 .18 & $-47>4.08$ \\
\hline$n \cdot n n$ & $n . \cap n$ & -3734.18 & $-4724,08$ \\
\hline 0.00 & 0.00 & -.2734 .18 & $-47>4 . \cap A$ \\
\hline 19117.76 & -.7207 .17 & -.7734 .19 & $-47>4.0 n$ \\
\hline $2 \cap 16 . \because 89$ & -6.0914 .119 & $-25,15.14$ & -2 - 91.13 \\
\hline $7=77^{\circ} .24$ & -6.617 .77 & -169.77 & $-460.4 ?$ \\
\hline$n \cdot 1: n$ & $0 . n$ & 1.8 .73 & c.e \\
\hline$n .1 ! n$ & $n \cdot(n)$ &. .140 .73 & $-10,0$. \\
\hline
\end{tabular}


IMIIIF A-A AAMLYSIS OF SHFACEIS PIIING SYSTEM

F I V I: S T B E S S F S

\begin{tabular}{|c|c|c|c|c|c|}
\hline 15 & TAARFNI & 1 & $\begin{array}{l}\text { EAL }-1 \\
\text { FAC.-J }\end{array}$ & $\begin{array}{l}1910.29 \\
1.371 .51\end{array}$ & $\begin{array}{l}-5545.70 \\
-4997.51\end{array}$ \\
\hline $1 \%$ & IAAREFAT & 1 & $\begin{array}{l}\text { EAC-V } \\
\text { EAL-1 }\end{array}$ & $\begin{array}{l}49 A 4.34 \\
H 35.7 .66\end{array}$ & $\begin{array}{l}-5 \text { - } 58.89 \\
-9378 . ? 1\end{array}$ \\
\hline 17 & WENR. & $i$ & $\begin{array}{l}\text { FAL - I } \\
\text { CEAIER } \\
\text { EAL-J }\end{array}$ & $\begin{array}{l}8.353 .66 \\
7038.34 \\
2323.24\end{array}$ & $\begin{array}{l}-932 n .71 \\
-7450.25 \\
-21496.95\end{array}$ \\
\hline $11 !$ & $T A M G F A$ & 1 & $\begin{array}{l}\text { EAC:-I } \\
\text { EAC-J }\end{array}$ & $\begin{array}{r}2.327 .20 \\
? 977.21\end{array}$ & $\begin{array}{r}-2496.55 \\
-30150.64\end{array}$ \\
\hline
\end{tabular}

\begin{tabular}{|c|c|c|c|}
\hline n. & $0.0 n$ & -181.09 & $-37 \therefore .044$ \\
\hline n. no & 0.00 & -181.69 & $-374.0 \%$ \\
\hline 0.00 & $0.0: n$ & $-1 \times 8.73$ & -460.4 .3 \\
\hline 0. no & $0.0 n$ & -168.73 & $-4 \times 9.43$ \\
\hline בR & $-3>36.54$ & -16.8 .73 & -469.4 .7 \\
\hline $1=25.26$ & $-2475.6 P$ & $-1 P \% 7.94$ & -2495.16 \\
\hline $757.4 \mathrm{f}$ & -510.09 & -3054.03 & $.4059 .0 ?$ \\
\hline$n .00$ & $0 . n n$ & -2054.03 & -405 \\
\hline$n .0 n$ & 0.00 & $-3 n 54.03$ &.-405 \\
\hline
\end{tabular}

占 
Table 4-5

Comparison of Nodal Displacements/Rotations

\begin{tabular}{lccccccc} 
Solution & Node & X-DISP & X-DISP & Z-DISP & XR & YR & ZR \\
EPIPE & 6 & -2.0041 & -3.7232 & 0.99358 & $3.8354 \mathrm{E}-3$ & $-3.2319 \mathrm{E}-5$ & $-1.4692 \mathrm{E}-2$ \\
Spence & 6 & -2.0044 & -3.7244 & 0.99407 & $3.8338 \mathrm{E}-3$ & $-3.4540 \mathrm{E}-5$ & $-1.4697 \mathrm{E}-2$ \\
EPIPE & 11 & -3.4259 & -4.6538 & 0.61294 & $-2.2153 \mathrm{E}-3$ & $-2.7546 \mathrm{E}-3$ & $3.2905 \mathrm{E}-3$ \\
Spence & 10 & -3.4265 & -4.6560 & 0.61375 & $-2.2179 \mathrm{E}-3$ & $-2.7548 \mathrm{E}-3$ & $9.2867 \mathrm{E}-3$ \\
\hline
\end{tabular}


Table 4-6

Comparison of Forces and Moments

\begin{tabular}{|c|c|c|c|c|c|c|c|}
\hline Solution & Element & FX & FY & $F$ & $\mathrm{TX}$ & MY & $M Z$ \\
\hline EPIPE & $\begin{array}{r}1-\mathbf{I} \\
-J\end{array}$ & $\begin{array}{l}10066 \\
10066\end{array}$ & $\begin{array}{l}39963 \\
39963\end{array}$ & $\begin{array}{r}7121 \\
-7121\end{array}$ & $\begin{array}{r}1443657 \\
1443657\end{array}$ & $\begin{array}{l}-1490620 \\
-849709\end{array}$ & $\begin{array}{r}5813935 \\
-2217288\end{array}$ \\
\hline Spence & $\begin{array}{r}1-I \\
-J\end{array}$ & $\begin{array}{l}10065 \\
10065\end{array}$ & $\begin{array}{l}39963 \\
39963\end{array}$ & $\begin{array}{r}7123.7 \\
-7123.7\end{array}$ & $\begin{array}{l}1444200 \\
1444200\end{array}$ & $\begin{array}{l}-1490900 \\
-849790\end{array}$ & $\begin{array}{r}5814300 \\
-2217600\end{array}$ \\
\hline
\end{tabular}


Problem 5 - DYNAMIC RESPONSE OF A LOW FREQUENCY COFFEE TABLE

It is important to realize that there can be greater differences between the response spectrum results and those obtained from either time-history or direct integration methods, when the natural frequencies of the system are in the vicinity of the peak earthquake values. To demonstrate this, the legs of the coffee table (problem 3) were extended so the natural frequencies come out to be in the vicinity of the earthquake peak shown in Figure 2-3. As can be seen by comparing results shown In Tables 3-7 and 3-11 with those of Tables 5-7 and 5-10, there are indeed greater differences (about three to one) between the response spectrum and the time history and direct integration method results for the case where the coffee table's natural frequencies fall out near the peaks. 
TAALE 5-1-LOW-PFEOUENGY-CCFFEE-TAALE-CASE-A-ITIME HISTOAY-ANALYSISI

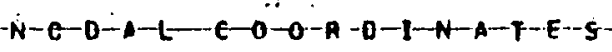

NODS HOUNOAQY CONOIYION COUES

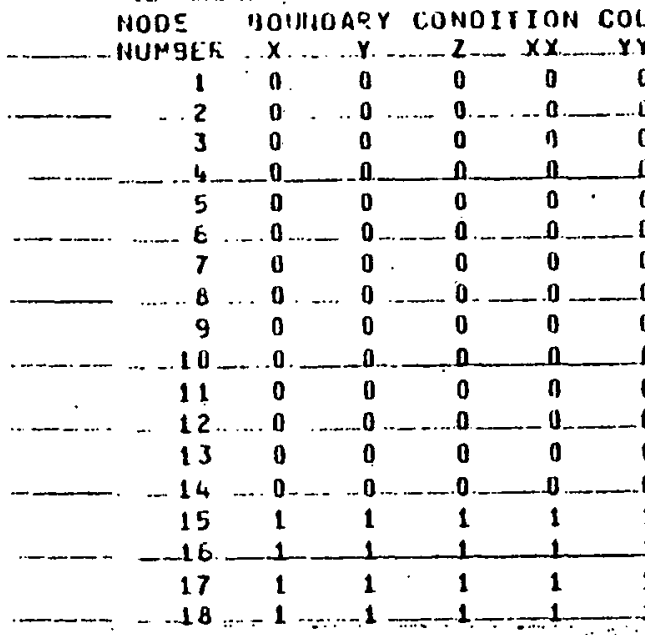

\begin{tabular}{lr}
0 \\
0 \\
0 & 0 \\
0 & 0 \\
0 & 0 \\
0 & 0 \\
0 & 0 \\
0 & 0 \\
0 & 0 \\
0 & 0 \\
0 & 0 \\
0 & 0 \\
0 & 0 \\
0 & 0 \\
0 & 0 \\
0 & 0 \\
0 & 0 \\
0 & 1 \\
1 & 1 \\
1 & 1 \\
1 & 1 \\
1 & 1 \\
\hline
\end{tabular}

\section{NOUAL POINT COOROINATES}

$\begin{array}{rrrr} & & & \\ 0.000 & -30.000 & 0.000 & -0 \\ 27.250 & -30.000 & 0.000 & -0 \\ 27.250 & -30.000 & 17.250 & -0 \\ 0.000 & -30.0 .00 & 17.25 .0 & -0 \\ 0.000 & 18.625 & 17.250 & -0 \\ 0.000 & 18.625 & 0.625 & =0 \\ 0.000 & 18.625 & 0.000 & -0 \\ 8.625 & 18.625 & 0.000 & -0 \\ 18.625 & 18.625 & 0.000 & -0 \\ 27.250 & 18.625 & 0.010 & =0 \\ 27.250 & 18.625 & 0.625 & -0 \\ 27.250 & 18.625 & 17.250 & =0 \\ 18.625 & 18.625 & 17.250 & -0 \\ 8.625 & 18.625 & 17.25 .0 & =0 \\ 0.000 & -80.000 & 0.000 & -0 \\ 27.250 & -80.000 & 0.020 & -0 \\ 27.250 & -80.000 & 17.250 & -0 \\ 0.000 & -80.0 .00 & 17.250 & -0\end{array}$

$-0.000$

$-0.000$

$-11.000$

$=0.000$

$-0.000$

$-0.000$

$-0.000$

$-0.000$

$=0.000$

$-0.000$

$=0.000$

$-0.000$

$-=0.000$

$-0.000$

$=0.0 .00$

$-0.000$

$\because \because \cdots$ 


\section{TABLE S-2 LOW FFEOUENCY CCFFEE TAALE CASE A ITIME MISTOAY ANALYSIS)}

\section{IUMPEOA A S S D A TA}

NODE $\angle O A D$
UABER CASE

$X-A X I S$

NUMBER .... CASF. ............. FOYCE $Y-A X I S$
F.OFCE Z-AXIS 2-AXIS
FORCE $X-4 X I S$ YOHENI $Y-A \times I S$ MOMEHT

\section{I-AXIS}

MOHENT

Q $44200 E-01$

$.467 .00 E=01$

$=0$.

-44700E-01 $.44700 E-01 \quad .44700 E-01 \quad 0$.

$.44700 E-01 \quad .46700 \bar{E}-01 \quad 044700 \bar{E}-01 \quad-0$.

$432706=01$

- $432.701=0$.

$.4327 .0 E=01$

$.04400 E-02, .09400 E-02-0$

$.4377 .0 E-01 \longrightarrow 43270 E=0.1 \longrightarrow 4327.0 E=01=0$.

$.89400 \mathrm{E}-02 \quad .89400 \mathrm{E}-02 \quad .89400 \overline{\mathrm{C}}-02-0$.

$.89 .00 E=02 \quad 0.940 .0 E=02$

$\therefore 8.940 .0 E-102$

$+8.940 .0 E=02$

- $43270 E-01-0$.

$.43270 E-01$

.89LO0E=02

$.4327 D E-0$

- $43270 E-01$

$.89400 E=02$
$.89400 E-02 \quad .8940 .0 E=02$

$.89400 E-02$

-0 .

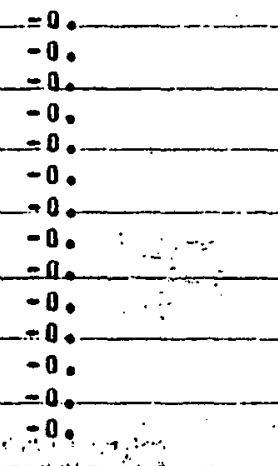

0.

.

-0 .

-0.
$=0$.
-0.

-0 .

-0.
$=0$.
-0.

-0 .

0 .

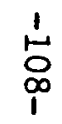

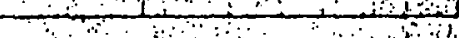

a d 
TAQLE 5-3 LOW EFEQUENCX_COEEEE TAQLE.CASE AUIHE.HIS.TORY ANALYSISI

NATURAL F FE Q UE NC IES

\begin{tabular}{|c|c|c|c|}
\hline $\begin{array}{l}\text { MOOF } \\
\text { NUTEER. }\end{array}$ & $\begin{array}{l}\text { CIRCULAF } \\
\text { FREQU:IICK } \\
\text { (PAO/SEC) }\end{array}$ & $\begin{array}{l}\text { EREQULACY } \\
\text { (CYCLES/S!EC) }\end{array}$ & $\begin{array}{l}\text { PEEICD } \\
\text { (SEC) }\end{array}$ \\
\hline 1 & $.547+[E+02$ & $.8712 E+01$ & $.114 B E+00$ \\
\hline 2 & $.25335+02$ & $.6005 E+01$ & $.1136 E+00$ \\
\hline 3 & $.11011 t+03$ & $.1751 E+02$ & $.5712 E-01$ \\
\hline 4 & $.2536[+10.3$ & $.4037 E+02$ & $.2477 E-01$ \\
\hline 5 & $.2615 \mathrm{E} \cdot 03$ & $.4163 E+02$ & .24028 \\
\hline
\end{tabular}


tahle 5-4a low fFequency coffee taple case a itime history)

M. O D E

MODE NUMUER .... 1

FREUUENCY $=0.71197$ HL

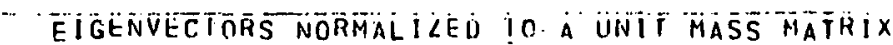

DISPLACEMENTSIKOIATIONSS ÖF UNRESTIRAINEL INOUẼ

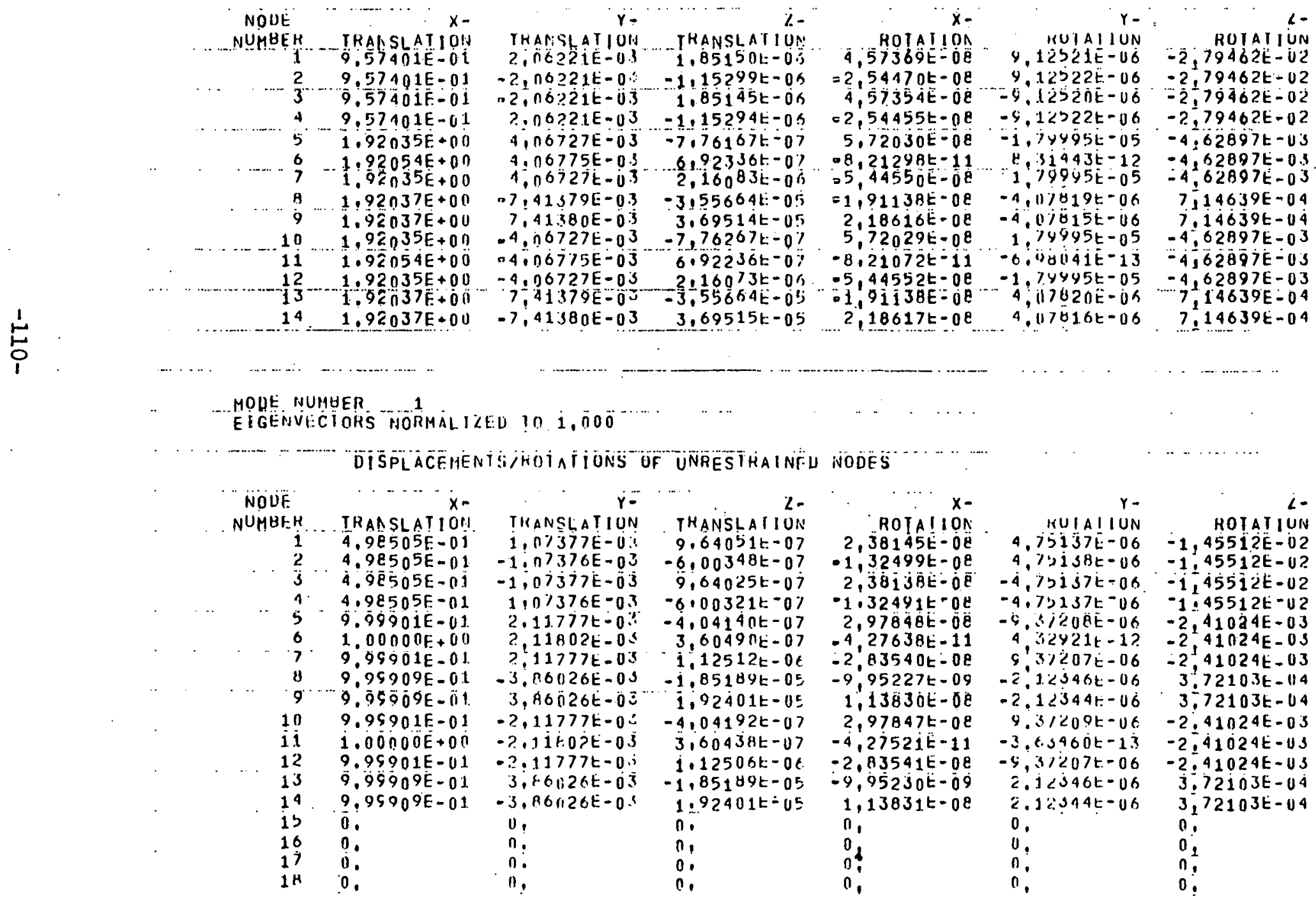


TABLE 5-4B LOW FFEQUENCY COFFFE TABLE (ASE A (TINE HISTOAY)

\section{MODE SITAPE}

MODE NUMHE

FRE DUENCY

B. $30 \% 224$

HL

EIGENVEC OORS NÖRMALIZ̈EU !

DISPLACËGENTS/KOIATIONS UF UNRESIRAINEU NOUES

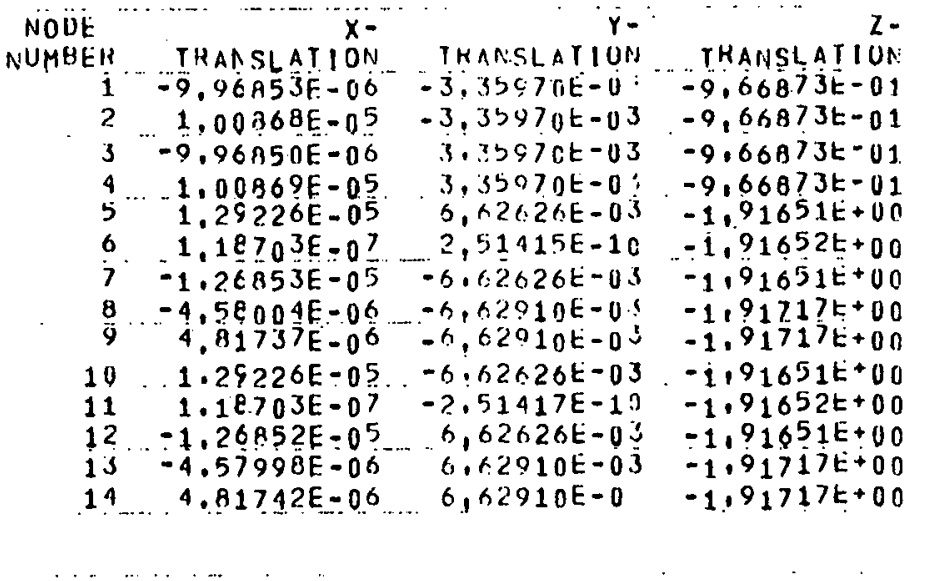

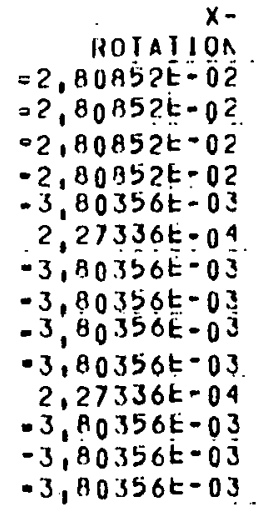

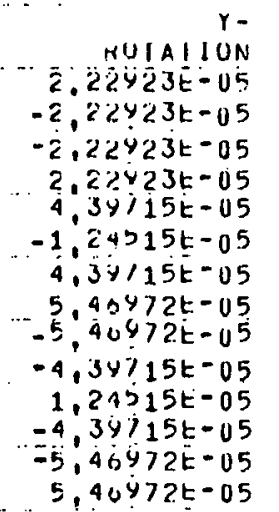

HOIAUIUN
$2,51739 E-U 7$
$-2,55193 E-07$
$2,5173 B E-07$
$2,55193 E-07$
$2,49156 E-07$
$-2,86103 E-10$
$-2,49728 E-07$
$-2,2482 U E-07$
$2,24917 E-07$
$2,49156 E-07$
$-2,86103 E-10$
$-2,49728 E-U 7$
$-2,24828 E-07$
$2,24917 E-07$

MODE NUMUER 2

EIGENVECIORS NORMALILED IO 1.000

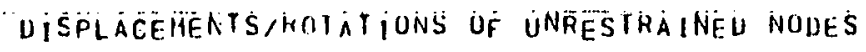

\begin{tabular}{|c|c|c|c|c|c|c|}
\hline NOUE & $x-$ & $Y-$ & $Z-$ & טרו & tul & ROIATIUN \\
\hline NUAHER & TRALSLAIION & TKANSLATIUN & TRANSLATIUN & $\begin{array}{r}\text { ROTA!ION } \\
46443 E-02\end{array}$ & $\begin{array}{r}H(1) A ! ! U N \\
1, \dot{B} 2>>E-U 5\end{array}$ & $\begin{array}{r}\text { ROIATIUN } \\
1,3130 B E-07\end{array}$ \\
\hline $\begin{array}{l}1 \\
2\end{array}$ & $\begin{array}{r}-5.19061 E-66 \\
5.26132 E-06\end{array}$ & $\begin{array}{l}-1,752.43 E-03 \\
-1,75243 E-0\end{array}$ & $\begin{array}{l}-5,04323 t-01 \\
-5,04323 t-01\end{array}$ & $\begin{array}{l}=1,46493 E-02 \\
=1,46493 E=02\end{array}$ & $\begin{array}{r}1,16277 t-05 \\
-1,10277 t-05\end{array}$ & $\begin{array}{r}1,31308 E-07 \\
-1,33109 E-0 ?\end{array}$ \\
\hline $\begin{array}{l}3 \\
4 \\
5\end{array}$ & $\begin{array}{r}-5.19959 E-06 \\
5.26134 E-06 \\
6.74 D 48 E-06\end{array}$ & $\begin{array}{l}1: 75343 E-03 \\
1: 75243 E-03 \\
3,45629 t-03\end{array}$ & $\begin{array}{l}-5,04323 E-01 \\
-5,04323 t-01 \\
-9,9965 \dot{E}-0 \dot{1}\end{array}$ & $\begin{array}{l}=1,46493 E-02 \\
-1,46493 t=02 \\
-1,98394 E-03\end{array}$ & $\begin{array}{r}-1.10277 t-05 \\
1,10277 t-05 \\
2,2 j \$ 56 t-05\end{array}$ & $\begin{array}{r}1: 31307 E-07 \\
-1: 33109 E-07 \\
1,29960 E-07\end{array}$ \\
\hline 6 & $6.15158 E-08$ & $1,31130 \mathrm{OE}-1 \mathrm{r}$ & $-9: 99602 k-01$ & 1,1857 QE 04 & $-t, 4 ; 975 t-06$ & $\begin{array}{l}-1: 4923 \text { PE- }-10 \\
-1: 30259 t-07\end{array}$ \\
\hline $\begin{array}{l}7 \\
B\end{array}$ & $\begin{array}{l}-6.61666 E-06 \\
-2.38696 E-06\end{array}$ & $\begin{array}{l}-3,15 \times 28 E-0.3 \\
-3,45776 E-0.5\end{array}$ & $\begin{array}{l}-9,99658 \mathrm{E}-0 j \\
-1,00000 \mathrm{t}+00\end{array}$ & $\begin{array}{l}-1,98394 \mathrm{t}-03 \\
-1,98394 t-03\end{array}$ & $\begin{array}{l}2,23557 t-05 \\
2,8302 t-05\end{array}$ & $-1,17271 \mathrm{E}-07$ \\
\hline$\dot{9}$ & $2.51275 E-00$ & $=3.45>70 t-03$ & $-1.00000 E+00$ & $=1,98394 \vec{E}-03$ & $-2,6>302 E-05$ & $1.17317 E-47$ \\
\hline $\begin{array}{l}10 \\
11\end{array}$ & $\begin{array}{l}6.74 \cap 45 E-06 \\
6.1515 B \dot{E}-0 H\end{array}$ & $\begin{array}{l}-3.15028 t-0.5 \\
-1.31140 t-10\end{array}$ & $\begin{array}{l}-9,99658 t-01 \\
-9,99662 k-01\end{array}$ & $\begin{array}{r}-1,98344 t-03 \\
1,18519 E-04\end{array}$ & $\begin{array}{r}-2,24356 t-05 \\
6,4 \times 476 t-06\end{array}$ & $\begin{array}{r}1.29960 E-07 \\
-1.492 J 2 t-10\end{array}$ \\
\hline 12 & $-6.61663 E-06$ & $3.456 .25 E-0.3$ & $-9,99658 t-01$ & $=1,98394 t-03$ & -2.2 Y S $60 \mathrm{E}-05$ & $-1.30259 E-07$ \\
\hline 13 & $-2.3 E A 93 E-06$ & 3. ヘ๖ 77 คะ -0.3 & $-1.00000 E+00$ & $-1.98394 t-03$ & $-2.83902 t-05$ & $-1.17271 E-U 7$ \\
\hline 14 & 2.5227 & $3,05776,-0.5$ & $-1,00000 t+00$ & $-1,98394 t-03$ & $2,40302 k-05$ & $1,17317 E-07$ \\
\hline 13 & n. & 0 . & $0:$ & O, & U, & $n$. \\
\hline 16 & 0 & 0 : & 0 : & 0, & 0 & 02 \\
\hline 17 & 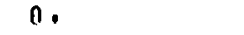 & $n$. & 0 . & n. & 0 , & 09 \\
\hline 14 & 0 , & 0! & 0. & 0, & 0 . & $0_{1}$ \\
\hline
\end{tabular}


TABLE 5-4C LOW FFEOUENCY COFFE TAPLE CASE A (TIME hISTOHY)

MODE S IF

MODE NUMUER 3

FREDUENCYY = $-17,5079.112$

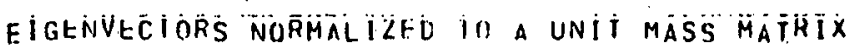

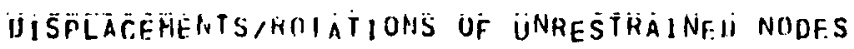

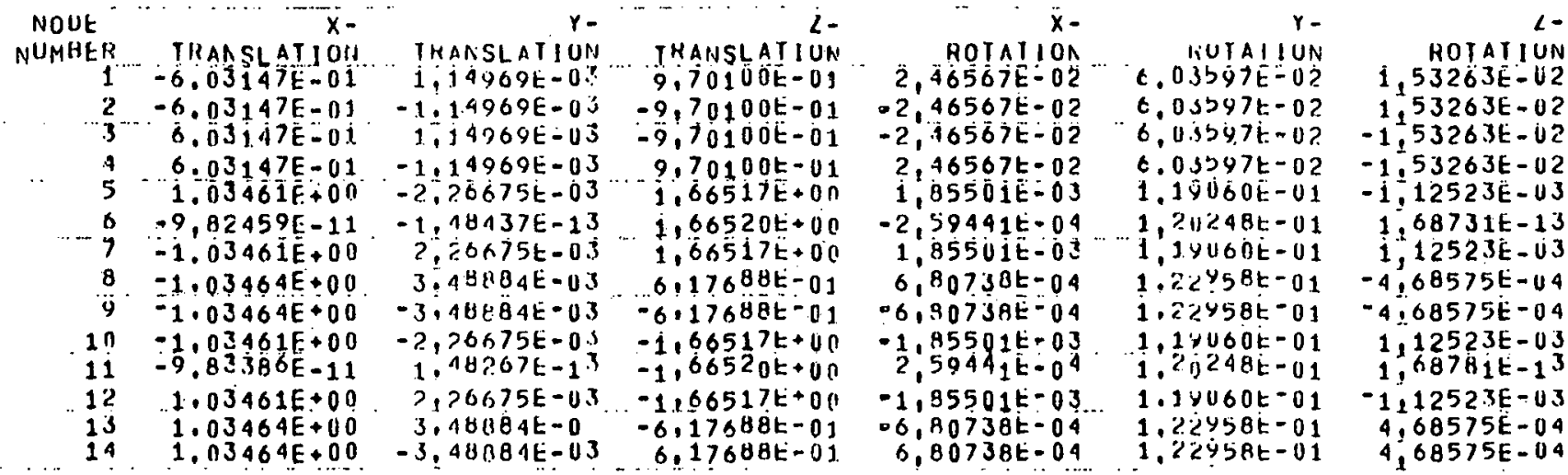

HODE NUMUEH 3

EIGETVECCIOF̈S NÖRMALIZZED IO 1,000

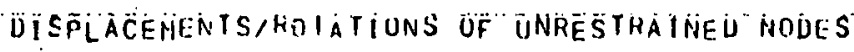

\begin{tabular}{|c|c|c|c|c|c|c|}
\hline | & $x-$ & $Y-$ & $i$ & $\bar{x}=$ & $Y$ & \\
\hline NUMBE. & TRAASLATION & TKANSLATIUN & THANSLATIUR: & ROIATION & MUIAIIUN & HOTATIL \\
\hline & $=207 E-01$ & $6.90422 \mathrm{E}-0$ & $1 t-01$ & 1,480 & $3,6 \%$ & $B \dot{E}-0 S$ \\
\hline 2 & $07 E-01$ & $-6,90422 \mathrm{E}-04$ & $E-01$ & $=1,48071 k-02$ & $3 t-02$ & $E-$ \\
\hline & 3.6250 & $6.90422 \mathrm{E}=04$ & $E-01$ & $071 \mathrm{E}=02$ & $3.62478 \mathrm{E}=0 ?$ & $E-1$ \\
\hline & $\begin{array}{l}3.62207 E=0.2 \\
6.21313 E=01\end{array}$ & $\begin{array}{l}-6,90422 t-04 \\
-1,36125 t-03\end{array}$ & $\begin{array}{l}4 t=01 \\
d t-01\end{array}$ & $\begin{array}{l}071 t=02 \\
399 E=03\end{array}$ & $\begin{array}{l}3.62 \\
7.17\end{array}$ & $\begin{array}{l}3 E-U^{3} \\
2 E-U^{4}\end{array}$ \\
\hline 6 & $=5.89996 \mathrm{E}-11$ & $-3,51405 t-14$ & $1,00000 t+00$ & $802 t-04$ & $7.22126 t-0 ?$ & $B E-1$ \\
\hline 7 & $=6.2 \mathrm{i} 3 \mathrm{~J} Z \mathrm{E}-0 \mathrm{i}$ & $j .36 .25 E-03$ & $t-01$ & $t=0 j$ & $i-42$ & -0 \\
\hline y & -6. & 03 & -01 & 4 & 7 & \\
\hline & $=6$. & & & & & \\
\hline 10 & $\begin{array}{r}-6.21313 \mathrm{E}-01 \\
-5.055 \mathrm{~F}-1\end{array}$ & $\begin{array}{r}-1.36125 \\
0.00 .280\end{array}$ & $-9,99$ & $=1,1$ & 7,14 & $\begin{array}{l}2 E-14 \\
A E-13\end{array}$ \\
\hline 11 & -5.9055 & $? .90$ & $t+0 n$ & 1 & $6 t=02$ & $E-1$ \\
\hline 12 & $6.21313 E-01$ & $1.36125 E-0.3$ & $-9,99984 t-01$. & $399 t-03$ & $38 t-02$ & $32 t-v$ \\
\hline 13 & 6.2133 & $2,04515 E-0.4$ & $-3.70940=-01$ & $3 E-04$ & $9 t-u ?$ & $3 E-U^{4}$ \\
\hline 14 & 0.2133 & $-? .0 .09$ & $3.70940 t-01$ & 4,0 & $2 t=02$ & $93 E-04$ \\
\hline 15 & 0. & :) & 0. & i, & $\dot{b}$ & $n$ \\
\hline & & & & & & \\
\hline 17 & 0 & $n$ & $\pi$ & 0 & " & $n$ \\
\hline 10 & 0. & $n \cdot$ & 01 & 0 . & 0 . & 0 \\
\hline
\end{tabular}


TABLE 5-40 LOW FFEQUENCY CCFFEe tAHLE CASE a (TIME histopy)

MO D $S$ It A P

MODE NUMUER

FREUUEA:CY $=40.3600$ HL

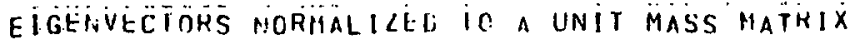

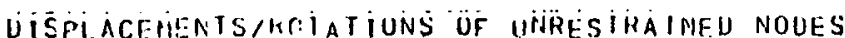

\begin{tabular}{|c|c|c|c|}
\hline 이: & $x-$ & $Y-$ & $L$ \\
\hline MEEH & THARSLATIOH & THANSLATION! & THANSLATIUN \\
\hline$i$ & $-2.36217 E+110$ & $-2.34097 E-0$ & $-4.13419 t-05$ \\
\hline ?. & $2.3 \in 2,4 E+110$ & $-? .34 \cap 97 t-04$ & $-4,13419 t-05$ \\
\hline 3 & $2 \cdot 36>47 E+100$ & $=2.34 \cap 97 E-04$ & $A+13419 k-05$ \\
\hline 4 & $\begin{array}{l}-2,36247 E+0 ! \\
-1.36755 E-63 \\
-1.37129 E-113\end{array}$ & $\begin{array}{l}-2.34097 t-09 \\
-1.0664 t-04 \\
-4.0308 E-04\end{array}$ & $\begin{array}{l}4,13419 t-05 \\
1.46837 t-08 \\
1.52100 t-14\end{array}$ \\
\hline , & $=1.36755 \bar{E}-0.3$ & $-A \cdot+0664 E-04$ & $-1.46836 \mathrm{E}-08$ \\
\hline & $\begin{array}{r}-5,0188 \mathrm{SE}=04 \\
5,01893 \mathrm{E}-04\end{array}$ & $\begin{array}{l}-2.40431 E-01 \\
-2,40431 t-01\end{array}$ & $\begin{array}{l}2.74993 t-06 \\
2.74993 t-06\end{array}$ \\
\hline $\begin{array}{l}10 \\
11 \\
12\end{array}$ & $\begin{array}{l}1.36755 E-03 \\
1.37129 E-03 \\
1.36755 E-03\end{array}$ & $\begin{array}{l}-4: 60664 E-04 \\
-4,61300 E-04 \\
-4,60664 E-04\end{array}$ & $\begin{array}{r}-1,46336 t-08 \\
-2,36265 t-15 \\
1,46836 t-08\end{array}$ \\
\hline $\begin{array}{l}13 \\
14\end{array}$ & $\begin{array}{r}5.01883 E-04 \\
-5\end{array}$ & $\begin{array}{l}-2.10431 t-01 \\
-2.40431 E-01\end{array}$ & $\begin{array}{l}-2.74993 t-06 \\
-2,74993 t-06\end{array}$ \\
\hline & 0 & & \\
\hline
\end{tabular}

\begin{tabular}{|c|c|}
\hline$x-$ & $\gamma-$ \\
\hline $\begin{array}{r}\quad R 014 ! 10 A \\
=1,13475 E=07 \\
=1,43475 t=07\end{array}$ & $\begin{array}{r}\text { RUIA!IUN } \\
-2.33 \% 29 t-07 \\
2.30729 t=07\end{array}$ \\
\hline $\begin{array}{r}1,43475 E-07 \\
1,43475 E-07 \\
-7,09631 E-07 \\
1,06656 t-16\end{array}$ & $\begin{array}{r}-2,36129 t-07 \\
2,3+129 t-07 \\
4,66448 t-07 \\
2,2016 \% t-15\end{array}$ \\
\hline $\begin{array}{l}7,09631 t=07 \\
7,09631 t=07 \\
7,09631 E=07\end{array}$ & $\begin{array}{r}-4.6044 \text { HE }-07 \\
-1,72519 t-07 \\
1.72519 \mathrm{E}-07\end{array}$ \\
\hline $\begin{array}{r}7,09631 t-07 \\
2,97748 t-16 \\
-7,09631 E-07 \\
-7,09631 \mathrm{E}-07 \\
-7,09631 \mathrm{t}-07\end{array}$ & $\begin{array}{r}4,60 Y 4 B t-07 \\
2,27177 t-15 \\
-4,66 Y 48 t-07 \\
-1.72919 t-07 \\
1.72519 t-07\end{array}$ \\
\hline
\end{tabular}

ROIAIIUN
$8.20167 E-U S$
$-9: 20167 E-U S$
$-8.20167 E-U S$
$920167 t-U 3$
$-4,05301 E-U 2$
$-4.05301 E-U 2$
$-4105301 E-U 2$
$-149751 E-U 2$
$199751 E-02$
$4.05301 E-U 2$
$4,05301 E-U 2$
$4.05301 E-U 2$
$1949751 E-02$
$-1,49751 E-U 2$

MOOE NUMUFR

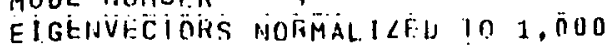

D ISPDLACETHENTS/HOTATIUNS UF IJNRESTHALINED NODES

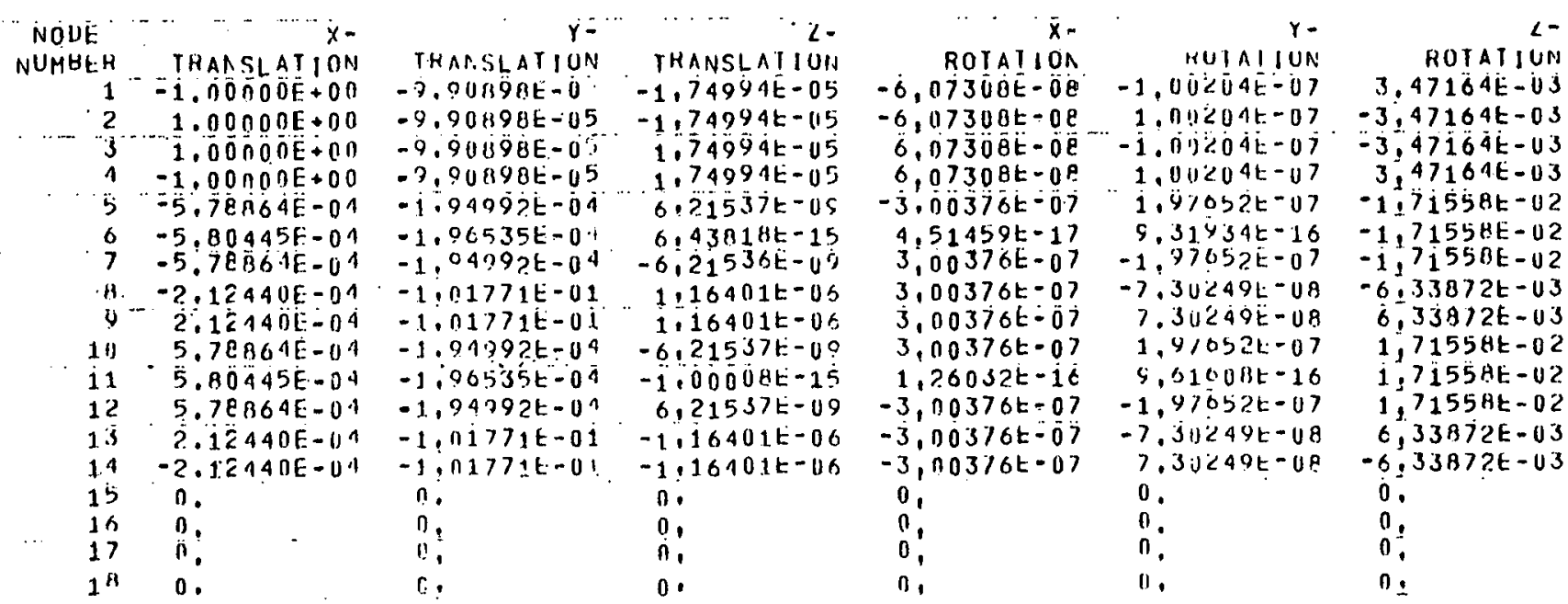


MODE NIJHIDF $S$

FREOUENCY $=41.6964 \quad 116$

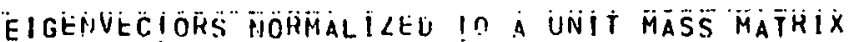

UISPLACFMI:NTS/AOINTIUNS UF UNHESTHAINEIN NODES

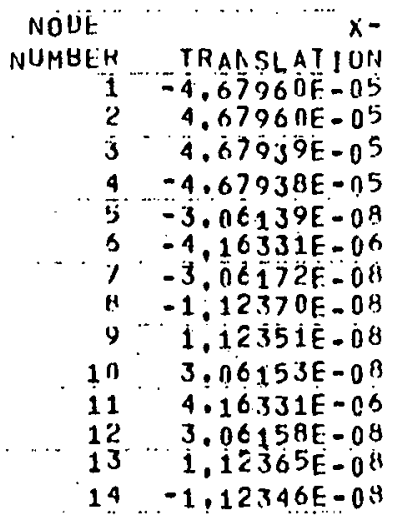

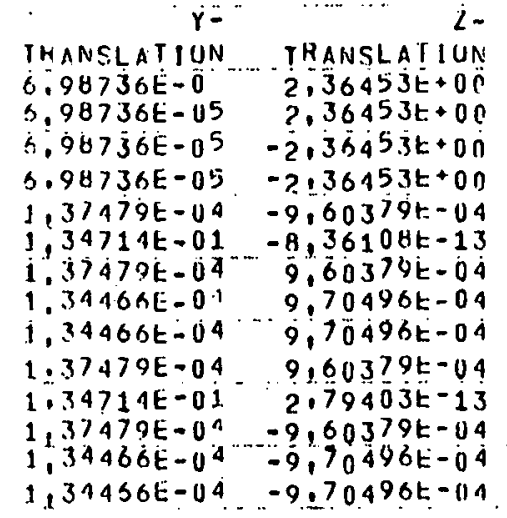

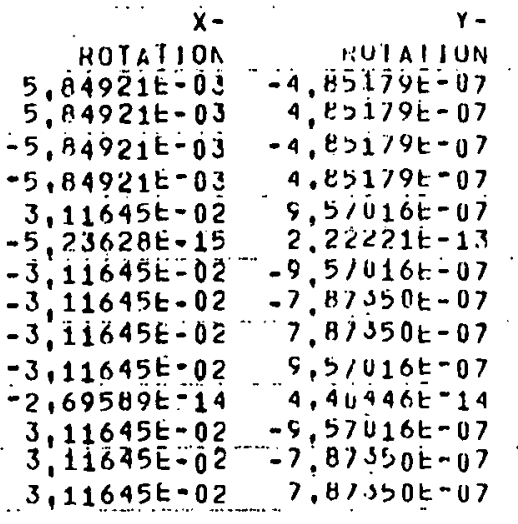

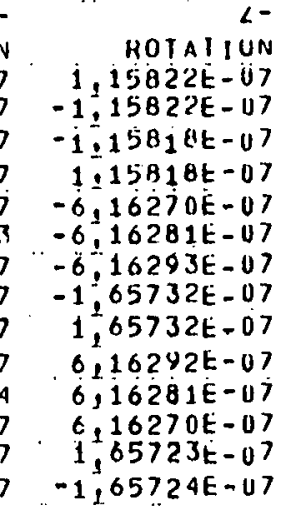

HODE NUMUER 5

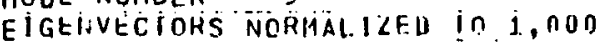

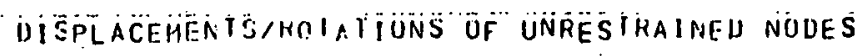

\begin{tabular}{|c|c|c|c|c|c|c|}
\hline $\begin{array}{l}\text { NOUE } \\
\text { NUMBt:? }\end{array}$ & IRANSLATION & TKANSLATIU:V & THANSLATIUN & ROTAIION- & 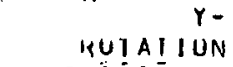 & ROTAIIUN \\
\hline & $-1,97008 E-05$ & 그 -.05 & $1,00000 t+00$ & $2,47373 E-03$ & $-2,0 \supset I \bar{Y} \cap 0 E-U 7$ & $29 t-08$ \\
\hline 2 & $1.97908 \mathrm{E}-05$ & ?.95507E-0S & $1,00000 t+00$ & $2,473736-03$ & $\begin{array}{r}2.03190 t-07 \\
-2.0 \div 190 t-07\end{array}$ & $\begin{array}{l}-4,89 B 29 E-U H \\
-4.89813 E-U B\end{array}$ \\
\hline $\begin{array}{l}3 \\
4 \\
5\end{array}$ & $\begin{array}{r}1.97899 \mathrm{E}-05 \\
-1.97999 \mathrm{E}-165 \\
-1.29471 \mathrm{E}-0 \mathrm{~B}\end{array}$ & $\begin{array}{l}2.05507 E-05 \\
2.95507 E-05 \\
5,81 A 22 E-U 5\end{array}$ & $\begin{array}{l}-1,00000 t+00 \\
-1,00000 t+0 n \\
-4,06160 t-04\end{array}$ & $\begin{array}{r}2,47373 E-03 \\
-2,473735-03 \\
1,31 \text { Bंण } 0=-02\end{array}$ & $\begin{array}{r}-2.02190 E-07 \\
2,00190 t-07 \\
4.01138 E-07\end{array}$ & $\begin{array}{r}4,89813 E-08 \\
4.89813 E-U \theta \\
-2,60530 t-07\end{array}$ \\
\hline $\begin{array}{l}6 \\
j \\
8\end{array}$ & $\begin{array}{l}-1.76073 E-00 \\
-1.25485 E-08 \\
-4.75232 E-09\end{array}$ & $\begin{array}{l}3.69729 E-02 \\
5.81422 E-05 \\
5.0 B 69 E-03\end{array}$ & $\begin{array}{r}-3: 53604 t-13 \\
4.06160 t-04 \\
4,10438 t-04\end{array}$ & $\begin{array}{l}=2,21451 t-15 \\
=1,31800 t=02 \\
=1,31800 t=02\end{array}$ & $\begin{array}{r}9,3 \times 807 t-14 \\
-4.04 \% 37 t-07 \\
-3.32983 t-07\end{array}$ & $\begin{array}{l}-2,60635 E-07 \\
-2,60640 E-07 \\
-7,00907 t-08\end{array}$ \\
\hline 1.0 & $\begin{array}{l}4.75150 E-09 \\
1.29477 F-0 n\end{array}$ & $\begin{array}{l}5.68679 E-05 \\
5.01122 E-05\end{array}$ & $\begin{array}{l}4,004 j 8 t-04 \\
4.06160 t-04\end{array}$ & $\begin{array}{l}-1, j i \text { juot }=02 \\
=1,31800 t-02\end{array}$ & $\begin{array}{l}3.32483 t-07 \\
4.04738 t-07\end{array}$ & $\begin{array}{l}7,00907 E-08 \\
2,60640 E-07\end{array}$ \\
\hline i i & $1.76 n 73 E-06$ & $5.69729 t-02$ & i.18164t-1? & $=1.14014 t-14$ & $1, t 627 ? k-14$ & $2.60635 E-47$ \\
\hline 12 & $1.2947^{\circ} \mathrm{E}-0 \mathrm{~B}$ & $5.8 .142 ? E-05$ & $-4,06160 t-04$ & $1,31800 t-02$ & $-4.04 / 385-07$ & $2.606 J 1 E-07$ \\
\hline $\begin{array}{l}13 \\
14\end{array}$ & $\begin{array}{r}4.7 E 21 \cap E-09 \\
-4.7 E-12 B E-09\end{array}$ & $\begin{array}{l}5.68679 E-05 \\
5.686791-0\end{array}$ & $\begin{array}{l}-4.10434 E-04 \\
-4.10438 E-04\end{array}$ & $\begin{array}{l}1.31800 t-02 \\
1,31800 t-02\end{array}$ & $\begin{array}{r}-3,3 \leq y+3 t-07 \\
3.32483 t-07\end{array}$ & $\begin{array}{r}7,00871 t-08 \\
-7.00872 E-0 E\end{array}$ \\
\hline 15 & in. & $n$. & i, & 0 , & 0 . & $0 ;$ \\
\hline 16 & 0 . & 0. & 0.1. & 0 ， & 0 : & 0 : \\
\hline 17 & $n$. & $n \cdot$ & $0 \cdot$ & 0. & 0. & n! \\
\hline $1^{3}$ & $0 . \ldots$ & $n$. & $0^{\circ}$ & 0. & 0 . & 0: \\
\hline
\end{tabular}




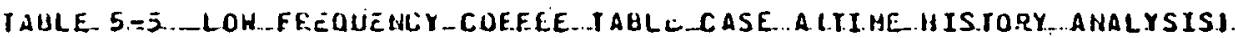

D I S PLACEMENT. HAXIMA

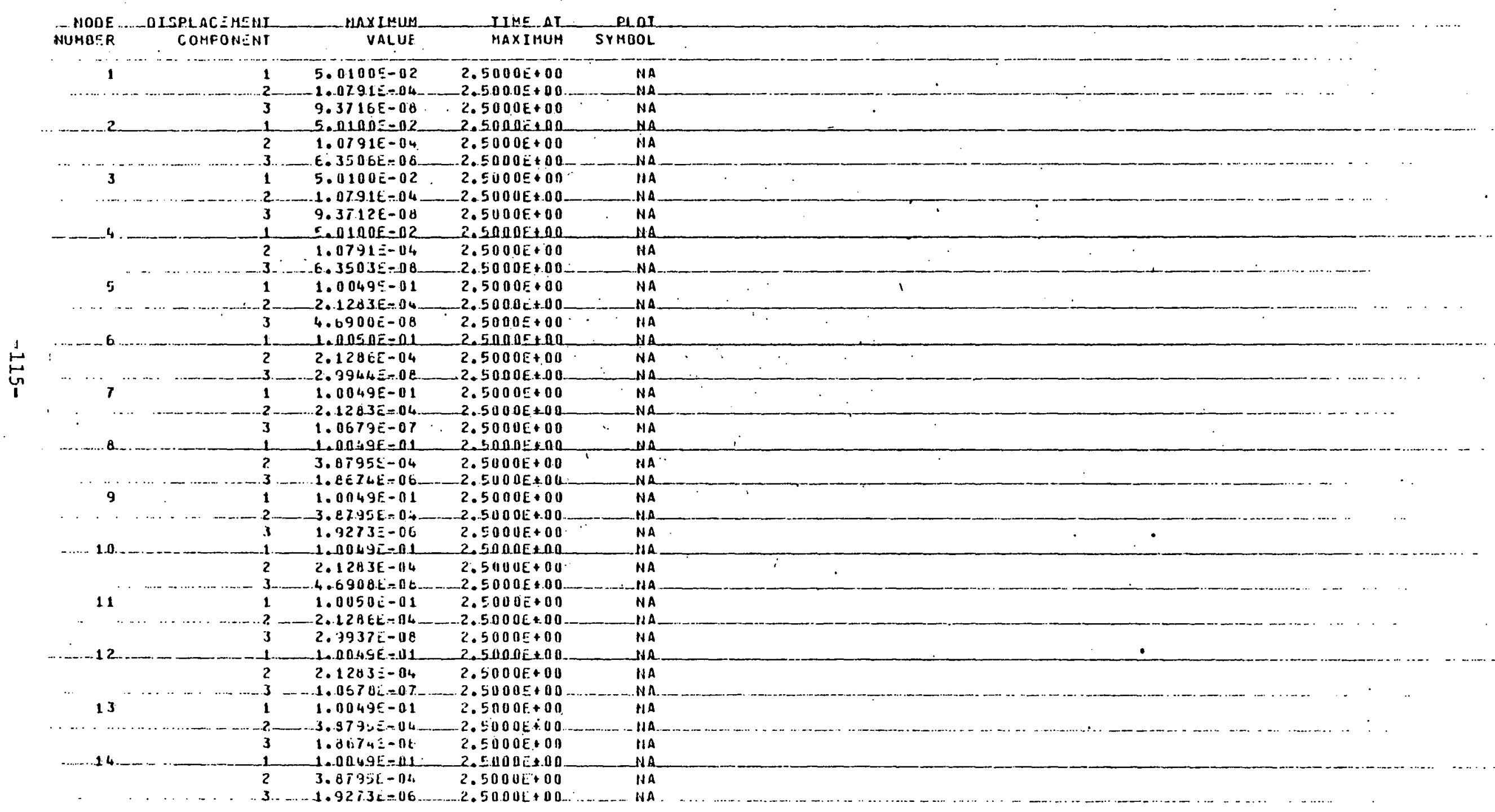




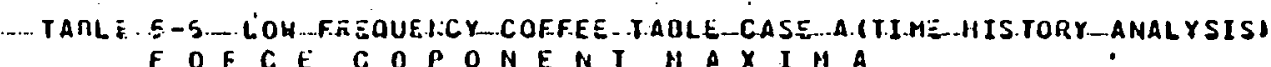

ELEMEITT TYPC NUHEEA $=2$

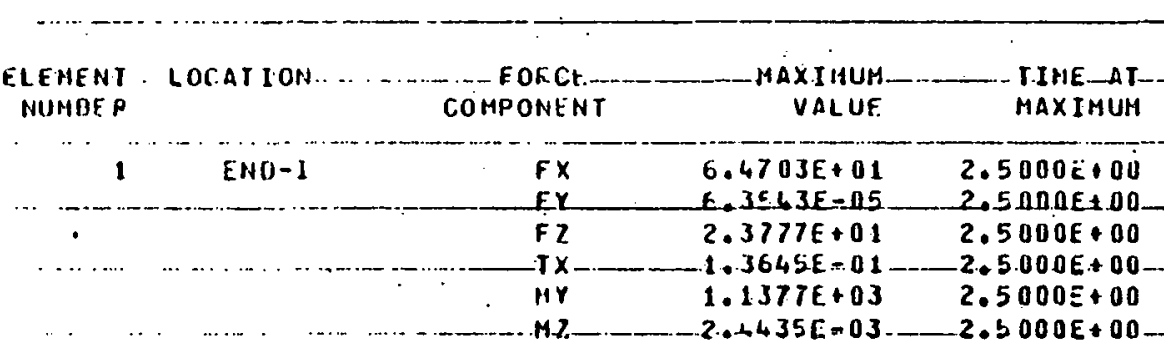

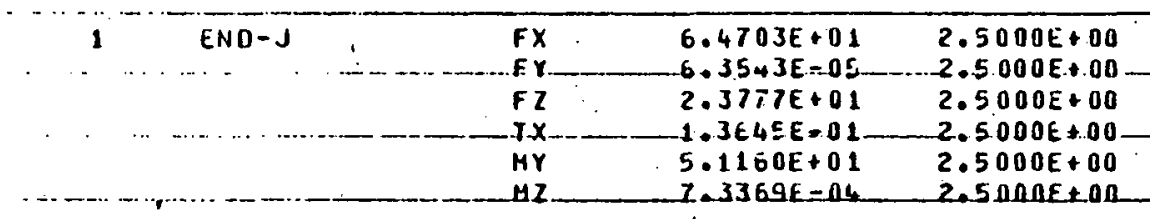

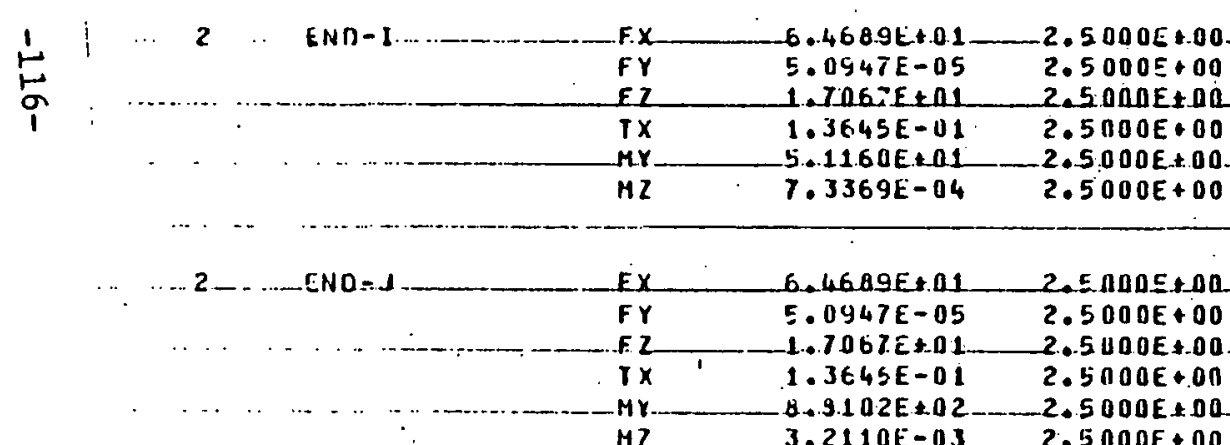

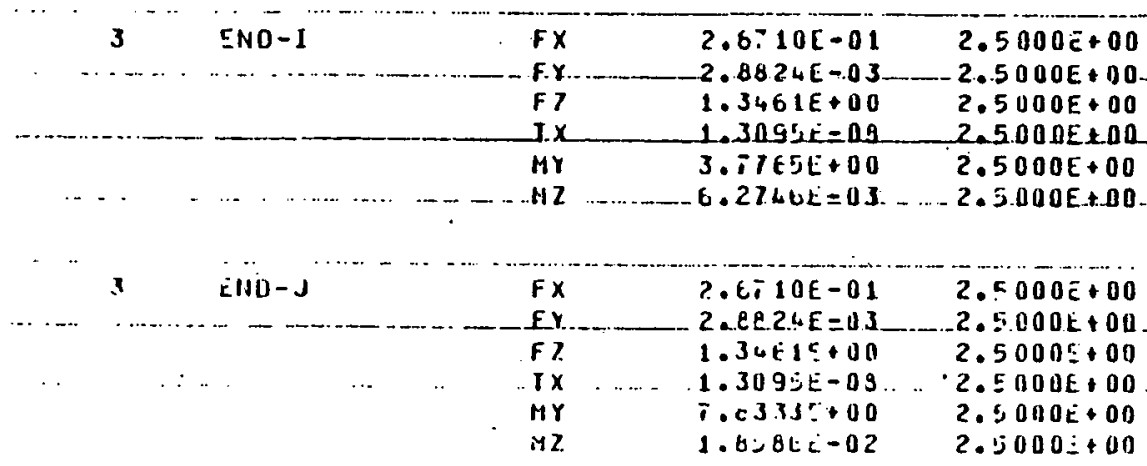




$\begin{array}{lr}F X & 2.6710 E-01 \\ F Y & 2.8195 E-03 \\ F 2 & 1.34 E 1 E 000 \\ I X & 1.3104 E-08 \\ M Y & 7.5333 E A 00 \\ 1: 2 & 1.858 E E-02\end{array}$

2. $.5 .000 E+.00$

$2.500 \cap E+00$

$2.5000 E+00$

2. $5000 E+00$

2. $5.000 E \pm 00$

$2.5000 E+00$

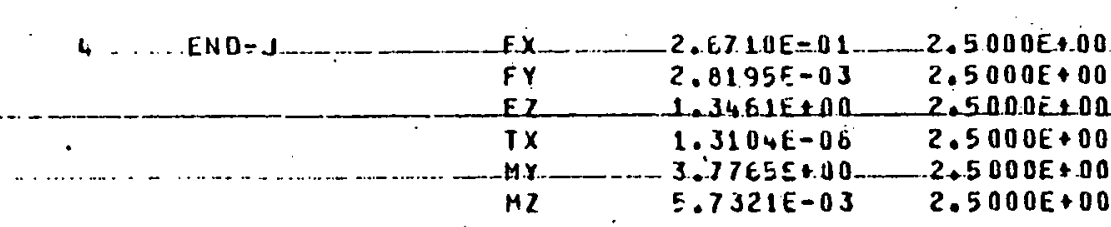

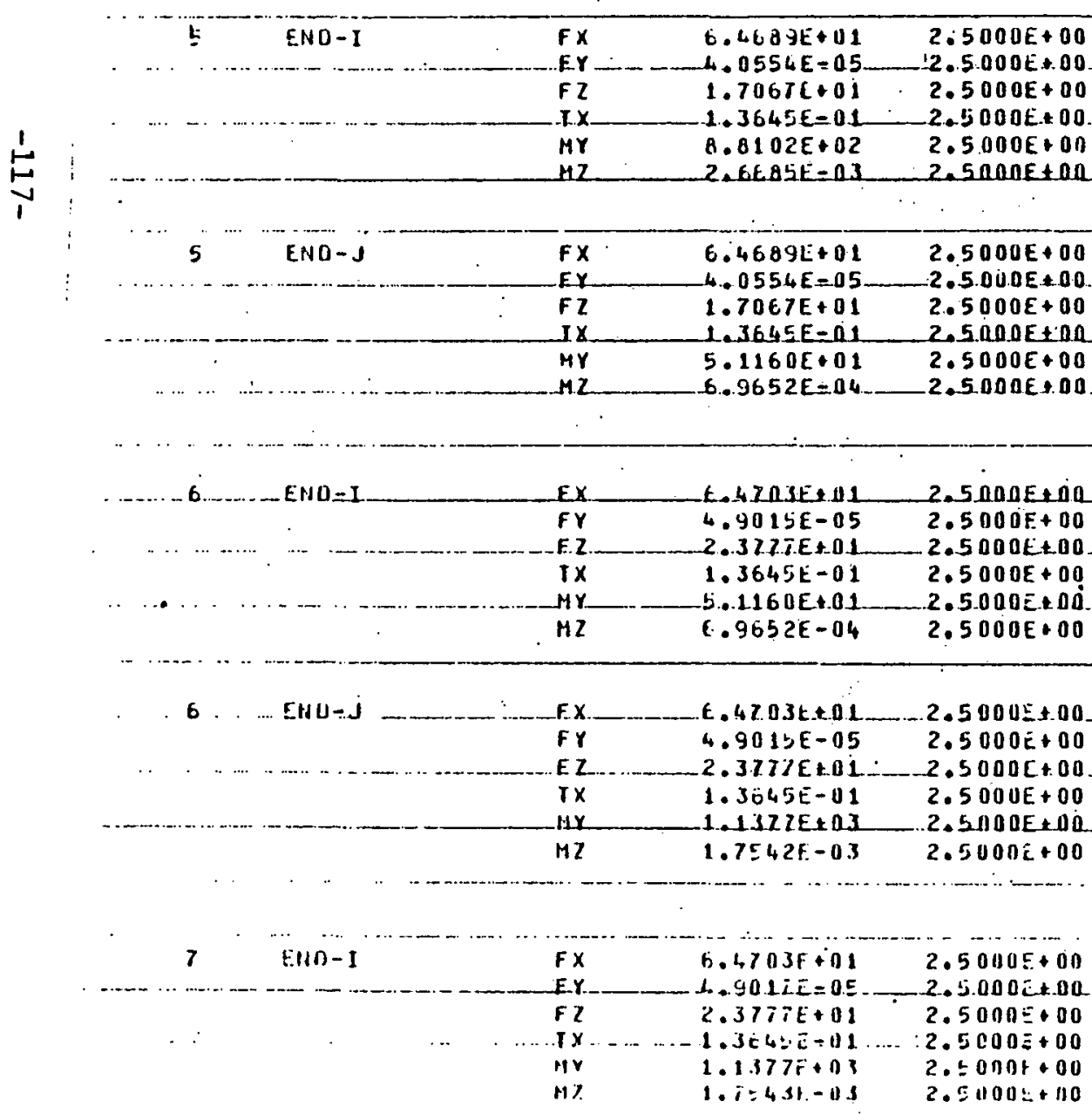




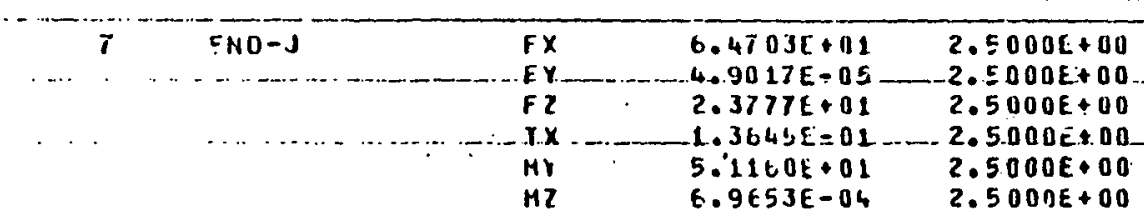

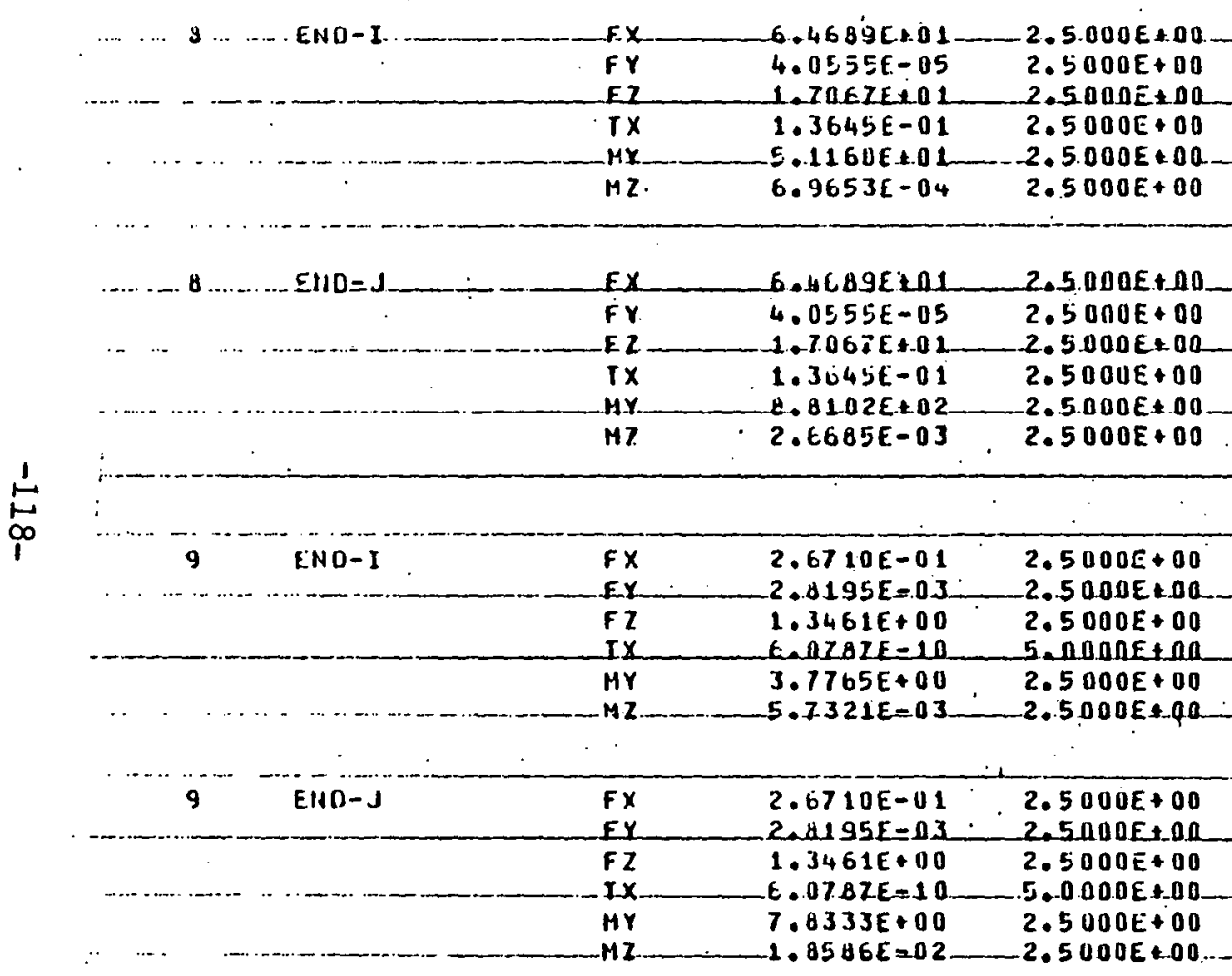

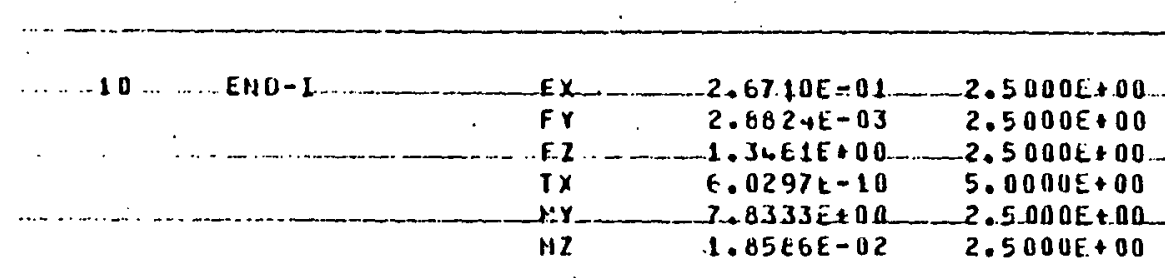

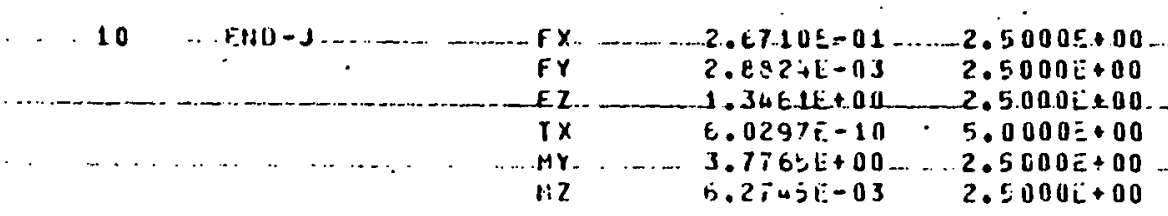




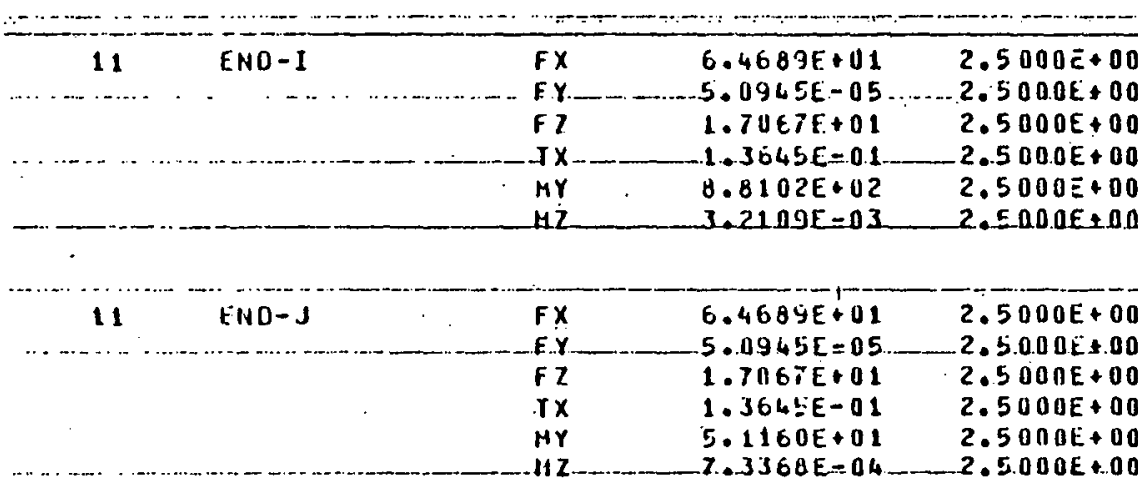

$\ldots+12 \quad E H a=1$

$\begin{array}{lrr}E X & E .4203 E \pm 01 & 2.5000 E+00 \\ F Y & 6.3561 E-05 & 2.5000 E+00 \\ E Z & 2.3727 E+0.1 & 2.5000 E+00 \\ T X & 1.3645 E-01 & 2.5000 E+00 \\ M Y & 5.1160 E+01 & 2.5000 E \pm 00 \\ H Z & 7.3368 E-04 & 2.5000 E+00\end{array}$

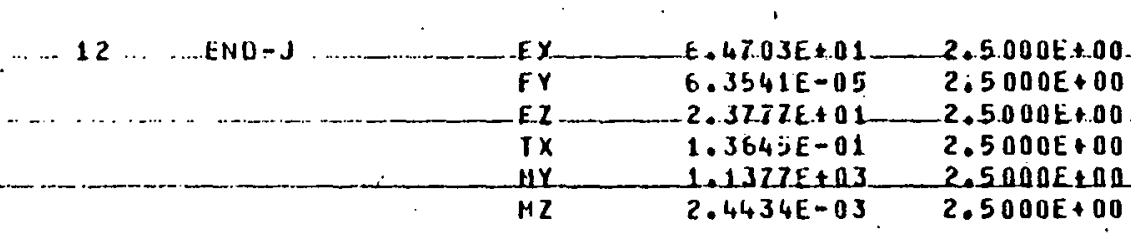

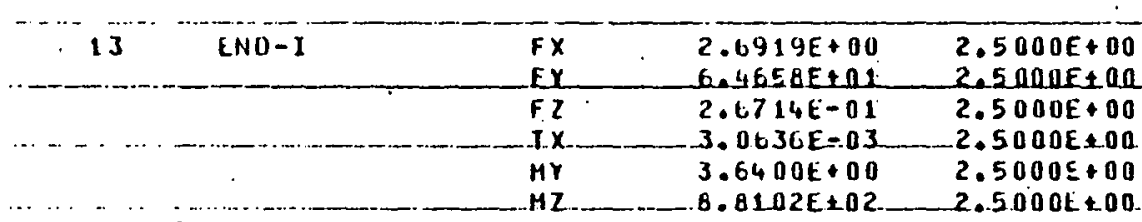

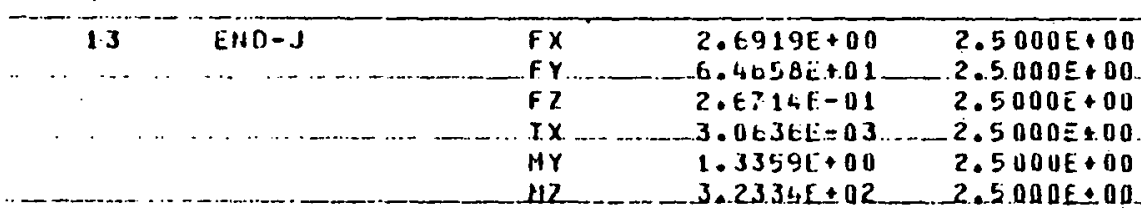

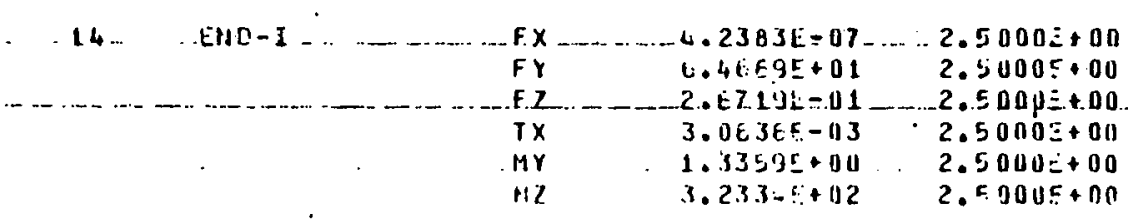


$14 \ldots \ldots$ - ENO - J

$\begin{array}{ccc}\text { EX } & 6.2383 E=02 & 2.5000 E+00 \\ F Y & 6.4669 E+01 & 2.5000 E+00 \\ F . Z & 2.67 .95=01 & 2.5 .000 E+00 \\ T X & 3.0636 E-03 & 2.500 .0 E+00 \\ -11 Y & -1.3360 E+00 & 2.5000 E+00 \\ M 7 & 3.2334 E+02 & 2.5000 E+00\end{array}$

15

END-1

$F X$

$3.2334 E+02$

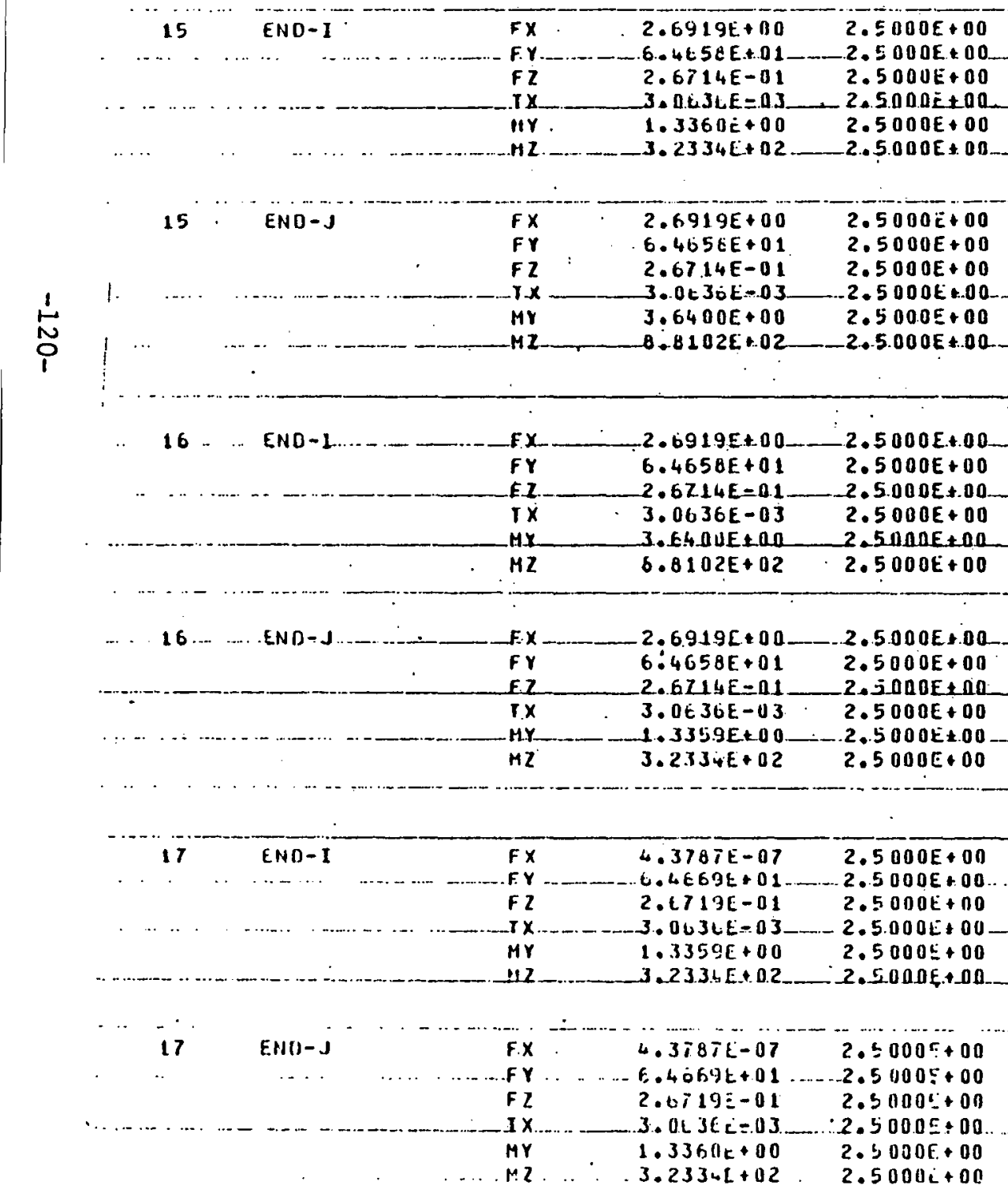




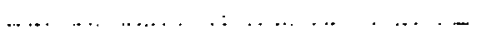

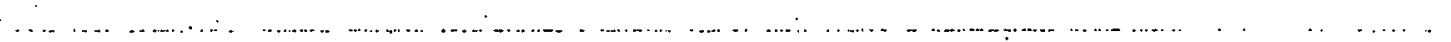

......18. - ....... ENO-I

F. $2.4 C 58 E+01 \quad 2.5000 E+00$

TX $\quad 3.0035 \mathrm{t}-03 \quad 2.500 .0 \mathrm{E}+00$

$\begin{array}{ll}14 Y & 1.3360 E+00-2.5000 E+00 \\ 4.2334 E+02,2.5000 E+00\end{array}$

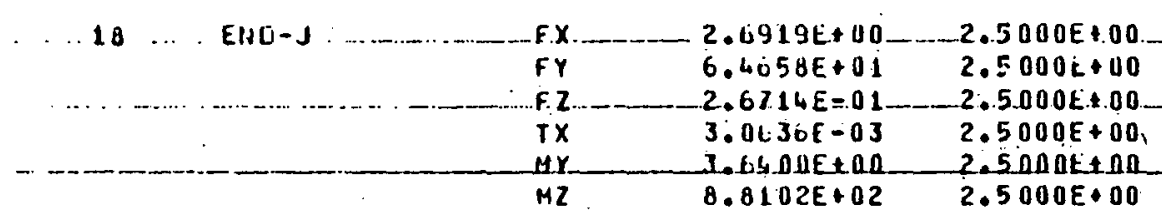

$\stackrel{1}{N}$

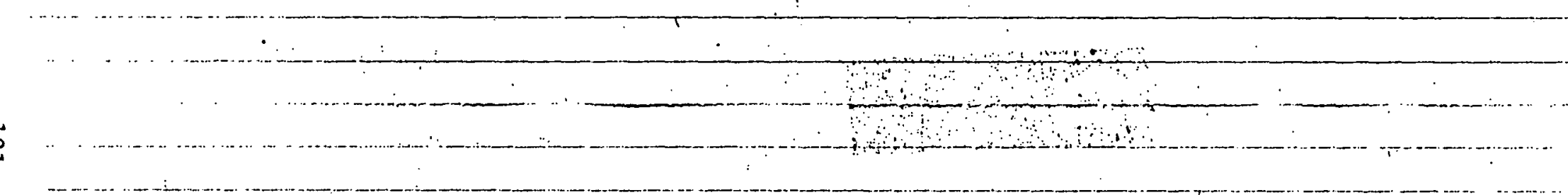

$\ldots+\ldots$

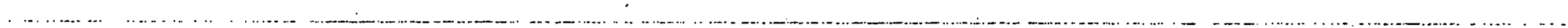

(n) 
TADLE_5=7__LH_EF_RUENCY_COEELE_TABLE_CASE_A.IJIME..H.ISTORY_ANALXSISI

$S$ I F E S S C D H P O NE H T MAX I MA

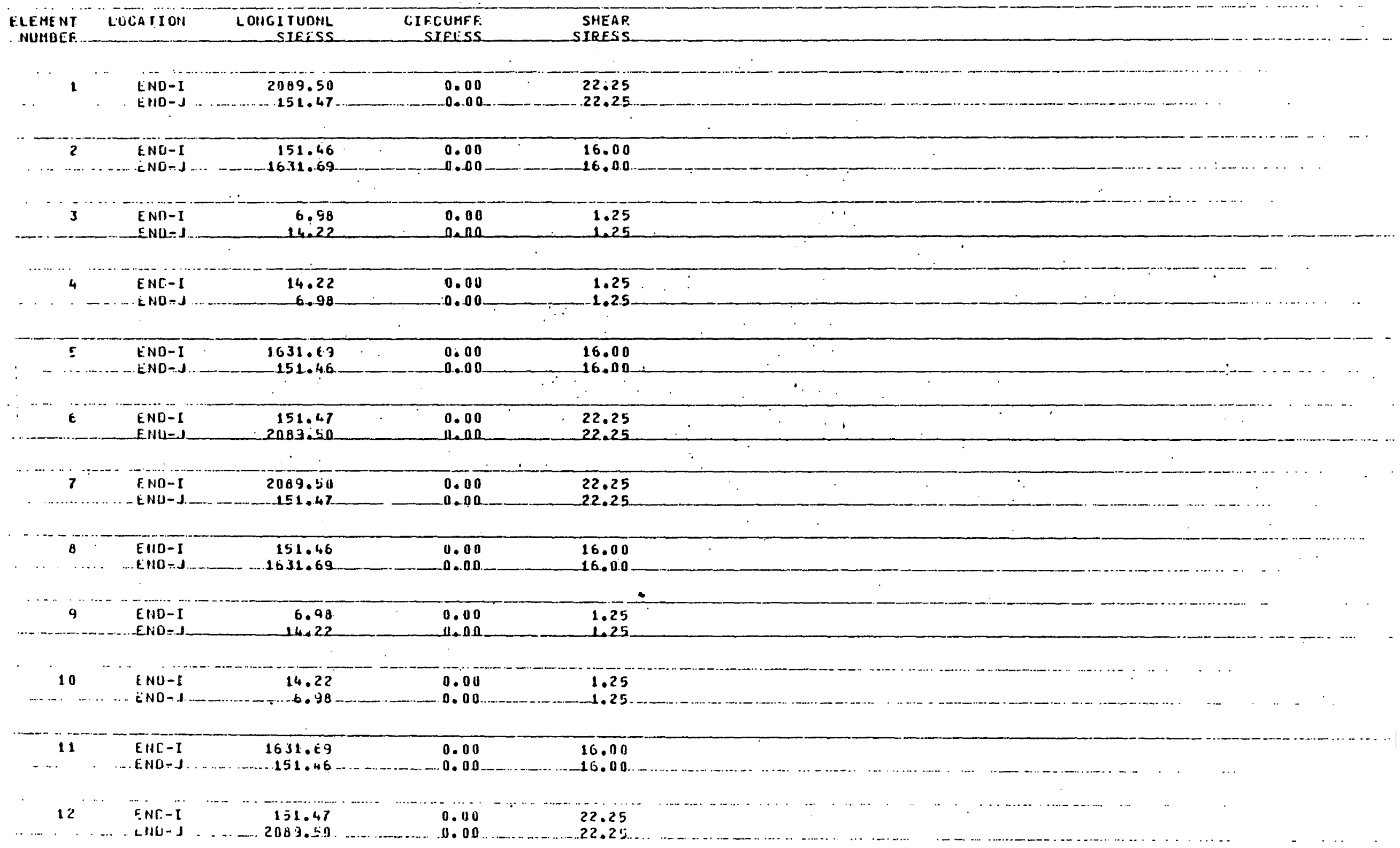




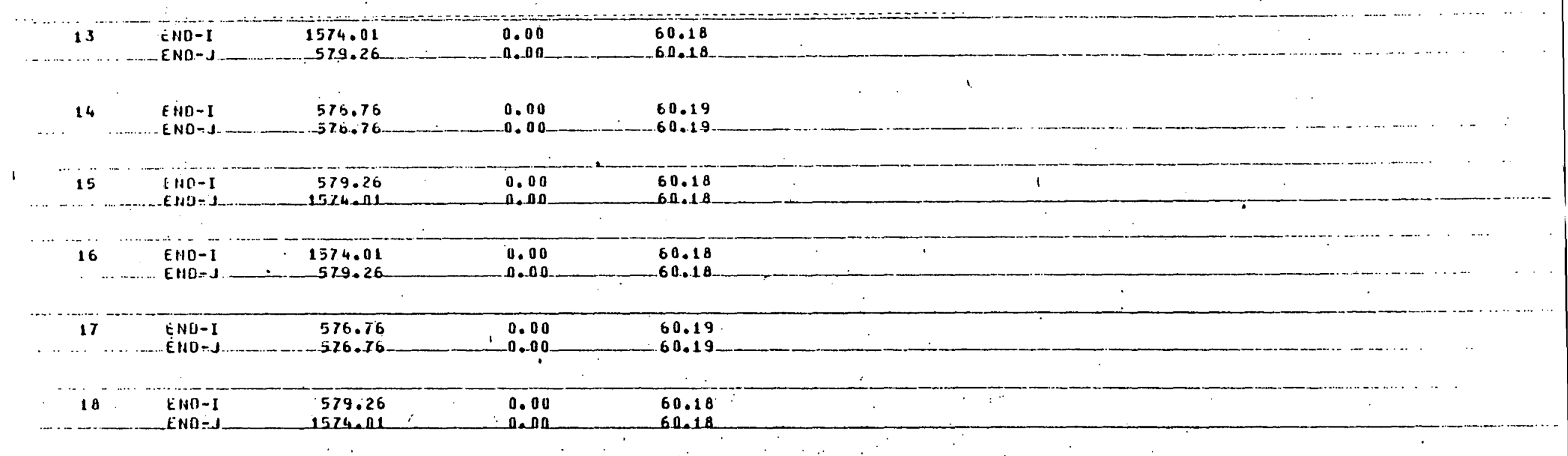




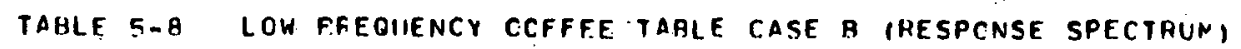
MCOAL PARTICIPATION FDCTIRS AND EARTHOUAKE RESPONSE

DIRECIION FÁTIOFS

$$
x=1.0006 \quad r=-0.0000 \quad z=-0.0000
$$

INDICATOR FCR DISPLACEMENT OF ACCEI.ERATION SPECTRIIM =

EQ.0 DISPLACENEAT

EO.I ACCELERAYION

MODAL PARTICIPATION F.PCTOFS

\begin{tabular}{|c|c|c|c|}
\hline MODE & X-DIFECTICN. & Y-DIAECIIOA & Z-OIDECTION \\
\hline 1 & . GORGE:ON & $.4 P 14 F-1 F$ & $.2194 E-06$ \\
\hline 2 & $.3749 E-07$ & $-.9541 F-17$ & $-.6074 E+0 n$ \\
\hline 3 & $-.3 ? 365-10$ & $.4532 F-16$ & - $\operatorname{R\cap Q7\cdot E-1j}$ \\
\hline 4 & $\therefore 1251 E-13$ & $\therefore$. 7 ? PF - ก? & $.7261 E-14$ \\
\hline 5 & $.2758 \mathrm{~F}-14$ & $.7450 E-0 ?$ & $.9110 E-13$ \\
\hline
\end{tabular}

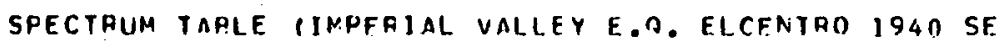

NLMPER CF PCINTS::
SCALE FACTOR :

INPUT SPECTALN

\begin{tabular}{|c|c|c|}
\hline & & \\
\hline POINT & PEFIOU & VALLEF \\
\hline 1 & I IG9AE-OR? & $.14505+03$ \\
\hline$?$ & - PA $6 \cap E-01$ & - $P A \cap \cap E+03$ \\
\hline 3 & $.580 \cap F-01$ & $.7750 E+03$ \\
\hline 4 & $.7100 E-01$ & $.7750 E: 03$ \\
\hline 5 & .9 IONE- - II & .440 EE ID 3 \\
\hline 6 & $.1140 E+00$ & $.11 A F E \rightarrow 04$ \\
\hline 7 & $.1410 E+00$ & . JIAPE ACA \\
\hline A & $.17 ? 0 E+00$ & $.7 n O O E .03$ \\
\hline 9 & . $\triangle$ NOOE $+O N$ & $.971 \cap E$ \& 03 \\
\hline 10 & $.250 \cap E, 00$ & $. A 7 I D E+O B$ \\
\hline 11 & . $\supset 30 E: 00$ & $.400 \cap E+03$ \\
\hline
\end{tabular}


GESPONSE FCR MCCE

FRF DUEACY $=$ P.71197 H

OISPLACFMENTS,FOTATIUNS OF UNRESIRAINED AOEES

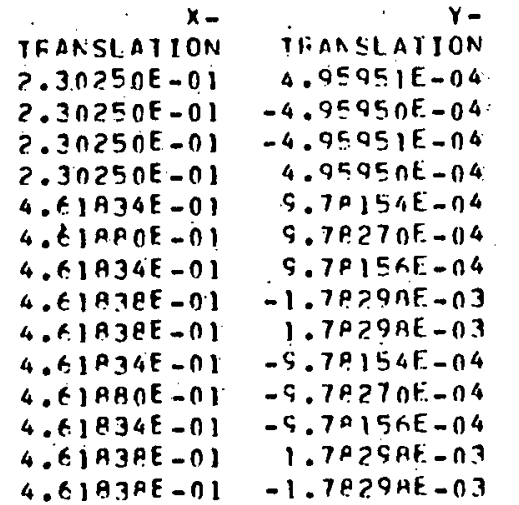

TRANSLATION

4.4527 GE- 07 $-2.77289 F-07$ $4.452645-07$ $-2.77276 F .07$ -1. An664E-07 1. 665 ก $3 E-07$ 5.196F.9E - OT $-8.55351 E-0 K$ B. AAG 6 IF-กG -1.466 ) AE-0? 1. $66479 E-07$ $5.19645 E-07$ $-8.55352 E-106$ 8. AnGG2E-DG

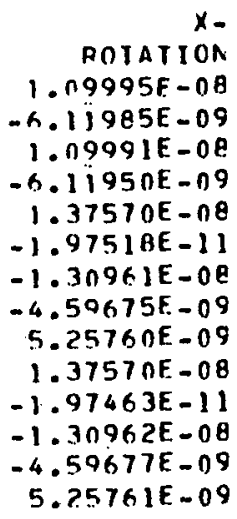

$x-$ PCTATION 2. $19454 E-06$ $2.19456 E-06$ $-2.19456 E-06$ $-2.1945 A E-06$ $-2.19456 E-06$ $-4.32 P 7 R E-06$
$1.99957 E-1$ ? $1.99957 E-1$ ?
$4.32877 E-O B$ $4.32 E 77 E-06$
$-9.807 R 3 E-07$ $-9.80783 E-07$ $-9.80773 F-07$ 4.3? T.7AF - 06 -1.07 P TE -13
-4.3 P P $7 E-06$ $9.80775 E-07$

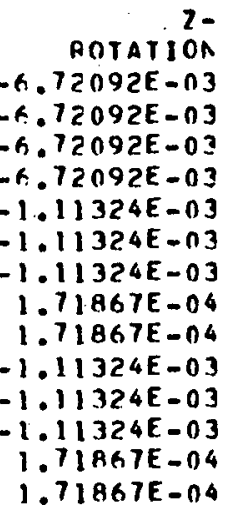

GESPONSE FCF MCCE

FREDUEACY = A.PCKZL $\vdash 7$

DISPLACFMENIS,FOIMTIONS OF UNRESTTÄIINED NODES

\begin{tabular}{|c|c|c|}
\hline NONF & & \\
\hline NUMREA & TFANSLATION & TFANSLATION \\
\hline 1 & $-1.4325 \times E-13$ & $-4 . A>816 E-11$ \\
\hline$?$ & $1.44956 E-1.3$ & -4.H—A 16E-11 \\
\hline 3 & $-1.43255 E-1.3$ & 4.A>P|RE- $\mid 1$ \\
\hline 4 & $1.44956 E-13$ & 4.A-B B RE - 11 \\
\hline 5 & $1.85705 E-13$ & $5.58247 E-11$ \\
\hline 6 & 1.705 QEE-15 & $=.61303 E-1 A$ \\
\hline 7 & - I..8???. & $-9.52247 E-11$ \\
\hline B & $-6.5 B 1$ APE -14 & $-5.5=655 F-11$ \\
\hline 9 & $6.92 \geq 94 E-14$ & $-5.5=655 E-11$ \\
\hline jo & $1.857 n P E-1.3$ & $-9.5 \geq 3475-11$ \\
\hline 11 & $1.70586 E-15$ & $-3.613 \cap A E-18$ \\
\hline 12 & $-1.8>297 E-13$ & $5.52247 E-11$ \\
\hline 13 & $-6.5 A \mid A O E-14$ & $5.5 \div 655 E-11$ \\
\hline 14 & $6.92302 E-14$ & $9.57+55 E-11$ \\
\hline
\end{tabular}

$7-$ IRANSLATION $-1.3 A 947 E-0 A$ $-1.38947 E-0 A$ $-1.3 A 947 E-0 B$ $-1.38947 F .0 B$ $-2.7541 \mathrm{AE}-0 \mathrm{~B}$ -2.7542 NE - OB $-2.7541 A F-08$ $-2.75513 E-0 B$ $755135-0 B$ 2.754 IAE 08 $-2.7541 B E-0 A$ $-2.75420 E-0 A$ $7541 B F-0 R$ $-2.75513 E-0 \mathrm{~A}$ $6.923 \cap 2 E-14$

ROTATIOA
$-4.03607 E-10$
$-4.03607 E-10$
$-4.03607 E-10$
$-4.03607 E-10$
$-5.46601 E-11$
$3.26700 E-12$
$-5.46601 E-11$
$-5.46601 E-11$
$-5.46 G O 1 E-11$
$-5.466 \cap 1 E-11$
$3.26700 E-12$
$-5.46 G O 1 E-11$
$-5.46 K O 1 E-11$
$-5.46 G O 1 E-11$

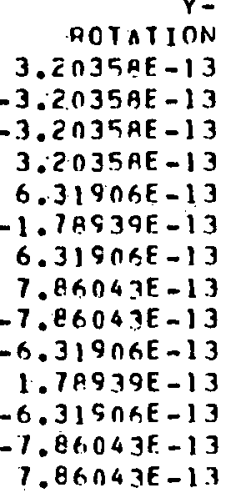

PO
POTATIOA
$3.61769 E-15$
$-3.66732 E-15$
$3.61768 E-15$
$-3.60733 E-15$
$3.52057 E-15$
$-4.11153 E-18$
$-3.58979 E-15$
$-3.23096 E-15$
$3.23223 E-15$
$3.58057 E-15$
$-4.11153 E-18$
$-3.58 A 79 E-15$
$-3.23096 E-15$
$3.23223 E-15$




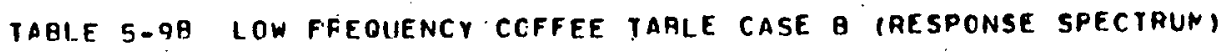

RESPONSE SPECTRUM OISPLACEMENT COMPONENTS

RESPONSE FCP MOCE 3
FREOUEACY $=17.5079^{3}+7$.

CISPLACEMEATS,FOTATIONS OF UNAESTRAINED AODES

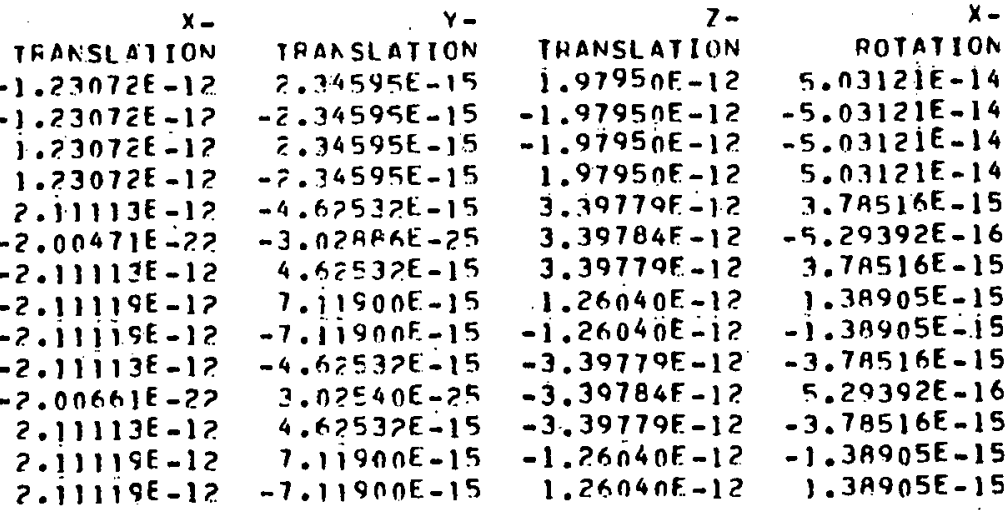

Rothing $1.23164 E-13$ $1.23164 E-13$
$1.23144 E-13$ $1.231 \mathrm{RE}-1.3$ $1.23164 \mathrm{E}-13$ $1.23144 \mathrm{E}-13$ $2.42942 \mathrm{~F}-1.3$ $2.45367 E-13$ $2.42542 E-13$ $2.5009 A E-13$ $2.5089 R E-13$ $2.42942 E-13$ $2.45367 E-1$ $2.42942 E-1.3$ $2.50896 E-13$ 2.5 OPQGE -13
Dotation $3.12733 \mathrm{E}-14$ $3.12733 E-14$ $-3.12733 E-14$ $-3.12733 E-14$ $-2.29603 E-15$ $3.44297 E-25$ .29603E-15 $-9.56130 E-16$ $-9.56130 E-16$ $2.29603 E-15$ $3.44399 E-25$ $-? .29603 E-15$ $9.56130 E-16$ $9.56130 E-16$

RESPONSE FCA MOCE

FRE OUEACY $=40.36 R A$ $+7$

CISPLACEMENIS, ERTATIONS OF UNAESTAAINER NODES

\begin{tabular}{|c|c|}
\hline $\begin{array}{l}\text { NODE } \\
\text { NUMAEA }\end{array}$ & IFANSLAIION \\
\hline 1 & $-1.71970 E-16$ \\
\hline$?$ & $1.71970 E-16$ \\
\hline 3 & $1.71970 E-16$ \\
\hline 4 & -1.7197 OE -16 \\
\hline $\mathbf{5}$ & $-9.9547 Z E-? 0$ \\
\hline h & $-9.9 R 192 E-20$ \\
\hline 7 & $-9.9547 ? E-20$ \\
\hline 8 & $-3.05333 E-20$ \\
\hline 9 & $3.553 .72 E-20$ \\
\hline 10 & $.9 .9547 \mathrm{CE}-20$ \\
\hline 11 & $9.9 R 192 E-20$ \\
\hline 12 & $.95472 f-20$ \\
\hline 13 & $3.6533 ? E-20$ \\
\hline 14 & $-3,65373 E-20$ \\
\hline
\end{tabular}

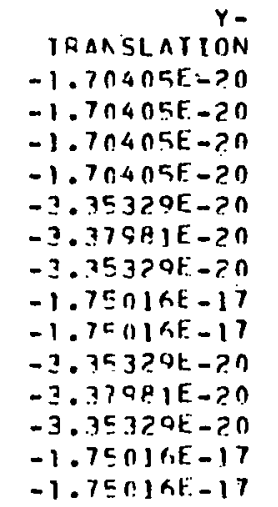

TRANSLATION -3. กतI 3 AE - 2. $-3 . \cap \cap 93 \cap E-2.1$ $3.0093 A F-2$ (.) $3.0 \cap 93 A E-2$ 1. OABAGE -2 1. In7 IBF. -3 -1. IGQAGE-?.4 2.00.174F-27 2. $01174 \mathrm{~F}-3$ ? $-1.068 B G F-24$ $-1.71983 F-31$ 1. ORBAGE - ? $-2.0 n T 74 E-32$ -2 . nก 174 F-? 2 1907a $x$ $-1.04439 E-23$ $-1.04439 E-23$ $1.04439 E-23$ $1.04439 E-23$ $-5.1655 B E-23$ $7.76375 E-33$ $5.16558 E-23$ 5. $1655 A E-23$ 5.1655BE-23 $5.1655 A F-23$ ?.1673AE - 32 ? 1673 EE $J 2$ $-5.16558 E-23$ $-5.16558 E-23$ $-5.16558 F-23$

.

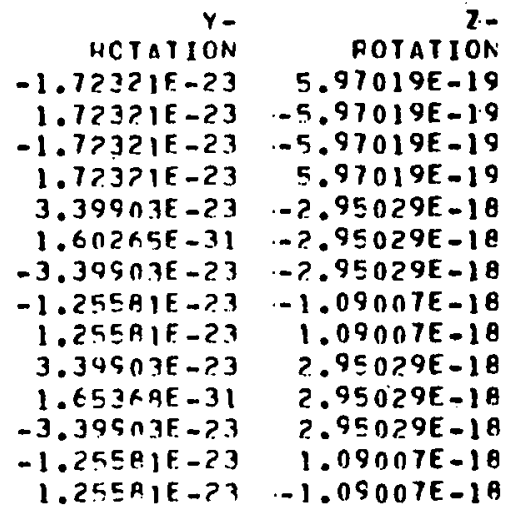


TABLE 5-9C LOW FFEQUENCY CCFFFe taple CASE a (RESPONSE SPECTRUN,

RESPONSE SPECTRUM CISPLACEMENT COMPONENTS ........................

RESPONSF. FCP MCEE

FRF DUEACY $=41 .+264$

$\rightarrow$

CISPLACFNFAIS, FOINYIONS OF̈ UNRFSIHAINEO NODES

\begin{tabular}{|c|c|c|c|c|c|c|}
\hline $\begin{array}{l}\text { NODE } \\
\text { NUMBF. }\end{array}$ & TFANSLATION & IFAASLATION & IRANSLATION & ROTATION & RCIAIION & ROTATION \\
\hline 1 & $-5.25 ? 04 E-2 ?$ & 7. . 421$\} E-? ?$ & $2.6537 \times 5-17$ & $6.56473 F-20$ & $5.4453 \cap E-24$ & $25990 E-24$ \\
\hline$?$ & $5.25204 E-2 ?$ & $11 E-? ?$ & $2.6537 \mathrm{AF}-17$ & $6.56473 E-20$ & & - \\
\hline 3 & IANE-?? & - AL & RF & -6.564 & 4 & \\
\hline 4 & $-5.251 P 0 E-? ?$ & 7.A4211E-?? & $A E-17$ & -6.56 & $5.4453 \cap E-24$ & -24 \\
\hline 5 & -3.435 AEE -25 & 1.54 & F. -20 & 3.49 & 1.074 & \\
\hline 6 & $E-23$ & 10 & - & $-5 . A 7$ & 94 & \\
\hline 7 & $6 E-2.5$ & 1.54 & $786 E-20$ & -3.40 & -1.074 & -1 \\
\hline A & $E E-25$ & $5 E-? 1$ & $19 ? 15-2 n$ & -3.49 & $-8.83+64 E-24$ & 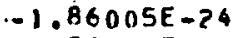 \\
\hline 9 & $94 F-25$ & $0915 E-21$ & $921 E-20$ & $-3.4 c$ & $4 E-24$ & -2 \\
\hline 10 & $3.436 \cap 4 E-25$ & -21 & $786 F-20$ & $E-19$ & $1.07409 E-23$ & .2 \\
\hline 11 & $7260 E-23$ & $4 E-1 A$ & $1 F=-30$ & -3.02 & $4.94324 E-31$ & -2 \\
\hline 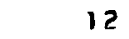 & $610 E-25$ & $4297 E-21$ & $86 E-20$ & $3.49767 E-19$ & $-1.07409 E-23$ & 65 \\
\hline 13 & $1.26110 E-25$ & $0915 f-? 1$ & $-1.0 R 9 ? 1 F-20$ & $3.49767 E-19$ & $-8.8 \geq \in 64 E-24$ & -2 \\
\hline 14 & -1.260 PEE - 25 & $1.50915 E-21$ & $08921 F .-20$ & $3.49767 E-19$ & $8.836 A 4 E-24$ & -7 \\
\hline
\end{tabular}

SOUAFE ROOT OF IHF SUM GF THE SOUARES OF THF NODAL DFFLECTIONS

CISPLACENENTSAFOTATIONS OF UNRESTRAINFD AODES

\begin{tabular}{|c|c|c|c|c|c|c|}
\hline $\begin{array}{l}\text { NODE } \\
\text { NUMAE.R }\end{array}$ & TRANSLATION & TFAASLATION & IRANSLATION & ROTATIOA & HCTATION & - \\
\hline 1 & $2.30250 E-01$ & $4.95951 E-n 4$ & $4.4549 .3 E-07$ & $1.10069 E-08$ & $2.1945 K E-n 6$ & $6.72092 E-03$ \\
\hline$?$ & $2.3 n \geq 5$ OF $-n 1$ & $\begin{array}{l}4.95950 E-04 \\
4.9505 E-04\end{array}$ & $\begin{array}{l}2.77637 F-07 \\
4.454 A \cap E-07\end{array}$ & 9 & $\begin{array}{l}2.19456 E-06 \\
2.19456 E-06\end{array}$ & $\begin{array}{l}6.72092 E-03 \\
6.72092 E-03\end{array}$ \\
\hline 4 & $\begin{array}{l}2.30250 E-11 \\
2.30250 E-01\end{array}$ & $4.9535 \cap E-04$ & $2.77624 \mathrm{E}-07$ & G. $13280 E=09$ & $2.1945 G E-06$ & $6.72092 E-03$ \\
\hline 5 & $4.61 A 34 E-01$ & S.7P.154E-04 & 1.AAGA5E -07 & $1.37571 E-08$ & $4.3 ? P 78 E-06$ & $1.11324 E-03$ \\
\hline 6 & 4.E\{AAOF-OI & S.7P.27.0E $=n 4$ & $1.6 A 766 E-07$ & 2. nñOLIE-11 & $2.02250 E-12$ & $11324 E-n 3$ \\
\hline 7 & $4.6 \mid A 34 E-01$ & S.7P15RE-04 & 5.20 .39 AF -07 & $1.30962 E-08$ & $4.3 ? E 77 E-0 B$ & $11324 E-03$ \\
\hline 9 & $1.61 A 38 E-01$ & $1.78 .29 \mathrm{AE}-0.3$ & $8.5535 \mathrm{KE}-0 \mathrm{~S}$ & AE -09 & $9.8 \cap 7 \times 3 E-07$ & hTE - n४ \\
\hline 9 & $4.6 \mid A 3 P E-01$ & $1.7 P 29 \mathrm{AE}-13$ & 8. ARG65F-06 & $5.25788 E-09$ & $9.8 \cap 773 E-07$ & $1.718 K 7 E-04$ \\
\hline in & $4.61834 E-01$ & $9.78154 E-04$ & 1.AR7 $\cap 9 F-07$ & $1.37571 E-08$ & $4.32 F 7 A E-O S$ & $1.11324 E-03$ \\
\hline il & 4.EJAEOE-OI & S.7P.27กE-04 & $1.68742 F-07$ & $2.0 \cap 14 \mathrm{AE}-11$ & 3.4650 GE - 1.3 & $1.11324 E-03$ \\
\hline$i_{2}$ & $4.6 \mid 934 E-01$ & $5.78156 E-04$ & $5.2 \cap 374 F-07$ & $1.30963 E-08$ & $4.32 P 77 E-06$ & $1.11324 E-03$ \\
\hline 13 & $4.61939 E-01$ & 1. 7 7029AE-0.3 & $5357 F-06$ & 09 & $9.807 A 5 F-07$ & .04 \\
\hline 14 & $4, E 1838 F-01$ & $1.7 P$ ج SAE - 03 & 8. ARGAGF-OG & 5.757896 .09 & $9.80775 E-07$ & $1.71 A 67 E-04$ \\
\hline
\end{tabular}


TABLE 5-90 LOW F.FEOUENCY CCFFEe TABLE CASE B (RFSPONSE SPECTRUN)

RESPONSE SPECTRUM FOACE COMPONENTS

SGUARE ROOT OF. ITE SUIM OF THF STUARES TF THE MODAL FORCES

(FOH ALL. FLFNENTS)

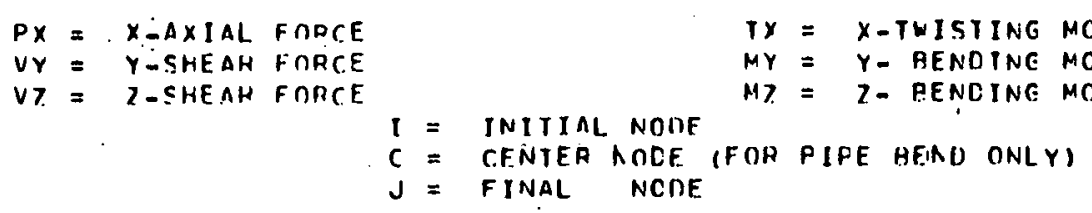




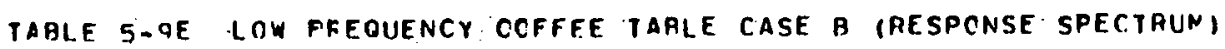

RESPONSE SPECTRUM FCRCE COMPONENTS

ELEMENT TYPF 13/D P I P $F$

PXI1) VY(I) VIII) $.2974 E+03 \quad .2184 E-03 \quad .1093 F+03$
1,1, ELEMENT NUIMAER 1

IXII) MY(I) MZ(I) PX( 6P7IF+00 .5229E+04 :.7737E-02 .29T4E+03
$\operatorname{Vr(J)}$ $.21 E 4 E-03$ v> ( $)$ $.1093 E \bullet 03$

$T \times(1)$
$.6271 E \div 00$
$.7183 E-02$

\section{1,1, ELEMFAT NIIMBEA 1 A)}

$\begin{array}{ccc}P \times(1) & V Y(1) & V Z(1) \\ .2973 E+03 & .1214 E-03 & .7 A 44 E+02\end{array}$

ELENEAT TYPF 13/C P'I PE

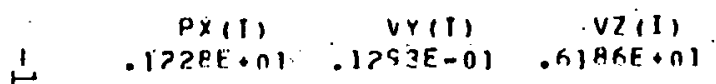

W

ELEMENT TYPF 13, O P I PE

PX(I) VY(I) VZIII

$\begin{array}{ccc}\text { VYII } & V Z(I) \\ .127 P F+01.1329 F-01 & .6196 E+01\end{array}$

ELEMENT TYPF (3/C F I $P E$

ELEMENT TYPF $13 /$ F F I PE

VY (II) VZIII

$.2974 \mathrm{~F}+0 ? .2991 \mathrm{~F}-03.1093 \mathrm{E}+03$

ELEMEA T TYPE $13 \% 0$ P I P F

$(x)(1)$

MY (I)

$M 2(I)$

$3183 \mathrm{E}-0$ ?

$\cos (d)$
$2973 . E+.03$

$V Y(J)$
$14 E-03$

$7844 E+0$

$(x \times 11)$

ELENENT NIJMBEA

9)

1,1, ELEMENT NIJMAER 1 101

$\begin{array}{cccc}T \times(1) & M Y(I) & M Z(I) & P X(J) \\ .1736 F-07 & .3600 F+0 ? & .8542 E-01 & .122 P E \div 01\end{array}$

VY(J)
$132 B E-01$

$V I(J)$
$6196 E+0)$

$\operatorname{tx}(\mathrm{T})$

$.1736 E-07$

$M Y(J)$
$.1736 E+02$

$M Z(J)$

IXI) MYII) MY (I) PXI)

$6271 \mathrm{~F}+00 \quad .4049 \mathrm{~F}+04 \quad .1502 \mathrm{E}-01.2973 \mathrm{E}+03$

$\operatorname{Vr}(J)$

$V 7(J)$

$1 \times(7)$

$.6271 E+00$

$\begin{array}{cc}M Y(J) & M Z(J) \\ .235] E+0.3 & .3390 E-12\end{array}$

1,1 FLEMENT NUMAFA 1 121

IXII) MY(I) MZ(I) PX(J)

$6271 F+00 \quad 2351 E+03 \quad 3390 E-02.2974 E+03$

. VYR (I)

V) (J)

$T \times(7)$ . ?95IE-03 . In93E+0.3 .6271E+00 .5229E+04 .1157E-01

$\operatorname{MY}(J)$

$M z(J)$ px(l) VYII

px(l) VYII

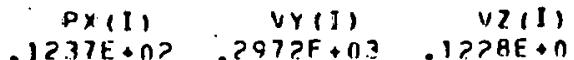

px(l) VYII
1,1, FLEMFAT NUMAER (1.3)

TX(I) MY (I) MZ(I) $P X(N)$

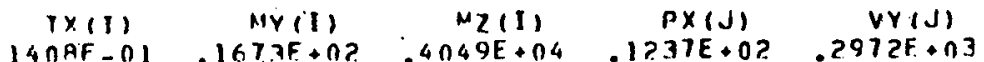

VT. (J) TX(J)

$.1228 E \mapsto 01$
1408E-01

$.6140 E+01$ 14RGE.0 
TABLE 5-9F LOW FFEOHENCY COFFE TAPLE CASE a (RESPONSE SPECTRUN)

RESPONSE SPECTRUM FCRCE COMPONENTS

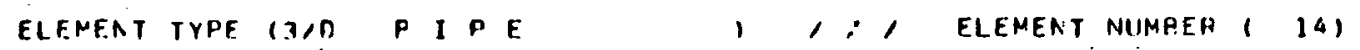




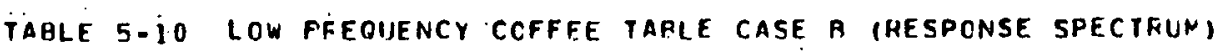

RFSPONSE SFECTRUM STRESS COMPONENTS

\begin{tabular}{|c|c|c|c|c|c|c|}
\hline $\begin{array}{l}\text { ELEMENT } \\
\text { NUMAEH }\end{array}$ & $\begin{array}{r}\text { ELFNENT } \\
\text { TYPE }\end{array}$ & $\begin{array}{l}\text { LCAD } \\
C A S E\end{array}$ & STATIOA & $\begin{array}{l}\text { I.NGTDI. } \\
\text { STAESS }\end{array}$ & $\begin{array}{r}\text { CIACUIMF } \\
\text { STRESS }\end{array}$ & $\begin{array}{r}\text { SHEAF } \\
\text { STRESS }\end{array}$ \\
\hline 1 & TARGENI & 1 & $\begin{array}{l}E \wedge C-1 \\
F \wedge C-J\end{array}$ & $\begin{array}{r}96 n 3.027 \\
696.134\end{array}$ & $\begin{array}{l}0.0 \text { ก0 } \\
0.000\end{array}$ & $\begin{array}{l}102.254 \\
102.254\end{array}$ \\
\hline 2 & TARGENT & $i$ & $\begin{array}{l}E \wedge C-I \\
E \wedge[-J\end{array}$ & $\begin{array}{r}696.073 \\
7499.011\end{array}$ & $\begin{array}{l}0.0070 \\
0.000\end{array}$ & $\begin{array}{l}73.554 \\
73.554\end{array}$ \\
\hline 3 & TANGENT & 1 & $\begin{array}{l}\text { EAL }-1 \\
\text { EAC }=U\end{array}$ & $\begin{array}{l}32.101 \\
4.5 .357\end{array}$ & $\begin{array}{l}0.000 \\
0.000\end{array}$ & $\begin{array}{l}5.757 \\
5.757\end{array}$ \\
\hline 4 & TARGENT & 1 & $\begin{array}{l}E A[-I \\
\operatorname{En}[-]\end{array}$ & $\begin{array}{l}+5.357 \\
.22 .101\end{array}$ & $\begin{array}{l}0.0 n \pi \\
0.000\end{array}$ & $\begin{array}{l}5.757 \\
5.757\end{array}$ \\
\hline 5 & TANGENT & 1 & $\begin{array}{l}E \cap C_{-}-1 \\
E \cap C-J\end{array}$ & $\begin{array}{r}7499.001 \\
.996 .072\end{array}$ & $\begin{array}{l}0.000 \\
0.000\end{array}$ & $\begin{array}{l}73.554 \\
73.554\end{array}$ \\
\hline 6 & TAAGENT & 1 & $\begin{array}{l}\operatorname{Enc}-1 \\
\operatorname{EAC}-J\end{array}$ & $\begin{array}{r}696.134 \\
9603.027\end{array}$ & $\begin{array}{l}0.0 \mathrm{nn} \\
0.000\end{array}$ & $\begin{array}{l}102.254 \\
102.254\end{array}$ \\
\hline 7 & TAAGENT & 1 & $\begin{array}{l}\text { EAC-I } \\
\text { EAL-J }\end{array}$ & $\begin{array}{r}9603.027 \\
696.134\end{array}$ & $\begin{array}{l}0.0 n 0 \\
0.000\end{array}$ & $\begin{array}{l}102.254 \\
102.254\end{array}$ \\
\hline A. & TANGENT & i & $\begin{array}{l}\text { EAC }-1 \\
\text { EAL }-J\end{array}$ & $\begin{array}{r}696.072 \\
74.99 .001\end{array}$ & $\begin{array}{l}0.0 n 0 \\
0.0 n 0\end{array}$ & $\begin{array}{l}73.554 \\
73.554\end{array}$ \\
\hline 9 & TANGENT & $i$ & $\begin{array}{l}E A E-I \\
E \cap E=J\end{array}$ & $\begin{array}{l}.32 .101 \\
6.5 .357\end{array}$ & $\begin{array}{l}0.0 n 0 \\
0.0 n n\end{array}$ & $\begin{array}{l}5.757 \\
5.757\end{array}$ \\
\hline 10 & TANGENI & 1 & $\begin{array}{l}E A C-I \\
\text { EAD-J }\end{array}$ & $\begin{array}{l}45.357 \\
32.101\end{array}$ & $\begin{array}{l}0.00 n \\
0.00 n\end{array}$ & $\begin{array}{l}5.757 \\
5.757\end{array}$ \\
\hline 11 & IANGENT & 1 & 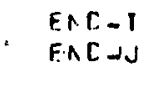 & $\begin{array}{r}7499.001 \\
696.073\end{array}$ & $\begin{array}{l}0 . \operatorname{nin} n \\
0 . \operatorname{con} 0\end{array}$ & $\begin{array}{l}73.554 \\
73.554\end{array}$ \\
\hline 12 & TANGENT & 1 & $\begin{array}{l}\text { EAC:-1 } \\
\text { EAC-J }\end{array}$ & $\begin{array}{r}696.134 \\
96 n 3.027\end{array}$ & $\begin{array}{l}0.0 n n \\
0.0 n n\end{array}$ & $\begin{array}{l}102.254 \\
102.254\end{array}$ \\
\hline 13 & TAAGENT & 1 & $\begin{array}{l}\text { EAL-I } \\
\text { EALE-I }\end{array}$ & $\begin{array}{l}723.7 .897 \\
26 \times 2.199\end{array}$ & $\begin{array}{l}0.000 \\
0.000\end{array}$ & $\begin{array}{l}276.563 \\
276.563\end{array}$ \\
\hline 14 & I $\triangle \wedge$ GENT & 1 & $\begin{array}{l}\text { EAC-I } \\
\text { EAC-J }\end{array}$ & $\begin{array}{l}2650.6 A 6 \\
2650.686\end{array}$ & $\begin{array}{l}n .000 \\
0.000\end{array}$ & $\begin{array}{l}276.608 \\
276.008\end{array}$ \\
\hline 15 & TAAGENT & 1 & $\begin{array}{l}\text { EAL-I } \\
\text { EALE-J }\end{array}$ & $\begin{array}{l}2652.199 \\
7733.697\end{array}$ & $\begin{array}{l}0.000 \\
0.000\end{array}$ & $\begin{array}{l}276.563 \\
276.563\end{array}$ \\
\hline 16 & TAAGENT & 1 & $\begin{array}{l}\operatorname{EAC}-I \\
\operatorname{EAC}-J\end{array}$ & $\begin{array}{l}77.73 .897 \\
\text { 2.6KE. } 199\end{array}$ & $\begin{array}{l}0.0 \cap 0 \\
n .00 n\end{array}$ & $\begin{array}{l}276.563 \\
276.563\end{array}$ \\
\hline 17 & TANGENT & 1 & $\begin{array}{l}\text { EAC }-1 \\
\text { FAC - I }\end{array}$ & $\begin{array}{l}\text { 2K5n.FAG } \\
\text { 2A5n. KAA. }\end{array}$ & $\begin{array}{l}0.0 \cap 0 \\
0.0 \cap 0\end{array}$ & $\begin{array}{l}276.608 \\
276.60 A\end{array}$ \\
\hline
\end{tabular}




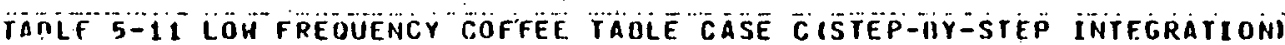

D 5 P A C MEM I MAX I M C

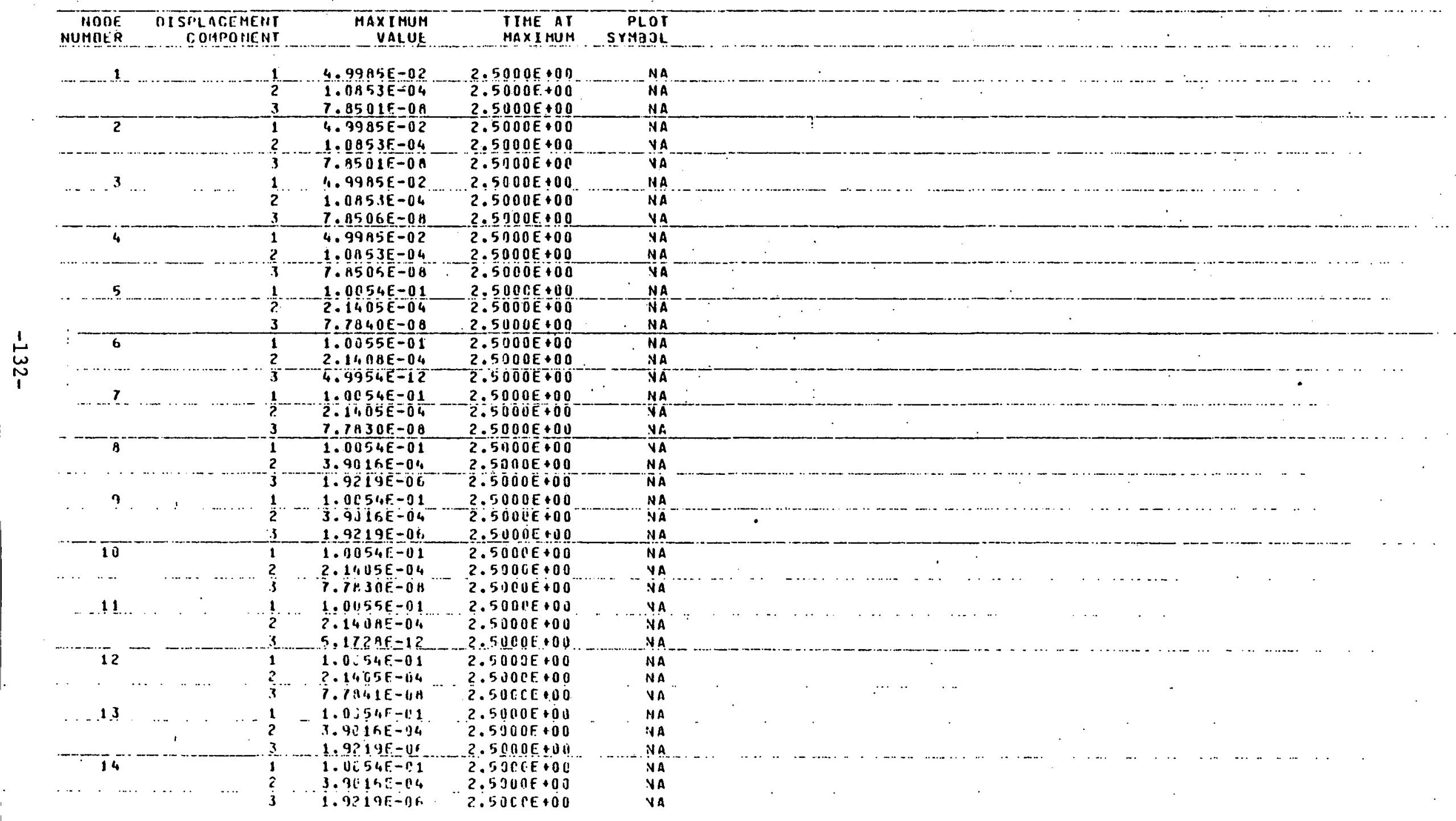




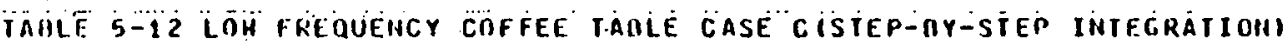

F O R C E C D M P O NE N I Y A X I MA

F.LEMENI IYFE HUHME: $=2$

ELEHETIT LOCAIIOAT
IIUMOER

$6.5074 t+0120.5000 E+00$

\begin{tabular}{|c|c|c|c|}
\hline $\operatorname{cHD}-1$ & $F x$ & $6.5074 E+01$ & $2.5000 E+00$ \\
\hline & $\begin{array}{l}F y \\
F Z\end{array}$ & $\begin{array}{l}5.5248 \overline{A E}-05 \\
2.3555 \mathrm{E}+01\end{array}$ & $\begin{array}{l}3.5000 E+00 \\
2.5000 E+00\end{array}$ \\
\hline & $\begin{array}{l}\text { II } \\
\text { MY }\end{array}$ & $\begin{array}{l}1.3822 E-01 \\
1.1323 E+03\end{array}$ & $\begin{array}{l}2.5000 E+00 \\
2.5000 E+00\end{array}$ \\
\hline
\end{tabular}

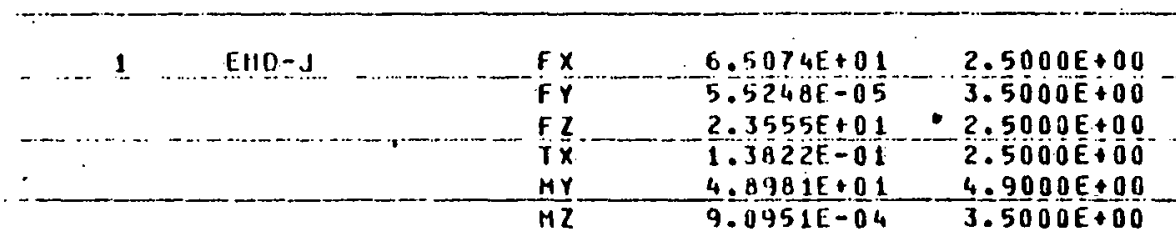

$\underset{\omega}{\omega}$

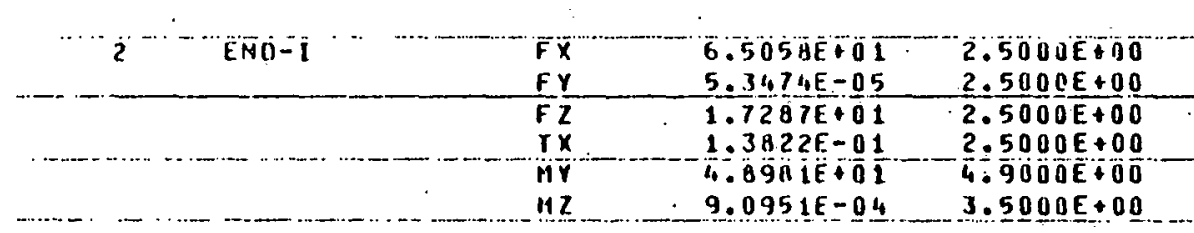

\begin{tabular}{|c|c|c|c|c|}
\hline 3 & END-J & $F \cdot \bar{y}$ & $\begin{array}{l}6.5058 E+01 \\
5.3471,6-05\end{array}$ & $\begin{array}{l}2.5000 E+00 \\
2.5000 E+00\end{array}$ \\
\hline & & $i$ & $1.7287 \mathrm{t}+01$ & $2.5000 E+00$ \\
\hline & -- & $\begin{array}{l}T x \\
i y y \\
112\end{array}$ & $\begin{array}{r}1.3+322 F-01 \\
8.0603 E+02 \\
3.2207 E-03\end{array}$ & $\begin{array}{l}2.5000 E+00 \\
2.5000 E+00 \\
2.5000 E+00\end{array}$ \\
\hline
\end{tabular}

\begin{tabular}{|c|c|c|c|c|}
\hline .. $\ldots . .3$ & END-I & $\begin{array}{l}F x \\
F y \\
F Y\end{array}$ & $\begin{array}{l}2.7055 E-01 \\
3.051 \text { HE }-03 \\
1.3634 E+00\end{array}$ & $\begin{array}{l}2.5000 E+00 \\
2.5000 E+00 \\
2.5100 E+00\end{array}$ \\
\hline 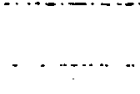 & & $\begin{array}{l}1 x \\
11 y \\
1: i\end{array}$ & $\begin{array}{l}8.80216-09 \\
3.1253 f+00 \\
6.1 .751 f-0.3\end{array}$ & $\begin{array}{l}2.5003 E+00 \\
2.5000 E+00 \\
2.500 U E+00\end{array}$ \\
\hline
\end{tabular}




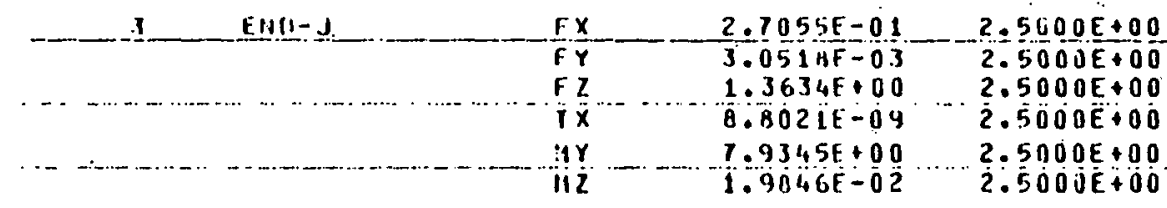

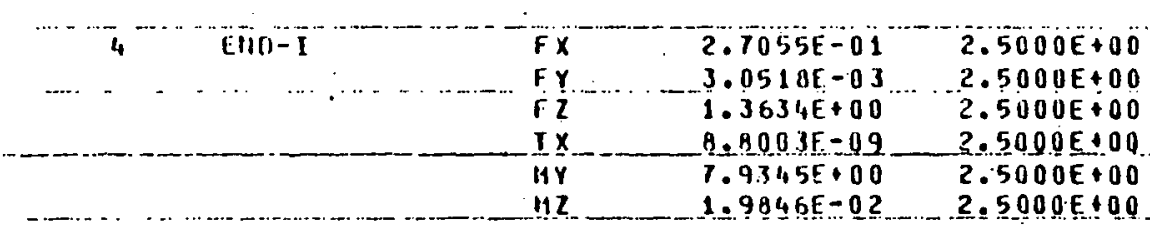

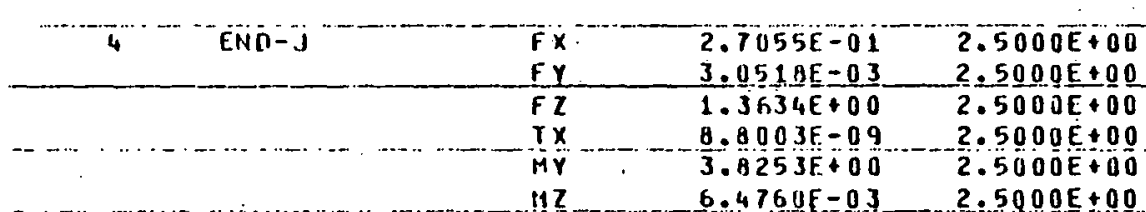

\begin{tabular}{|c|c|c|c|}
\hline 5 & $\frac{F X}{F y}$ & $\frac{5.5058 F+01}{5.3476 E=05}$ & $\begin{array}{r}2.5000 E+00 \\
2.5000 E+00\end{array}$ \\
\hline$\cdot .$. & $\frac{F}{T X}$ & 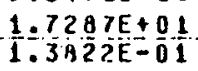 & $\frac{2}{2}=5000 E+00$ \\
\hline & MYY & $0.0603 \mathrm{~F} \cdot 02$ & $2.5000 E+00$ \\
\hline
\end{tabular}

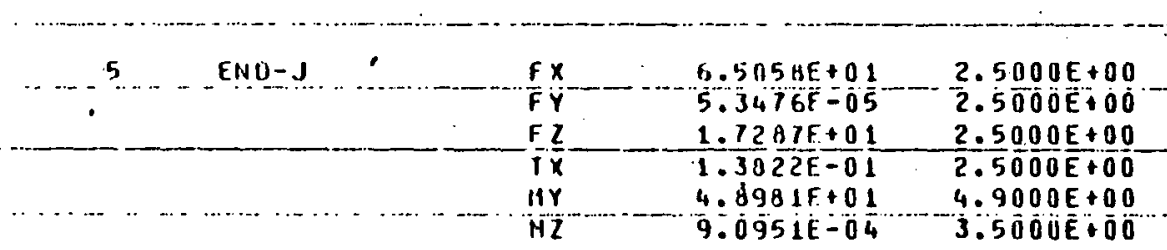

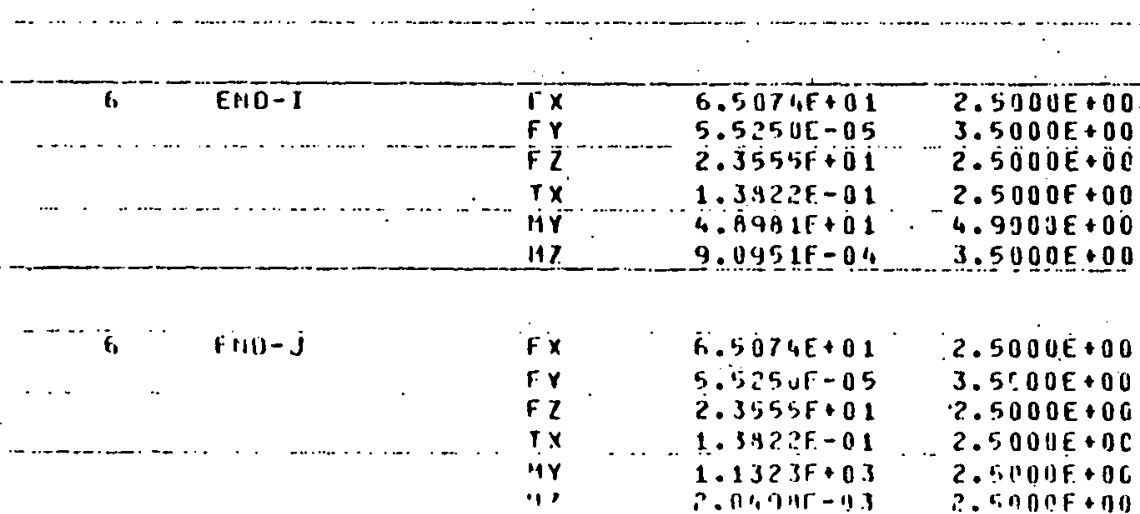




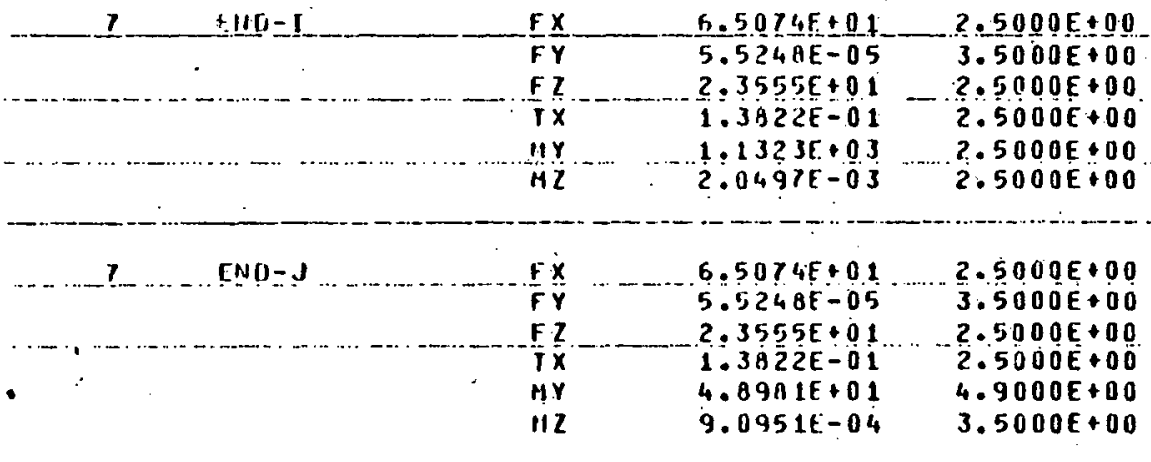

END

\begin{tabular}{|c|c|c|}
\hline $\begin{array}{l}F_{y} \\
F_{y}\end{array}$ & $6.5058 \mathrm{E}+01$ & 2.5000 \\
\hline $\begin{array}{l}I^{2} \\
I_{x}\end{array}$ & $1.7287 E+01$ & 2.5000 \\
\hline$M \vec{v}$ & 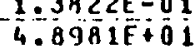 & $\begin{array}{l}2.5000 t \\
4.9000 \mathrm{E}\end{array}$ \\
\hline $\mathrm{HZ}$ & $9.0951 E-04$ & $3.5000 \mathrm{E}$ \\
\hline
\end{tabular}

ENB-J

$\begin{array}{ll}F X & 6.5058 E+01 \\ F Y & 5.3474 E-05 \\ F Z & 1.7287 E+01 \\ T X & 1.3822 E-01 \\ \text { HY } & 8.0603 E+02 \\ M Z & 3.2207 E-03\end{array}$

$2.5000 E+00$

$2.5000 E+0$

$2.5000 E+00$

$2.5000 E+00$
$2.5000 E+00$

2.5000E+00

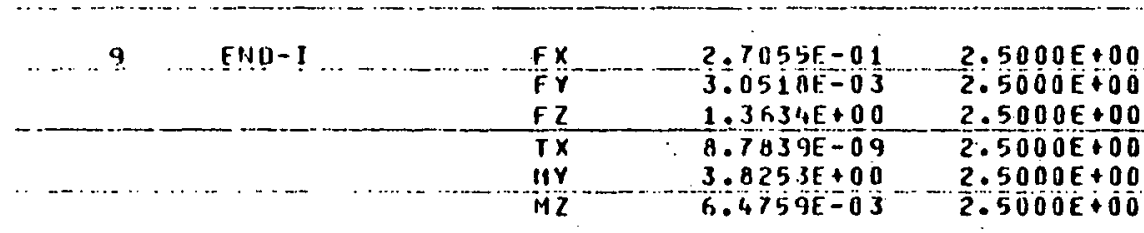

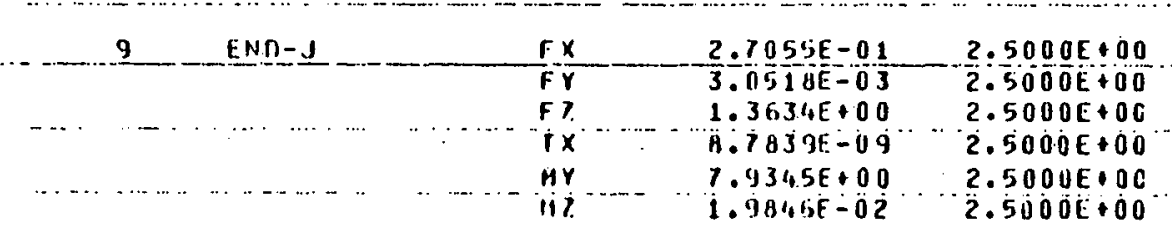

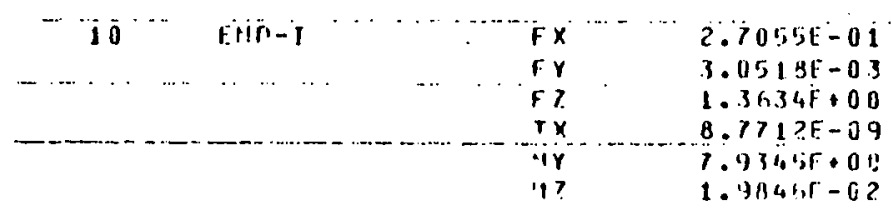

$2.50000+00$

2. $500 J E+00$

$2.5 C O U E+O Q$

$2.500 \mathrm{OEOB}$

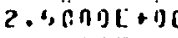

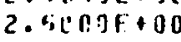

0




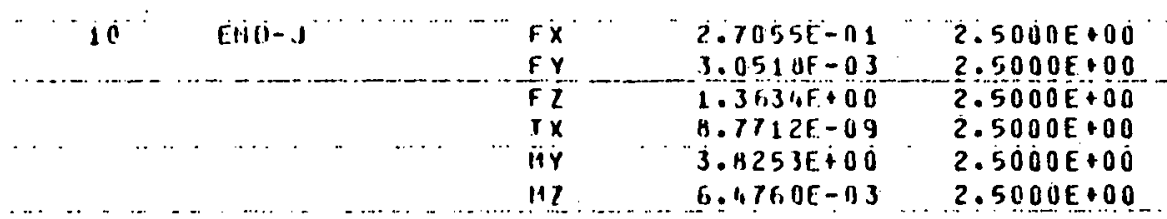

\begin{tabular}{|c|c|c|c|c|}
\hline$\ldots 11$. & $F H(1-I$ & $\begin{array}{l}F x \\
F y\end{array}$ & $\begin{array}{l}h .505 B E+01 \\
5.3476 E-05\end{array}$ & $\begin{array}{l}2.5000 E+00 \\
2.5000 E+00\end{array}$ \\
\hline & & $\begin{array}{l}F Z \\
T X \\
M Y\end{array}$ & $\begin{array}{l}1.7287 E+01 \\
1.3822 E-01 \\
0.8603 E+C 2\end{array}$ & $\begin{array}{l}2.5000 E+00 \\
2.5000 E+00 \\
2.5000 E+00\end{array}$ \\
\hline & & $M Z$ & $3.220 \mathrm{AF}=03$ & $2.5000 E+00$ \\
\hline
\end{tabular}

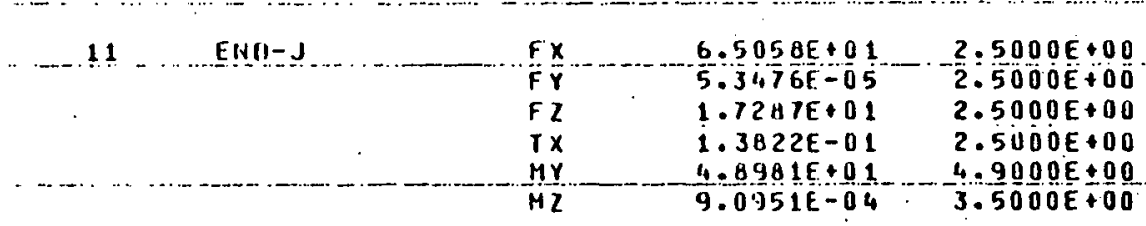

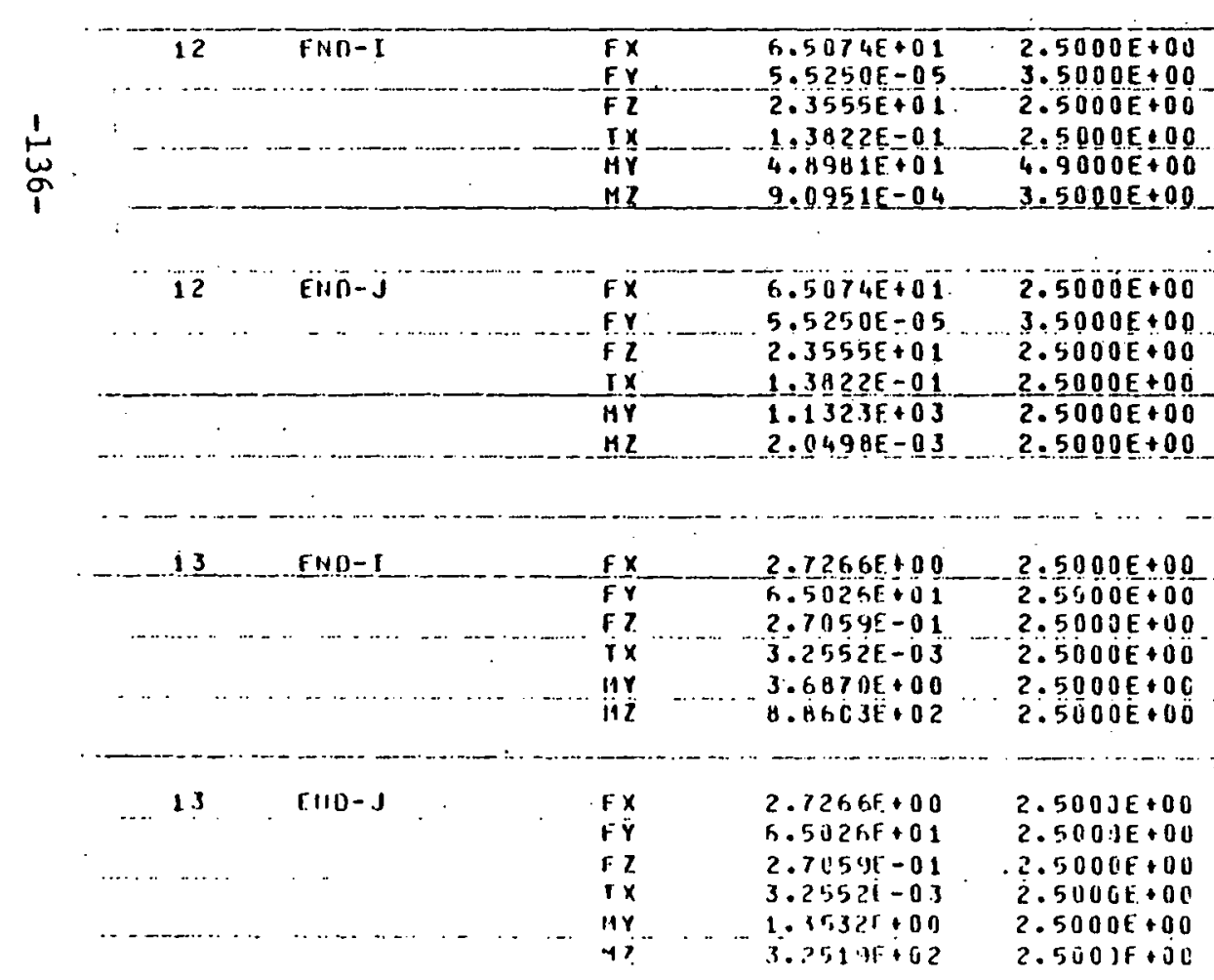




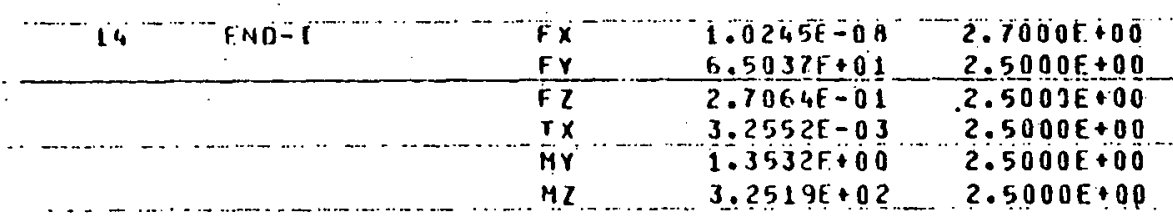

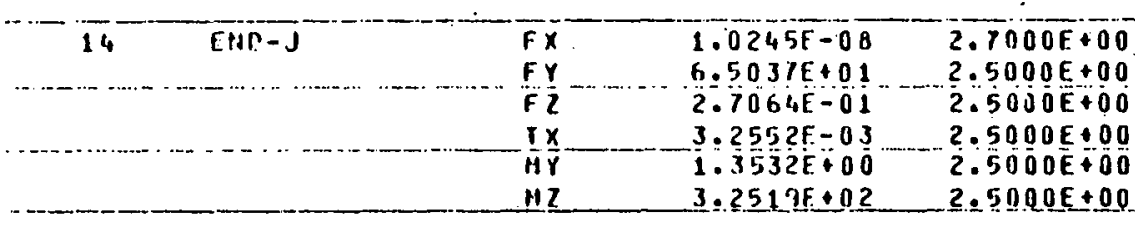

\begin{tabular}{|c|c|c|c|}
\hline 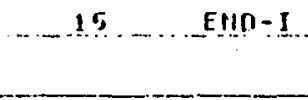 & $\begin{array}{l}F x \\
F \bar{y} \\
F \underline{z}\end{array}$ & $\begin{array}{l}2.7266 E+00 \\
6.5026 E+01 \\
2.7059 F=01\end{array}$ & $\begin{array}{l}2.5000 E+00 \\
2.5000 E+00 \\
2.5000 E+00\end{array}$ \\
\hline & $\begin{array}{l}T x \\
\text { Hy } \\
\text { iis }\end{array}$ & $\begin{array}{l}3.2552 E-03 \\
1.3532 E+00 \\
3.2519 E+02\end{array}$ & $\begin{array}{l}2.5000 E+00 \\
2.5000 E+00 \\
2.5000 E+00\end{array}$ \\
\hline
\end{tabular}

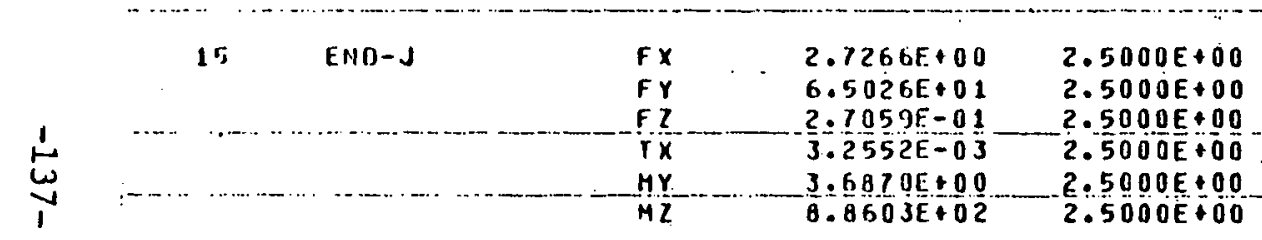

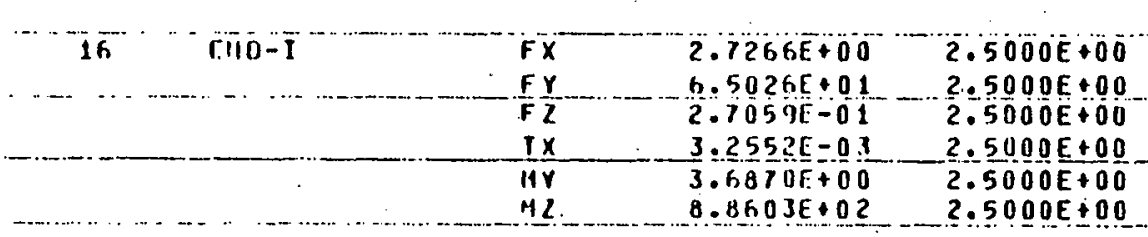

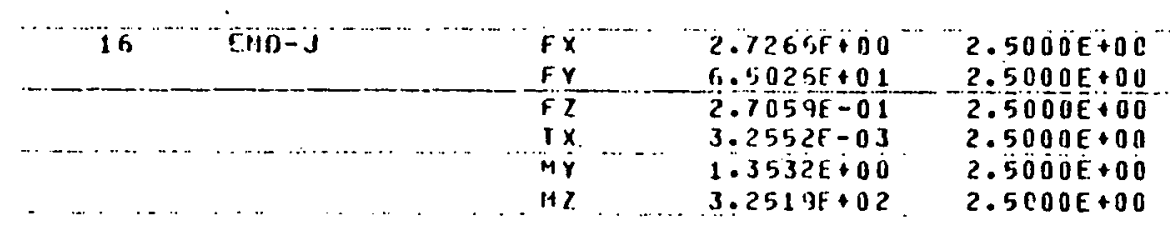

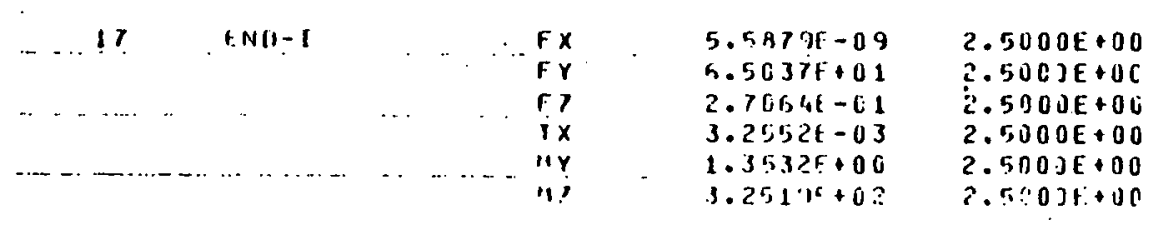




\begin{tabular}{|c|c|c|c|c|}
\hline$\ldots+17$ & .... & $\begin{array}{r}F \mathbf{X} \\
\boldsymbol{F} \mathbf{Y} \\
F \underline{F}\end{array}$ & $\begin{array}{l}5.5 A 7 U E-09 \\
6.503 j E=01 \\
2.7064 F-01\end{array}$ & $\begin{array}{l}2.5000 E+00 \\
2.5000 E+00 \\
2.5000 E+00\end{array}$ \\
\hline & & $\begin{array}{l}7 \bar{x} \\
M y \\
i y\end{array}$ & $\begin{array}{r}3.2552 E-03 \\
1.3532 E+00\end{array}$ & $\begin{array}{l}2.5000 E+00 \\
2.5000 E+00\end{array}$ \\
\hline
\end{tabular}

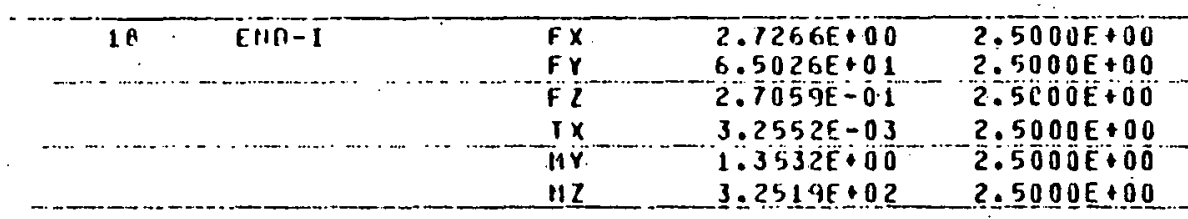

\begin{tabular}{|c|c|c|c|}
\hline " is $\cdots$ E(iii)-J & $\begin{array}{l}F \bar{x} \\
F y \\
F \bar{z} \\
i x\end{array}$ & $\begin{array}{l}2.7266 E+00 \\
6.5026 E+01 \\
2.7059 E-01 \\
3.2552 E-03\end{array}$ & $\begin{array}{l}2.5000 E+00 \\
2.5000 E+00 \\
2.5000 E+00 \\
2.5000 E+00\end{array}$ \\
\hline & $\begin{array}{l}M Y \\
M 2\end{array}$ & $\begin{array}{l}3.6870 E+00 \\
B .8603 E+02\end{array}$ & $\begin{array}{l}2.5000 E+00 \\
2.5000 E+00\end{array}$ \\
\hline
\end{tabular}




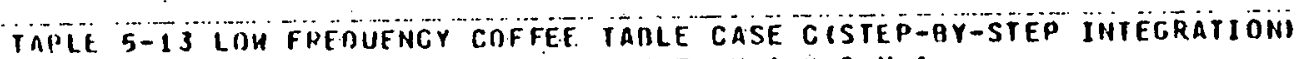

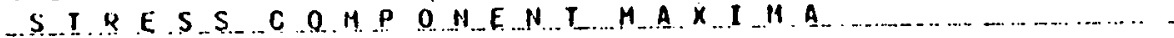

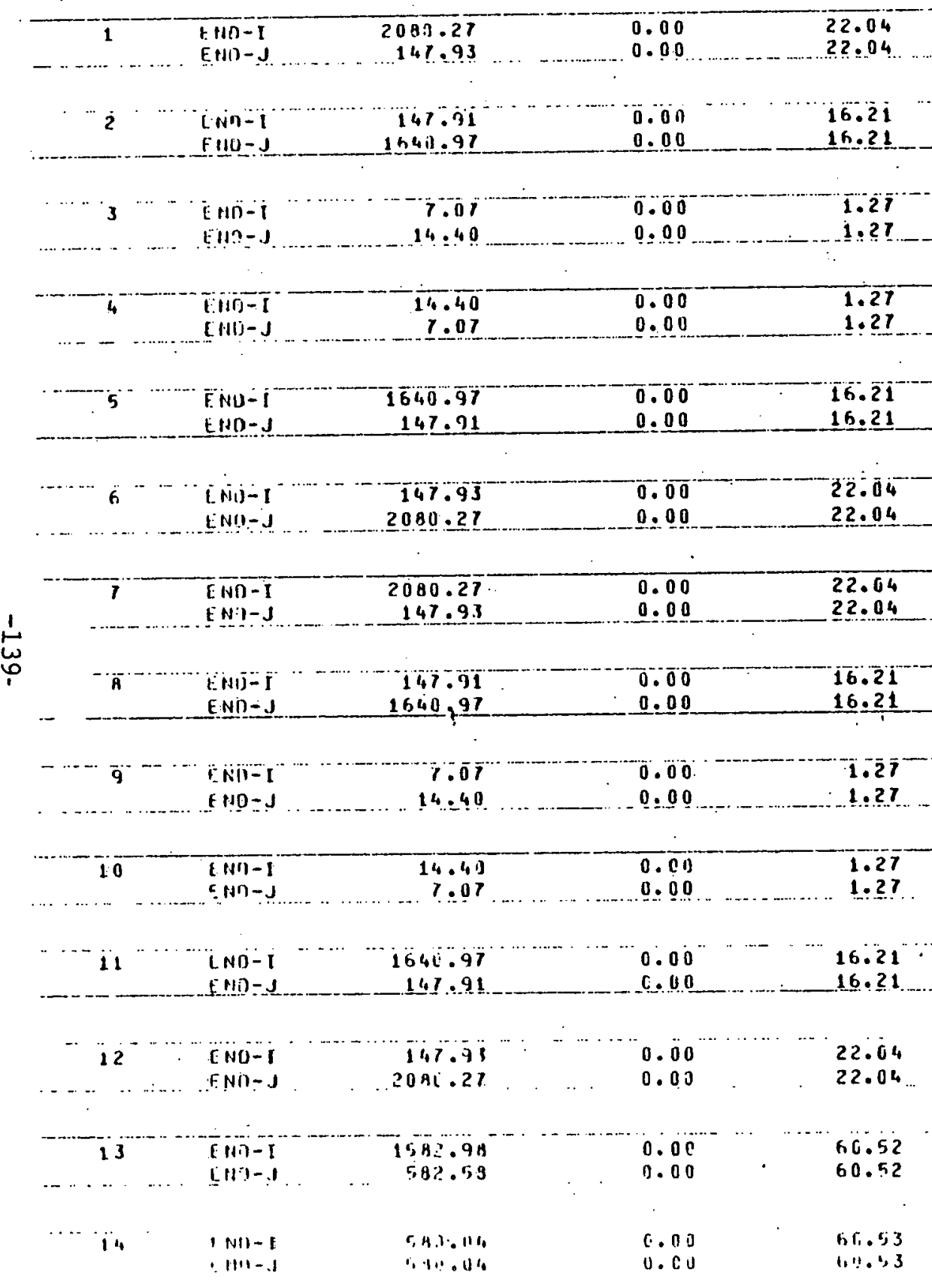




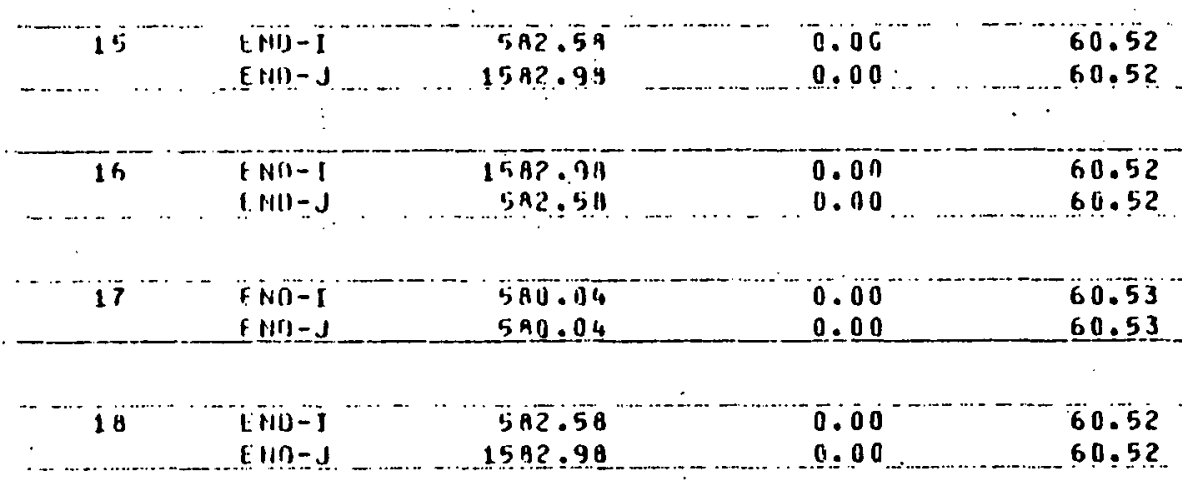

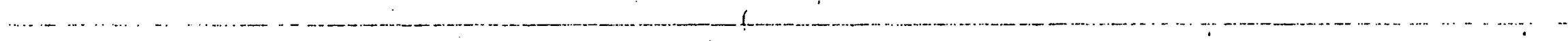

(n)

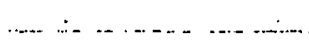




\section{References}

1. Reich, M., Esztergar, E.P., spence, J., Boyle, J., Chang, T.Y., "Elastic and Inelastic Methods of Piping Systems Analvsis: A Preliminary Review Brookhaven National Laboratory, Upton, New York 11973, BNL-19768, February, 1975

2. Pressure Vessel and Pioing - 1972 Computer programs Verification, Ed. by Tuba, I.S. and Wright, W.B., The American Society of Mechanical Engineers, New York, (1972).

3. "EPIPE - An Elastic Piping Program for static and Dynamic Analyses" - A BNL report in publication.

4. Bathe, K.J., Wilson, E.I., and Peterson, F.E., "SA. IV A Structural Analysis Program for Static and Dynamic Responses of Linear Systems," Report No. EERC 73-11, University of California, Berkeley, California, (1973).

5. ASME Boiler and pressure Vessel Code, Section III-1971, Nuclear Power Plant Components, American Society of Mechanical Engineers, New York, (July 1, 1971).

6. Martin, H.C.. Introduction to Matrix Methods of Structural Analvsis, MCGraw-Hill, NerN York, (1966).

7. Poley, S., "Mesh Analys is of Piping. Systems," IBM New York Scientific Center Technical Report No. 320-2939, (March 1968).

8. Rodabough, E.C. and George; H.H., "Effect of Internal Pressure on Flexibility and Stress Intensification Factors of Curved pipe or Welding Elbows," Trans. ASME, vol. 79, (1957).

9. Cal Tech Report.

10. Clough,.W.R., "Earthquake Response of Structures," Earthquake Engineering, Ed. by Wiegel, R.L., PrenticeHall, New Jersey, (1970).

11.: Spence, J., "Private Communication," (November, 1975). 
12. Hovgaard, W., "Stresses in Three Dimensional Pipe Bends;" Trans. ASME, Vol. 57, FSP-57-12, P401-476, (1935):

13. Hovgaard, N.,"Further Studies of Three Dimensional Pipe Bends," Trans. ASME, FSP-50-13, Vol. 59, p. 647650, (1937).

14. PIPDYN II - A Computer Program for the Complete Analysis and Evaluation of Piping Systems by Zudans, Z., et. al.; Applied Mechanics Lab., The Franklin Institute Research Lab., Philadelphia, PA.

15. Crede, C.E., "Shock and Vibration Concepts in Engineering Design," Englewood Cliffs, NJ, Prentice-Hall (1965).

16. Swanson, J., "A Problem on Dynamic Analysis of Three Dimensional Structure," Pressure Vessel and Piping, 1972 Computer Program Verification, Ed. by Tuba, IoS. and Wright, W.B., American Society of Mechanical Engineers, New York, (1972).

17. Tuba, I. S. and. Wright, W. B., "Pressure Vessel and Piping, 1972 Computer Programs Verification," ASME Pressure Vessel and Piping Division, New York (1972).

18. Bezler, P., Gardner, D, and Hartzman, M., "A Benchmark Solution for a Hypothetical Reactor System," BNL-NUREG 23645 , July, 1977. 